\title{
Population balance modeling of influenza $A$ virus replication in MDCK cells during vaccine production
}

\section{Dissertation}

zur Erlangung des akademischen Grades

\section{Doktoringenieur \\ (Dr.-Ing.)}

von Dipl.-Ing. Thomas Müller

geb. am 01.12.1979 in Magdeburg

genehmigt durch die Fakultät für Elektrotechnik und Informationstechnik der Otto-von-Guericke-Universität Magdeburg

Gutachter:

Prof. Dr.-Ing. Achim Kienle

Prof. Dr.-Ing. Udo Reichl

Prof. Dr.-Ing. Heiko Briesen

Promotionskolloquium am 17.02.2015 


\section{Acknowledgments}

This thesis is the result of work within the Research Center for Dynamic Systems. The funding of this project by the state of Saxony-Anhalt is hereby gratefully acknowledged.

Firstly, I would like to express my gratitude to Prof. Dr.-Ing. Achim Kienle for his professional support and patience as a doctoral adviser. Furthermore, I address thanks to the entire staff of the Chair for Automation/Modeling for providing a pleasant working environment, in particular, to Robert Dürr for his support on parameter estimation and for being a good office mate.

I am thankful to Prof. Dr.-Ing. Udo Reichl and his Bioprocess Engineering group at the Max Planck Institute for Dynamics of Complex Technical Systems in Magdeburg for sharing their expertise and for granting access to much appreciated experimental data. In this regard, special thanks are due to Josef Schulze-Horsel and Britta Peschel.

Finally, I would like to express my heartfelt gratitude to my family and, most of all, my partner Peggy for their support and understanding before, during, and after the creation of this thesis. 


\section{Abstract}

Influenza is a highly contagious disease of the respiratory tract, which not only occurs regularly in seasonal waves, but can also assume pandemic proportions in irregular intervals. By affecting several million people each year influenza causes serious healthcare and economic problems worldwide. The related pathogenic agent is influenza virus, which is classified into types A, B, and C. Among these three types influenza A virus is the most notable human influenza virus due to its remarkable antigenic variability and wide range of possible hosts resulting in the ever new challenge of controlling not only the seasonal influenza waves but also potential epidemics or even pandemics.

Vaccination is recognized as the most effective method to prevent influenza infection. Hence, the efficient adaptation of the vaccine production is of vital importance in order to successfully take on this challenge. In consequence, cell culture-based methods become increasingly relevant for influenza vaccine production as they are less time consuming and far more flexible than the traditionally performed production process in embryonated chicken eggs. In combination with extensive experimental work mathematical modeling helps to promote this development by contributing significantly to the understanding and optimization of the process.

The work at hand is concerned with the development of a deterministic population balance model of the influenza A virus infection of adherent Madin-Darby canine kidney (MDCK) cells during vaccine production. One of the prominent features of such a multicellular system is the remarkable heterogeneity of the involved cell population, which can be measured systematically with the help of flow cytometry and has a decisive influence on the dynamics of the process. Therefore, a distributed modeling approach is chosen that pays special attention to the rigorous consideration of flow cytometric data in order to account for the present cell-to-cell variability. It is the first time, that this is done for the influenza vaccine production.

The presentation of the model is subdivided into several evolutionary steps that are associated with certain key concepts that eventually lead to the finally presented model formulation. These are the degree of fluorescence as an internal coordinate, the explicit consideration of cells in the latent phase, and the assumption of a second phase of replication. These key concepts and the underlying assumptions are motivated by the available experimental data. The resulting implications and limitations are discussed.

Simulation results are directly compared with distributed flow cytometric data of the temporal change of the intracellular amount of viral nucleoprotein, which reveals characteristic dynamic phenomena, like transient multimodality and reversal of propagation direction. The presented model allows for a consistent explanation of the dynamic behav- 
ior of heterogeneous cell populations during the production of different influenza A virus strains and, thereby, helps to give an insight into the underlying biological processes. 


\section{Zusammenfassung}

Bei der Influenza oder Virusgrippe handelt es sich um eine hochansteckende Erkrankung der oberen und unteren Atemwege, die regelmäßig in saisonalen Infektionswellen auftritt, welche sich in unregelmäßigen Abständen auch zu Pandemien ausweiten können. Jedes Jahr infizieren sich auf diese Weise mehrere Millionen Menschen mit Influenza, was weltweit starke Belastungen des Gesundheitssystems hervorruft und schwerwiegende ökonomische Einbußen zur Folge hat. Der Erreger ist das Influenzavirus, von welchem die Typen A, B und C unterschieden werden. Von diesen drei Typen kann das InfluenzaA-Virus aufgrund seiner herausragenden Variabilität in Bezug auf seine antigenen Eigenschaften und der breiten Palette an möglichen Wirten als die wohl bemerkenswerteste Spezies angesehen werden. Genau diese Eigenschaften sind es nämlich, welche die Bekämpfung sowohl der saisonalen Grippewellen als auch möglicher Epidemien oder sogar Pandemien zur immer wieder neuen Herausforderung machen.

Da die Impfung als effektivste Methode zur Verhinderung einer Infektion mit Influenza angesehen wird, nimmt die effiziente Anpassung der Impfstoffproduktion als Mittel zur erfolgreichen Bewältigung dieser Aufgabe einen hohen Stellenwert ein. Dabei gewinnen zellbasierte Impfstoffproduktionsverfahren immer mehr an Bedeutung, weil sie gegenüber der herkömmlichen Herstellungsmethode in bebrüteten Hühnereiern mit weniger zeitlichem Aufwand und dazu auch weitaus flexibler durchführbar sind. In Verbindung mit ausgiebiger experimenteller Arbeit helfen mathematische Modelle dabei, diese Entwicklung voranzutreiben, indem sie wesentlich zum Verständnis sowie zur Optimierung des Prozesses beitragen.

Im Mittelpunkt der vorliegenden Arbeit steht die Entwicklung eines deterministischen, populationsdynamischen Modells für die Influenza-A-Virusinfektion von adhärenten MDCK-Zellen während der Impfstoffproduktion. Eine der herausstechendsten Eigenschaften des betrachteten multizellulären Systems ist die ausgeprägte Heterogenität innerhalb der beteiligten Zellpopulation. Diese lässt sich mittels Durchflusszytometrie systematisch ermitteln und hat entscheidenden Einfluss auf die Dynamik des gesamten Prozesses. Um die vorhandene Zellvariabilität entsprechend berücksichtigen zu können, wurde daher ein Modellansatz mit eigenschaftsverteilten Zustandsgrößen verfolgt. Dieser Ansatz stellt ein Novum auf dem Gebiet der Impfstoffproduktion dar.

Das Modell wird anhand der wesentlichen Entwicklungsschritte vorgestellt, welche jeweils mit einem bestimmten Schlüsselkonzept in Verbindung stehen und somit einen Teil zur letztendlich präsentierten Modellformulierung beitragen. Bei diesen Schlüsselkonzepten handelt es sich um den Fluoreszenzgrad als Eigenschaftskoordinate, die direkte Berücksichtigung von Zellen in der Latenzphase und die Annahme einer zweiten Replikationsphase. Die Auswahl dieser Konzepte und die zugrundeliegenden Modellan- 
nahmen fußen direkt auf den zur Verfügung stehenden experimentellen Daten. Die sich daraus ergebenden Schlussfolgerungen und Einschränkungen werden entsprechend diskutiert.

Simulationsergebnisse werden direkt mit verteilten Daten aus der Durchflusszytometrie verglichen. Diese Daten umfassen den zeitlichen Verlauf der intrazellulären Menge viralen Nukleoproteins, anhand dessen sich charakteristische, dynamische Phänomene wie transiente Multimodalität oder die Umkehr der Ausbreitungsrichtung feststellen lassen. Das vorgestellte Modell erlaubt eine konsistente Erklärung dieses dynamischen Verhaltens heterogener Zellpopulationen bei der Produktion verschiedener Influenza-AVirusstämme und trägt daher dazu bei, das Verständnis der zugrundeliegenden, biologischen Prozesse zu vergrößern. 


\section{Contents}

1 Introduction 1

1.1 Motivation . . . . . . . . . . . . . . . . . . 1

1.2 State of the art . . . . . . . . . . . . . . . . 2

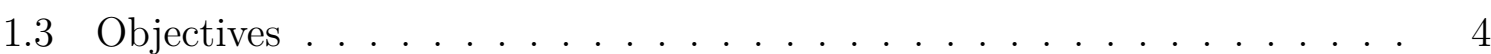

1.4 Outline . . . . . . . . . . . . . . . . . . 6

2 Lumped modeling according to Möhler et al. [2005] 9

2.1 Process description and model assumptions . . . . . . . . . . . . . . . . . 9

2.2 Model equations . . . . . . . . . . . . . . . . . . . . . . . . . 10

2.3 Numerical solution . . . . . . . . . . . . . . . . . . . . . . . 12

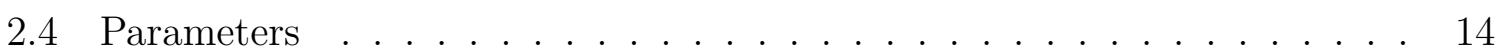

2.5 Simulation results . . . . . . . . . . . . . . . 15

3 Distributed modeling of equine influenza A virus replication 17

3.1 Preprocessing of flow cytometric data . . . . . . . . . . . . . . . . . . . 19

3.2 Process description and model assumptions . . . . . . . . . . . . . . . 21

3.3 Model equations . . . . . . . . . . . . . . . . . . . . . . . . 22

3.4 Numerical solution . . . . . . . . . . . . . . . . . . . . . 25

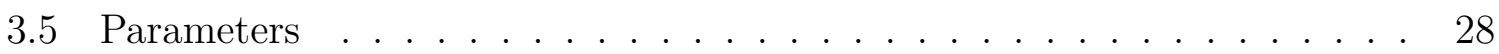

3.6 Simulation results . . . . . . . . . . . . . . . . . . . . 32

4 Implementation of the backshift 43

4.1 Model equations . . . . . . . . . . . . . . . . . . . 43

4.2 Parameters . . . . . . . . . . . . . . . . . . . 44

4.3 Simulation results . . . . . . . . . . . . . . . . . . 46

5 Distributed modeling of human influenza A virus replication 51

5.1 Expansion of the experimental database . . . . . . . . . . . . . 51

5.2 Influence of apoptosis . . . . . . . . . . . . . . . . . . . 52

5.3 Process description and model assumptions . . . . . . . . . . . . . . . 54

5.4 Model equations . . . . . . . . . . . . . . . . . . . . 56

5.5 Numerical solution . . . . . . . . . . . . . . . . . . . 59

5.6 Parameter estimation . . . . . . . . . . . . . . . . . 61

5.7 Simulation results . . . . . . . . . . . . . . . . . . 64

5.8 Special case A/H1N1-NIBSC . . . . . . . . . . . . . . . . . 78

6 Conclusion $\quad 85$

6.1 Summary . . . . . . . . . . . . . . . . . . . . . 85

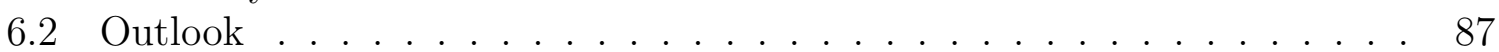

6.2.1 Continuous alteration of the replication dynamics . . . . . . . . 87

6.2.2 Distinction between the eclipse phase and the latent phase . . . . 89 
6.2.3 Multiple infections . . . . . . . . . . . . . . . . . . . . . 90

6.2.4 Defective interfering particles . . . . . . . . . . . 92

6.2.5 Consideration of an immune response . . . . . . . . . . . . 95

$\begin{array}{ll}\text { Appendix } & 97\end{array}$

A Determination of the limit of the scaling factor in Eq. (3.7) 97

B Derivation of Eq. (3.12) 99

C Discretization via finite-volume method (FVM) 101

C.1 Discretization of Eq. $(2.5) \ldots \ldots \ldots \ldots 10 \ldots$

C.2 Discretization of Eq. (3.18) . . . . . . . . . . . . . . . . 102

C.3 Discretization of Eq. (3.21) . . . . . . . . . . . . . . . 104

C.4 Discretization of Eq. (5.10) . . . . . . . . . . . . . 106

C.5 Discretization of Eq. (5.4) . . . . . . . . . . . . . . 107

$\begin{array}{ll}\text { Nomenclature } & 109\end{array}$

$\begin{array}{ll}\text { List of Tables } & 115\end{array}$

$\begin{array}{lr}\text { List of Figures } & 117\end{array}$

$\begin{array}{ll}\text { References } & 119\end{array}$ 


\section{Introduction}

\subsection{Motivation}

Influenza is a highly contagious disease of the respiratory tract, which affects several million people each year worldwide. It regularly occurs in seasonal waves, which peak in the winter months. Additionally, worldwide pandemics occur in infrequent intervals. Although it is not restricted to a certain group of people, seasonal influenza particularly affects infants, elderly people, and people with a weakened immune system or with chronic diseases. As a result, influenza not only causes serious healthcare and economic problems, but it is also responsible for the death of 250000 to 500000 people each year [World Health Organization, 2009].

The related pathogenic agent is the influenza virus - an RNA virus which belongs to the family of Orthomyxoviridae. The virus spreads by airborne infection, hand contact with contaminated surfaces and subsequent hand to mouth or hand to nose contact. It is classified into types $\mathrm{A}, \mathrm{B}$, and $\mathrm{C}$ of which the latter usually causes only light illness and is much less prevalent than the other two types [Katagiri et al., 1983; Taubenberger and Morens, 2008]. Therefore, type $\mathrm{C}$ is not included in the seasonal influenza vaccine. Influenza B virus infections occur periodically and can cause severe illness [Belshe, 2010; Domachowske et al., 2013]. Consequently, it is part of the seasonal vaccine, though, due to its limited host range [Osterhaus et al., 2000] and comparably small antigenic variation [Lindstrom et al., 1999; Nobusawa and Sato, 2006], type B is not able to cause pandemics. The most notable human influenza pathogen is influenza A virus. It is characterized by a very high genetic and antigenic variability making it capable of regularly outflanking the human immune defense resulting in severe annual epidemics. Furthermore, influenza $\mathrm{A}$ is able to cause worldwide pandemics at irregular intervals when an antigenically new virus makes contact with an immunologically unprepared human population [Hay et al., 2001]. Influenza A virus is subdivided into several subtypes according to the structure of the two glycoproteins hemagglutinin (HA) and neuraminidase (NA), which are part of the virus envelope and play an important role in the attachment to (HA) and the release from (NA) the host cells. Type $\mathrm{A}$ is able to infect a variety of mammals and birds with particularly water birds forming the natural reservoir - all known influenza A subtypes have been validated in water bird populations [Webster et al., 1992]. The most common subtypes circulating in the human population are A/H1N1 and A/H3N2 [Robert Koch Institut, 2011].

The high genetic variability of influenza A virus results in ever changing demands in the handling of not only the seasonal influenza waves but also potential epidemics or even pandemics. As vaccination is the most effective method to prevent influenza infection, 
the efficient adaptation of the vaccine production is of vital importance. Based on the evaluation of virological and epidemiological data from all around the world, the World Health Organization (WHO) recommends the seasonal influenza vaccine composition of the coming season. This is done every February and September for the northern and southern hemisphere, respectively. After the development, testing and standardization of virus candidates, vaccine manufacturers are provided with the respective virus strains and production starts.

Traditionally, influenza vaccine production takes place in embryonated chicken eggs, which is a well-established and relatively cheap process, which also has certain disadvantages - e.g., the capacity to produce a sufficient amount of chicken eggs is limited, the process is time consuming and rather inflexible when changing demands have to be met, with certain virus strains only low yields can be reached, the compliance with biological safety standards is challenging, in case of an avian influenza pandemic the whole production is in danger, and allergic reactions against egg products have to be considered [Nichols and LeDuc, 2009; Schulze-Horsel, 2011]. These disadvantages can be overcome by cell culture-based methods, which are already widely-used for other viruses [Nielsen, 2000] and become increasingly relevant for influenza vaccines as well. To promote this development and to improve the production process not only sophisticated cell culturebased technologies and downstream processing methods are required. In combination with extensive experimental work mathematical modeling contributes significantly to the understanding and optimization of the process.

\subsection{State of the art}

The modeling of multicellular systems can be a difficult task. This holds particularly true for the modeling of vaccine production processes, which are characterized by the complex interplay of a heterogeneous cell population with the respective virus. Conventional mathematical models describing cell and virus dynamics during vaccine production consider the respective populations of infected and uninfected cells to behave homogeneously, leading to a system of ordinary differential equations [Möhler et al., 2005; Enden et al., 2005; Schulze-Horsel et al., 2009]. By this approach all cells of a certain population are lumped together and considered to behave exactly the same. Thus, the heterogeneity of the cell population and the variability of the cell physiology, which can have a decisive influence on the overall process, are neglected.

With the help of flow cytometry it is possible to systematically collect data of various properties that characterize heterogeneous cell populations [Nichols et al., 1993; Srienc, 1999; Schulze-Horsel et al., 2008]. In Fig. 1 an example of flow cytometric measurements is given for the infection of an adherent cell culture with influenza A virus. In traditional 


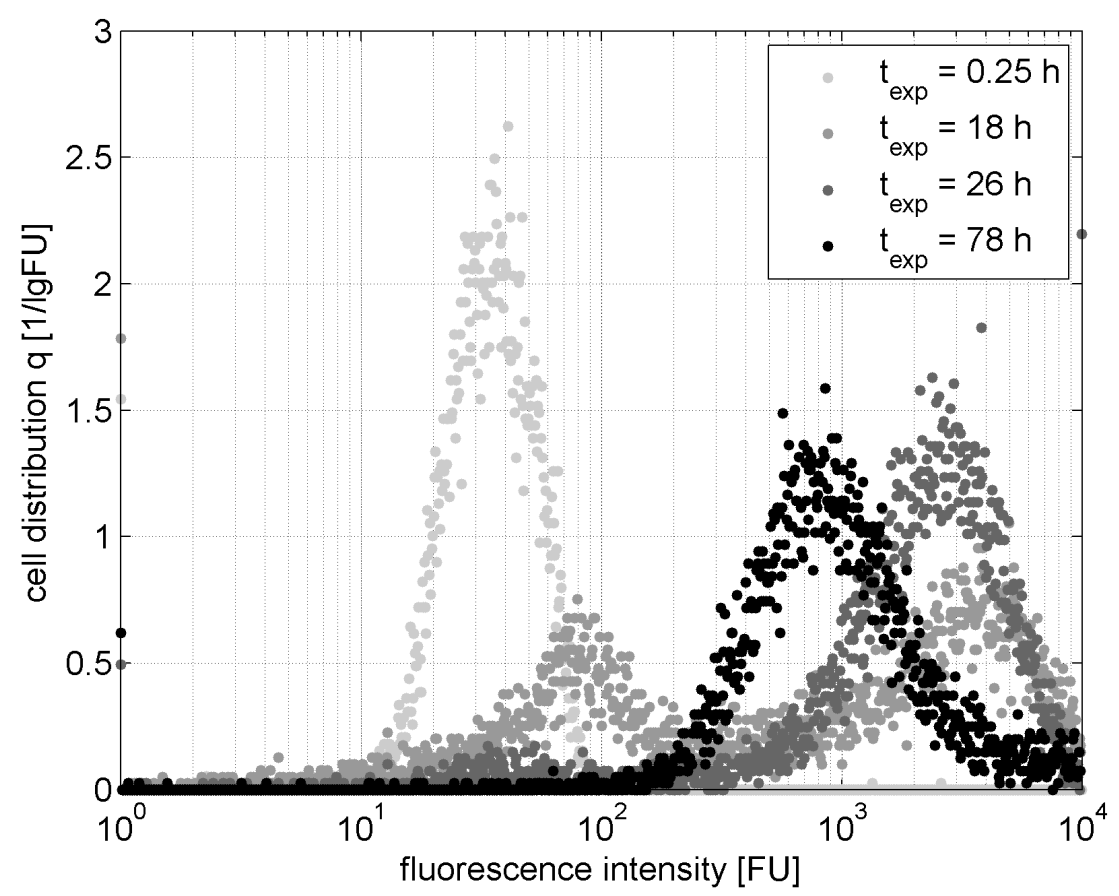

Figure 1: Example of flow cytometric data that reveal characteristic dynamic phenomena of the cell population during the infection of adherent Madin-Darby canine kidney (MDCK) cells with human influenza A/Wisconsin/67/2005 (H3N2) virus [Schulze-Horsel, 2011]. The distribution of the host cell population over the fluorescence intensity is depicted for several times post infection. The fluorescence intensity is proportional to the amount of flouresceinconjugated antibodies against the viral target protein, in this case nucleoprotein (NP). The necessary preprocessing of the experimental data is explained in Section 3.1.

lumped model approaches basically only the mean values of these measurements are of interest. In contrast to this, a distributed approach is applied here, which also accounts for cell-to-cell variability represented by the data.

To benefit from the available information from flow cytometry, which allows the consideration of the cell-to-cell variance, a suitable modeling framework has to be adopted. In principle, one can choose between stochastic and deterministic approaches. In a stochastic setting it is possible to consider a larger number of internal state variables. Thus, a stochastic framework is of advantage when a structured model approach is pursued in which many intracellular components have to be considered explicitly for individual cells. On the other hand, by using a deterministic approach one benefits from advanced methods of model analysis, parameter identification as well as process design and control.

In order to account for the heterogeneity in multicellular systems, population balance 
approaches offer a suitable deterministic framework [Ramkrishna, 2000]. Population balance equations, which originate from the Boltzmann equation, are used to describe the dynamic change of the distribution of particles or, in a more general sense, entities within a predefined state space [Ramkrishna and Mahoney, 2002]. The wider application of population balance approaches to a diverse array of problems started in the early 1960s. Fredrickson et al. published a major article on the statistics and dynamics of microbial growth in which they employed population balance equations [Fredrickson et al., 1967]. From there on, the description of biological cell cultures by means of population balance equations was further promoted by this scientific work group, e.g. Ramkrishna [1979], Fredrickson [1991], Fredrickson and Mantzaris [2002], Fredrickson [2003].

In the early 1990s several groups were concerned with the mathematical modeling of the baculovirus replication in insect cell cultures, e.g. de Gooijer et al. [1992], Licari and Bailey [1992], or Power et al. [1992]. In particular, Nielsen and Power utilized so called age-structured models in which the performance of the host cells depends on the time since their infection [Power et al., 1994; Power and Nielsen, 1996; Nielsen, 2000]. More recently, Haseltine and Rawlings also relied on an age-structured approach to study the virus replication in cell cultures in several generic settings [Haseltine et al., 2005, 2008]. Therein, events on the intracellular as well as the extracellular level are considered, assuming that cells of the same age since infection show equal performance. Although these approaches were a significant contribution to the field of virus dynamics, no particular application was considered and a comparison to experimental data is lacking.

After previous approaches by Sidorenko et al. [2008a,b], which utilized a stochastic approach, focus in the current work is on deterministic population balance modeling. A direct comparison with distributed experimental data obtained by flow cytometry (see Fig. 1) is provided. Note, that most important results of this thesis have been published in advance in Müller et al. [2008, 2011, 2013] and Dürr et al. [2012].

\subsection{Objectives}

This work is concerned with the development of a deterministic population balance model of the influenza A virus infection of adherent Madin-Darby canine kidney (MDCK) cells during vaccine production. Special attention is paid to the rigorous consideration of flow cytometric data in order to simulate the dynamic behavior of heterogeneous cell populations. It is the first time, that this is done for the influenza vaccine production.

The experimental setting is described in detail by Schulze-Horsel et al. [2009]. The considered process takes place in a small-scale bioreactor (1.2l working volume). Therein adherent MDCK cells are grown on microcarriers until, eventually, a confluent mono- 


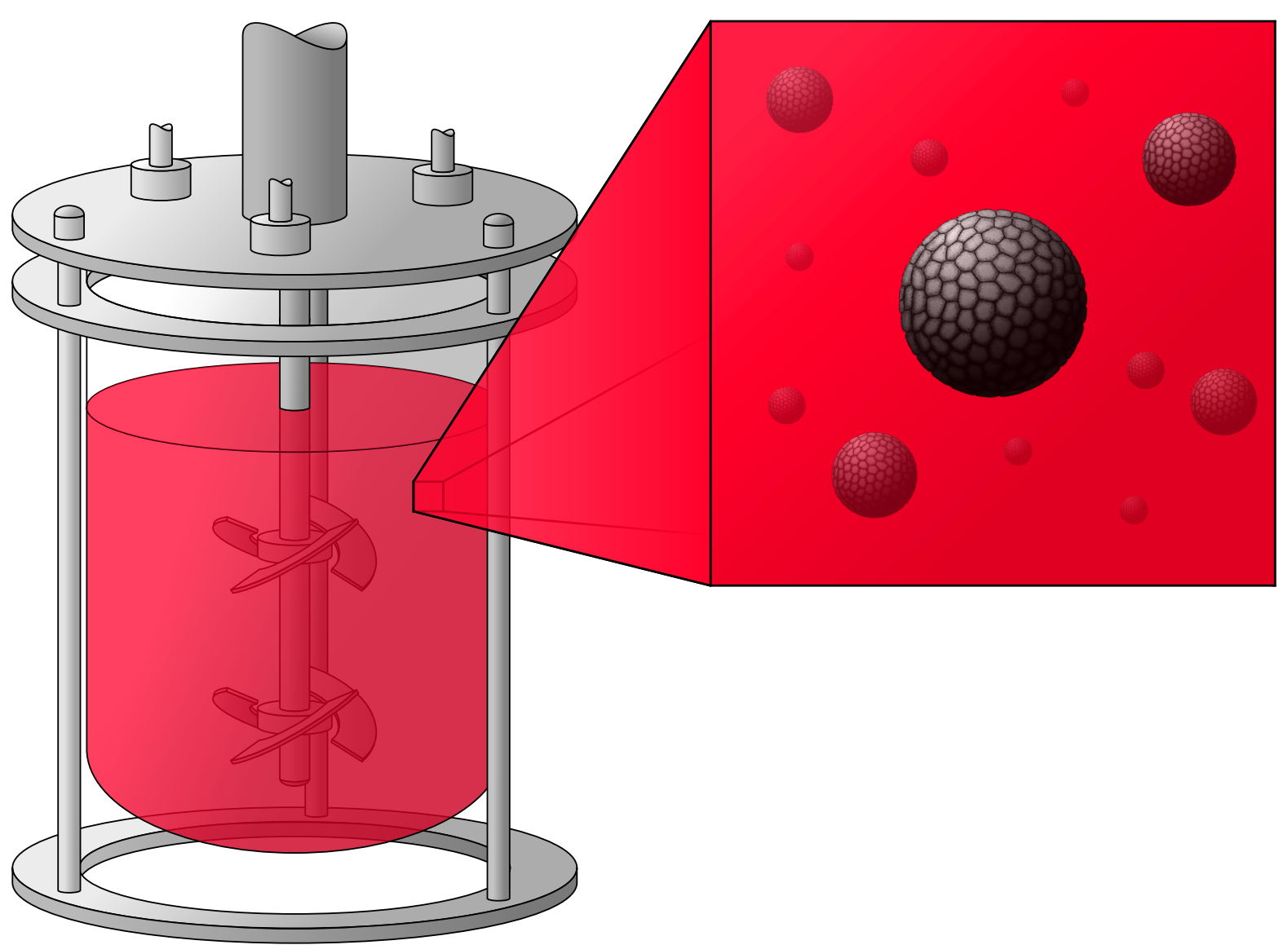

Figure 2: Illustration of a bioreactor with a magnified detail depicting confluently overgrown microcarriers.

layers is reached [Genzel et al., 2004] (see Fig. 2). After the removal of spent medium and a subsequent washing step, fresh medium is added and the cell culture is inoculated with the virus seed containing infectious/active and uninfectious/inactive virus particles. Active virions attach to uninfected cells and infect them subsequently. Although inactive virus particles can also bind to the cell membrane and incorporation is possible, successful infection and replication do not take place. For simplicity, other biologically active virus particles, like defective interfering particles (DIPs), are neglected and inactive virus particles are assumed to simply accumulate in the supernatant without further interaction with the host cell population.

Newly infected cells undergo the eclipse phase [Flint et al., 2009], during which neither virus replication nor release can be detected. With regard to the presented model formulations the eclipse phase lumps together events happening before viral protein synthesis, e.g. entry of the virus into the host cell, uncoating of nucleic acid, various transport processes and RNA transcription. After completion of the eclipse phase infected cells 
start to synthesize viral protein. The change of the intracellular amount of viral protein, in the present case nucleoprotein (NP), is measured via flow cytometry and can be used to characterize the progress of infection [Schulze-Horsel et al., 2009; Müller et al., 2013]. This allows the differentiation of the host cell population and gives rise to distributed modeling approaches. Experimental results reveal characteristic dynamic phenomena on the distribution level like transient multimodality and reversal of propagation direction. In Fig. 1 (see page 3) a typical course of events is presented on the basis of actual experimental data of four selected time points. In a first phase (between the infection and $t_{\exp }=18 \mathrm{~h}$ ) the unimodal initial distribution transforms into a transient bimodal distribution. Afterwards, the two distinct peaks unite again at high fluorescence intensities $\left(t_{\exp }=26 \mathrm{~h}\right)$. By comparison of the distributions at $t_{\exp }=26 \mathrm{~h}$ and $t_{\exp }=78 \mathrm{~h}$ the back-propagation to lower fluorescence intensities becomes apparent. The main objective of this work is the consistent explanation of these characteristic phenomena in order to provide insight into the underlying biological processes.

\subsection{Outline}

The thesis is organized as follows. After the introduction (Section 1) the basic model of Möhler et al. [2005] is presented in Section 2 as this lumped approach lay the foundation for the following distributed models. In addition, this section constitutes a steppingstone to the understanding of the following model formulations.

Taking off from there, in Section 3 a distributed modeling approach is introduced that directly utilizes flow cytometric results of equine influenza A infections of MDCK cells. Therefore, a degree of fluorescence is established as an internal coordinate, which is related to the intracellular amount of viral nucleoprotein (NP). It is shown how raw flow cytometric data have to be processed in order to be comparable to simulation results and how the resulting model allows to draw conclusions about the course of events in the experiments. Strengths and weaknesses of the proposed model are discussed.

As the lacking ability to reproduce the reversal of the propagation direction of the cell distribution, the so called backshift, is regarded the main drawback of the model, in Section 4 a possible mechanistic model formulation is presented that resolves this deficit by proposing a two-phase replication process. Though, due to sparse experimental data at the time the model was developed no physiological reason could be determined for the transition from the first to the second phase.

The situation changed when more dense experimental data were available for human influenza A replication in adherent MDCK cells. Experimental investigation of the occurrence and the course of apoptosis during influenza vaccine production by Schulze-Horsel 
et al. [2009] and Schulze-Horsel [2011] provided a possible explanation for the backshift. In Section 5 the respectively derived model is presented, discussed and compared with experimental data.

Finally, in Section 6 the main results of this contribution are summed up and evaluated, and additional aspects, which promise to improve the model performance, are presented. 


\section{Lumped modeling according to Möhler et al. [2005]}

From a historical point of view, the model of Möhler et al. [2005] forms the starting point of the models that are presented within this work. For this reason it is introduced here.

In 2005 Möhler et al. presented a deterministic population balance model of equine influenza A virus replication in MDCK microcarrier culture. The model is unstructured and represents a very basic description of the vaccine production process by considering the interactions of three lumped species - uninfected cells, infected cells, and free virus particles. The model was fitted to experimental virus titers measured via hemagglutination assay. In addition, two model formulations were set against each other. These formulations differed in how the time delay between the infection of a cell and the release of the first virus particles was considered. In the first case the lag was considered indirectly by simply shifting the simulation results by a certain amount $\left(t_{\text {shift }}=12 \mathrm{~h}\right)$. In the second case the model consisted of time-delay differential equations that explicitly accounted for the lag period - this second version is described in Section 2.2.

Despite its simple setup the model was able to reproduce the experimental results. Particularly with respect to the maximum virus yield both model formulations performed comparably well.

In the following a short description of the process and the model assumptions are given. For a more detailed presentation the reader is referred to the original publication of Möhler et al. [2005].

\subsection{Process description and model assumptions}

Prior to inoculation, cell growth is completed, i.e. all microcarriers are completely covered by uninfected cells, so that the whole population is contact inhibited. At the time of infection (TOI) virus seed is added with a multiplicity of infection (MOI) of 0.025, i.e. the ratio of infectious virus particles to uninfected cells. Accordingly, free virions are adsorbed by uninfected cells. As not all virus particles are infective only a fraction of these cells actually gets infected. Together with the MOI the infection kinetics determine how many cells are infected by the inoculum. It is assumed that already infected cells are no longer able to adsorb virus particles. Instead, after a certain delay $\tau$ they start to release free virus particles into the medium. This delay is called latent period [Flint et al., 2009] and denotes the time from the successful infection to the release of the first virus particles. During the latent period or latent phase processes like the entry of the virus 
into the host cell, the uncoating of the nucleic acid, viral protein and RNA synthesis as well as virus formation and budding take place. Freshly released virions attach to still uninfected cells and finally infect them. Infected cells keep releasing virus particles until they disintegrate due to virus-induced cell damage or apoptosis. Furthermore, uninfected cells die due to cultivation conditions. While dying, cells are supposed to detach from the microcarrier surface and make room for still uninfected cells, which then are able to grow again. As free virus particles are exposed to unspecific proteases in the medium, they are subject to degradation.

Further important model assumptions: Virus particles are assumed to exclusively attach to uninfected cells. There are no multiple infections. Furthermore, there is no differentiation between cells of the same species. This means, for instance, that all uninfected cells behave the same and all infected cells behave the same, too. Hence, the latent period is considered to have the same length for every cell as well. Uninfectious virions are considered indirectly by using different coefficients for virus attachment and infection. In general, the number of virus particles that attach to uninfected cells should be higher than or at least equal to the number of successfully infected cells. Defective interfering particles (DIPs) are not considered.

\subsection{Model equations}

In order to be in line with further model formulations that are to be presented in this contribution, the nomenclature is slightly changed in comparison to the one used by Möhler et al. [2005]. For the description of the process three state variables are considered: the concentrations of uninfected and infected cells ( $U_{\mathrm{c}}$ and $I_{\mathrm{c}}$, respectively), as well as the concentration of free virus particles $V$. In the beginning all cells are uninfected and the temporal change in concentration is described by

$$
\begin{gathered}
\frac{\mathrm{d} U_{\mathrm{c}}(t)}{\mathrm{d} t}=\mu_{\mathrm{c}}(t) U_{\mathrm{c}}(t)-k_{\mathrm{cdf}} U_{\mathrm{c}}(t)-k_{\mathrm{vi}} U_{\mathrm{c}}(t) V(t) \\
\text { with } \quad \mu_{\mathrm{c}}(t)=\mu_{\mathrm{c}, \max } \frac{C_{\max }-\left(U_{\mathrm{c}}(t)+I_{\mathrm{c}}(t)\right)}{C_{\max }}
\end{gathered}
$$

The concentration of uninfected cells $U_{\mathrm{c}}$ is increased by cell growth with the cell growth coefficient $\mu_{\mathrm{c}}$. Culture conditions are maintained in a way, so that factors like insufficient substrate availability or limiting metabolites are excluded [Genzel et al., 2004]. Only the finite microcarrier surface restricts cell growth. Hence, $\mu_{\mathrm{c}}$ is defined by the maximum concentration of cells supported by all microcarriers $\left(C_{\max }\right)$ and the maximum growth coefficient $\mu_{\mathrm{c}, \max }$. Thus, $\mu_{\mathrm{c}}$ approaches $\mu_{\mathrm{c}, \max }$ when the overall number of cells $\left(U_{\mathrm{c}}+I_{\mathrm{c}}\right)$ 


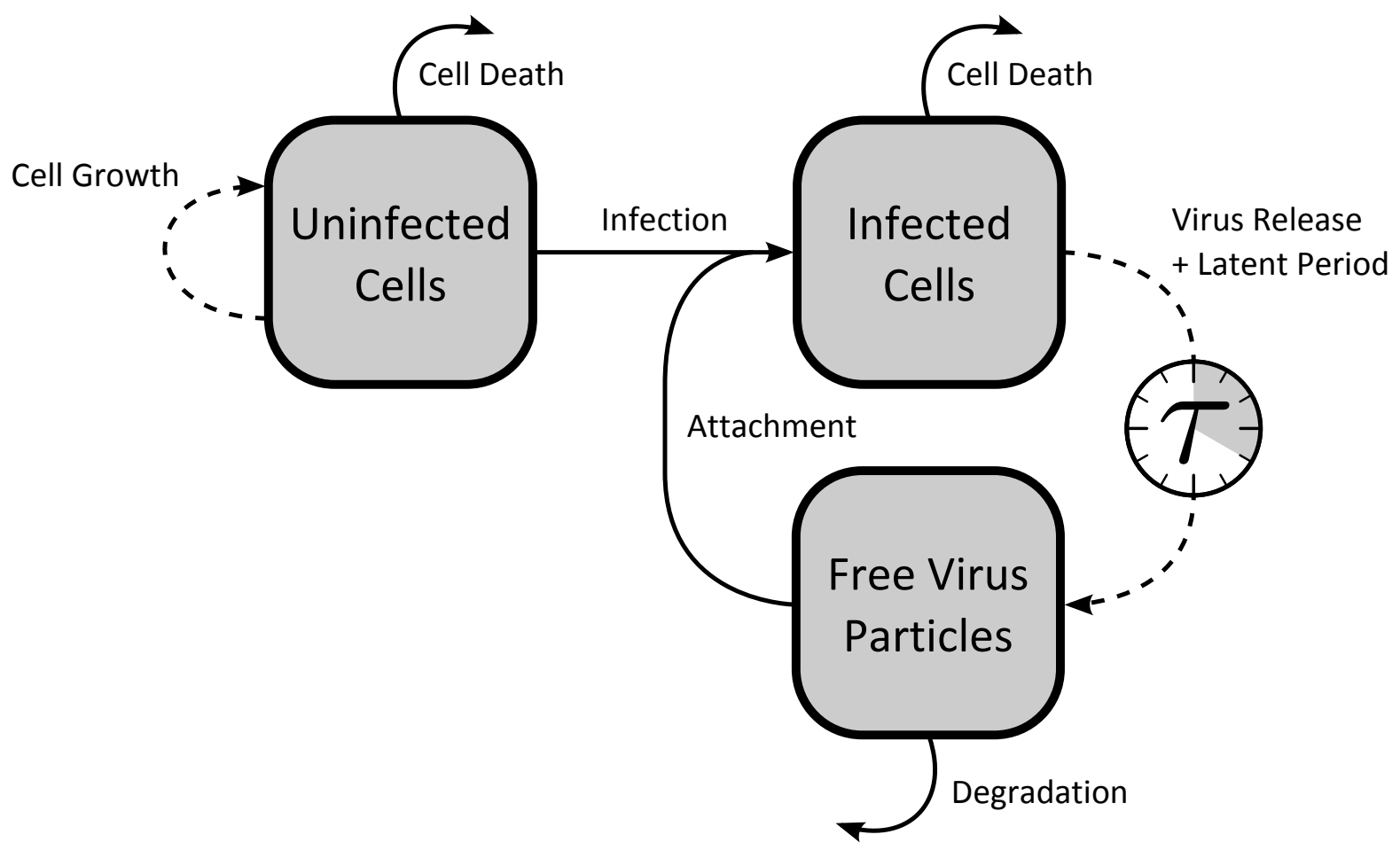

Figure 3: Scheme of the model of Möhler et al. [2005]. Solid arrows indicate the consumption of the species the arrow originates from. If the arrow points at another species, the latter is produced simultaneously. Dashed arrows also symbolize production which depends on the availability of the species the arrow originates from. But in this case the required species is not consumed during the process.

tends to zero. On the other hand, cell growth stops when the overall concentration of cells reaches its maximum $C_{\max }$. Uninfected cells die with the cell death coefficient due to cultivation conditions $k_{\mathrm{cdf}}$. Infection is proportional to the infection coefficient $k_{\mathrm{vi}}$.

Infected cells arise from uninfected cells by successful infection. They eventually die with the cell death coefficient due to viral infection $k_{\text {cdv }}$ :

$$
\frac{\mathrm{d} I_{\mathrm{c}}(t)}{\mathrm{d} t}=k_{\mathrm{vi}} U_{\mathrm{c}}(t) V(t)-k_{\mathrm{cdv}} I_{\mathrm{c}}(t)
$$

After passing through the latent period of length $\tau$ infected cell start to produce and release virus particles with the release rate $k_{\text {rel }}$. Free virions degrade with the degradation coefficient $k_{\mathrm{vd}}$ and attach to uninfected cells with the attachment coefficient $k_{\mathrm{va}}$ :

$$
\frac{\mathrm{d} V(t)}{\mathrm{d} t}=k_{\mathrm{rel}} I_{\mathrm{c}}(t-\tau)-k_{\mathrm{vd}} V(t)-k_{\mathrm{va}} U_{\mathrm{c}}(t) V(t)
$$

A scheme of the described processes is presented in Figure 3. 


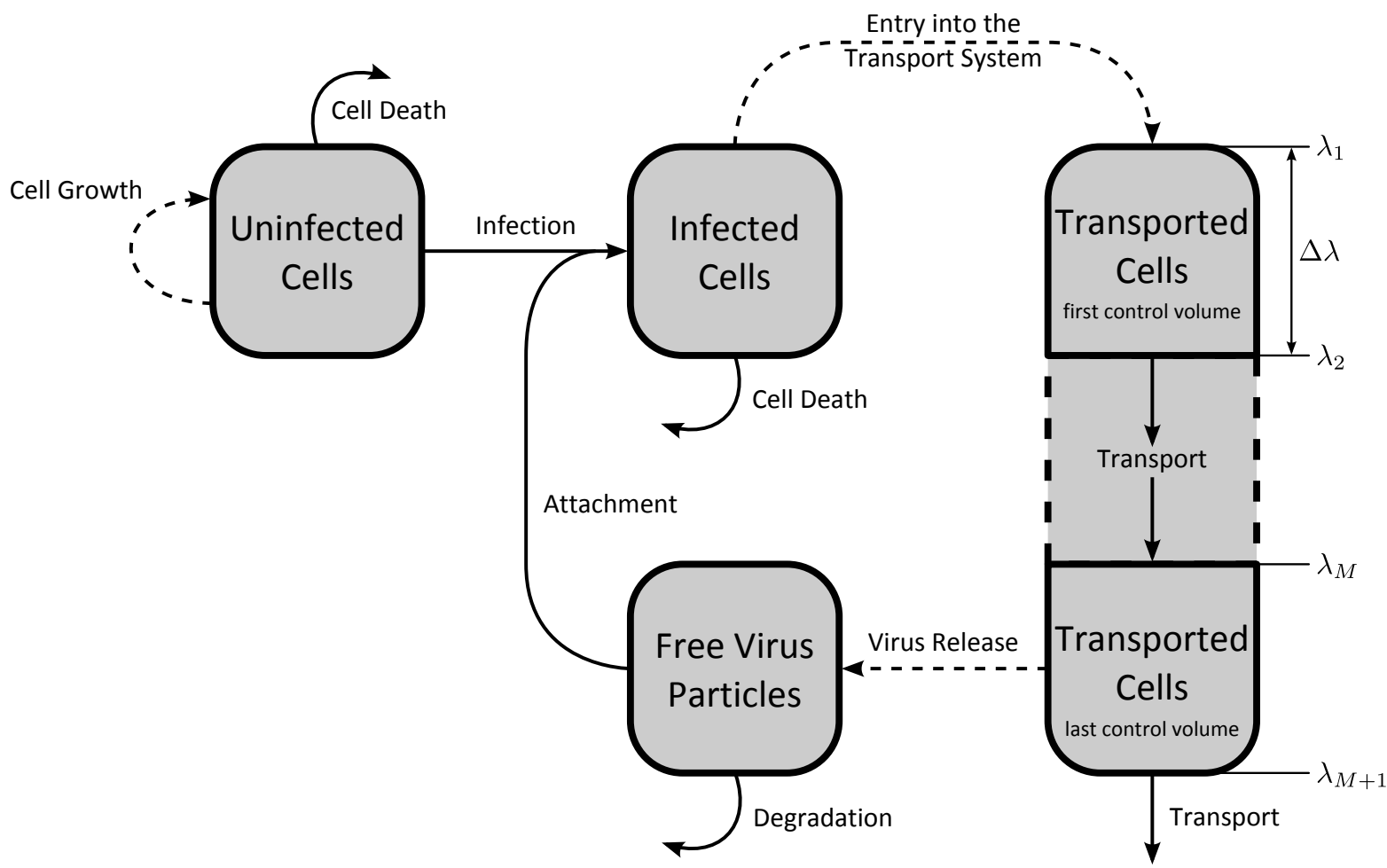

Figure 4: Scheme of the model of Möhler et al. [2005] with the latent period being approximated by a discretized transport system. The transported cells originate from infected cells, travel through the transport system along the transport coordinate $\lambda$, and start to release virus particles when they eventually reach the end of the transport system.

\subsection{Numerical solution}

For the numerical solution the latent period is represented by a transport system of constant length. Therefore, a new state variable $T_{\mathrm{c}}$ is introduced, which represents the number density along the nondimensional transport coordinate $\lambda \in \mathbb{R},[0,1]$ :

$$
\frac{\partial T_{\mathrm{c}}(t, \lambda)}{\partial t}=-\frac{1}{\tau} \frac{\partial T_{\mathrm{c}}(t, \lambda)}{\partial \lambda}
$$

Cells in the transport system travel with a constant transport velocity $1 / \tau$. The entry of infected cells into the transport system is specified by the boundary condition:

$$
T_{\mathrm{c}}(t, \lambda=0)=I_{\mathrm{c}}(t) .
$$

When the transported cells reach the end of the transport system at time $t$ their concentration is equal to the concentration of infected cells at time $t-\tau$ :

$$
T_{\mathrm{c}}(t, \lambda=1)=I_{\mathrm{c}}(t-\tau)
$$




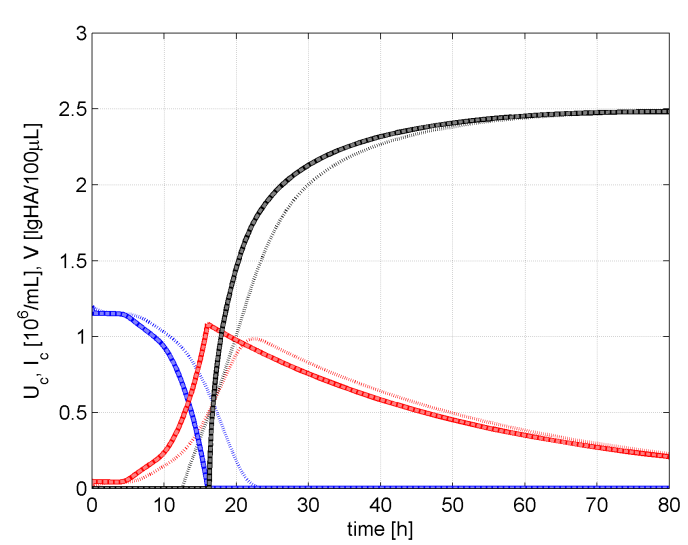

a) Simultaneous change of $k_{\mathrm{va}}$ and $k_{\mathrm{vi}}$ - dashed (dotted) lines represent simulation results when both coefficients are multiplicated by $10^{-3}\left(10^{-6}\right)$.

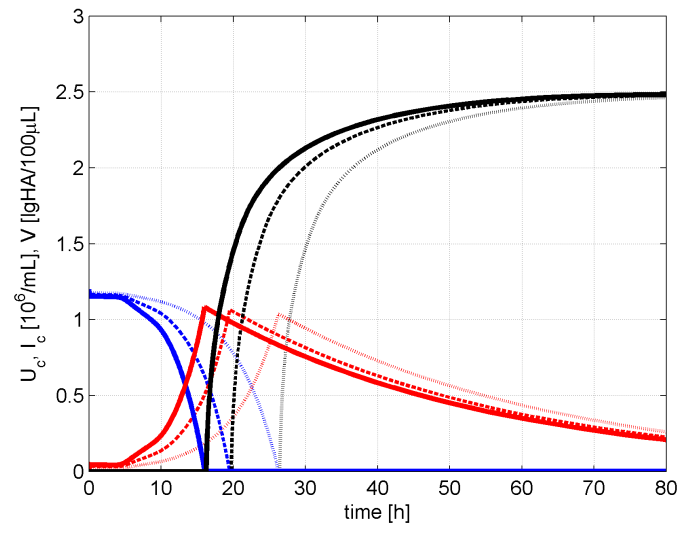

b) Exclusive change of $k_{\mathrm{vi}}$ - dashed (dotted) lines represent simulation results when $k_{\mathrm{vi}}$ is multiplicated by $0.75(0.5)$.

Figure 5: Influence of the attachment coefficient $\left(k_{\mathrm{va}}\right)$ and the infection coefficient $\left(k_{\mathrm{vi}}\right)$ on the dynamics of the model of Möhler et al. [2005] - solid lines represent the concentrations of uninfected cells (blue), infected cells (red), and virus particles (black) when the parameter set of Tab. $\mathrm{I}$ is applied.

Equation (2.5) is discretized with the help of the finite-volume method (FVM) using an equidistant discretization scheme with $M=64$ control volumes, which turned out to be a sound compromise between accuracy and effort (see Appendix C.1, page 101):

$$
\begin{gathered}
\frac{\mathrm{d} T_{\mathrm{c}, i}(t)}{\mathrm{d} t}=-\frac{1}{\Delta \lambda \tau}\left(T_{\mathrm{c}, i}(t)-T_{\mathrm{c}, i-1}(t)\right) \\
\text { with } \quad T_{\mathrm{c}, 0}(t)=I_{\mathrm{c}}(t) .
\end{gathered}
$$

Thus, the virus balance equation (2.4) can be substituted by

$$
\frac{\mathrm{d} V(t)}{\mathrm{d} t}=k_{\mathrm{rel}} T_{\mathrm{c}, M}(t)-k_{\mathrm{vd}} V(t)-k_{\mathrm{va}} U_{\mathrm{c}}(t) V(t)
$$

A scheme of the resulting model structure is presented in Figure 4.

In order to numerically solve this and all further models the dynamic simulator DivA [Mangold et al., 2000] was used. Of all the available solvers implemented in DivA DDASAC [Caracotsios and Stewart, 1985] turned out to be the most suitable. Data processing and visualization as well as additional computations were performed with MatLAB [2012].

Although the model structure is simple, the proposed kinetics can generate numerical problems. The infection and attachment terms can yield extreme values causing the 
concentrations of uninfected cells or virus particles to overshoot below zero. This can be reduced by decreasing the solver step size and the allowed numerical error. In order to prevent the occurrence of negative values appropriate constraints to the state variables have been applied to the balance equations.

Note, that a reasonable reduction of the parameters $k_{\mathrm{va}}$ and $k_{\mathrm{vi}}$ can reduce the problem without affecting the overall system dynamics as long as the ratio between both parameters is preserved and the change is kept within certain limits. If, for instance, both $\left(k_{\mathrm{va}}\right.$ and $k_{\mathrm{vi}}$ ) are multiplied by $10^{-3}$ no significant change of the system dynamics can be observed, whereas an exclusive reduction of $k_{\mathrm{vi}}$ by one quarter results in a significant change that is comparable to a simultaneous multiplication by $10^{-6}$ (see Fig. 5).

\subsection{Parameters}

In order to demonstrate the validity of the model, the parameters published by Möhler et al. [2005, Tab. I, p. 50] are adopted. In Table I the parameter set and initial conditions are summarized. The cell death coefficient of uninfected cells $\left(k_{\mathrm{cdf}}\right)$, the virus degradation coefficient $\left(k_{\mathrm{vd}}\right)$, and the maximum growth coefficient $\left(\mu_{\mathrm{c}, \max }\right)$ were determined in independent experiments. All other parameters were fitted to experimental data taken from the production of equine influenza A/Newmarket/1/93 (H3N8) in MDCK microcarrier culture in a bioreactor with 41 working volume at MOI $=0.025$ [Möhler et al., 2005].

\begin{tabular}{rcl}
\hline Parameter & Value & Unit \\
\hline$C_{\text {max }}$ & $1.2 \cdot 10^{6}$ & $\mathrm{ml}^{-1}$ \\
$k_{\mathrm{cdf}}$ & 0.001 & $\mathrm{~h}^{-1}$ \\
$k_{\mathrm{cdv}}$ & $25.7 \cdot 10^{-3}$ & $\mathrm{~h}^{-1}$ \\
$k_{\mathrm{rel}}$ & 482 & $\mathrm{~h}^{-1}$ \\
$k_{\mathrm{va}}$ & 0.8 & $\mathrm{ml} / \mathrm{h}$ \\
$k_{\mathrm{vd}}$ & 0.009 & $\mathrm{~h}^{-1}$ \\
$k_{\mathrm{vi}}$ & $1.4 \cdot 10^{-3}$ & $\mathrm{ml} / \mathrm{h}$ \\
$\mu_{\mathrm{c}, \max }$ & 0.03 & $\mathrm{~h}^{-1}$ \\
$\tau$ & 4.5 & $\mathrm{~h}$ \\
\hline$I_{\mathrm{c}, 0}$ & 0 & $\mathrm{ml}^{-1}$ \\
$U_{\mathrm{c}, 0}$ & $1.2 \cdot 10^{6}$ & $\mathrm{ml}^{-1}$ \\
$V_{0}$ & $26 \cdot 10^{6}$ & $\mathrm{ml}^{-1}$ \\
\hline
\end{tabular}

Table I: Parameter set and initial conditions for the model of Möhler et al. [2005] 
As an initial condition all cells are uninfected with an initial concentration of $U_{\mathrm{c}, 0}=$ $1.2 \cdot 10^{6} \mathrm{ml}^{-1}$. The virus seed is added at TOI resulting in an initial virus concentration of $V_{0}=26 \cdot 10^{6} \mathrm{ml}^{-1}$.

\subsection{Simulation results}

In Figure 6 the resulting dynamics for the adopted parameter set (Tab. I) are shown. The virus particles of the inoculum all attach to uninfected cells and the virus concentration instantly drops to zero (see Fig. 6b). Due to a relatively low MOI of 0.025 only a small fraction of the uninfected cells is infected and constitutes the first generation of virus producing cells. Though, before the release of new virus particles can begin the freshly infected cells have to pass through their latent phase. Hence, apart from a slight decrease caused by cell death, the concentrations of uninfected and infected cells stay relatively constant for about $4 \mathrm{~h}$.

Eventually, the first generation of infected cells starts to release the first virus particles at about 3.5 hours post infection (h p.i.). The virus concentration increases until a plateau is reached at $6 \mathrm{hp}$.i. As the number of producing cells is rather low, the virus release is balanced out by virus attachment to uninfected cells, which results in a nearly linear decrease of the number of uninfected cells between about 5 to $9 \mathrm{~h}$ p.i. The number of infected cells increases accordingly. At about $8 \mathrm{~h}$ p.i. the first infected cells out of this second generation start to release virus. From this point on virus release accelerates as more and more infected cells drop out of the latent phase. The number of infected cells equally increases with accelerated tempo until finally all cells are infected at $16 \mathrm{~h}$ p.i. With no uninfected cells left, the number of infected cells declines due to cell death. The increase of the virus yield slows down accordingly, but is maintained, as for the duration of the simulation there are enough productive cells left to counter virus degradation. Interestingly, the virus concentration does not rise above the detection limit of the hemagglutination assay $\left(4.06 \cdot 10^{7}\right.$ virusparticles/ml [Möhler et al., 2005]) until the number of infected cells is already declining. 


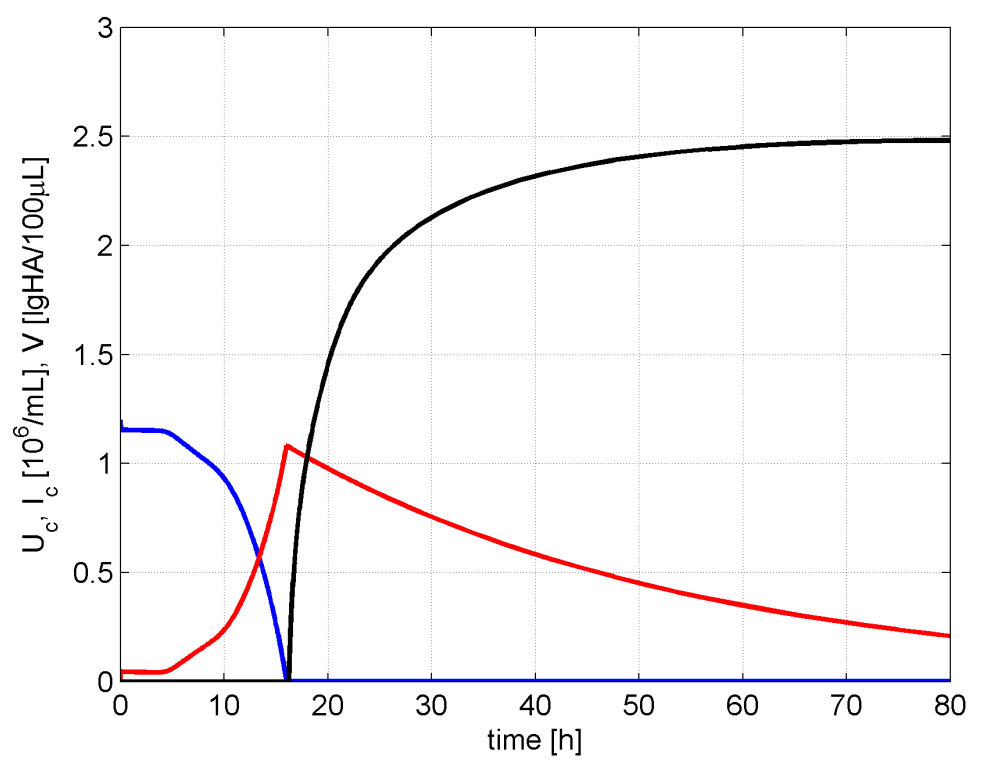

a) Temporal progression of the concentrations of uninfected cells (blue), infected cells (red) and virus particles (black). The virus yield is given in $\frac{\lg \mathrm{HA}}{100 \mu \mathrm{l}}$.

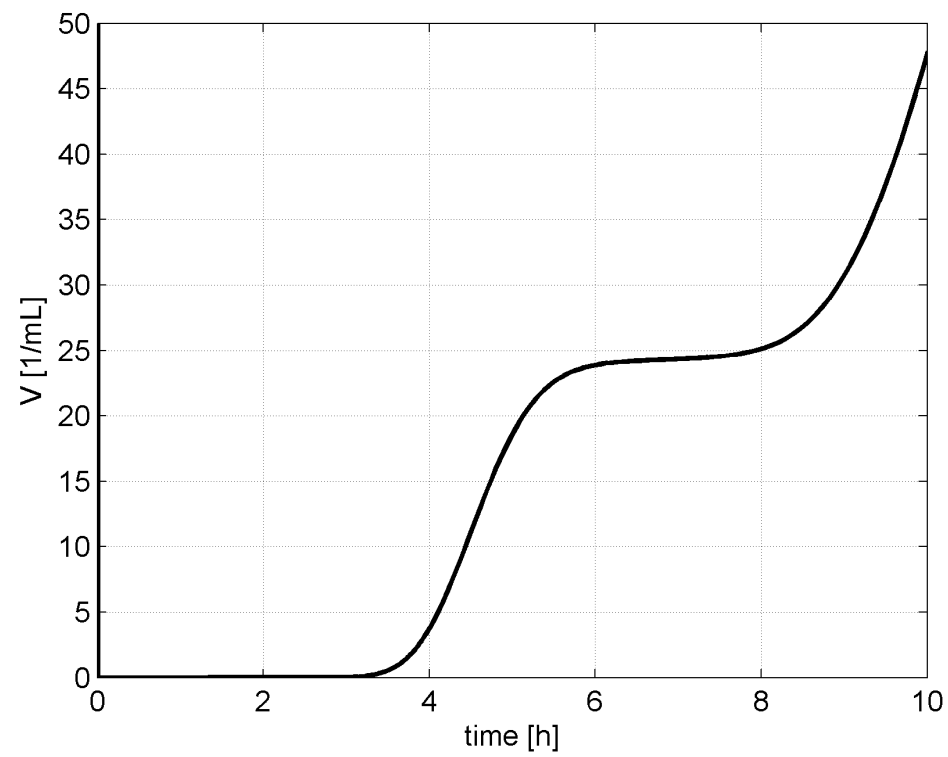

b) Early progression of the virus concentration given in particles $/ \mathrm{ml}$.

Figure 6: Simulation results of the model of Möhler et al. [2005]. For the applied parameters and initial conditions see Tab. I (page 14). Due to a relatively low MOI of 0.025 several waves of infection occur. This is signified by two distinct bends in the curves of the uninfected and infected cells at about 4 and $9 \mathrm{~h}$ p.i.. Likewise, the early progression of the virus concentration illustrates the influence of the latent period and reveals the discrete waves of infection. 


\section{Distributed modeling of equine influenza A virus replication}

In 2008 Schulze-Horsel et al. published an article about the application of flow cytometry for the monitoring of influenza vaccine production in adherent MDCK cell cultures. Therein, it is shown how flow cytometry can be used to quantify the progress of infection of thousands of individual cells by measuring the intracellular amount of viral protein. Therefore, sampled cells were stained with fluorescein (fluorescein isothiocyanate, FITC)conjugated monoclonal antibodies against influenza A virus matrix protein 1 (M1) and nucleoprotein (NP). When these antibodies bind to their specific antigen the fluorescence intensity $(F)$ of the considered cell increases. There is no discrimination between the contributions of M1 and NP as all antibodies are labeled with the same fluorochrome. In addition, there is unspecific antibody binding which causes cells with no intracellular viral protein, like uninfected cells, to show fluorescence. As a result, for every sample time point a specific distribution over the fluorescence intensity is recorded, which characterizes the sampled cell population. Examples of experimental cell distributions obtained from flow cytometry are shown in Fig. 7.

The possibility of incorporating these flow cytometric results motivated the application of a distributed modeling approach in order to extend the lumped model of Möhler et al. [2005]. In the new model the intracellular amount of viral proteins M1 and NP should constitute the distinguishing feature and internal coordinate of the infected cells. Therefore, a degree of infection $(\delta \in \mathbb{R},[0, \infty])$ was introduced in a model published by Müller et al. [2008]. The degree of infection is a measure for the combined amount of intracellular M1 and NP given in virus equivalents (VE). A virus equivalent is the average number of copies of the considered protein(s) (in this case M1 and NP) which constitute a virus particle - according to Lamb and Krug [2001] an average influenza A virus particle contains 3000 copies of M1 and 1000 copies of NP. Thus, when an infected cell with $\delta=i$ synthesizes a combined number of 4000 copies of M1 and NP its state changes to $\delta=i+1$.

As mentioned above, all cells - even cells without intracellular viral protein, like uninfected cells - show fluorescence due to unspecific antibody binding. This leads to an initial distribution of the cell population over the fluorescence intensity. Hence, the specific fluorescence intensity of any given cell is determined by its intracellular amount of viral protein and its initial fluorescence intensity. This implies the introduction of a more general degree of fluorescence $\varphi$ as an internal coordinate of not only infected, but all cells.

How is the degree of fluorescence related to the measured fluorescence intensity of a specific cell? In Schulze-Horsel et al. [2008] it is shown that the change of the intracellular amount of viral protein is linearly linked to the change of fluorescence intensity. 

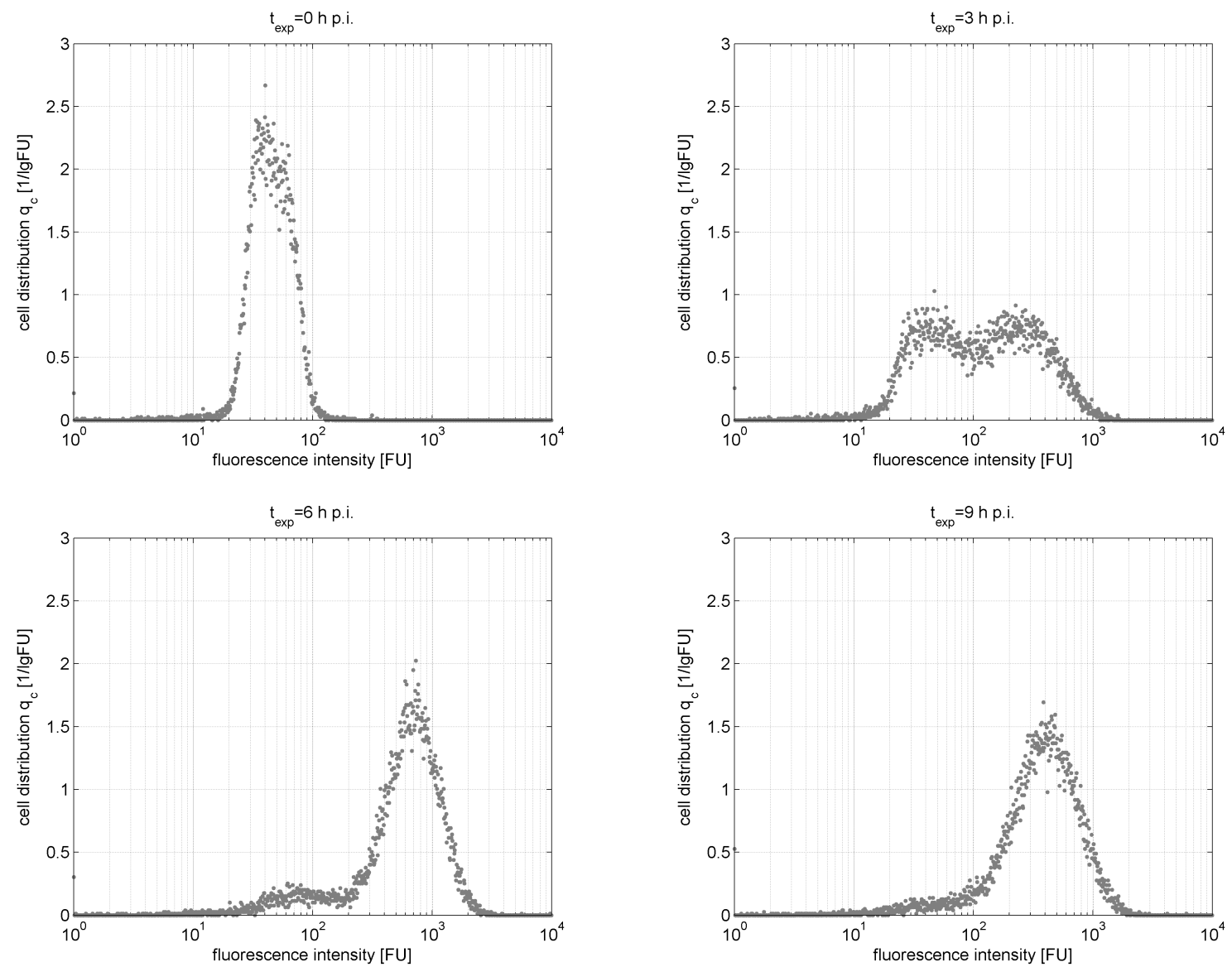

Figure 7: Experimental results obtained from flow cytometric measurements conducted by SchulzeHorsel et al. [2008] for the infection of adherent MDCK cells with equine influenza A/Newmarket/1/93 (H3N8). The diagrams comprise cell distributions of one experiment for selected times post infection.

Calibration measurements reveal that 3457.1 FITC molecules are needed to increase the fluorescence intensity by $1 \mathrm{FU}$ - the difference in the molar quantum yield between FITC conjugated to calibration particles and FITC conjugated to antibody molecules is neglected [ibid.]. Together with the labeling efficiency of 2.3 FITC molecules per viral protein [Schulze-Horsel, 2011] this yields the change in fluorescence intensity per virus equivalent

$$
F_{\mathrm{VE}}=4000 \text { proteins } \cdot 2.3 \frac{\mathrm{FITC}}{\text { protein }} \cdot \frac{1}{3457.1} \frac{\mathrm{FU}}{\mathrm{FITC}}=2.661 \mathrm{FU} \text {. }
$$

With the help of $F_{\mathrm{VE}}$ the degree of fluorescence can be defined as the fluorescence intensity of a specific cell divided by the change in fluorescence intensity per virus equivalent

$$
\varphi=F / F_{\mathrm{VE}} .
$$


Thus, when an infected cell with $\varphi=i$ synthesizes a combined number of 4000 copies of M1 and NP its state changes to $\varphi=i+1$. Though, because of the initial distribution of the cells due to unspecific antibody binding, $\varphi$-in contrast to $\delta$ - is not directly linked to the actual intracellular amount of viral protein of the considered cell.

\subsection{Preprocessing of flow cytometric data}

In order to provide initial conditions for the model and to allow the comparison with simulation results in general the flow cytometric data have to be preprocessed.

\section{Experimental cell distribution}

The flow cytometer possesses $N_{\text {sensor }}=1024$ sensor channels which cover a combined fluorescence intensity range from 1 to $10^{4}$ fluorescence units (FU). The lower and upper bound of each channel can be calculated from the channel number $i \in \mathbb{N}$, [1, 1024], which determines the width of the respective channel

$$
\begin{aligned}
& \Delta F_{\text {sensor }, i}=F_{\text {sensor }, i+1}-F_{\text {sensor }, i} \\
& =10^{\frac{4}{N_{\text {sensor }}} \cdot i}-10^{\frac{4}{N_{\text {sensor }}} \cdot(i-1)} \quad[\mathrm{FU}] .
\end{aligned}
$$

This leads to a logarithmically increasing channel width, so that in a logarithmic scale the width stays constant for all channels

$$
\begin{aligned}
\Delta F_{\text {sensor }, \mathrm{lg}} & =\lg \left(10^{\frac{4}{N_{\text {sensor }}} \cdot i}\right)-\lg \left(10^{\frac{4}{N_{\text {sensor }}} \cdot(i-1)}\right) \\
& =\frac{4}{N_{\text {sensor }}} i-\frac{4}{N_{\text {sensor }}}(i-1)=\frac{4}{N_{\text {sensor }}} \quad[\text { "lg FU"]. }
\end{aligned}
$$

During the measurement the cells of a sample are assigned to the appropriate channel matching their specific fluorescence intensity. This results in a histogram, in which the number of cells $Z_{i}$ is recorded for each channel (Fig. 8, left). To become comparable to simulation results the histogram data have to be normalized with respect to the total number of cells per sample and divided by the logarithmic width of the sensor channels. This leads to the experimental cell distribution (Fig. 8, right)

$$
q_{\exp , i}(t)=\frac{Z_{i}(t)}{\sum_{j} Z_{j}(t)} \cdot \frac{N_{\text {sensor }}}{4} \quad\left[\frac{1}{" \lg \mathrm{FU} "}\right] \text { for } i, j \in \mathbb{N},[1,1024] .
$$



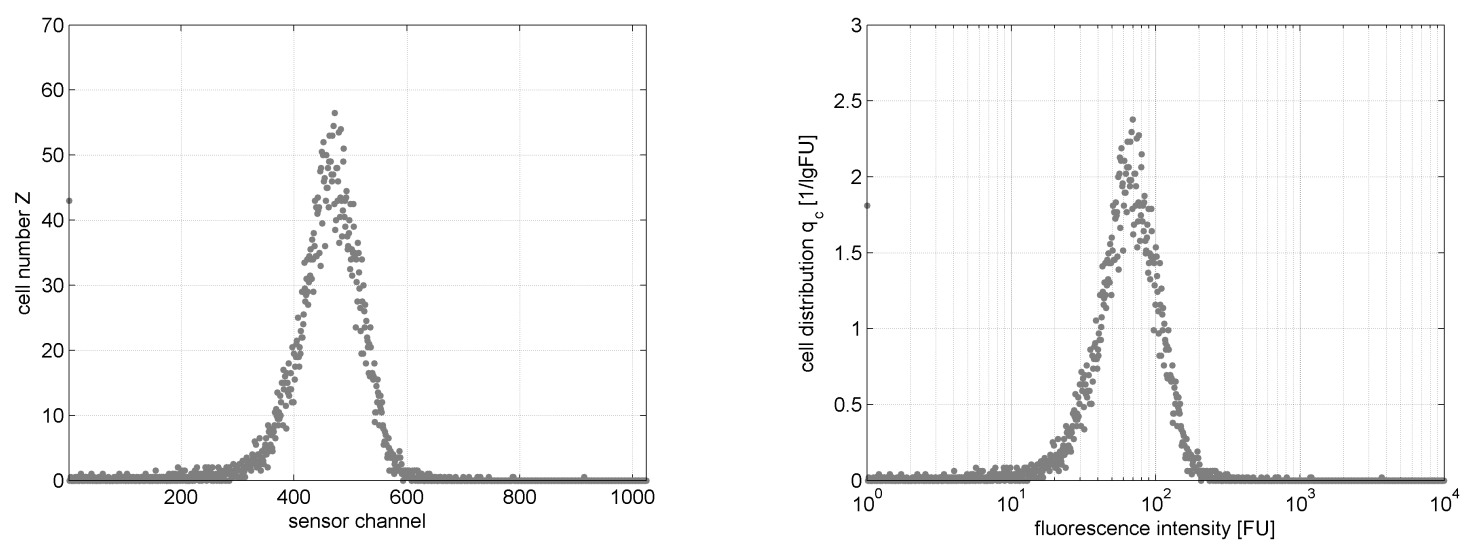

Figure 8: Flow cytometric data - raw cell counts for each sensor channel (left), experimental cell distribution $q_{\exp , i}$ over mean fluorescence intensity of respective sensor channel (right)

\section{Initial cell distribution}

The initial cell distribution is approximated by a log-normal distribution using the mean fluorescence intensity of the sensor channels $F_{\text {sensor }, m, i}$ :

$$
\begin{gathered}
q_{\text {approx }, i, 0}=\frac{1}{\sigma_{0} \sqrt{2 \pi}} \cdot \exp \left(-\frac{1}{2}\left[\frac{\lg \left(F_{\text {sensor }, \mathrm{m}, i} / \mu_{0}\right)}{\sigma_{0}}\right]^{2}\right) \quad\left[\frac{1}{" \lg \mathrm{FU} "}\right] \\
\text { with } F_{\text {sensor,m }, i}=\frac{1}{2}\left(10^{\frac{4}{N_{\text {sensor }}}} \cdot i+10^{\frac{4}{N_{\text {sensor }}} \cdot(i-1)}\right) \quad[\mathrm{FU}] .
\end{gathered}
$$

The expectation $\mu_{0}$ and the standard deviation $\sigma_{0}$ are fitted to experimental data. In Fig. 9 initial cell distributions are shown together with the respective experimental data recorded by Schulze-Horsel et al. [2008] for the infection of adherent MDCK cells with equine influenza A/Newmarket/1/93 (H3N8).

In order to determine the initial cell distribution for the model a generic discretization of the model equations into $N_{\mathrm{CV}}$ finite control volumes is assumed and a conversion into the correct units is performed:

$$
q_{\mathrm{sim}, i, 0}=\frac{1}{\sigma_{0} \sqrt{2 \pi}} \cdot \exp \left(-\frac{1}{2}\left[\frac{\lg \left(\varphi_{\mathrm{m}, i} F_{\mathrm{VE}} / \mu_{0}\right)}{\sigma_{0}}\right]^{2}\right) \cdot \frac{\lg \left(\varphi_{i+1} / \varphi_{i}\right)}{\varphi_{i+1}-\varphi_{i}} \quad[-]
$$

for $i \in \mathbb{N},\left[1, N_{\mathrm{CV}}\right]$.

Here, $\varphi_{\mathrm{m}, i}$ is the mean degree of fluorescence and $\varphi_{i}$ and $\varphi_{i+1}$ are the left and right boundaries of control volume (CV) $i$. 

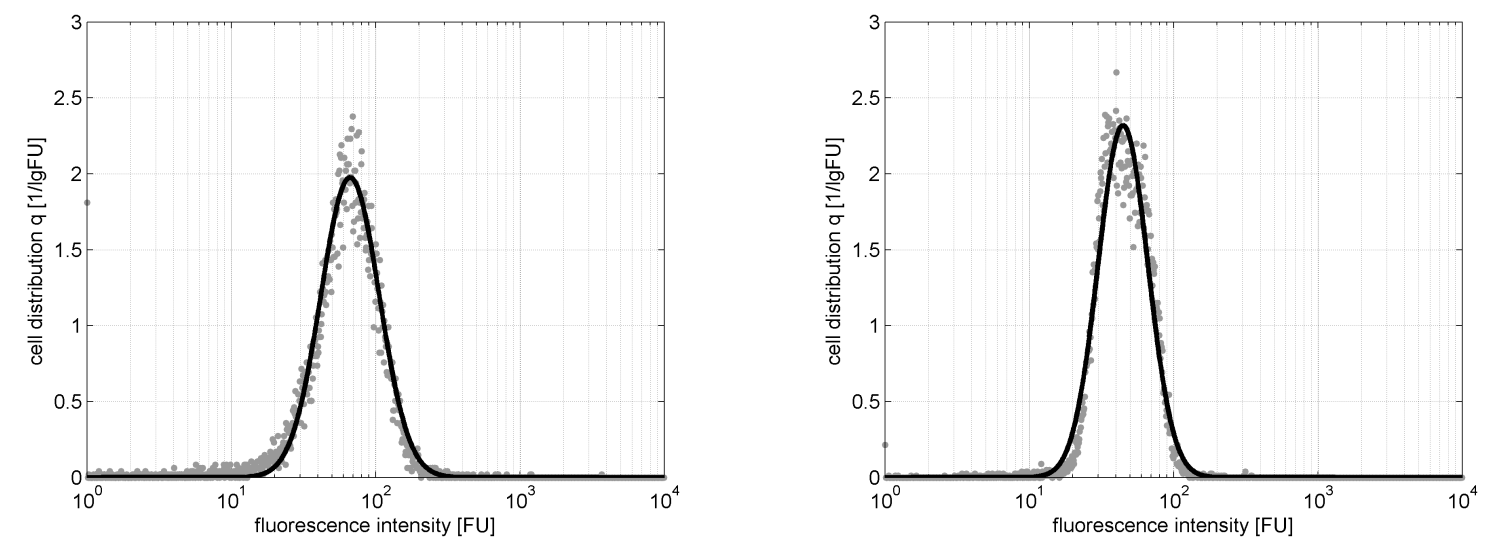

Figure 9: Initial cell distributions for two different experiments with equine influenza A/Newmarket/1/93 (H3N8) [Schulze-Horsel et al., 2008] - experimental cell distributions $q_{\exp , i, 0}(\mathbf{d o t s})$ and the respective approximated distributions $q_{\text {approx }, i, 0}$ (lines)

\section{Continuous formulation}

In order to obtain a continuous formulation of the initial cell distribution the scaling factor in Eq. (3.7) has to be adapted:

$$
\frac{\lg \left(\varphi_{i+1} / \varphi_{i}\right)}{\varphi_{i+1}-\varphi_{i}}=\frac{\lg \left(\left(\varphi_{i}+\Delta \varphi_{i}\right) / \varphi_{i}\right)}{\left(\varphi_{i}+\Delta \varphi_{i}\right)-\varphi_{i}}=\frac{\lg \left(1+\Delta \varphi_{i} / \varphi_{i}\right)}{\Delta \varphi_{i}} .
$$

Now, the limit of the scaling factor for $\Delta \varphi_{i} \rightarrow 0$ is determined with the help of L'Hospital's rule (see Appendix A, page 97):

$$
\lim _{\Delta \varphi_{i} \rightarrow 0} \frac{\lg \left(1+\Delta \varphi_{i} / \varphi_{i}\right)}{\Delta \varphi_{i}} \stackrel{!}{=} \lim _{\Delta \varphi_{i} \rightarrow 0} \frac{1}{\ln (10)\left(\varphi_{i}+\Delta \varphi_{i}\right)}=\frac{1}{\ln (10) \varphi_{i}} .
$$

Hence, the continuous formulation of the initial distribution is defined as

$$
q_{\mathrm{sim}, 0}(\varphi)=\frac{1}{\sigma_{0} \sqrt{2 \pi}} \cdot \exp \left(-\frac{1}{2}\left[\frac{\lg \left(\varphi F_{\mathrm{VE}} / \mu_{0}\right)}{\sigma_{0}}\right]^{2}\right) \cdot \frac{1}{\ln (10) \varphi} \quad[-]
$$

\subsection{Process description and model assumptions}

The general conditions of the considered process correspond to the ones described in Section 2.1. In addition, the intracellular production of M1 and NP has to be considered. It is assumed that the delay between the infection and the production of the first viral 
proteins, the so called eclipse period or eclipse phase [Flint et al., 2009], coincides with the latent phase of infected cells. All cells are considered to be distributed with respect to the fluorescence intensity. However, uninfected cells and infected cells which are currently in the latent phase are considered to keep their initial fluorescence intensity. In the measurements performed by Schulze-Horsel et al. [2008] the specific contributions of the two proteins M1 and NP to the overall fluorescence intensity are not discriminated. Hence, in the model both proteins are not discriminated either and their production is merged to a general production of viral protein. It is assumed that, besides unspecific antibody binding, the fluorescein-labeled antibodies only bind to newly synthesized viral proteins and not to proteins which were brought into the cell by the infecting virus particle. This assumption is supported by infection experiments with very high MOI, which were performed by the Bioprocess Engineering Group at the Max Planck Institute for Dynamics of Complex Technical Systems (MPI Magdeburg) (data not shown). The dynamics of virus protein accumulation are assumed to be exclusively controlled by the interplay of protein production and virus release. While the intracellular amount of viral protein is increased by protein production, the release of virus particles is considered to be eventually responsible for its decrease.

\subsection{Model equations}

The presented model is based on the approach previously published by Müller et al. [2008]. However, the latter is extended by explicit incorporation of the latent phase and the growth and death of uninfected cells. Therefore, the presented model shall be identified as the extended model of Müller et al. [2008]. As this distributed approach is conceived to be in accordance with the lumped model of Möhler et al. [2005] most equations have corresponding features when compared with the ones presented in Section 2.2. Note, that all state variables which are number densities are marked with a tilde (e.g. $\left.\tilde{U}_{\mathrm{c}}(t, \varphi), \tilde{I}_{\mathrm{c}}(t, \varphi)\right)$ in order to make them distinguishable from cell concentrations (e.g. $\left.U_{\mathrm{c}}(t), I_{\mathrm{c}}(t)\right)$.

In the beginning all cells are uninfected and distributed with respect to the degree of fluorescence:

$$
\begin{gathered}
\frac{\partial \tilde{U}_{\mathrm{c}}(t, \varphi)}{\partial t}=\mu_{\mathrm{c}}(t) \tilde{U}_{\mathrm{c}}(t, \varphi)-k_{\mathrm{cdf}} \tilde{U}_{\mathrm{c}}(t, \varphi)-k_{\mathrm{vi}} \tilde{U}_{\mathrm{c}}(t, \varphi) V(t) \\
\text { with } \quad \mu_{\mathrm{c}}(t)=\mu_{\mathrm{c}, \max } \frac{C_{\max }-\int_{\varphi}\left(\tilde{U}_{\mathrm{c}}(t, \varphi)+\tilde{L}_{\mathrm{c}}(t, \varphi)+\tilde{I}_{\mathrm{c}}(t, \varphi)\right) \mathrm{d} \varphi}{C_{\max }} .
\end{gathered}
$$

The initial condition is determined by the initial cell concentration $U_{\mathrm{c}, 0}$ and the normal- 
ized initial distribution $q_{\mathrm{sim}, 0}$ with the parameters $\mu_{0}$ and $\sigma_{0}$ (see Eq. (3.8)):

$$
\tilde{U}_{\mathrm{c}}(t=0, \varphi)=U_{\mathrm{c}, 0} \cdot q_{\mathrm{sim}, 0}(\varphi) .
$$

Note, uninfected cells are supposed to keep their degree of fluorescence.

The incorporation of the latent period is done in a different way than before. Infected cells which undergo the latent phase of duration $\tau$ are considered a separate species $\tilde{L}_{\mathrm{c}}$. Infected cells enter the latent phase upon infection and leave after the duration $\tau$. In addition, cells in the latent phase are supposed to be subject to cell death with the cell death coefficient due to viral infection $k_{\mathrm{cdv}}$. They are also considered to keep their degree of fluorescence.

$$
\frac{\partial \tilde{L}_{\mathrm{c}}(t, \varphi)}{\partial t}=k_{\mathrm{vi}} \tilde{U}_{\mathrm{c}}(t, \varphi) V(t)-k_{\mathrm{vi}} \tilde{U}_{\mathrm{c}}(t-\tau, \varphi) V(t-\tau) \cdot \mathrm{e}^{-k_{\mathrm{cdv}} \tau}-k_{\mathrm{cdv}} \tilde{L}_{\mathrm{c}}(t, \varphi)
$$

The first term on the right side of Eq. (3.12) represents cells entering the latent phase due to infection at time $t$. The second term specifies the change of the number density at time $t$ due to cells leaving the latent phase. As a matter of fact these cells must have entered the latent phase by virtue of infection at time $t-\tau$. Though, due to cell death their number decreases during their stay in the latent phase until they leave at time $t$. This decrease is reflected by an exponential expression depending on the cell death coefficient $k_{\mathrm{cdv}}$ and the length of the latent phase $\tau$. As a result, the number of cells leaving the latent phase at time $t$ ends up to be smaller than the number of cells which originally entered the latent phase at time $t-\tau$. Finally, the third term represents cells dying at time $t$. The derivation of Eq. (3.12) is shown in detail in Appendix B (page $99)$.

After the latent phase is completed, infected cells start to produce viral proteins M1 and $\mathrm{NP}$ with the protein production coefficient $k_{\text {pro }}$, which causes the degree of fluorescence to increase. On the other hand, virus particles are released with the release coefficient $k_{\text {rel }}$, which causes the degree of fluorescence to decrease. The interplay of these two coefficients determines the distribution dynamics:

$$
\begin{aligned}
\frac{\partial \tilde{I}_{\mathrm{c}}(t, \varphi)}{\partial t}= & k_{\mathrm{vi}} \tilde{U}_{\mathrm{c}}(t-\tau, \varphi) V(t-\tau) \cdot \mathrm{e}^{-k_{\mathrm{cdv}} \tau}-\left(k_{\mathrm{pro}}-k_{\mathrm{rel}}\right) \frac{\partial \tilde{I}_{\mathrm{c}}(t, \varphi)}{\partial \varphi} \\
& -k_{\mathrm{cdv}} \tilde{I}_{\mathrm{c}}(t, \varphi) .
\end{aligned}
$$

By explicitly considering cells in the latent phase as a separate species, all cells that have left the latent phase are immediately capable of virus replication. Hence, virus particles are instantly released by infected cells of species $\tilde{I}_{\mathrm{C}}$ :

$$
\frac{\mathrm{d} V(t)}{\mathrm{d} t}=k_{\mathrm{rel}} \int_{\varphi} \tilde{I}_{\mathrm{c}}(t, \varphi) \mathrm{d} \varphi-k_{\mathrm{vd}} V(t)-k_{\mathrm{va}} V(t) \int_{\varphi} \tilde{U}_{\mathrm{c}}(t, \varphi) \mathrm{d} \varphi .
$$




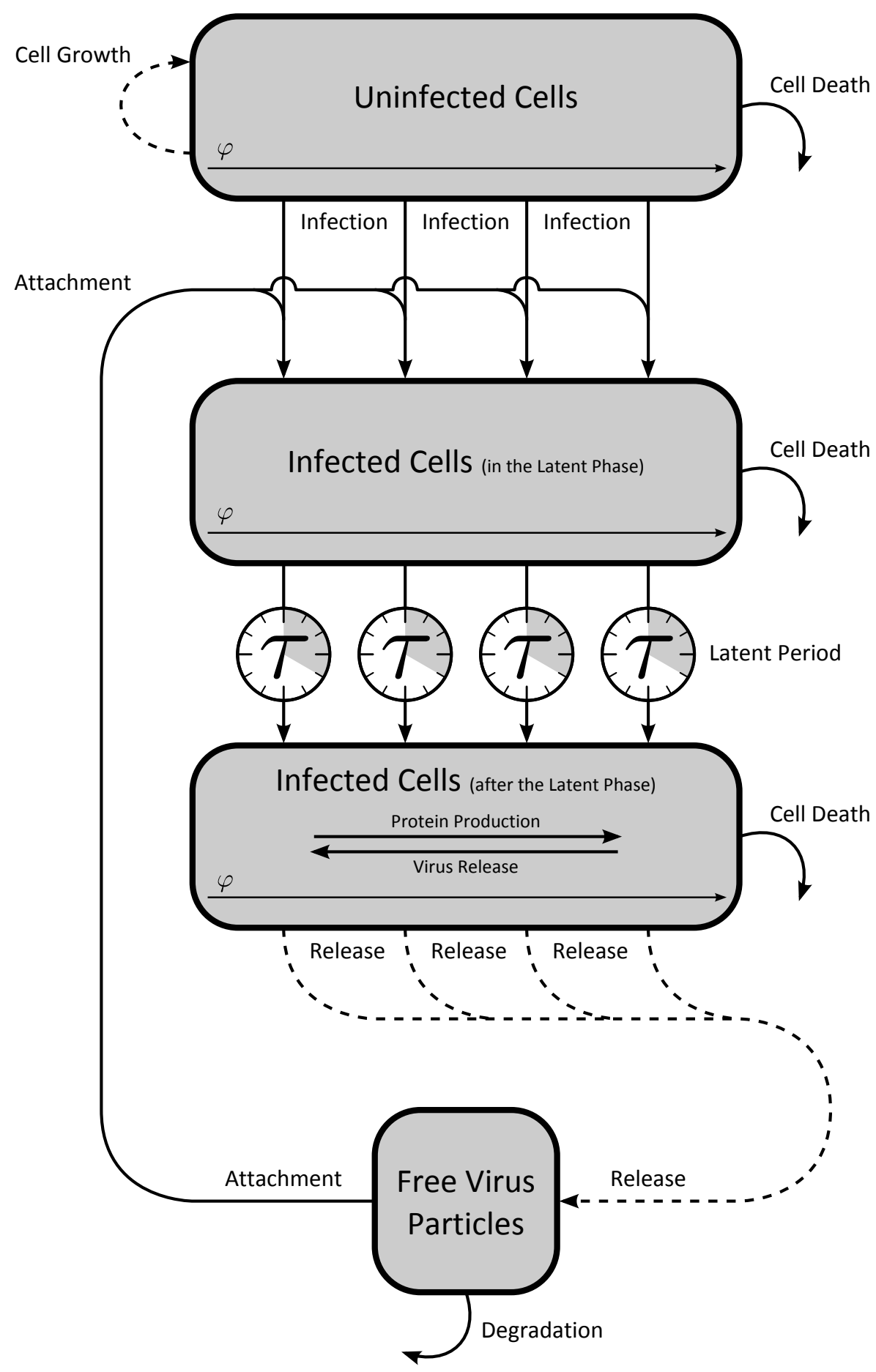

Figure 10: Scheme of the extended model of Müller et al. [2008]. Uninfected cells and infected cells (in and after the latent phase) are distributed over the degree of fluorescence $\varphi$. While uninfected cells keep their degree of fluorescence, infected cells - once they have passed the latent phase - can move along the internal coordinate $\varphi$ by means of viral protein production and virus release. 
The resulting model structure is presented in Fig. 10.

\subsection{Numerical solution}

For the numerical solution some of the model equations presented above can be simplified. Note, that by assumption uninfected cells and infected cells during the latent phase do not change their degree of fluorescence. Therefore, the number densities $\tilde{U}_{\mathrm{c}}(t, \varphi)$ and $\tilde{L}_{\mathrm{c}}(t, \varphi)$ can be determined by multiplying the respective cell concentrations $U_{\mathrm{c}}(t)$ and $L_{\mathrm{c}}(t)$ with the time invariant normalized initial distribution $q_{\mathrm{sim}, 0}(\varphi)$ (see Eq. (3.8) on page 21):

$$
\begin{aligned}
& \tilde{U}_{\mathrm{c}}(t, \varphi)=U_{\mathrm{c}}(t) q_{\mathrm{sim}, 0}(\varphi) \quad \text { with } \quad U_{\mathrm{c}}(t)=\int_{\varphi} \tilde{U}_{\mathrm{c}}(t, \varphi) \mathrm{d} \varphi \\
& \tilde{L}_{\mathrm{c}}(t, \varphi)=L_{\mathrm{c}}(t) q_{\mathrm{sim}, 0}(\varphi) \quad \text { with } \quad L_{\mathrm{c}}(t)=\int_{\varphi} \tilde{L}_{\mathrm{c}}(t, \varphi) \mathrm{d} \varphi .
\end{aligned}
$$

Hence, for the uninfected cells application of Eq. (3.15) to Eq. (3.9) yields the temporal change of the concentration, which corresponds to Eq. (2.1) on page 10:

$$
\frac{\partial U_{\mathrm{c}}(t)}{\partial t}=\mu_{\mathrm{c}}(t) U_{\mathrm{c}}(t)-k_{\mathrm{cdf}} U_{\mathrm{c}}(t)-k_{\mathrm{vi}} U_{\mathrm{c}}(t) V(t) .
$$

As demonstrated previously in Appendix B, for the numerical solution the latent phase is represented by a transport system using the new state variable $\hat{\tilde{L}}_{\mathrm{c}}$, which depends on the degree of fluorescence $\varphi$ and the transport coordinate $\lambda$ (see Eq. (B.1) on page 99). Then, in correspondence to Eq. (3.16) the following holds

$$
\hat{\tilde{L}}_{\mathrm{c}}(t, \varphi, \lambda)=\hat{L}_{\mathrm{c}}(t, \lambda) q_{\mathrm{sim}, 0}(\varphi) \quad \text { with } \quad \hat{L}_{\mathrm{c}}(t, \lambda)=\int_{\varphi} \hat{\tilde{L}}_{\mathrm{c}}(t, \varphi, \lambda) \mathrm{d} \varphi
$$

and can be applied to Eq. (B.1) yielding the balance equation of the number density of the infected cells in the latent phase at position $\lambda$ :

$$
\frac{\partial \hat{L}_{\mathrm{c}}(t, \lambda)}{\partial t}=-\frac{1}{\tau} \frac{\partial \hat{L}_{\mathrm{c}}(t, \lambda)}{\partial \lambda}-k_{\mathrm{cdv}} \hat{L}_{\mathrm{c}}(t, \lambda)
$$

with the boundary condition

$$
\hat{L}_{\mathrm{c}}(t, \lambda=0)=\tau \cdot k_{\mathrm{vi}} U_{\mathrm{c}}(t) V(t) .
$$

Subsequently, Eq. (3.18) is discretized with the help of the finite-volume method (FVM) using an equidistant discretization scheme with $M=64$ control volumes (see Appendix 
C.2 on page 102):

$$
\begin{gathered}
\frac{\mathrm{d} \hat{L}_{\mathrm{c}, i}(t)}{\mathrm{d} t}=-\frac{1}{\Delta \lambda \tau}\left(\hat{L}_{\mathrm{c}, i}(t)-\hat{L}_{\mathrm{c}, i-1}(t)\right)-k_{\mathrm{cdv}} \hat{L}_{\mathrm{c}, i}(t) \\
\text { with } \quad \hat{L}_{\mathrm{c}, 0}(t)=\tau \cdot k_{\mathrm{vi}} U_{\mathrm{c}}(t) V(t) .
\end{gathered}
$$

When looking at the last control volume of Eq. (3.20) $(i=M)$ the change of the cell concentration due to cells leaving the latent phase at time $t$ equals $\hat{L}_{\mathrm{c}, M}(t) / \tau$. By multiplication with the normalized initial distribution $q_{\text {sim,o }}(\varphi)$ this flux serves as the source of infected cells of species $\tilde{I}_{\mathrm{c}}(t, \varphi)$. The balance equation of infected cells after the latent phase, Eq. (3.13), is changed accordingly:

$$
\frac{\partial \tilde{I}_{\mathrm{c}}(t, \varphi)}{\partial t}=\frac{\hat{L}_{\mathrm{c}, M}(t)}{\tau} q_{\mathrm{sim}, 0}(\varphi)-\left(k_{\mathrm{pro}}-k_{\mathrm{rel}}\right) \frac{\partial \tilde{I}_{\mathrm{c}}(t, \varphi)}{\partial \varphi}-k_{\mathrm{cdv}} \tilde{I}_{\mathrm{c}}(t, \varphi) .
$$

Now, Eq. (3.21) is discretized by FVM using a logarithmic discretization scheme with $N=128$ control volumes (see Appendix C.3, page 104):

$$
\begin{aligned}
\frac{\mathrm{d} \tilde{I}_{\mathrm{c}, i}(t)}{\mathrm{d} t}= & \frac{\hat{L}_{\mathrm{c}, M}(t)}{\tau} q_{\mathrm{sim}, 0, i}-\frac{k_{\mathrm{pro}}}{\Delta \varphi_{i}}\left(\tilde{I}_{\mathrm{c}, i}(t)-\tilde{I}_{\mathrm{c}, i-1}(t)\right) \\
& +\frac{k_{\mathrm{rel}}}{\Delta \varphi_{i}}\left(\tilde{I}_{\mathrm{c}, i+1}(t)-\tilde{I}_{\mathrm{c}, i}(t)\right)-k_{\mathrm{cdv}} \tilde{I}_{\mathrm{c}, i}(t)
\end{aligned}
$$

$$
\text { with } \quad \tilde{I}_{\mathrm{c}, 0}=\tilde{I}_{\mathrm{c}, N+1}=0 .
$$

In addition, convection across the boundaries is prevented by defining $I_{\mathrm{c}, N}=0$ for the protein production term and $I_{\mathrm{c}, 1}=0$ for the virus release term.

Due to the reformulation of the balance equations of all cell species the virus balance equation and the equation of the cell growth rate have to be adapted accordingly:

$$
\begin{aligned}
& \frac{\mathrm{d} V(t)}{\mathrm{d} t}=k_{\mathrm{rel}} \sum_{i=1}^{N} \Delta \varphi_{i} \tilde{I}_{\mathrm{c}, i}(t)-k_{\mathrm{vd}} V(t)-k_{\mathrm{va}} U_{\mathrm{c}}(t) V(t), \\
& \mu_{\mathrm{c}}(t)=\mu_{\mathrm{c}, \max } \frac{C_{\max }-U_{\mathrm{c}}(t)-\Delta \lambda \sum_{i=1}^{M} \hat{L}_{\mathrm{c}, i}(t)-\sum_{i=1}^{N} \Delta \varphi_{i} \tilde{I}_{\mathrm{c}, i}(t)}{C_{\max }} .
\end{aligned}
$$

The resulting model structure is presented in Fig. 11. 


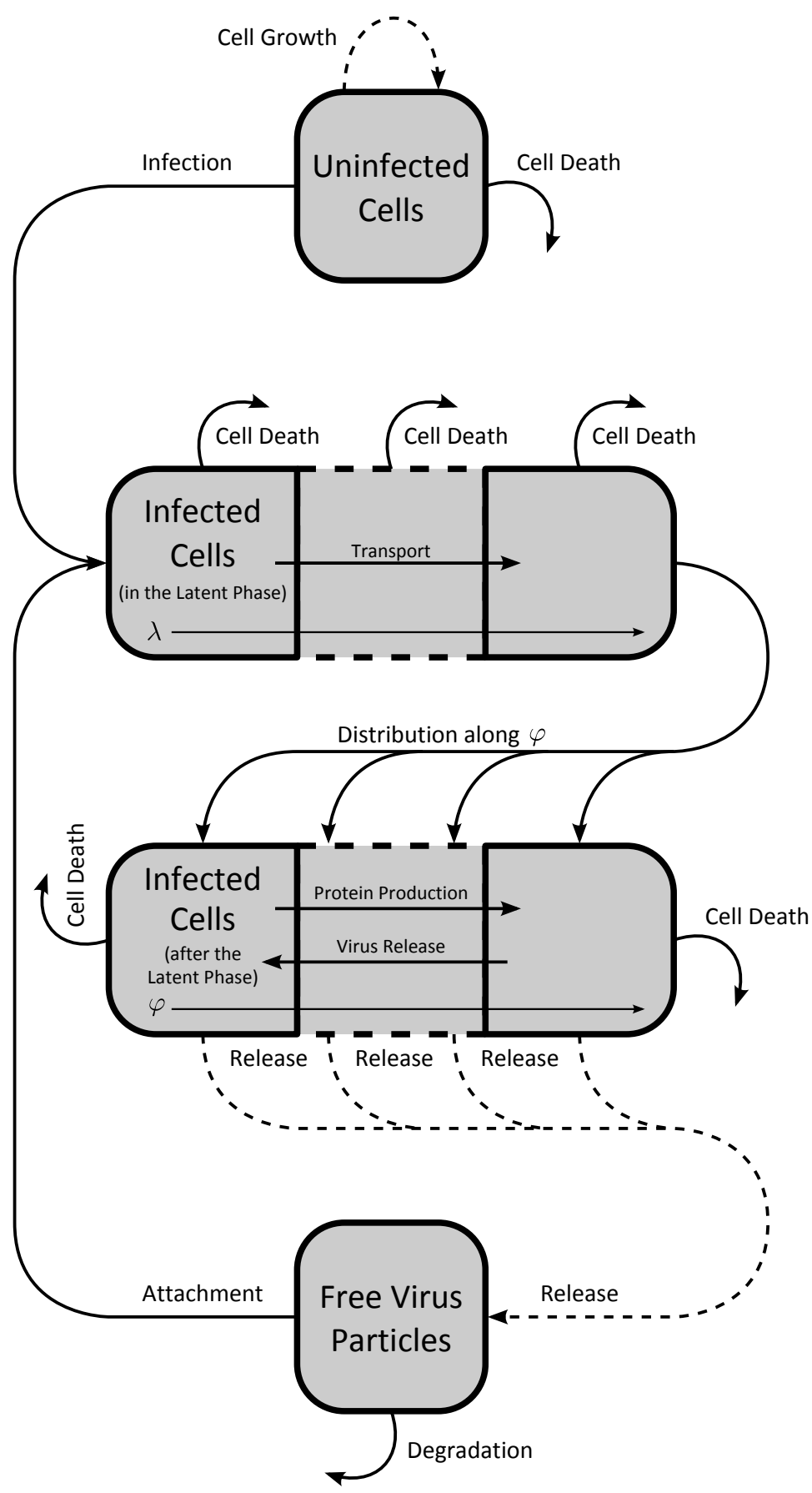

Figure 11: Scheme of the discretized, extended model of Müller et al. [2008]. Uninfected cells and infected cells in the latent phase are assumed to not change their degree of fluorescence $\varphi$. Therefore, they can be implemented as lumped species with regard to $\varphi$. The latent phase is approximated by a discretized transport system with the transport coordinate $\lambda$. When cells in the latent phase reach the end of the transport system they change over to the virus producing species of infected cells. By applying the normalized initial cell distribution, infected cells which have passed the latent phase are distributed along $\varphi$. 


\begin{tabular}{|c|c|c|c|c|}
\hline \multirow[t]{2}{*}{ Parameter } & \multicolumn{3}{|c|}{ MOI } & \multirow[t]{2}{*}{ Unit } \\
\hline & 0.025 & 1.0 & 3.0 & \\
\hline$C_{\max }$ & & $1.2 \cdot 10^{6}$ & & $\mathrm{ml}^{-1}$ \\
\hline$k_{\text {cdf }}$ & & 0.001 & & $h^{-1}$ \\
\hline$k_{\mathrm{cdv}}$ & & $25.7 \cdot 10^{-3}$ & & $\mathrm{~h}^{-1}$ \\
\hline$k_{\text {pro }}$ & 247 & 299 & 120 & $\mathrm{~h}^{-1}$ \\
\hline$k_{\text {rel }}$ & 246 & 288 & 110 & $\mathrm{~h}^{-1}$ \\
\hline$k_{\mathrm{va}}$ & & 0.8 & & $\mathrm{ml} / \mathrm{h}$ \\
\hline$k_{\mathrm{vd}}$ & & 0.009 & & $h^{-1}$ \\
\hline$k_{\mathrm{vi}}$ & $1.9 \cdot 10^{-3}$ & $1.9 \cdot 10^{-3}$ & $0.71 \cdot 10^{-3}$ & $\mathrm{ml} / \mathrm{h}$ \\
\hline$\mu_{\mathrm{c}, \max }$ & & 0.03 & & $\mathrm{~h}^{-1}$ \\
\hline$\tau$ & 0.7 & 2.2 & 5 & $\mathrm{~h}$ \\
\hline$I_{\mathrm{c}, 0}$ & & 0 & & $\mathrm{ml}^{-1}$ \\
\hline$U_{\mathrm{c}, 0}$ & & $1.2 \cdot 10^{6}$ & & $\mathrm{ml}^{-1}$ \\
\hline$V_{0}$ & $12.6 \cdot 10^{6}$ & $502.4 \cdot 10^{6}$ & $1.507 \cdot 10^{9}$ & $\mathrm{ml}^{-1}$ \\
\hline$\mu_{0}$ & 66.5 & 44.6 & 57.6 & $\mathrm{FU}$ \\
\hline$\sigma_{0}$ & 0.202 & 0.172 & 0.159 & FU \\
\hline
\end{tabular}

Table II: Parameter sets and initial conditions for the extended model of Müller et al. [2008]. Parameters that are not adopted from Tab. I (marked in gray) were fitted to experimental data from Schulze-Horsel et al. [2008].

\subsection{Parameters}

The previously described population balance model is compared with experimental data collected by Schulze-Horsel et al. [2008] for the production of equine influenza A/Newmarket/1/93 (H3N8) in MDCK microcarrier culture in a small-scale bioreactor (11 working volume) at three different MOI $(0.025,1.0$, and 3.0). In the following, the considered virus strain will be referred to as A/Equi/H3N8. The experimental setting is similar to the one employed by Möhler et al. [2005] - in both publications the same virus strain and cell line are used; both refer to the same cultivation procedures, which were established previously by Genzel et al. [2004]. Therefore, most of the model parameters of Tab. I can be adopted. The applied parameter set and initial conditions for all three MOI are summarized in Tab. II. Parameters that were fitted to the experimental data of Schulze-Horsel et al. [2008] are marked in gray. In the following, the process of how these parameters are derived is explained.

In order to determine the initial conditions it is important to regard the differences between Schulze-Horsel et al. [2008] and Möhler et al. [2005] when it comes to calculating the total virus concentration from virus titers measured via hemagglutination (HA) 
assay. While the latter assume that at least two virus particles per red blood cell (RBC) are needed to cause complete agglutination, the former - referring to Burleson et al. [1992] - consider one virus particle per RBC enough to produce the same result. Hence, in accordance with Schulze-Horsel et al. [2008] and Schulze-Horsel [2011] the total virus concentration can be calculated from virus titers measured in logarithmic HA units per testvolume $\left(\frac{\lg \mathrm{HA}}{100 \mathrm{pl}}\right)$ :

$$
\begin{gathered}
V=c_{\mathrm{RBC}} \cdot 10^{\frac{\operatorname{lg~HA}}{100 \mathrm{ul}}} \quad[\text { virusparticles } / \mathrm{ml}] \\
\text { with } \quad c_{\mathrm{RBC}}=2 \cdot 10^{7} \mathrm{RBC} / \mathrm{ml}
\end{gathered}
$$

Accordingly, the detection limit of the method equals the concentration of red blood cells in the erythrocyte suspension $\left(c_{\mathrm{RBC}}\right)$ and amounts to $2 \cdot 10^{7}$ virusparticles $/ \mathrm{ml}$. The used virus seed is characterized by a total virus titer of $2.4 \frac{\lg \mathrm{HA}}{100 \mathrm{\mu l}}$ and an infectious virus titer of $1.2 \cdot 10^{7}$ plaque forming units (PFU) per ml [Genzel et al., 2004], which corresponds to a total virus concentration of $V_{\text {seed }}=5 \cdot 10^{9}$ virusparticles $/ \mathrm{ml}$ and an infectious virus concentration of $V_{\text {inf,seed }}=1.2 \cdot 10^{7}$ virusparticles $/ \mathrm{ml}$. Hence, the plating efficiency $\left(P_{\text {eff }}\right)$ of the virus seed can be calculated as

$$
P_{\text {eff }}=\frac{V_{\text {inf,seed }}}{V_{\text {seed }}}=2.4 \cdot 10^{-3} \text {. }
$$

Thus, the initial virus concentration $\left(V_{0}\right)$ is determined by the given MOI and the initial concentration of uninfected cells

$$
V_{0}=\frac{\mathrm{MOI}}{P_{\text {eff }}} U_{\mathrm{c}, 0}
$$

The initial cell distributions are approximated as described in Section 3.1 (page 19 ff.). Expectation and standard deviation $\left(\mu_{0}\right.$ and $\left.\sigma_{0}\right)$ are fitted to the respective experimental cell distributions by minimizing error squares.

Of the remaining four parameters three have influence on the integral dynamics without being influenced by the distribution dynamics. These are the infection coefficient $k_{\mathrm{vi}}$, the duration of the latent phase $\tau$, and the virus release coefficient $k_{\text {rel }}$. All are fitted to HA measurements by minimizing error squares.

First, the infection coefficient $k_{\mathrm{vi}}$ is determined by exploiting, that, within certain limits, the actual value of $k_{\mathrm{vi}}$ has only minor influence on the overall system dynamics as long as the ratio between $k_{\mathrm{vi}}$ and the attachment coefficient $k_{\mathrm{va}}$ is preserved - this is exemplified in the last paragraph of Section 2.3 (also see Fig. 5 on page 13). Then, the plating efficiency can be used as a measure for the ratio between $k_{\mathrm{vi}}$ and $k_{\mathrm{va}}$, so that:

$$
k_{\mathrm{vi}}=P_{\mathrm{eff}} \cdot k_{\mathrm{va}} \text {. }
$$




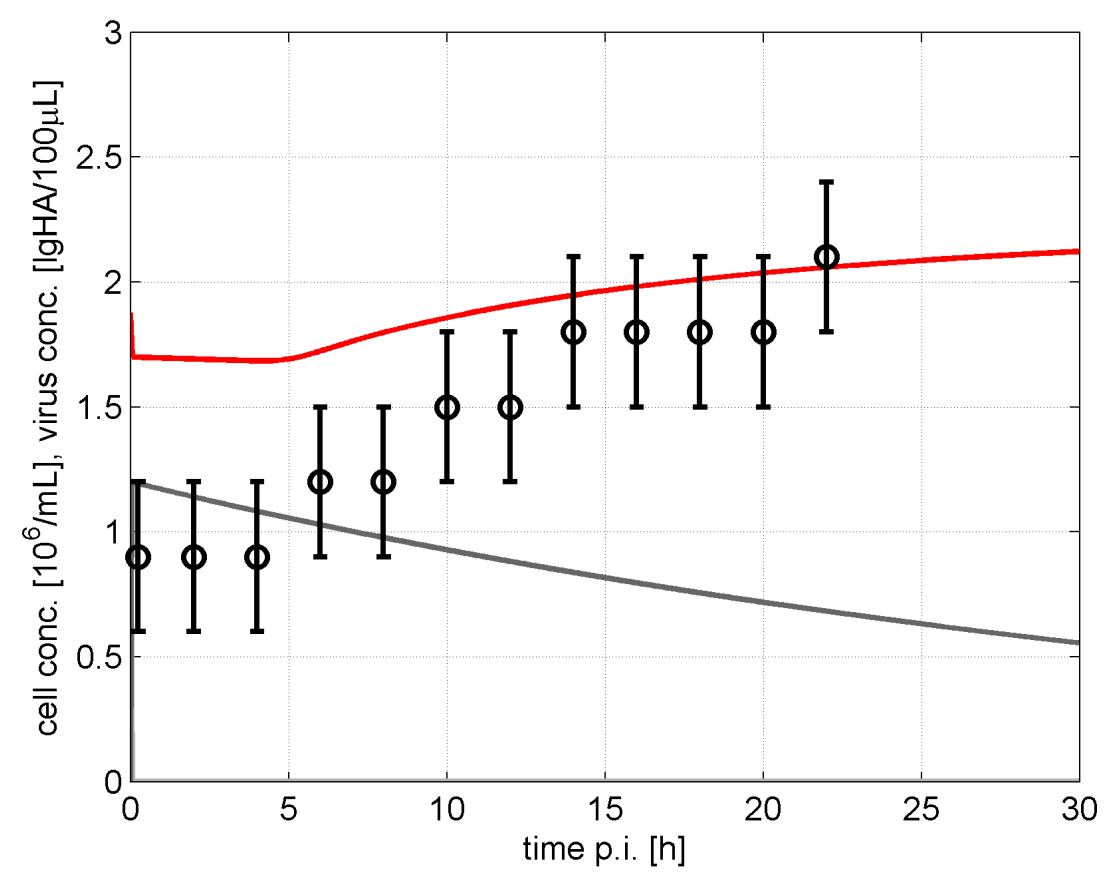

Figure 12: Comparison of the integral dynamics of the extended model of Müller et al. [2008] with experimental data of Schulze-Horsel et al. [2008] for MOI = 3.0 - lines represent simulation results for the concentration of uninfected cells (light gray), infected cells (sum of cells in and after the latent phase, gray), and virus particles (red); circles represent experimental virus titers measured via hemagglutination assay including standard error bars of the method $\left( \pm 0.3 \frac{\mathrm{lg} \mathrm{HA}}{100 \mathrm{\mu l}}\right)$. With exception of the infection coefficient $\left(k_{\mathrm{vi}}=1.9 \cdot 10^{-3} \mathrm{ml} / \mathrm{h}\right)$ the applied parameters are summarized in Tab. II (page 28). As the MOI is rather high all cells are infected instantly. However, there are far too much virus particles left after all cells are infected to provide a good fit to the experimental data.

Hence, with $k_{\mathrm{va}}=0.8 \mathrm{ml} / \mathrm{h} k_{\mathrm{vi}}$ is set to $1.9 \cdot 10^{-3} \mathrm{ml} / \mathrm{h}$. For MOI $=0.025$ and 1.0 this value of $k_{\mathrm{vi}}$ provides a good fit to the experimental data (see Fig. 13, page 33).

However, for MOI $=3.0$ the simulation results seem to be far off when $k_{\mathrm{vi}}=$ $1.9 \cdot 10^{-3} \mathrm{ml} / \mathrm{h}$ is applied (see Fig. 12). One reason for this discrepancy between simulation and experiment could be, that the model does not consider multiple infections. If virus particles would attach to already infected cells, as they surely do in reality, the virus concentration could decrease even when there are no uninfected cells left. However, whether the inclusion of multiple infections alone would be enough to bridge a gap this large is debatable. Another possible cause for the encountered difference could be an incorrect initial virus concentration in the experiment with $\mathrm{MOI}=3.0$. But that would mean, that less than half of the intended virus seed had been applied, which is rather unlikely. Yet, another explanation could be that the concentration of infectious virus particles in the virus seed $\left(V_{\text {inf,seed }}\right)$ was actually smaller than the denoted 
$1.2 \cdot 10^{7}$ virusparticles $/ \mathrm{ml}$, which directly translates into different values of $P_{\text {eff. }}$ This can happen, for instance, when defective interfering virus particles (DIPs) accumulate during passing processes of the virus before the actual experiment. Whatever the reasons are for the observed discrepancy, in the framework of the model the best fit for the experiment with $\mathrm{MOI}=3.0$ is achieved by setting the infection coefficient $k_{\mathrm{vi}}$ to $0.71 \cdot 10^{-3} \mathrm{ml} / \mathrm{h}$ (see Fig. 13, page 33). Note, that the independent determination of $k_{\mathrm{vi}}$ is possible because, under the given conditions, $k_{\mathrm{vi}}$ is the only remaining parameter that defines the initial decrease of the virus concentration, when the seed virus interacts with the cell population.

After $k_{\mathrm{vi}}$ has been set, the subsequent course of the virus concentration is determined by the duration of the latent phase $\tau$ and by the virus release coefficient $k_{\text {rel }}$. While $\tau$ sets the time when the first freshly produced virus particles are released, $k_{\text {rel }}$ determines the increase of the virus concentration - both parameters are fitted together.

In general, the values of $k_{\text {rel }}$ are smaller than the ones applied by Möhler et al. [2005]. This can be attributed directly to the differences in the way the total virus concentration is linked to the virus titer measured in $\frac{\lg \mathrm{HA}}{100 \mathrm{pl}}$ (see Eq. (3.25)). In comparison to the approach of Möhler et al. [2005] a given virus concentration corresponds to a higher HA value, so that the release rate can be lower in order to fit the experimental results. Note, in case of $\mathrm{MOI}=3.0$ the value of $k_{\text {rel }}$ is significantly lower than for the other two experiments. The impeded virus release is in agreement with the hypothesis about the increased occurrence of DIPs in this experiment.

Note, that the parameter $\tau$ subsumes various effects, which otherwise are not explicitly addressed by the model. Besides the processes which take place during the latent phase, $\tau$ is influenced by, e.g., the flow regime in the bioreactor, the level of trypsin in the medium, the occurrence of DIPs, and the detection limits of the applied assays. Thus, $\tau$ presumably is subject to significant variation depending on the various boundary conditions of the respective experiment. However, in the presented results $\tau$ appears to be proportional to the MOI or the initial virus concentration $V_{0}$, respectively.

Finally, the viral protein production coefficient $k_{\text {pro }}$, which, together with the already set $k_{\text {rel }}$, determines the distribution dynamics, is fitted by minimizing the error between the mean fluorescence intensities (MFIs) of simulated and experimental cell distributions. The MFI of the experimental cell distributions is determined by the normalized number of cells assigned to sensor channel $i$ and the mean fluorescence intensity of sensor channel $i$ (see Eqs. (3.5) and (3.6)):

$$
\mathrm{MFI}_{\exp }(t)=\frac{\sum_{i} Z_{i}(t) F_{\text {sensor,m }, i}}{\sum_{i} Z_{i}(t)} \quad[F U] \quad \text { for } i \in \mathbb{N},[1,1024] .
$$


The MFI of the simulated cell population is calculated in an analogous manner:

$$
\operatorname{MFI}_{\mathrm{sim}}(t)=\frac{\int_{\varphi}\left(\tilde{U}_{\mathrm{c}}(t, \varphi)+\tilde{L}_{\mathrm{c}}(t, \varphi)+\tilde{I}_{\mathrm{c}}(t, \varphi)\right) \varphi \mathrm{d} \varphi}{\int_{\varphi}\left(\tilde{U}_{\mathrm{c}}(t, \varphi)+\tilde{L}_{\mathrm{c}}(t, \varphi)+\tilde{I}_{\mathrm{c}}(t, \varphi)\right) \mathrm{d} \varphi} F_{\mathrm{VE}} \quad[F U] .
$$

For the numerical solution the calculation of the MFI changes due to the discretization scheme (see Section 3.4 (page 25 f.) and Appendices C.2 \& C.3 (page 102 ff.)):

$$
\begin{gathered}
\operatorname{MFI}_{\mathrm{sim}}(t) \approx \frac{\left(\left(U_{\mathrm{c}}(t)+\Delta \lambda \sum_{j=1}^{M} \hat{L}_{\mathrm{c}, j}(t)\right) \sum_{i=1}^{N} q_{\mathrm{sim}, 0, i}+\sum_{i=1}^{N} \tilde{I}_{\mathrm{c}, i}(t)\right) \varphi_{\mathrm{m}, i} \Delta \varphi_{i}}{U_{\mathrm{c}}(t)+\Delta \lambda \sum_{j=1}^{M} \hat{L}_{\mathrm{c}, j}(t)+\sum_{i=1}^{N} \tilde{I}_{\mathrm{c}, i}(t) \Delta \varphi_{i}} F_{\mathrm{VE}} \quad[F U] \\
\text { with } \Delta \lambda \sum_{j=1}^{M} \hat{L}_{\mathrm{c}, j}(t) \approx L_{\mathrm{c}}(t)=\int_{\varphi} \tilde{L}_{\mathrm{c}}(t, \varphi) \mathrm{d} \varphi \\
\sum_{i=1}^{N} \tilde{I}_{\mathrm{c}, i}(t) \Delta \varphi_{i} \approx I_{\mathrm{c}}(t)=\int_{\varphi} \tilde{I}_{\mathrm{c}}(t, \varphi) \mathrm{d} \varphi \\
U_{\mathrm{c}}(t) \sum_{i=1}^{N} q_{\mathrm{sim}, 0, i} \varphi_{\mathrm{m}, i} \Delta \varphi_{i} \approx \int_{\varphi} \tilde{U}_{\mathrm{c}}(t, \varphi) \varphi \mathrm{d} \varphi, \text { and } \\
\Delta \lambda \sum_{j=1}^{M} \hat{L}_{\mathrm{c}, j}(t) \sum_{i=1}^{N} q_{\mathrm{sim}, 0, i} \varphi_{\mathrm{m}, i} \Delta \varphi_{i} \approx L_{\mathrm{c}}(t) \sum_{i=1}^{N} q_{\mathrm{sim}, 0, i} \varphi_{\mathrm{m}, i} \Delta \varphi_{i} \\
\approx \int_{\varphi} \tilde{L}_{\mathrm{c}}(t, \varphi) \varphi \mathrm{d} \varphi .
\end{gathered}
$$

In the equations above $\varphi_{\mathrm{m}, i}$ is the mean fluorescence intensity and $\Delta \varphi_{i}$ the width of control volume $i$.

\subsection{Simulation results}

First, the integral dynamics are presented in comparison to experimental data collected by Schulze-Horsel et al. [2008] for the infection of adherent MDCK cells with $\mathrm{A} /$ Equi/H3N8 at MOI $=0.025,1.0$, and 3.0. Afterwards, the internal distribution dynamics are compared with flow cytometric measurements of the same experiments. The applied parameter set and initial conditions are summarized in Tab. II (page 28). 


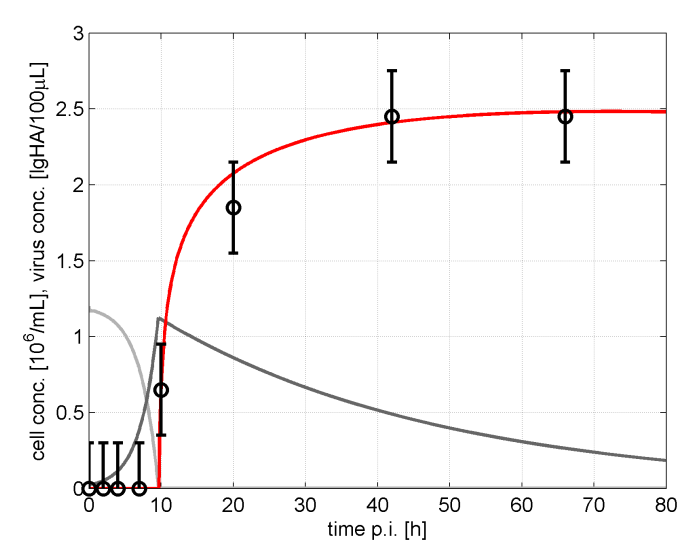

a) $\mathrm{MOI}=0.025$

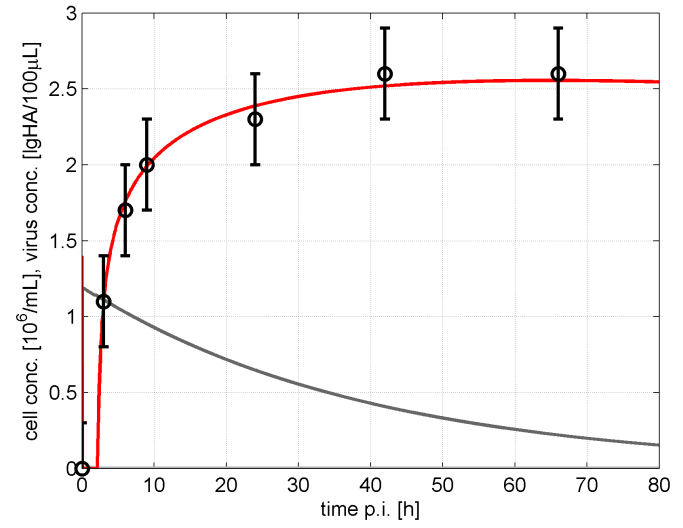

b) $\mathrm{MOI}=1.0$

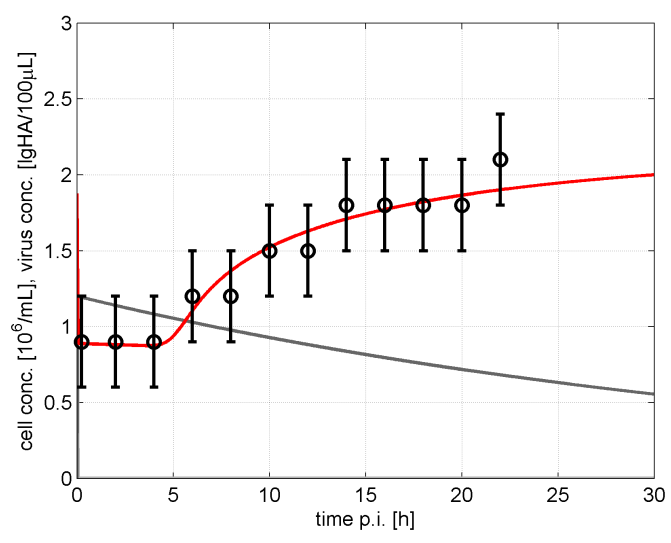

c) $\mathrm{MOI}=3.0$

Figure 13: Comparison of the integral dynamics of the extended model of Müller et al. [2008] with experimental data of Schulze-Horsel et al. [2008] for the infection of adherent MDCK cells with $\mathrm{A} / \mathrm{Equi} / \mathrm{H} 3 \mathrm{~N} 8$ at $\mathrm{MOI}=0.025,1.0$, and 3.0 - lines represent simulation results for the concentration of uninfected cells (light gray), infected cells (sum of cells in and after the latent phase, gray), and virus particles (red); circles represent experimental virus titers of HA measurements including standard error bars of the method $\left( \pm 0.3 \frac{\lg \mathrm{HA}}{100 \mathrm{\mu l}}\right)$. The applied parameter set and initial conditions are summarized in Tab. II (page 28).

\section{Integral dynamics}

Figure 13 shows the comparison of the integral dynamics between simulation and experiment. The model is capable of producing the dynamic courses of virus concentrations, which are in very good agreement with experimental virus titers for different initial conditions.

While the length of the latent period for the experiment with MOI $=0.025$ is rather short 
$(\tau=0.7 \mathrm{~h})$, the virus concentration stays below the detection limit of the HA assay until about $10 \mathrm{~h}$ p.i. because of the relatively low MOI. The courses of the concentrations of uninfected cells and infected cells (sum of cells in and after the latent phase) are smoother when compared with the ones of Fig. 6 (page 16) because the first generation of infected cells already starts to release virus particles less than one hour post infection.

Already at $\mathrm{MOI}=1.0$ all cells are infected instantly by the virus seed and the virus concentration drops below the detection limit of the HA assay. After the latent phase is completed, all cells start to produce virus particles together and the virus concentration rises quickly.

As there are more infectious virus particles than uninfected cells at $\mathrm{MOI}=3.0$, all cells are infected at once, with a considerable number of virus particles left unattached. During the latent phase the virus concentration of the simulation slightly decreases because of virus degeneration. At about $5 \mathrm{~h}$ p.i. the virus titer starts to rise again. As described in Section 3.5 (page 28 ff.), the experiment with MOI = 3.0 deviates from the other two experiments. Presumably, this stems from a considerably lower number of infectious virus particles. The relatively low virus release rate and prolonged duration of the latent period support this assumption. Although, the rather large standard error of the HA-assay leaves room for interpretation.

\section{Distribution dynamics}

In Fig. 14 the mean fluorescence intensities of the simulation are compared with experimental MFIs. For all MOIs the experimental MFI rises quickly after completion of the more or less pronounced latent period. For MOI $=0.025$ and 1.0 a subsequent decline is noticable. Though, due to sparse experimental data the exact course is not known. In the case of MOI $=3.0$ measurements were performed with a higher sampling rate. But, data collection stopped after $22 \mathrm{~h}$ p.i. yielding no clear signs for a potential phase of declining MFI.

In general, the comparison reveals the limitations of the model. While the initial increase of the MFI at least can be reproduced to some extent, a subsequent decrease seems to be unfeasible. It is also striking, especially for MOI $=3.0$, that the delay, which is produced by the latent phase and fits the integral dynamics quite nicely, seems to be too long when the internal dynamics are compared. This could be an indication for the necessity to discriminate between the eclipse phase and the latent phase.

In Figures 15 to 20 (see page 36 ff.) the cell distributions of the simulation are compared to the respective experimental cell distributions, which are determined by Eq. (3.5) (see page 19). The simulated cell distributions depicted in Figures 15 to 20 are 


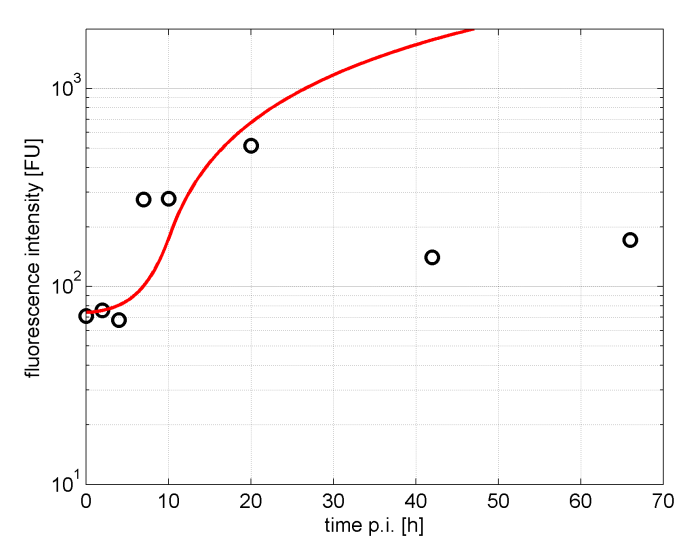

a) $\mathrm{MOI}=0.025$

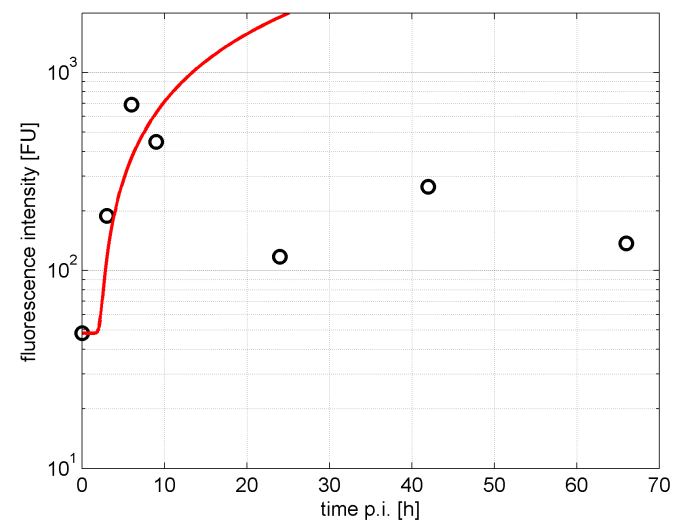

b) $\mathrm{MOI}=1.0$

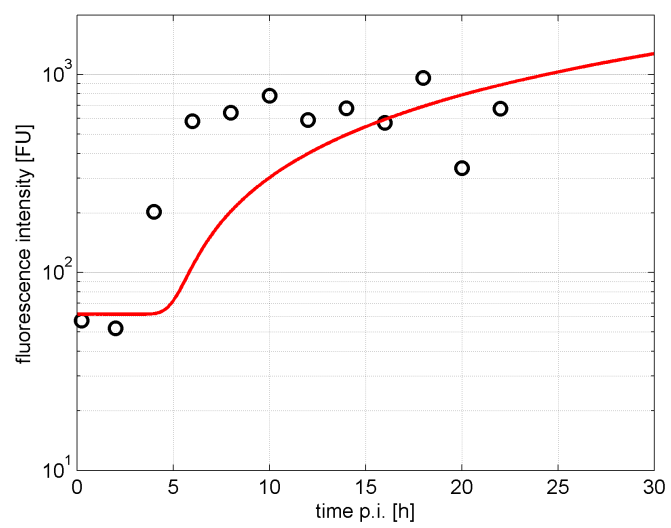

c) $\mathrm{MOI}=3.0$

Figure 14: Comparison of the mean fluorescence intensities (MFIs) of the extended model of Müller et al. [2008] with experimental data of Schulze-Horsel et al. [2008] - simulated MFIs are represented by lines; experimental MFIs are represented by circles.

calculated from the scaled and normalized sum of the individual cell distributions of all cell populations:

$$
\begin{gathered}
q_{\mathrm{c}, i}(t)=\frac{\left(U_{\mathrm{c}}(t)+\Delta \lambda \sum_{j=1}^{M} \hat{L}_{\mathrm{c}, j}(t)\right) q_{\mathrm{sim}, i, 0}+\tilde{I}_{\mathrm{c}, i}(t)}{U_{\mathrm{c}}(t)+\Delta \lambda \sum_{j=1}^{M} \hat{L}_{\mathrm{c}, j}(t)+\sum_{j=1}^{N} \tilde{I}_{\mathrm{c}, j}(t) \Delta \varphi_{j}} \cdot \frac{\Delta \varphi_{i}}{\Delta F_{\mathrm{gg}}} \quad\left[\frac{1}{" \lg \mathrm{FU} "}\right] \\
\text { for } i \in \mathbb{N},[1, N] \quad \text { with } \quad \Delta F_{\mathrm{lg}}=\frac{4}{N} \quad[" \lg \mathrm{FU} "]
\end{gathered}
$$

For MOI $=0.025$ the results are depicted in Fig. 15 and 16. As can already be derived from the MFI, the simulation lags behind at $7 \mathrm{~h}$ p.i., but is ahead from $20 \mathrm{~h}$ p.i. on. The 


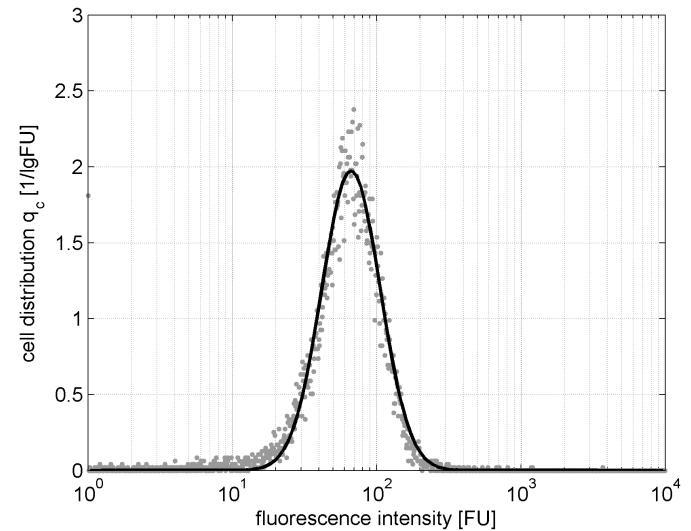

a) $t=0 \mathrm{~h}$ p.i.

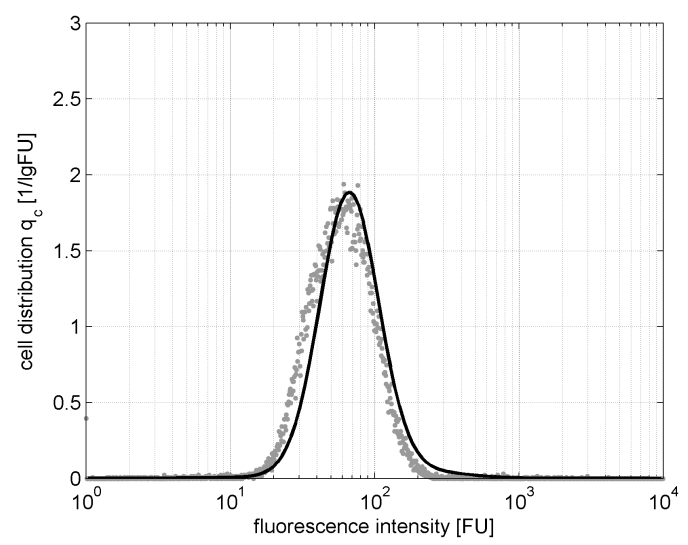

c) $t=4$ h p.i.

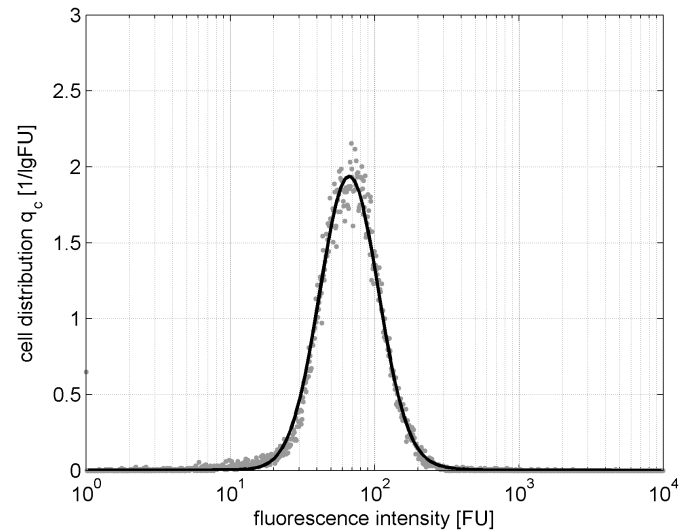

b) $t=2 \mathrm{~h}$ p.i.

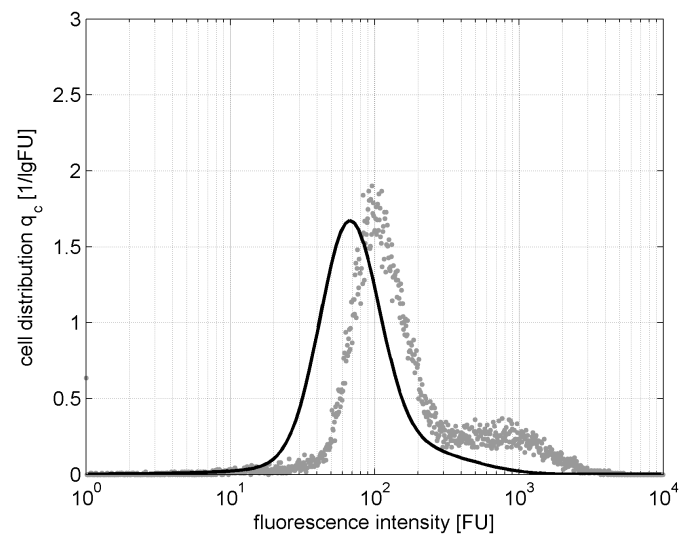

d) $t=7 \mathrm{~h} \mathrm{p.i.}$

Figure 15: Comparison of the distribution dynamics of the extended model of Müller et al. [2008] with experimental data of Schulze-Horsel et al. [2008] at MOI =0.025 (Part I) - simulated cell distributions are represented by lines; experimental cell distributions are represented by dots.

experimental cell distributions at 7 and $10 \mathrm{~h}$ p.i. exhibit a stepped course at higher fluorescence intensities. These are the first infected cells which have completed the lag phase and start to produce viral protein. Thus, the fluorescence intensity of the cells increases and they leave the initial peak behind. In the simulations this process is indicated by only a slight bend in the slope of the distribution because $\tau$ is rather small. For larger values of $\tau$ the effect would be more obvious.

For MOI $=1.0$ and 3.0 this phenomenon of part of the cell population rushing ahead of the rest is even more pronounced (see Figures 17 and 19). At $3 \mathrm{~h}$ p.i. (MOI = 1.0) and at $4 \mathrm{~h}$ p.i. $(\mathrm{MOI}=3.0)$ the experimental cell distributions clearly show bimodality, which is not reproduced by the model, because in the simulation all cells are infected virtually 


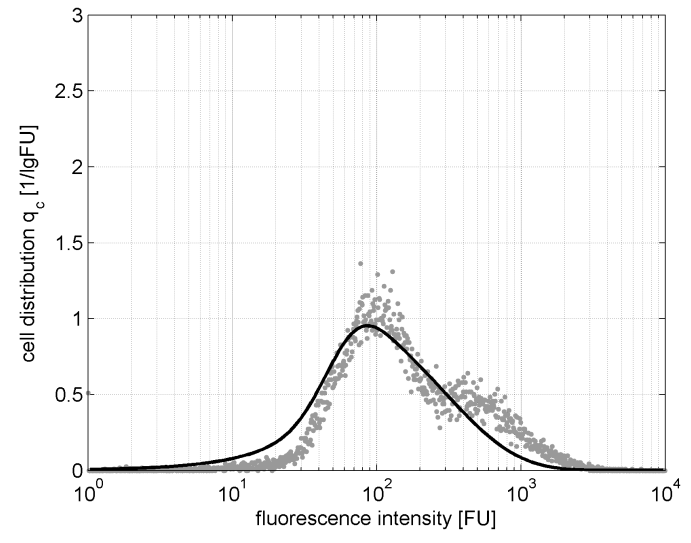

a) $t=10 \mathrm{~h}$ p.i.

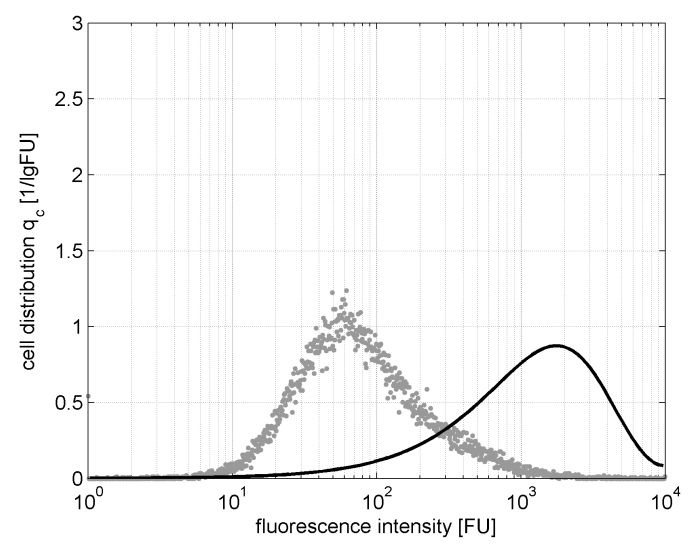

c) $t=42 \mathrm{~h}$ p.i.

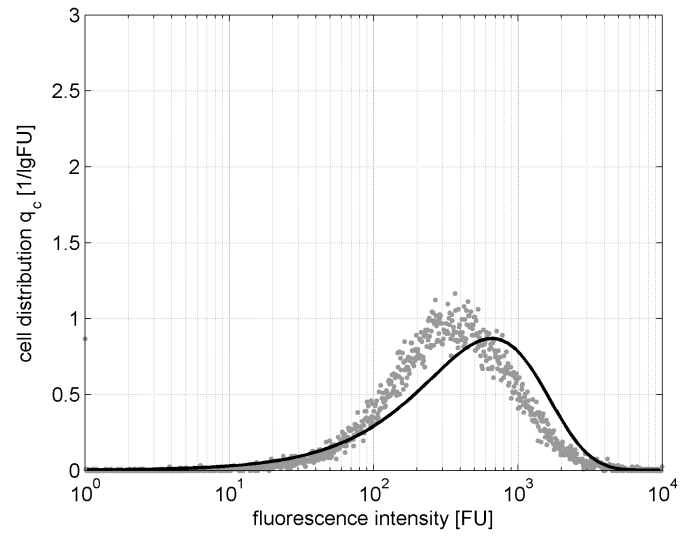

b) $t=20 \mathrm{~h} \mathrm{p.i.}$

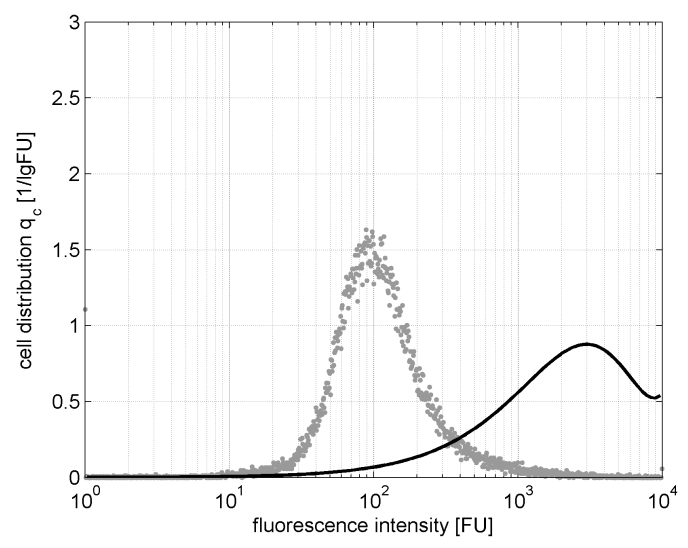

d) $t=66 \mathrm{~h}$ p.i.

Figure 16: Comparison of the distribution dynamics of the extended model of Müller et al. [2008] with experimental data of Schulze-Horsel et al. [2008] at MOI $=0.025$ (Part II) - simulated cell distributions are represented by lines; experimental cell distributions are represented by dots.

at the same time at these MOIs. When looking at the experimental cell distributions this seems not to be the case. At MOI $=1.0$ approximately half of the cell population is infected and produces viral protein at $3 \mathrm{~h}$ p.i. Surprisingly, at MOI $=3.0$ not many more cells are producing viral protein at $4 \mathrm{~h}$ p.i. This can be attributed to the less than expected amount of infectious virus particles in the virus seed for the respective experiment. If one takes the ratio between the coefficients for infection and virus attachment as a measure for the achieved plating efficiency, the actual MOI turns out to be (see Eq. (3.26) and Tab. II on page $28 \mathrm{f}$.)

$$
\mathrm{MOI}=\frac{V_{0} P_{\mathrm{eff}}}{U_{\mathrm{c}, 0}}=\frac{V_{0}}{U_{\mathrm{c}, 0}} \cdot \frac{k_{\mathrm{vi}}}{k_{\mathrm{va}}}=\frac{1.507 \cdot 10^{9}}{1.2 \cdot 10^{6}} \cdot \frac{0.71 \cdot 10^{-3}}{0.8}=1.115
$$




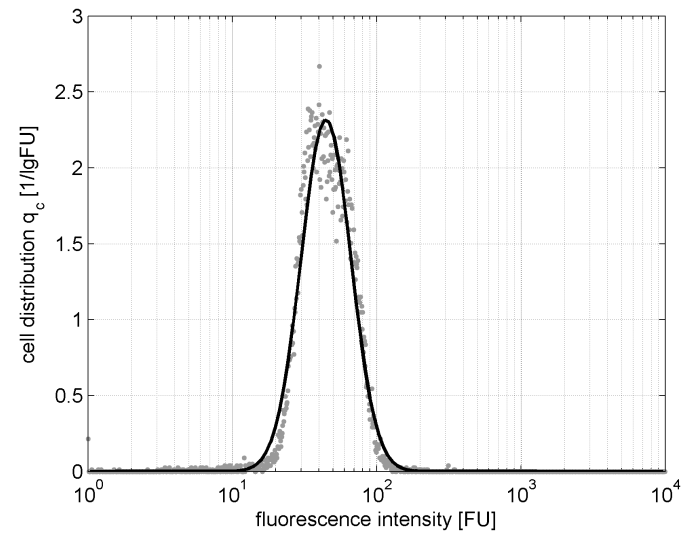

a) $t=0 \mathrm{~h}$ p.i.

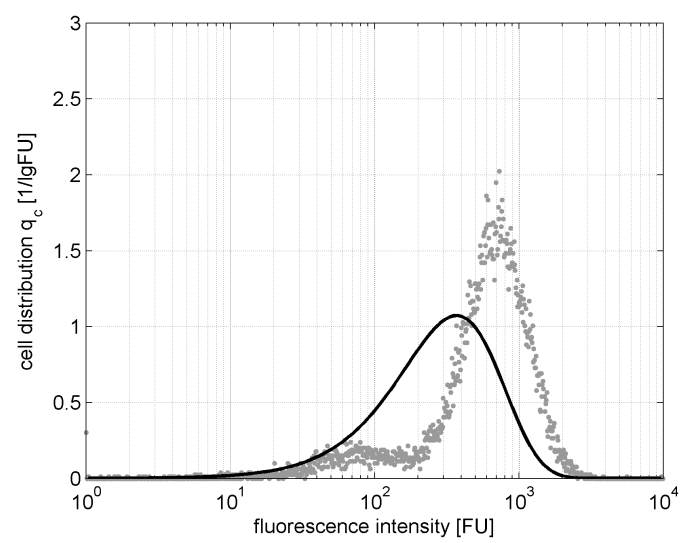

c) $t=6 \mathrm{~h}$ p.i.

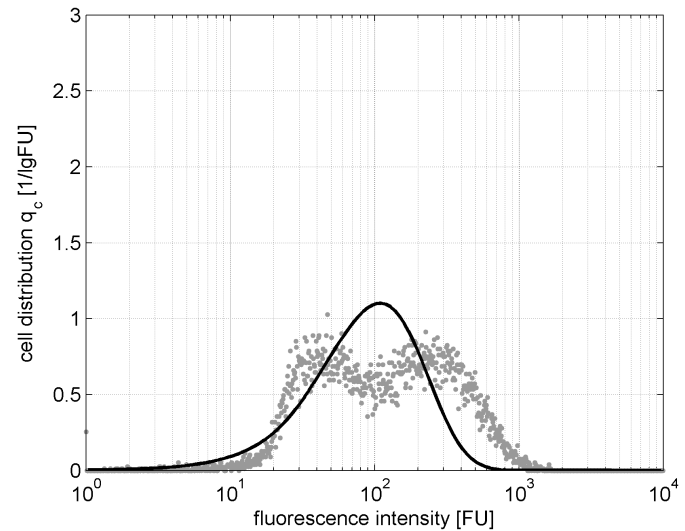

b) $t=3 \mathrm{~h}$ p.i.

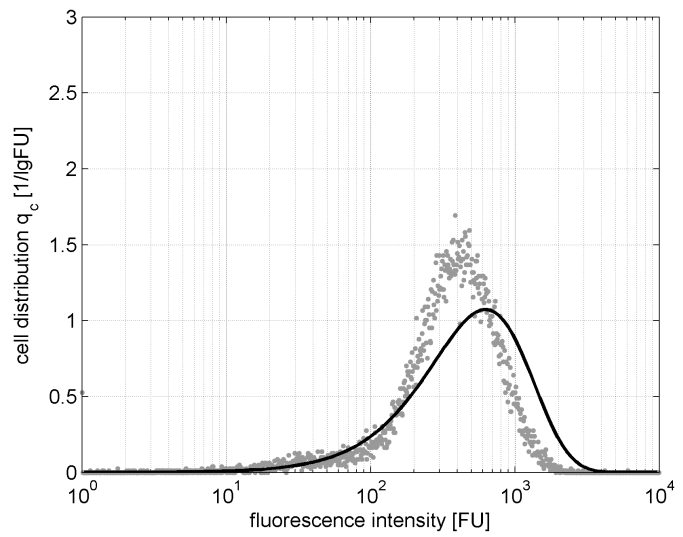

d) $t=9 \mathrm{~h} \mathrm{p.i.}$

Figure 17: Comparison of the distribution dynamics of the extended model of Müller et al. [2008] with experimental data of Schulze-Horsel et al. [2008] at MOI = 1.0 (Part I) - simulated cell distributions are represented by lines; experimental cell distributions are represented by dots.

Hence, both experiments (MOI = 1.0 and "3.0") are not too far away from each other with regard to the distribution dynamics. However, an increased amount of DIPs supposed for the experiment with MOI $=3.0$ - may presumably be the reason for the deviation of the integral behavior.

Furthermore, the experimental cell distributions of MOI $=1.0$ and 3.0 exhibit tailing after the bimodality is dissolved, which is at least partly reproduced by the simulation. The extent of tailing in the experiments suggests that a small fraction of the cell population is either still uninfected, stuck in the latent phase, or very poor in producing viral protein. The last two options could be a hint to a large variability in the ability to 
produce viral protein or to the existence of an antiviral state.

As mentioned above, the reversal of the propagation direction, as observed for later time points at MOI $=0.025$ and 1.0 , can not be accomplished by the presented model. Even if the virus protein production coefficient $k_{\text {pro }}$ was made dependent on $\varphi$, any combination of $k_{\text {pro }}(\varphi)$ and the virus release coefficient $k_{\text {rel }}$ would only result in a movement of the cell distribution either to higher degrees of fluorescence or to lower degrees of fluorescence depending on whether the difference between both coefficients $\left(k_{\mathrm{pro}}(\varphi)-k_{\text {rel }}\right)$ is positive or negative. Furthermore, whenever this difference equals zero a stagnation point would be created, which could not be crossed.

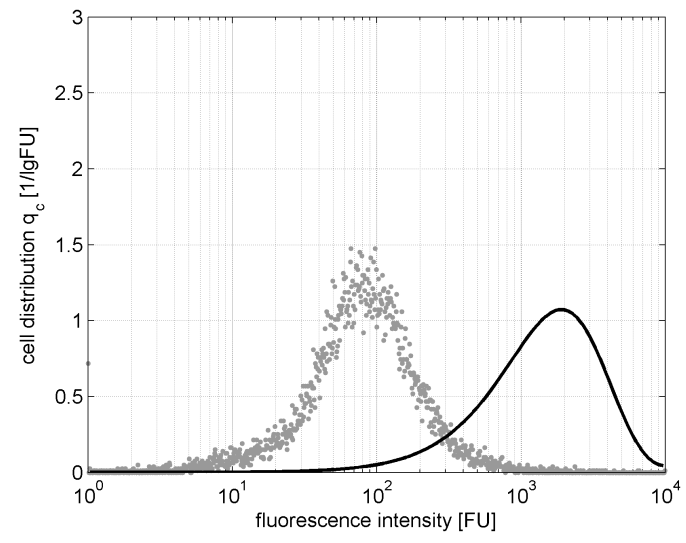

a) $t=24 \mathrm{~h}$ p.i.

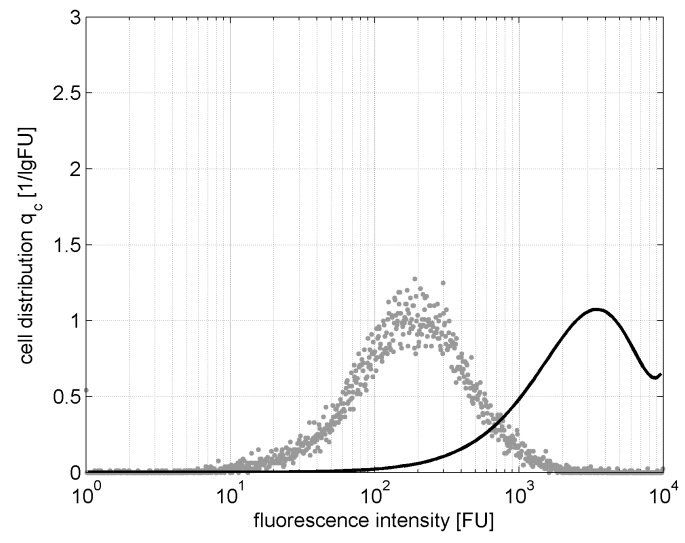

b) $t=42 \mathrm{~h}$ p.i.

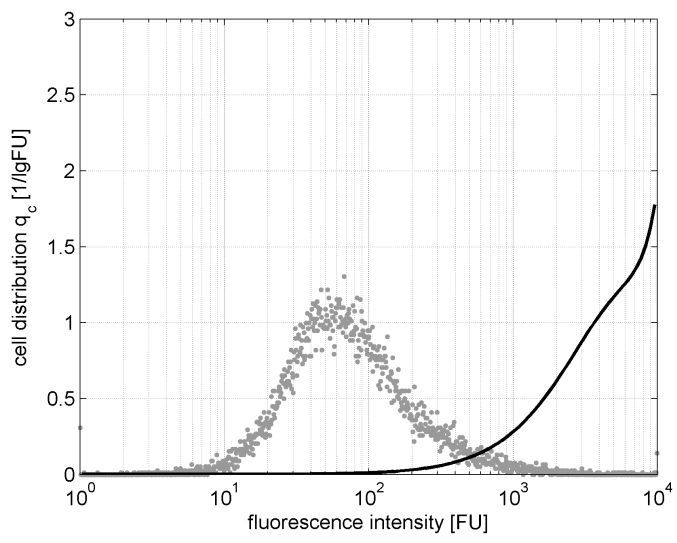

c) $t=66 \mathrm{~h}$ p.i.

Figure 18: Comparison of the distribution dynamics of the extended model of Müller et al. [2008] with experimental data of Schulze-Horsel et al. [2008] at MOI = 1.0 (Part II) - simulated cell distributions are represented by lines; experimental cell distributions are represented by dots. 
At the time the model was created, of all the issues which needed to be solved on the way to model the dynamic features of the process, finding a structure which would allow the model to reproduce the reversal of the propagation direction was given the highest priority. Hence, the path for the next steps was layed. 


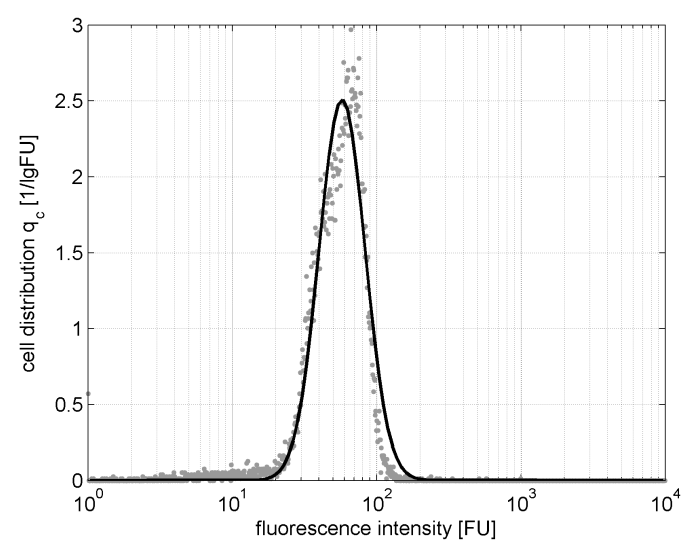

a) $t=0.25 \mathrm{~h} \mathrm{p.i.}$

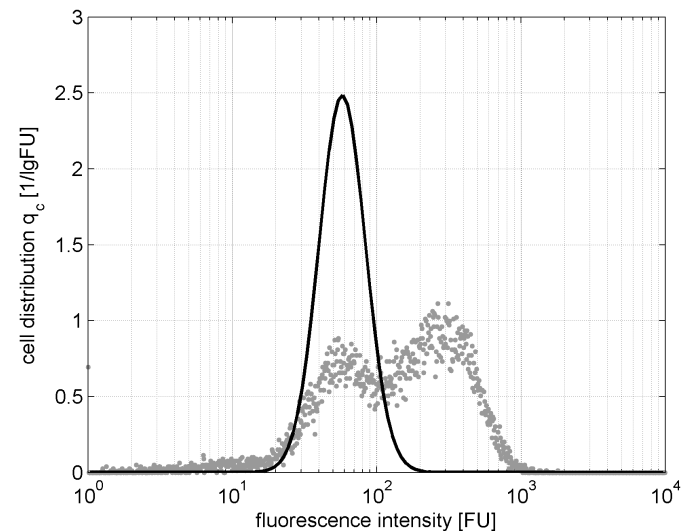

c) $t=4 \mathrm{~h}$ p.i.

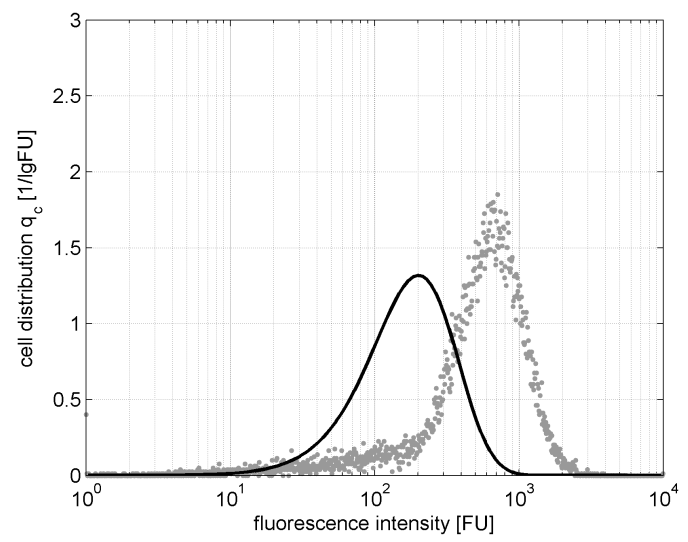

e) $t=8 \mathrm{~h}$ p.i.

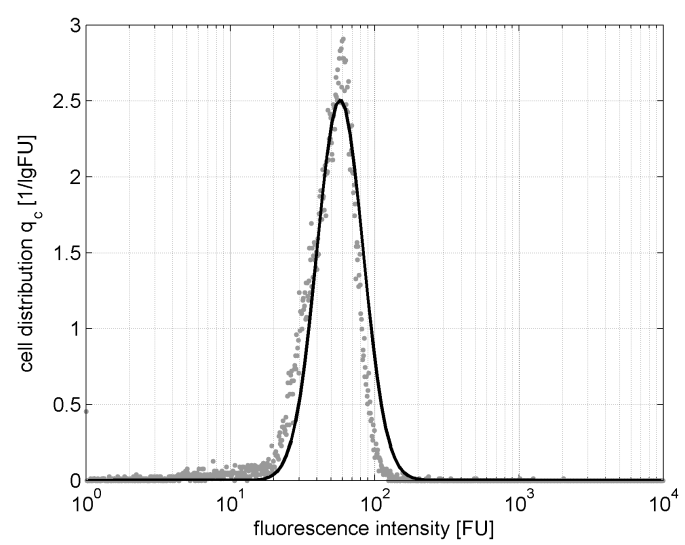

b) $t=2 \mathrm{~h}$ p.i.

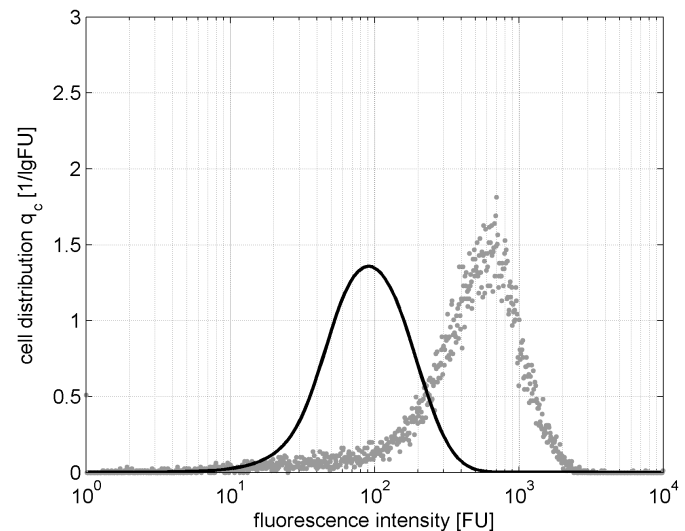

d) $t=6 \mathrm{~h}$ p.i.

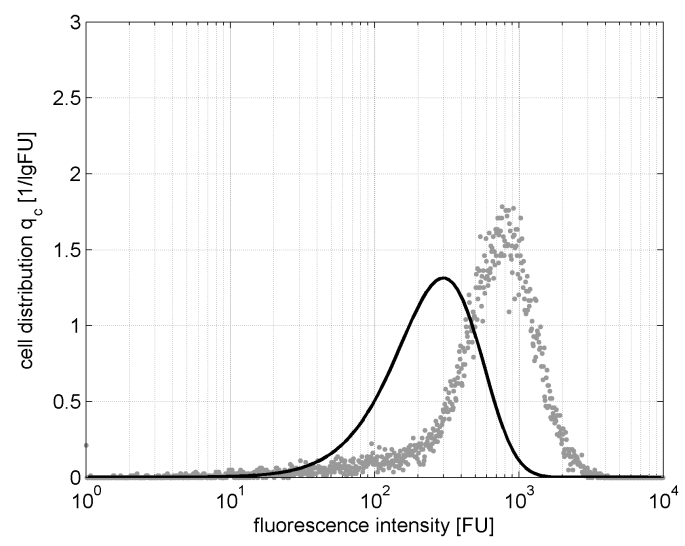

f) $t=10 \mathrm{~h}$ p.i.

Figure 19: Comparison of the distribution dynamics of the extended model of Müller et al. [2008] with experimental data of Schulze-Horsel et al. [2008] at MOI $=3.0$ (Part I) - simulated cell distributions are represented by lines; experimental cell distributions are represented by dots. 


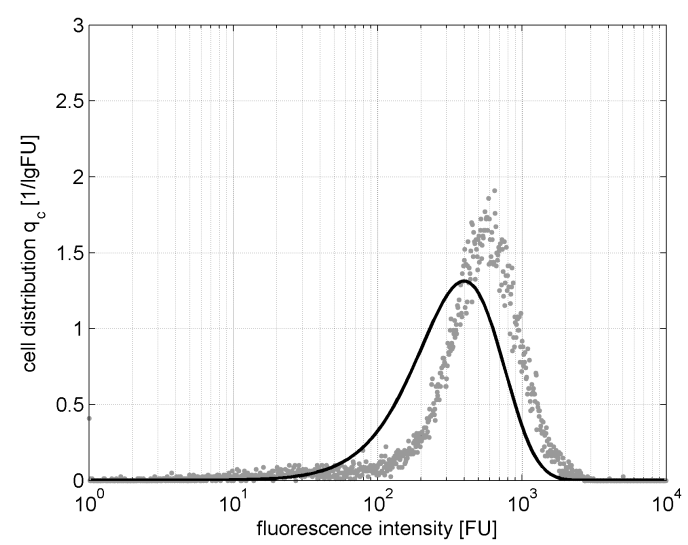

a) $t=12 \mathrm{~h}$ p.i.

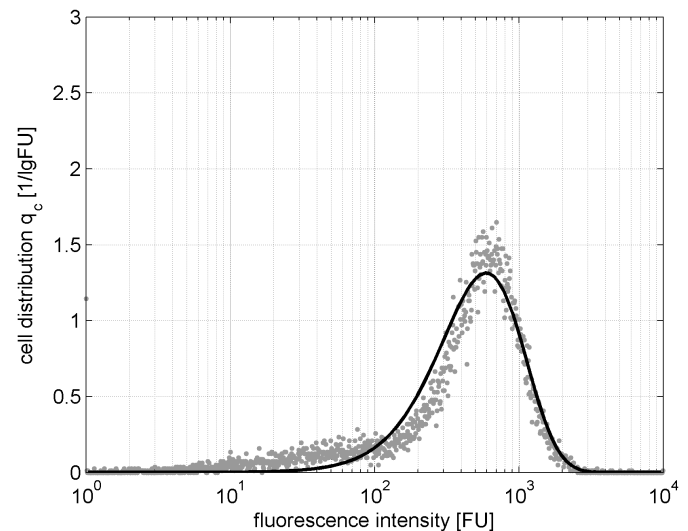

c) $t=16 \mathrm{~h}$ p.i.

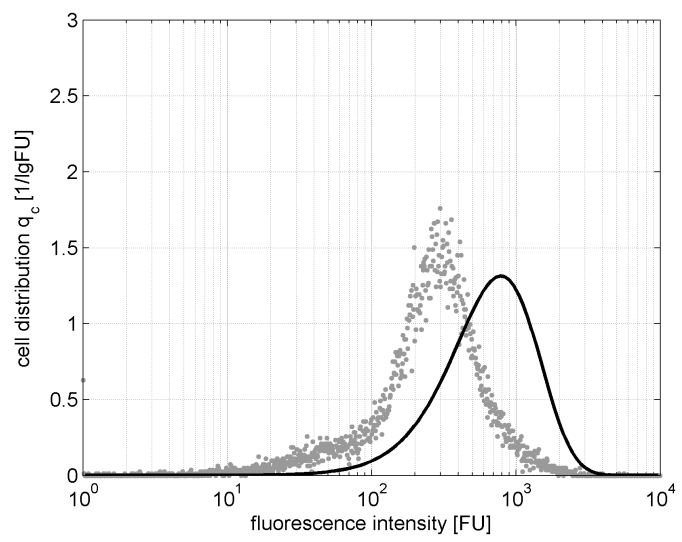

e) $t=20 \mathrm{hp}$.i.

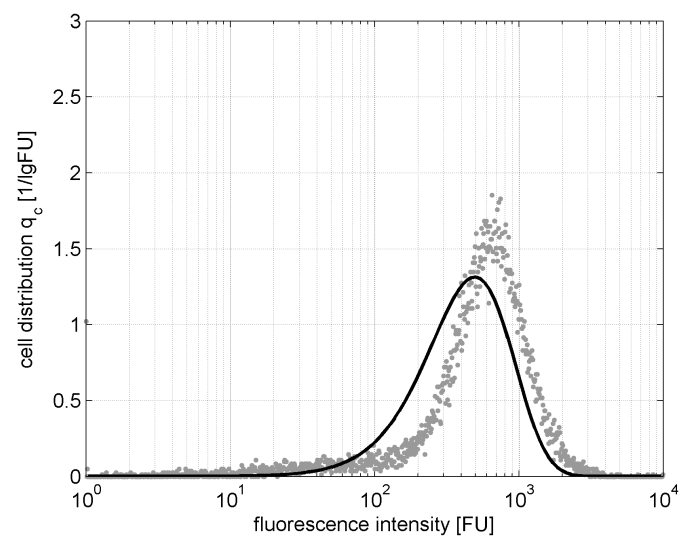

b) $t=14 \mathrm{~h}$ p.i.

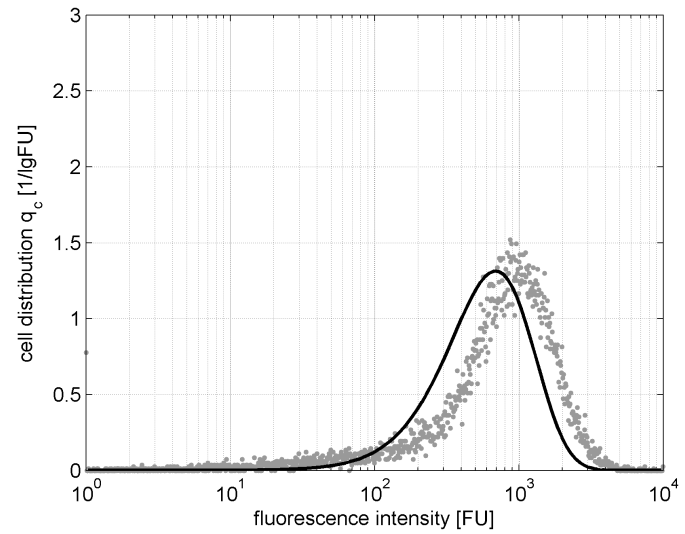

d) $t=18 \mathrm{~h}$ p.i.

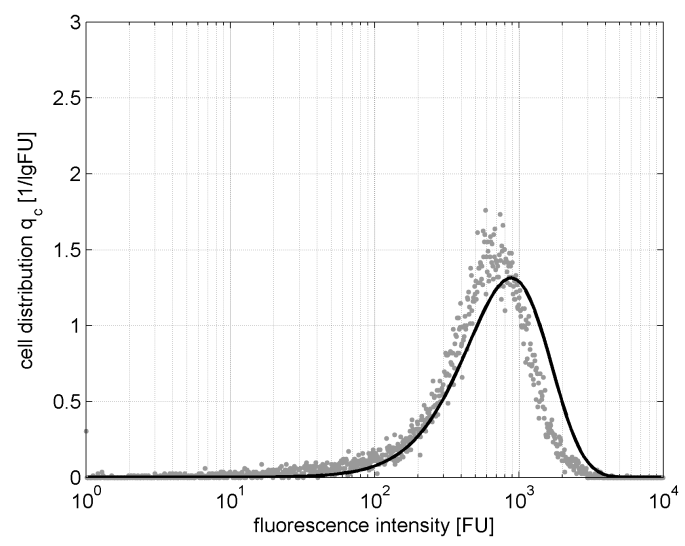

f) $t=22 \mathrm{~h}$ p.i.

Figure 20: Comparison of the distribution dynamics of the extended model of Müller et al. [2008] with experimental data of Schulze-Horsel et al. [2008] at MOI = 3.0 (Part II) - simulated cell distributions are represented by lines; experimental cell distributions are represented by dots. 


\section{Implementation of the backshift}

In order to model the reversal of the propagation direction of the cell distribution, the so called backshift, the viral protein production process is assumed to be divided into two phases. While the virus protein production exceeds the opposing process of virus release in the first phase, this relation is reversed in the second phase. Hence, the degree of fluorescence increases during the first phase and decreases during the second phase. In the resulting "two-phase model" the species of virus protein producing infected cells is divided into two species, corresponding to the two phases of viral protein production $\left(\tilde{I}_{\mathrm{c}}^{\mathrm{I}}\right.$ and $\left.\tilde{I}_{\mathrm{c}}^{\mathrm{II}}\right)$. Except from the viral protein production the two species of infected cells are assumed to behave exactly the same.

\subsection{Model equations}

The mathematical descriptions of the uninfected cells and the infected cells in the latent phase stay untouched (see Eqs. (3.9) and (3.12), page 22 f.). After the latent period, infected cells start to produce viral protein in production phase I with the corresponding coefficient $k_{\text {pro }}^{\mathrm{I}}$. With regard to the release of virus particles and the death due to viral infection nothing changes in comparison to Eq. (3.13) (page 23). The transition from one species or phase to the other is controlled by the phase transition coefficient $k_{\mathrm{pt}}$, which depends on the degree of fluorescence,

$$
\begin{aligned}
\frac{\partial \tilde{I}_{\mathrm{c}}^{\mathrm{I}}(t, \varphi)}{\partial t}= & k_{\mathrm{vi}} \tilde{U}_{\mathrm{c}}(t-\tau, \varphi) V(t-\tau) \cdot \mathrm{e}^{-k_{\mathrm{cdv}} \tau}-\left(k_{\mathrm{pro}}^{\mathrm{I}}-k_{\mathrm{rel}}\right) \frac{\partial \tilde{I}_{\mathrm{c}}^{\mathrm{I}}(t, \varphi)}{\partial \varphi} \\
& -k_{\mathrm{cdv}} \tilde{I}_{\mathrm{c}}^{\mathrm{I}}(t, \varphi)-k_{\mathrm{pt}}(\varphi) \tilde{I}_{\mathrm{c}}^{\mathrm{I}}(t, \varphi) .
\end{aligned}
$$

The cells in production phase II emerge from cells in production phase I, from which they differ in terms of viral protein production. This is taken into account by the corresponding production coefficient $k_{\text {pro }}^{\mathrm{II}}$, which is a function of $\varphi$,

$$
\frac{\partial \tilde{I}_{\mathrm{c}}^{\mathrm{II}}(t, \varphi)}{\partial t}=k_{\mathrm{pt}}(\varphi) \tilde{I}_{\mathrm{c}}^{\mathrm{I}}(t, \varphi)-\frac{\partial}{\partial \varphi}\left(k_{\mathrm{pro}}^{\mathrm{II}}(\varphi)-k_{\mathrm{rel}}\right) \tilde{I}_{\mathrm{c}}^{\mathrm{II}}(t, \varphi)-k_{\mathrm{cdv}} \tilde{I}_{\mathrm{c}}^{\mathrm{II}}(t, \varphi) .
$$

With respect to the changed model formulation the virus balance equation and the cell growth coefficient have to be reconsidered:

$$
\frac{\mathrm{d} V(t)}{\mathrm{d} t}=k_{\mathrm{rel}} \int_{\varphi}\left(\tilde{I}_{\mathrm{c}}^{\mathrm{I}}(t, \varphi)+\tilde{I}_{\mathrm{c}}^{\mathrm{II}}(t, \varphi)\right) \mathrm{d} \varphi-k_{\mathrm{vd}} V(t)-k_{\mathrm{va}} V(t) \int_{\varphi} \tilde{U}_{\mathrm{c}}(t, \varphi) \mathrm{d} \varphi,
$$




$$
\mu_{\mathrm{c}}(t)=\mu_{\mathrm{c}, \max } \frac{C_{\max }-\int_{\varphi}\left(\tilde{U}_{\mathrm{c}}(t, \varphi)+\tilde{L}_{\mathrm{c}}(t, \varphi)+\tilde{I}_{\mathrm{c}}^{\mathrm{I}}(t, \varphi)+\tilde{I}_{\mathrm{c}}^{\mathrm{II}}(t, \varphi)\right) \mathrm{d} \varphi}{C_{\max }} .
$$

\subsection{Parameters}

As this "two-phase model" is used only as a conceptual study, no rigorous parameter fitting was performed. Therefore, nearly all parameters that already existed in the extended model of Müller et al. [2008] were kept as they are. Only $k_{\text {pro }}^{\mathrm{I}}$, which has the same significance as $k_{\text {pro }}$, was refitted.

By testing various definitions and combinations of the phase transition coefficient $\left(k_{\mathrm{pt}}\right)$ and the virus protein production coefficient in phase II $\left(k_{\text {pro }}^{\mathrm{II}}\right)$ it turned out, that the functional dependency on $\varphi$ of both parameters is essential for the description of the backshift.

For $k_{\mathrm{pt}}(\varphi)$ a normalized cumulative distribution function is chosen

$$
\begin{gathered}
k_{\mathrm{pt}}(\varphi)=\frac{\int_{\min }^{\varphi} \exp \left[-\frac{1}{2}\left(\frac{\varphi F_{\mathrm{VE}}-\mu_{\mathrm{pt}}}{\sigma_{\mathrm{pt}}}\right)^{2}\right] \mathrm{d} \varphi}{\int_{\varphi_{\min }} \exp \left[-\frac{1}{2}\left(\frac{\varphi F_{\mathrm{VE}}-\mu_{\mathrm{pt}}}{\sigma_{\mathrm{pt}}}\right)^{2}\right] \mathrm{d} \varphi}\left(k_{\mathrm{pt}, \max }-k_{\mathrm{pt}, \text { min }}\right)+k_{\mathrm{pt}, \min } \\
\text { with } \varphi_{\min }=1 \mathrm{FU} / F_{\mathrm{VE}} \text { and } \varphi_{\max }=10^{4} \mathrm{FU} / F_{\mathrm{VE}} .
\end{gathered}
$$

Accordingly, $k_{\mathrm{pt}}(\varphi)$ runs from $k_{\mathrm{pt} \text {,min }}$ for low values of $\varphi$ to $k_{\mathrm{pt} \text {,max }}$ for high values of $\varphi$ with an expectation of $\mu_{\mathrm{pt}}$ and a standard deviation of $\sigma_{\mathrm{pt}}$. In this way, a smooth phase transition can be achieved, which is minimal (zero) for low degrees of fluorescence and maximal for high degrees of fluorescence. Thus, the more intracellular viral protein is present the higher the chance for a transition to phase II.

For $k_{\text {pro }}^{\mathrm{II}}(\varphi)$ a linear dependency on $\varphi$ is assumed

$$
k_{\text {pro }}^{\mathrm{II}}(\varphi)=\frac{k_{\text {pro }}^{\mathrm{II}}\left(\varphi_{\max }\right)-k_{\mathrm{pro}}^{\mathrm{II}}\left(\varphi_{\min }\right)}{\varphi_{\max }-\varphi_{\min }}\left(\varphi-\varphi_{\min }\right)+k_{\text {pro }}^{\mathrm{II}}\left(\varphi_{\min }\right) .
$$

Hence, $k_{\text {pro }}^{\mathrm{II}}(\varphi)$ runs linearly from $k_{\text {pro }}^{\mathrm{II}}\left(\varphi_{\min }\right)$ to $k_{\text {pro }}^{\mathrm{II}}\left(\varphi_{\max }\right)$. As the degree of fluorescence is generally decreased during phase II the viral protein production rate has to be lower than the virus release rate. For high degrees of fluorescence the difference between $k_{\text {pro }}^{\mathrm{II}}$ and $k_{\text {rel }}$ has to be rather large to allow for a fast backshift of the cell population. On the other hand, the decrease in $\varphi$ has to slow down at lower degrees of fluorescence to avoid 


\begin{tabular}{rcl}
\hline Parameter & Value & Unit \\
\hline$C_{\text {max }}$ & $1.2 \cdot 10^{6}$ & $\mathrm{ml}^{-1}$ \\
$k_{\mathrm{cdf}}$ & 0.001 & $\mathrm{~h}^{-1}$ \\
$k_{\mathrm{cdv}}$ & $25.7 \cdot 10^{-3}$ & $\mathrm{~h}^{-1}$ \\
$k_{\mathrm{pro}}^{\mathrm{I}}$ & 255 & $\mathrm{~h}^{-1}$ \\
$k_{\text {pro }}^{\mathrm{II}}\left(\varphi_{\min }\right)$ & 246 & $\mathrm{~h}^{-1}$ \\
$k_{\mathrm{pro}}^{\mathrm{II}}\left(\varphi_{\max }\right)$ & 126 & $\mathrm{~h}^{-1}$ \\
$k_{\mathrm{pt}, \min }$ & 0 & $\mathrm{~h}^{-1}$ \\
$k_{\mathrm{pt}, \max }$ & 0.8 & $\mathrm{~h}^{-1}$ \\
$k_{\mathrm{rel}}$ & 246 & $\mathrm{~h}^{-1}$ \\
$k_{\mathrm{va}}$ & 0.8 & $\mathrm{ml} / \mathrm{h}$ \\
$k_{\mathrm{vd}}$ & 0.009 & $\mathrm{~h}^{-1}$ \\
$k_{\mathrm{vi}}$ & $1.9 \cdot 10^{-3}$ & $\mathrm{ml} / \mathrm{h}$ \\
$\mu_{\mathrm{c}, \max }$ & 0.03 & $\mathrm{~h}^{-1}$ \\
$\mu_{\mathrm{pt}}$ & 800 & $\mathrm{FU}$ \\
$\sigma_{\mathrm{pt}}$ & 800 & $\mathrm{FU}$ \\
$\tau$ & 0.7 & $\mathrm{~h}$ \\
\hline$I_{\mathrm{c}, 0}$ & 0 & $\mathrm{ml}^{-1}$ \\
$U_{\mathrm{c}, 0}$ & $1.2 \cdot 10^{6}$ & $\mathrm{ml}$ \\
$V_{0}$ & $12.6 \cdot 10^{6}$ & $\mathrm{ml}$ \\
$\mu_{0}$ & 66.5 & $\mathrm{FU}$ \\
$\sigma_{0}$ & 0.202 & $\mathrm{FU}$ \\
\hline & & \\
\hline
\end{tabular}

Table III: Parameter set and initial conditions for the "two-phase model" at MOI $=0.025$. Parameters that are not adopted from Tab. II are marked in gray.

running into the lower boundary. Therefore, the difference between $k_{\text {pro }}^{\mathrm{II}}$ and $k_{\text {rel }}$ has to be minimal for low degrees of fluorescence.

Technically this means that after the latent period cells accumulate viral protein as long as they are in phase I. When the transition to phase II is performed, the amount of intracellular virus protein decreases due to a reduced production rate. But, while the cells are in phase II the depletion of viral protein slows down because of a recovery of the virus protein production rate $\left(k_{\text {pro }}^{\mathrm{II}}\right.$ increases with decreasing $\left.\varphi\right)$. This recovery of $k_{\text {pro }}^{\mathrm{II}}$ seems to be biologically implausible. As a remedy, one could assume that the production rate is lowered below the release rate and stays constant in phase II. Consequently, $k_{\text {rel }}$ has to change in phase II in order to decelerate the increase of $\varphi$, which would, of course, effect the integral dynamics.

Recent publications by Arranz et al. [2012] and Moeller et al. [2012] as well as running 
investigations by the Bioprocess Engineering Group at the MPI Magdeburg lead to another conclusion. Instead of the virus release, at least for viral NP, the formation of viral ribonucleoprotein complexes (RNP) seems to be responsible for the decrease in fluorescence intensity by preventing fluorescein-labeled antibodies from binding to NP. However, the consequences of these findings are being discussed in more detail in the next chapter. For the time being, the model structure presented above is taken as valid. In Tab. III the applied parameter set for MOI $=0.025$ is shown.

\subsection{Simulation results}

The integral dynamics of the "two-phase model" correspond to the ones presented in Section 3.6 (page $32 \mathrm{ff}$.). Even with an unoptimized parameter set the improvement of the distribution dynamics in comparison to the extended model of Müller et al. [2008] becomes apparent. In Fig. 21 the course of the MFI is exemplified for MOI $=0.025$. The backshift is clearly visible. This is underlined by the comparison of simulated and experimental cell distributions of the same MOI in Figs. 22 and 23.

In summary, the proposed model structure constitutes a promising candidate capable of reproducing the reversal of propagation direction of the cell distribution. Although there certainly is room for improvements, the lack of temporally more dense experimental data reduced the benefit of more extensive investigations at the time of development. Further refinement of the model would require an extension of the experimental database to assure a biologically reasonable proceeding. Eventually, new experimental data became available through the work of Schulze-Horsel et al. [2009], in which the production of human influenza virus vaccine in adherent MDCK cells was investigated to a greater extent. The obtained findings and their implications in terms of population balance modeling are presented in the next chapter. 


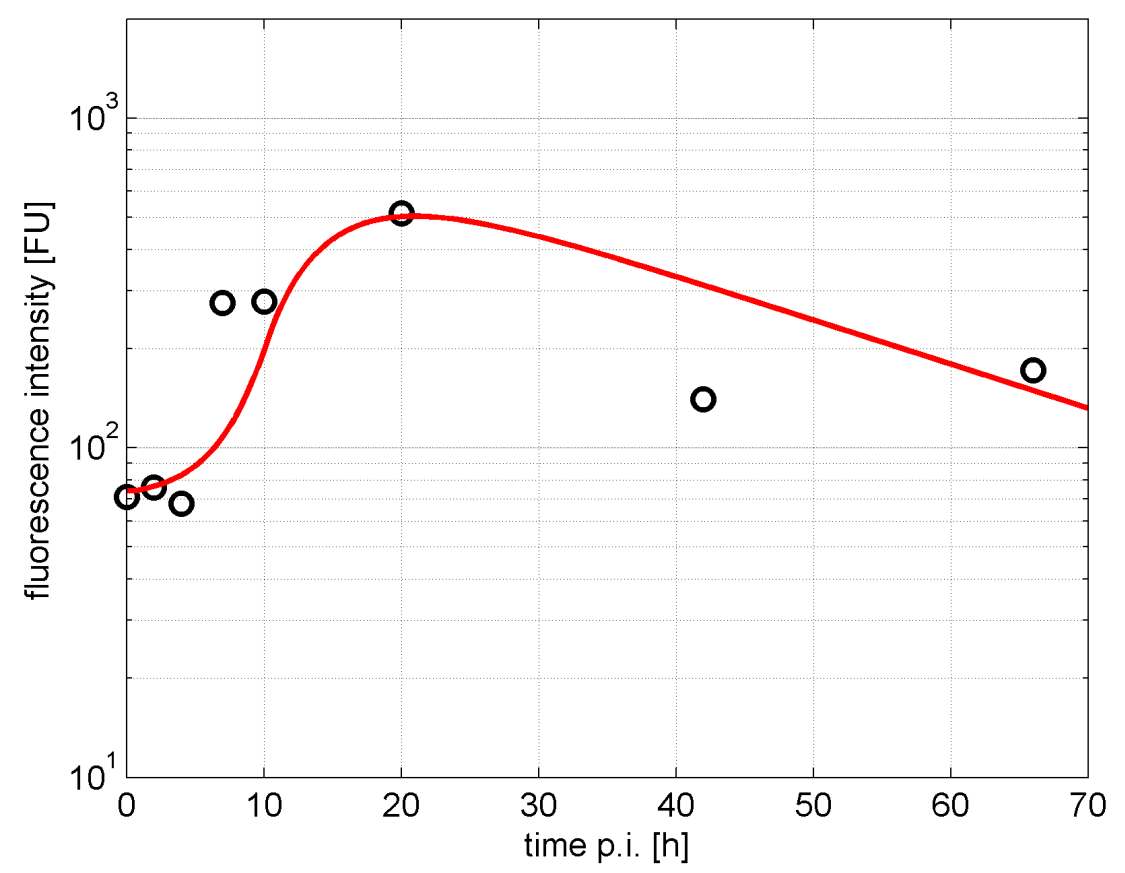

Figure 21: Comparison of the mean fluorescence intensity (MFI) of the "two-phase model" with experimental data of Schulze-Horsel et al. [2008] at MOI $=0.025$ - a solid line represents the temporal change of the simulated MFI; experimental MFIs are represented by circles. 


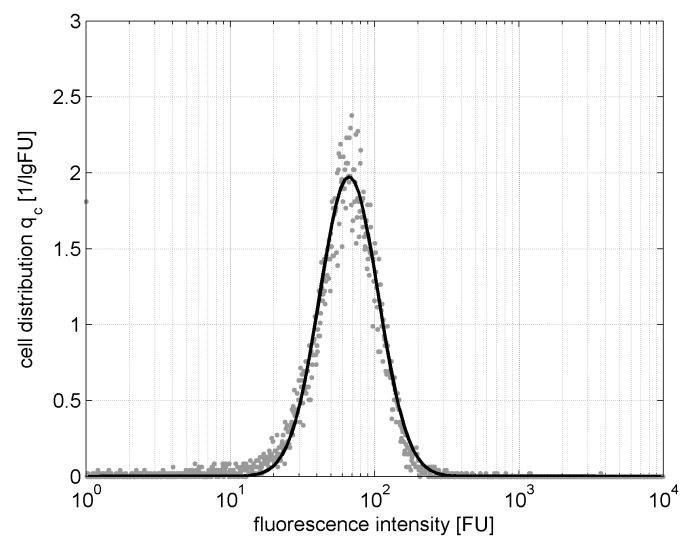

a) $t=0 \mathrm{hp}$.i.

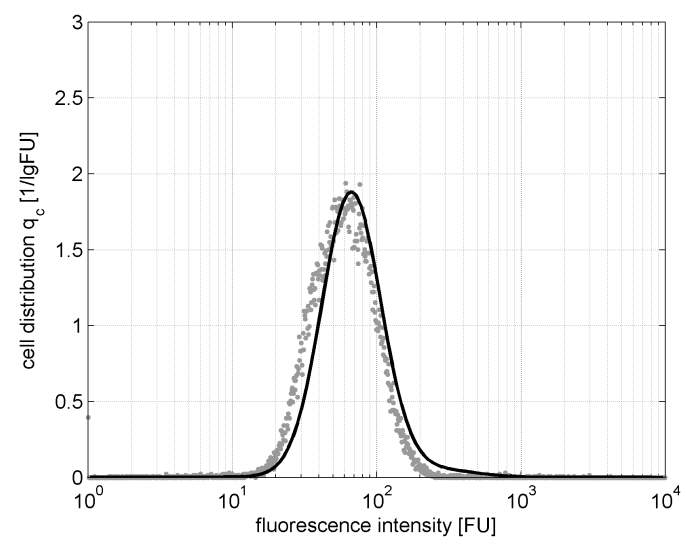

c) $t=4 \mathrm{~h}$ p.i.

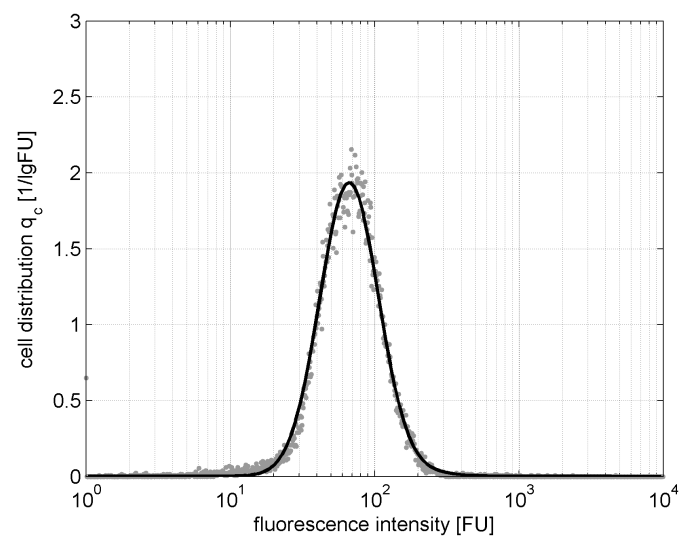

b) $t=2 \mathrm{~h}$ p.i.

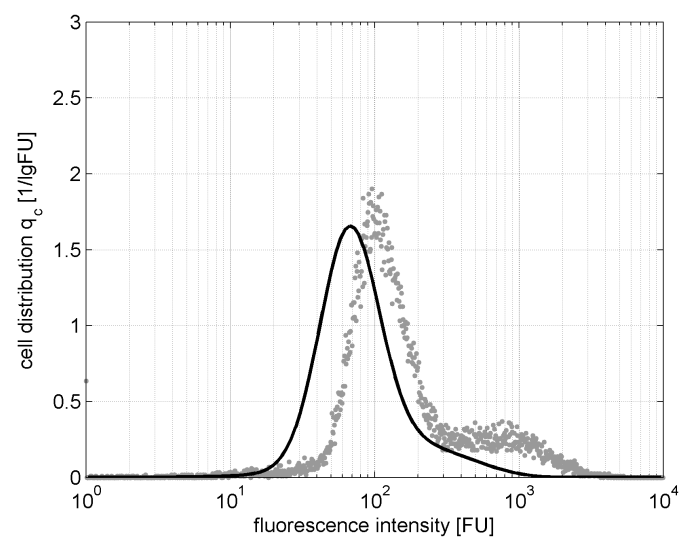

d) $t=7 \mathrm{~h}$ p.i.

Figure 22: Comparison of the distribution dynamics of the "two-phase model" with experimental data of Schulze-Horsel et al. [2008] at MOI $=0.025$ (Part I) - simulated cell distributions are represented by lines; experimental cell distributions are represented by dots. 


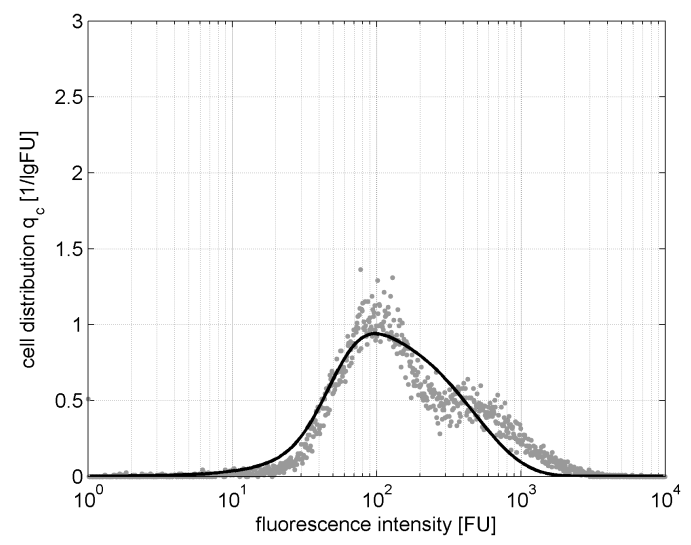

a) $t=10 \mathrm{~h}$ p.i.

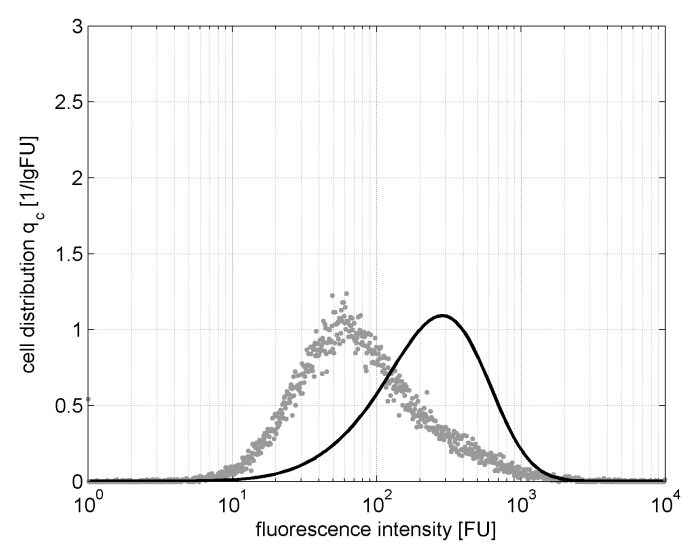

c) $t=42 \mathrm{~h}$ p.i.

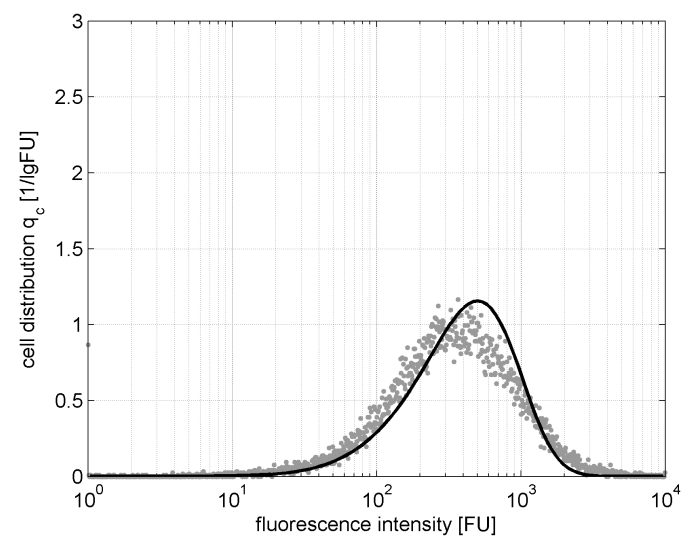

b) $t=20 \mathrm{~h}$ p.i.

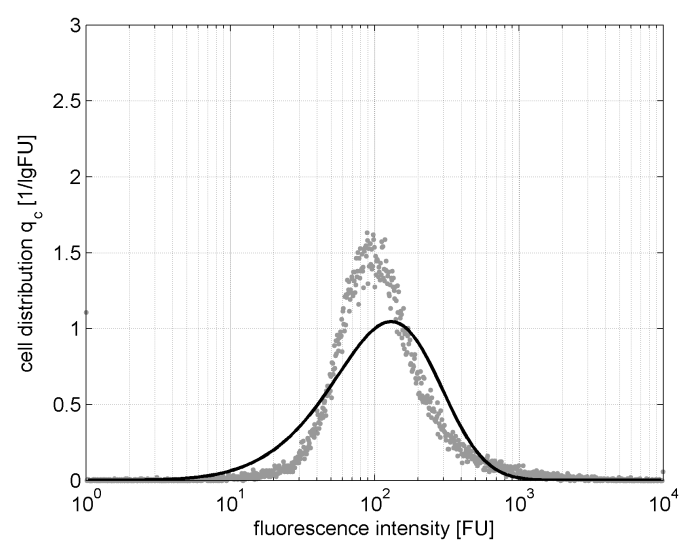

d) $t=66 \mathrm{~h}$ p.i.

Figure 23: Comparison of the distribution dynamics of the "two-phase model" with experimental data of Schulze-Horsel et al. [2008] at MOI $=0.025$ (Part II) - simulated cell distributions are represented by lines; experimental cell distributions are represented by dots. 


\section{Distributed modeling of human influenza A virus replication}

The previously introduced "two-phase model" represents an important intermediate step in the development of what would become the model of Müller et al. [2013]. The fact that the former actually was never officially published does not diminish its significance as the concept of a multi-phase virus replication turned out to be useful in the end. Though, the biological reason for this could not be elucidated before more thorough experimental data were available.

\subsection{Expansion of the experimental database}

Much sought-after experimental data were obtained from more extensive investigations of the infection dynamics during the production of human influenza vaccine in adherent MDCK cells carried out by Schulze-Horsel et al. [2009]. An important additional feature was the flow cytometric determination of apoptotic cells by measuring the fragmentation of host cell DNA via TUNEL staining (see below).

The term apoptosis was introduced by Kerr et al. [1972] and denotes the most common form of programmed cell death [Alberts et al., 2008]. Apoptosis is a highly regulated process, which is prevalent in (and not confined to) animal cells and tissues [ibid.]. It is initiated by an abundance of external and internal stimuli and serves the purpose of relieving the organism of cells which are either no longer needed, irreversibly damaged, or potentially hazardous (e.g. virally infected) [ibid.]. In bioreactors apoptosis often leads to cell lysis and has major influence on the process productivity. On the intracellular level apoptosis is characterized by an amplifying proteolytic cascade which is mediated by special enzymes called caspases [ibid.]. As one of the last and decisive steps of the proteolytic cascade activated caspase 3 cleaves ICAD (inhibitor of caspase-activated deoxyribonuclease) and, thereby, liberates CAD (caspase-activated DNase), which migrates into the nucleus and starts to degrade chromatin by cutting DNA into nucleosome-sized pieces [Enari et al., 1998; Sakahira et al., 1998; Nagata, 2000]. Note, ICAD and CAD are also referred to as the $45 \mathrm{kDa}$ and $40 \mathrm{kDa}$ subunits of DFF (DNA fragmentation factor), respectively [Liu et al., 1997, 1998].

The fragmentation of DNA is considered one of the most characteristic features of apoptosis [Nagata, 2000]. The resulting double and single strand breaks of nuclear DNA can be measured via TdT-mediated dUTP nick end labeling (TUNEL), a staining method which was established by Gavrieli et al. [1992]. In this method the polymerization ability of TdT (terminal deoxyribonucleotidyl transferase) is used to catalyze the addition of dUTP (deoxyuridine triphosphate) nucleotides to $3^{\prime}$ DNA ends. Hence, every DNA 
strand break is labeled with a nucleotide chain of certain length leading to a specific chain length distribution which, in general, obeys the Poisson distribution [Bollum, 1978; Grosse and Manns, 1993]. To quantify the amount of added dUTP the latter is associated with a marker molecule. In the case of Schulze-Horsel et al. [2009] the fluorochrome TMR red (tetramethylrhodamine) was applied to allow for simultaneous measurements of the intracellular amount of viral protein with the help of FITC-labeled antibodies.

In contrast to Schulze-Horsel et al. [2008], this time not a mixture of antibodies was used to determine the amount of intracellular viral protein. Instead, exclusively monoclonal antibodies against influenza A virus nucleoprotein (NP) were applied [Schulze-Horsel et al., 2009]. Hence, the aforementioned virus equivalent (VE) accordingly consists of the average number of NP molecules which make up a virus particle (1000, Lamb and Krug [2001]). Another improvement in comparison to the publication of Schulze-Horsel et al. [2008] is the discrimination between infectious (active) and uninfectious (inactive) virus particles by adding tissue culture infectious dose $\left(\right.$ TCID $\left._{50}\right)$ titration [Hierholzer and Killington, 1996] as a quantification method for infectious virions or plaque forming units $(\mathrm{PFU})$. In addition, experimental data were collected at more frequent intervals facilitating a more informed interpretation.

Furthermore, the focus was strictly put on human influenza A virus strains. Namely, Schulze-Horsel et al. investigated the infection of adherent MDCK cell cultures with human influenza A/Puerto Rico/8/34 (H1N1) from two sources - from the Robert Koch Institute (RKI) in Berlin and from the National Institute for Biological Standards and Control (NIBSC) in Hertfortshire - and with a high growth reassortant of A/Wisconsin/67/2005 (H3N2, A/Puerto Rico/8/34 backbone). In the following these virus strains are denoted as A/H1N1-RKI, A/H1N1-NIBSC and A/H3N2, respectively.

\subsection{Influence of apoptosis}

In the light of the new experimental data the hypothesis formed that the influence of apoptosis is responsible for the occurrence of a second replication phase as proposed by the "two-phase model". Namely, it was assumed that the observed reversal of the propagation direction (the so called backshift) is the result of a general alteration of the viral replication mechanism caused by the induction of the apoptotic chain reaction at some point after the infection.

Influenza virus is known to induce apoptosis in infected cells, which is an integral part of the defensive strategy against viral infections [Takizawa et al., 1993; Hinshaw et al., 1994]. Hence, Kurokawa et al. [1999] suggested that for successful replication influenza virus has to rely on circumventing the antiviral influence of apoptosis by means of rapid 
multiplication. Since then, the role of apoptosis during influenza virus infection has been reconsidered resulting in a more complex picture of the interaction between virus and host. Hence, influenza A virus neither simply outruns apoptosis nor merely delays the onset or progress of the apoptotic chain reaction. In fact, the genome of influenza A virus codes for anti- as well as proapoptotic proteins that manipulate apoptotic and other cell signaling pathways in order to support efficient replication [Schultz-Cherry et al., 2001; Zhirnov et al., 2002; Lowy, 2003; Wurzer et al., 2003, 2004; Morris et al., 2005; Brydon et al., 2005; Ludwig et al., 2006; Ehrhardt et al., 2007; Halder et al., 2011; Tran et al., 2013]. However, progress within the apoptotic network is not stopped completely, so that eventually the point of no return is reached and the internucleosomal fragmentation of host cell DNA takes place leading to the destruction of the cell.

It is important to note that several hours can pass between the induction of apoptosis and the eventual fragmentation of DNA [Takizawa et al., 1993; Hinshaw et al., 1994; Earnshaw, 1995; Kurokawa et al., 1999; Zhirnov and Klenk, 2007]. Moreover, investigations of single cells exposed to tumor necrosis factor (TNF) or TNF-related apoptosis-inducing ligand (TRAIL) as apoptotic triggers revealed a pronounced cell-to-cell variability in the time elapsing between the induction of apoptosis and the final commitment to cell death [Albeck et al., 2008; Spencer et al., 2009]. In fact, the entire apoptotic process can be divided into two stages. The first stage starts with the induction of apoptosis and can last for several hours (in some special cases even days) [Green, 2005]. In contrast, the completion of the second stage, which is initialized by the so called mitochondrial outer membrane permeabilization (MOMP), takes only several minutes and is prominently concluded by DNA fragmentation [Green, 2005; Bialik et al., 2010]. With respect to the model of Müller et al. [2013] this motivated the assumption that infected cells stay capable of viral protein production and virus release in the first stage of apoptosis whose beginning supposedly coincides with the transition to the second phase of replication.

Furthermore, Zhirnov et al. [1999] reported that human influenza A nucleoprotein is cleaved by host cell caspases. However, the cleavage primarily takes place at the $\mathrm{N}$ terminus of NP molecules which are already part of viral ribonucleoprotein (RNP) complexes. As mentioned earlier, experimental results indicate that the employed antibodies are no longer able to bind to viral NP as soon it is associated with RNPs [Arranz et al., 2012; Moeller et al., 2012], so that the flow cytometric measurement of viral NP is not influenced by the caspase-dependent cleavage of viral nucleoprotein. Instead, an increased formation of RNPs seems to be responsible for the depletion of intracellular NP that characterizes the backshift during the second phase of replication.

Consequently, one could propose that the induction of apoptosis is associated with an increased formation of RNPs leading to the distinct backshift of the cell distribution. Unfortunately, the experimental setup employed by Schulze-Horsel et al. [2009] turns out to be not suitable to verify this hypothesis. This is because Schulze-Horsel et al. [2009] 
measured the fragmentation of host cell DNA by means of TUNEL staining. However, DNA fragmentation is a late event in apoptosis, which starts at a random point in time several hours after the initial induction of apoptosis by some kind of apoptotic trigger. Therefore, DNA fragmentation is initialized too late to be associated with the backshift of the cell distribution. Hence, the approval of the aforementioned hypothesis has to remain pending for the time being as additional experiments with markers for an early phase of apoptosis would be required.

\subsection{Process description and model assumptions}

In principle, the conditions and procedures are comparable to the ones described in Sections 2.1 and 3.2. Nevertheless, existing differences are presented in the following. For a detailed description of the applied experimental methods see Schulze-Horsel et al. [2009], and Schulze-Horsel [2011].

Adherent MDCK cells are grown in a small-scale bioreactor (1.21 working volume) on microcarriers where they eventually form confluent monolayers [Genzel et al., 2004]. After removal of spent medium and a washing step, fresh medium is added and cells are infected with the virus seed containing infectious/active and uninfectious/inactive virus particles. Active virions attach to uninfected cells and infect them subsequently. Although inactive virus particles can principally bind to the cell membrane and incorporation (albeit without successful replication) is possible, this is not considered in the model. Thus, inactive virus particles are assumed to simply accumulate in the supernatant without any interaction with the host cell population or further degradation. For simplicity, further differentiation between virus particles is omitted, i.e. defective interfering particles (DIPs) are neglected. As before, newly infected cells undergo an eclipse and latent phase which are assumed to coincide. After completion of the combined eclipse/latent phase infected cells start to produce virus protein and eventually release active as well as inactive virus particles into the medium. Any released active virus particles are assumed to exclusively attach to still uninfected cells or to degenerate to the inactive state. It is assumed that apoptotic cells predominantly arise from infected cells that have completed their latent phase. Furthermore, apoptotic cells are considered to be in an early stage of apoptosis, in which they are still capable of viral protein production and virus particle release until inevitable disintegration takes place.

The distribution dynamics of the process are determined by the temporal change of the intracellular amount of NP which is accessible to the fluorescein-conjugated antibodies against viral nucleoprotein. As previously noted, this seems to be exclusively applicable to newly produced NP which has not yet been bound in RNP complexes. Hence, with respect to the model viral protein production and RNP complexation are responsible 


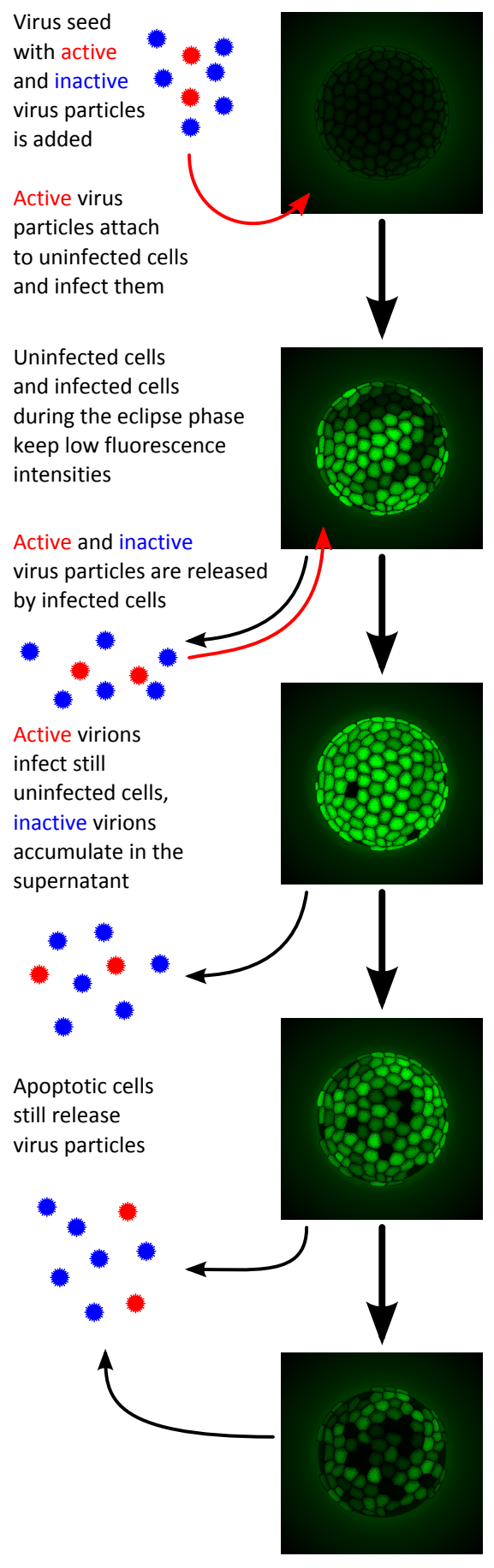

Uninfected cells on microcarrier surface

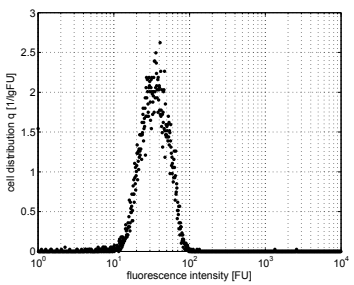

Infected cells start to produce viral protein (e.g. NP) after the eclipse phase

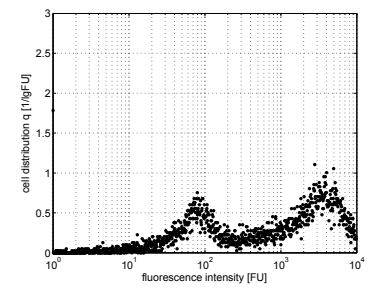

The fluorescence intensity of infected cells increases; infected cells start to become apoptotic and eventually detach from the surface

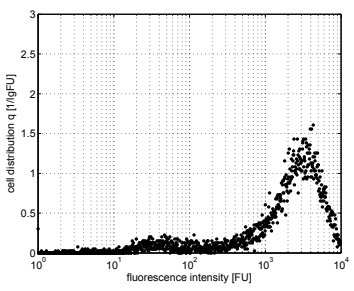

The fluorescence intensity of apoptotic cells decreases; more cells detach

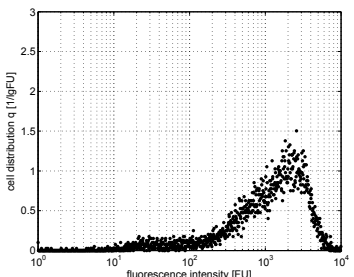

More apoptotic cells detach from the microcarrier and eventually undergo cell lysis

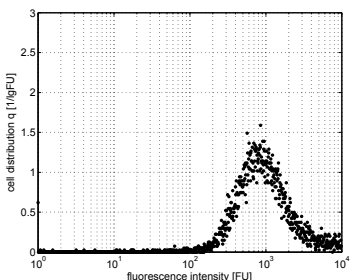

Figure 24: Illustration of the virus replication process. The main phases of the process appear in chronological order from top to bottom. The left column of illustrations shows stylized fluorescence microscopic pictures of cells growing on the surface of a microcarrier. The right column contains cell distributions from an experiment with A/H3N2 [Schulze-Horsel, 2011] which are representative for the described phases. 
for the distribution dynamics as measured by flow cytometry. In order to reproduce the distributed experimental data it is required to specifically consider two phases of viral replication. In the first phase viral protein production exceeds the formation of RNP complexes, so that the intracellular amount of free viral NP increases. In the second replication phase the conditions are reversed with the formation of RNP complexes exceeding viral protein production. In consequence, free intracellular NP is depleted leading to the characteristic backshift of the cell distribution at later times. It is assumed that the transition from one phase of replication to the other is triggered by the induction of apoptosis.

Note, that growth, death, and apoptosis of uninfected cells are considered to have only minor influence on the process and are therefore neglected. Moreover, it is assumed that cells do not disintegrate before the induction of apoptosis.

In Fig. 24 the main phases of the process are depicted in comparison to flow cytometric results taken from an experiment with A/Wisconsin/67/2005 (H3N2).

\subsection{Model equations}

As an initial condition, all cells are considered to be uninfected and log-normally distributed with respect to the degree of fluorescence. As before, the overall concentration $U_{c, 0}$, the expectation $\mu_{0}$ and the standard deviation $\sigma_{0}$ are applied (see Eqs. (3.8) and (3.11) on pages 21 and 23 , respectively).

On contact with active virus particles uninfected cells become infected, which is regulated by the infection coefficient $k_{\mathrm{vi}}$. At time of infection contact-inhibited MDCK cells form confluent monolayers and cell division is only possible after detachment of infected cells. Furthermore, the specific cell death rate due to cultivation conditions is much lower than the specific cell death rate due to infection [Möhler et al., 2005]. Therefore, both influences are being neglected. Hence the dynamics of uninfected cells can be described as

$$
\frac{\partial \tilde{U}_{c}(t, \varphi)}{\partial t}=-k_{\mathrm{vi}} \tilde{U}_{c}(t, \varphi) V_{\mathrm{ac}}(t) .
$$

Directly after infection the cells undergo the latent phase of length $\tau$, during which no virus replication and no virus release take place. This lag period lumps together events happening before the synthesis of NP, e.g. the entry of the virus into the host cell, uncoating of the nucleic acid, various transport processes and RNA transcription.

$$
\frac{\partial \tilde{L}_{c}(t, \varphi)}{\partial t}=k_{\mathrm{vi}} \tilde{U}_{c}(t, \varphi) V_{\mathrm{ac}}(t)-k_{\mathrm{vi}} \tilde{U}_{c}(t-\tau, \varphi) V_{\mathrm{ac}}(t-\tau)
$$




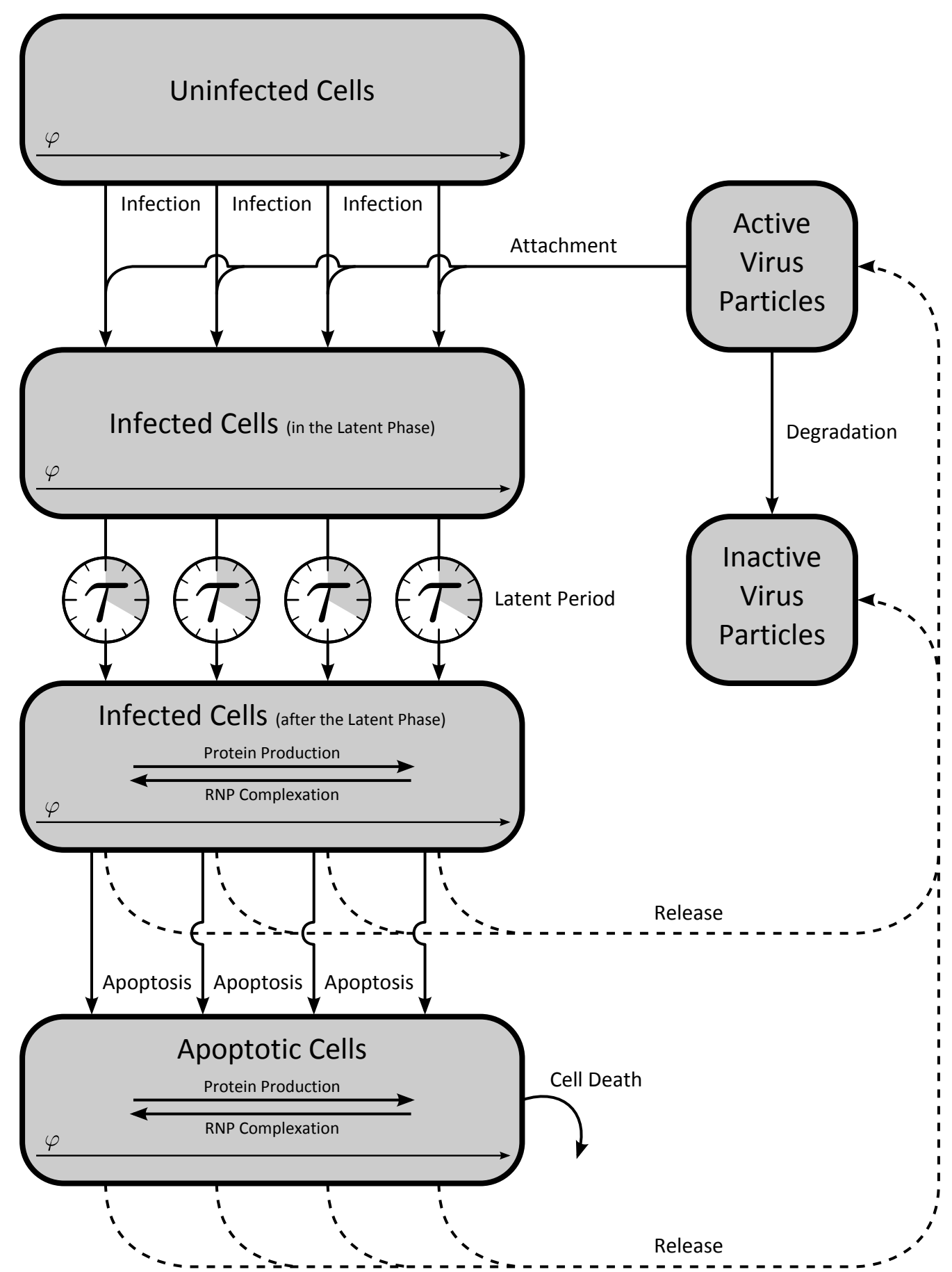

Figure 25: Scheme of the model of Müller et al. [2013]. All cells are distributed over the degree of fluorescence $\varphi$. While uninfected cells keep their degree of fluorescence, infected cells once they have passed the latent phase - and apoptotic cells can move along the internal coordinate $\varphi$ by means of viral protein production and RNP complex formation. Both, infected (after the latent phase) and apoptotic cells release virus particles, of which only active virions attach to uninfected cells. 
Note, that Eq. (5.2) corresponds to Eq. (3.12) on page 23 for $k_{\mathrm{cdv}}=0$, i.e. cell death during the latent phase is not taken into account in the present model.

After completion of the lag period infected cells start to synthesize viral NP with the protein production coefficient $k_{\text {pro }}(\varphi)$, which causes the degree of fluorescence to rise. On the other hand, $\varphi$ is reduced by binding of NP during the formation of RNP complexes with the NP binding coefficient $k_{\text {bind }}(\varphi)$. Both effects are combined into the net coefficient $k_{\text {net }}(\varphi)=k_{\text {pro }}(\varphi)-k_{\text {bind }}(\varphi)$. As for infected cells viral protein production is faster than RNP complexation, $k_{\text {net }}$ is always positive so that the degree of fluorescence of an infected cell generally increases. After infection cells tend to become apoptotic with the apoptosis coefficient $k_{\text {apo }}(\varphi)$.

$$
\frac{\partial \tilde{I}_{c}(t, \varphi)}{\partial t}=k_{\mathrm{vi}} \tilde{U}_{c}(t-\tau, \varphi) V_{\mathrm{ac}}(t-\tau)-\frac{\partial}{\partial \varphi}\left(k_{\mathrm{net}}(\varphi) \tilde{I}_{c}(t, \varphi)\right)-k_{\mathrm{apo}}(\varphi) \tilde{I}_{c}(t, \varphi)
$$

The majority of apoptotic cells originate from infected cells [Schulze-Horsel et al., 2009], i.e. apoptosis of uninfected cells is neglected. As discussed above, apoptotic cells are considered to be in an early state of apoptosis, in which they are still capable of virus replication and release. However, it is assumed that due to a yet unspecified influence of apoptosis the formation of RNP complexes is intensified, so that NP binding exceeds the production. Thus, the net coefficient of apoptotic cells $k_{\text {net,apo }}(\varphi)=k_{\text {pro,apo }}(\varphi)-$ $k_{\text {bind,apo }}(\varphi)$ turns out to be negative, which leads to a generally decreasing degree of fluorescence. Cell death is accounted for by the respective coefficient $k_{\mathrm{cd}}(\varphi)$.

$$
\frac{\partial \tilde{A}_{c}(t, \varphi)}{\partial t}=k_{\text {apo }}(\varphi) \tilde{I}_{c}(t, \varphi)-\frac{\partial}{\partial \varphi}\left(k_{\text {net }, \mathrm{apo}}(\varphi) \tilde{A}_{c}(t, \varphi)\right)-k_{\mathrm{cd}}(\varphi) \tilde{A}_{c}(t, \varphi)
$$

Active as well as inactive virus particles are released by infected cells after they have passed through the latent phase and apoptotic cells alike. The ratio between active and inactive virions is regulated by the plating efficiency $P_{\text {eff }} \in[0,1]$. Only active virions are supposed to attach to uninfected cells and successfully infect them. It is assumed that every active virus particle is infective, so that the attachment coefficient corresponds to the infection coefficient. Furthermore, active virions degrade to inactive ones with the degradation coefficient $k_{\mathrm{deg}}$.

$$
\frac{\mathrm{d} V_{\mathrm{ac}}(t)}{\mathrm{d} t}=P_{\text {eff }} k_{\mathrm{rel}} \int_{\varphi}\left(\tilde{I}_{c}(t, \varphi)+\tilde{A}_{c}(t, \varphi)\right) \mathrm{d} \varphi-k_{\mathrm{vi}} V_{\mathrm{ac}}(t) \int_{\varphi} \tilde{U}_{c}(t, \varphi) \mathrm{d} \varphi-k_{\mathrm{deg}} V_{\mathrm{ac}}(t)
$$

Inactive virus particles are either released by infected and apoptotic cells or originate from active virions. As any interaction with host cells and further degradation are neglected, inactive virus particles simply accumulate in the supernatant.

$$
\frac{\mathrm{d} V_{\text {inac }}(t)}{\mathrm{d} t}=\left(1-P_{\text {eff }}\right) k_{\text {rel }} \int_{\varphi}\left(\tilde{I}_{c}(t, \varphi)+\tilde{A}_{c}(t, \varphi)\right) \mathrm{d} \varphi+k_{\mathrm{deg}} V_{\mathrm{ac}}(t)
$$


The resulting model structure is presented in Figure 25.

\subsection{Numerical solution}

As already proposed for the extended model of Müller et al. [2008] (see Section 3.4, page $25 \mathrm{f}$.), uninfected cells and infected cells during the latent phase are assumed to be unable to change their degree of fluorescence. Hence, Eqs. (3.15) and (3.16) hold and Eq. (5.1) (page 56) can be simplified to

$$
\frac{\mathrm{d} U_{\mathrm{c}}(t)}{\mathrm{d} t}=-k_{\mathrm{vi}} U_{\mathrm{c}}(t) V_{\mathrm{ac}}(t)
$$

As before the latent period is represented by a transport system of constant length, which infected cells have to pass through before viral replication is initialized (see Section 3.4 and Appendix B (page 99)). Cells enter the transport system upon infection, travel along the transport coordinate $\lambda \in \mathbb{R},[0,1]$, and leave after the duration $\tau$ has elapsed

$$
\begin{aligned}
& \frac{\partial \hat{L}_{\mathrm{c}}(t, \lambda)}{\partial t}=-\frac{1}{\tau} \frac{\partial \hat{L}_{\mathrm{c}}(t, \lambda)}{\partial \lambda} \\
& \text { with } \quad \hat{L}_{\mathrm{c}}(t, \lambda=0)=\tau \cdot k_{\mathrm{vi}} U_{\mathrm{c}}(t) V_{\mathrm{ac}}(t) .
\end{aligned}
$$

Note, that Eq. 5.8 corresponds to Eq. (3.18) on page 25 for $k_{\mathrm{cdv}}=0$.

The discretization of Eq. 5.8 via finite-volume method (FVM) is similar to the one described in Appendix C.2 (page 102 ff.) for $k_{\mathrm{cdv}}=0$ :

$$
\begin{gathered}
\frac{\mathrm{d} \hat{L}_{\mathrm{c}, i}(t)}{\mathrm{d} t}=-\frac{1}{\Delta \lambda \tau}\left(\hat{L}_{\mathrm{c}, i}(t)-\hat{L}_{\mathrm{c}, i-1}(t)\right) \\
\text { with } \quad \hat{L}_{\mathrm{c}, 0}(t)=\tau \cdot k_{\mathrm{vi}} U_{\mathrm{c}}(t) V_{\mathrm{ac}}(t) .
\end{gathered}
$$

This time the number of control volumes is set to $M=1024$ to approximate a hard delay.

After the transport system is traversed cells of species $\hat{L}_{\mathrm{c}}$ change over to species $\tilde{I}_{\mathrm{c}}$. When looking at the last control volume of Eq. (5.9) $(i=M)$ the change of the cell concentration due to cells leaving the latent phase at time $t$ equals $\hat{L}_{\mathrm{c}, M}(t) / \tau$. By multiplication with the normalized initial distribution $q_{\mathrm{sim}, 0}(\varphi)$ this flux constitutes the source of the infected cells after the latent phase. Therefore, the respective balance equation is changed to:

$$
\frac{\partial \tilde{I}_{c}(t, \varphi)}{\partial t}=\frac{\hat{L}_{\mathrm{c}, M}(t)}{\tau} q_{\mathrm{sim}, 0}(\varphi)-\frac{\partial}{\partial \varphi}\left(k_{\mathrm{net}}(\varphi) \tilde{I}_{c}(t, \varphi)\right)-k_{\text {apo }}(\varphi) \tilde{I}_{c}(t, \varphi)
$$




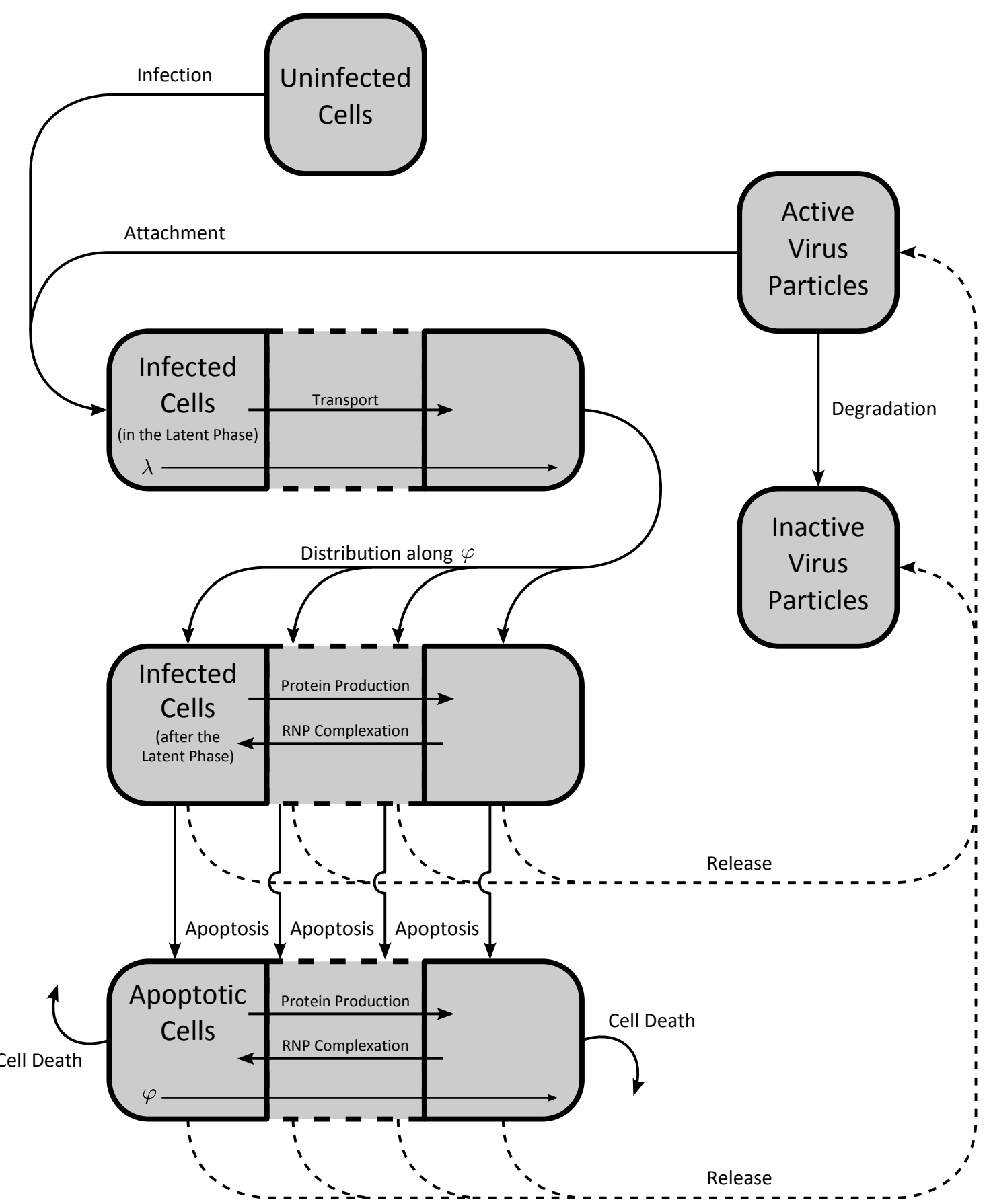

Figure 26: Scheme of the discretized model of Müller et al. [2013]. Uninfected and infected cells in the latent phase are assumed to not change their degree of fluorescence $\varphi$. Therefore, they can be implemented as lumped species with regard to $\varphi$. The latent phase is approximated by a discretized transport system with the transport coordinate $\lambda$. When cells reach the end of the transport system they change over to the species of productive infected cells. By applying the normalized initial cell distribution, infected cells which have passed the latent phase are distributed along $\varphi$. 
Eq. (5.10) is discretized on a logarithmic grid with $N=128$ control volumes (see Appendix C.4, page 106):

$$
\begin{aligned}
& \frac{\mathrm{d} \tilde{I}_{\mathrm{c}, i}(t)}{\mathrm{d} t}=\frac{\hat{L}_{\mathrm{c}, M}(t)}{\tau} q_{\mathrm{sim}, 0, i}-\frac{1}{\Delta \varphi_{i}}\left(k_{\mathrm{net}, i} \tilde{I}_{\mathrm{c}, i}(t)-k_{\mathrm{net}, i-1} \tilde{I}_{\mathrm{c}, i-1}(t)\right)-k_{\mathrm{apo}, i} \tilde{I}_{\mathrm{c}, i}(t) \\
& \text { with } \quad \tilde{I}_{\mathrm{c}, 0}(t)=\tilde{I}_{\mathrm{c}, N}(t)=0 .
\end{aligned}
$$

Likewise, the balance equation of the apoptotic cells, Eq. (5.4), is discretized (see Appendix C.5, page 107) yielding

$$
\begin{aligned}
& \frac{\mathrm{d} \tilde{A}_{\mathrm{c}, i}(t)}{\mathrm{d} t}=k_{\mathrm{apo}, i} \tilde{I}_{\mathrm{c}, i}(t)-\frac{1}{\Delta \varphi_{i}}\left(k_{\mathrm{net}, \mathrm{apo}, i+1} \tilde{A}_{\mathrm{c}, i+1}(t)-k_{\mathrm{net}, \mathrm{apo}, i} \tilde{A}_{\mathrm{c}, i}(t)\right)-k_{\mathrm{cd}, i} \tilde{A}_{\mathrm{c}, i}(t) \\
& \text { with } \tilde{A}_{\mathrm{c}, 1}(t)=\tilde{A}_{\mathrm{c}, N+1}(t)=0 .
\end{aligned}
$$

The virus balance equations are reformulated accordingly:

$$
\begin{aligned}
& \frac{\mathrm{d} V_{\mathrm{ac}}(t)}{\mathrm{d} t}=P_{\mathrm{eff}} k_{\mathrm{rel}} \sum_{i=1}^{N}\left(\tilde{I}_{\mathrm{c}, i}(t)+\tilde{A}_{\mathrm{c}, i}(t)\right) \Delta \varphi_{i}-k_{\mathrm{vi}} U_{\mathrm{c}}(t) V_{\mathrm{ac}}(t)-k_{\mathrm{deg}} V_{\mathrm{ac}}(t), \\
& \frac{\mathrm{d} V_{\mathrm{inac}}(t)}{\mathrm{d} t}=\left(1-P_{\mathrm{eff}}\right) k_{\mathrm{rel}} \sum_{i=1}^{N}\left(\tilde{I}_{\mathrm{c}, i}(t)+\tilde{A}_{\mathrm{c}, i}(t)\right) \Delta \varphi_{i}+k_{\mathrm{deg}} V_{\mathrm{ac}}(t) .
\end{aligned}
$$

The resulting model structure is presented in Fig. 26.

\subsection{Parameter estimation}

Parameter estimation was performed by Robert Dürr as part of the joint publication of Müller et al. [2013]. Here, a summary of the procedure is given by which the kinetic parameters of the model were determined from experimental data collected by SchulzeHorsel et al. [2009]. To translate the emerging infinite dimensional parameter estimation problem to a finite dimension the parameters are assumed to depend linearly on the internal coordinate $\varphi$. For a nonlinear dependency on the degree of fluorescence see Dürr et al. [2012]. 


\section{Transformation of flow cytometric data}

As the model is solved numerically on a logarithmic grid with $N=128$ finite control volumes (see Section 5.5), the measurements are transformed to the same grid by simply averaging the cell numbers over eight sensor channels at a time. In addition, a transformation is made to include the total cell concentrations (uninfected, infected and apoptotic cells) obtained from independently performed cell counts

$$
c_{i}\left(t_{k}\right)=\frac{\sum_{j=8 i-7}^{8 i} Z_{j}\left(t_{k}\right)}{\sum_{j} Z_{j}\left(t_{k}\right)} C_{\text {tot }}\left(t_{k}\right) \quad \text { for } i \in \mathbb{N},[1,128] \text { and } j \in \mathbb{N},[1,1024] .
$$

Here, $c_{i}\left(t_{k}\right)$ is the cell concentration in control volume $i, Z_{j}\left(t_{k}\right)$ is the number of cells assigned to sensor channel $j$ and $C_{\text {tot }}\left(t_{k}\right)$ is the total cell concentration in the reactor obtained from cell counts at sample time $t_{k}$.

\section{Translation of the inverse problem to a finite dimension}

In order to adapt the presented model to experimental data an inverse problem has to be solved. This problem is set in an infinite dimension as the parameters characterizing viral protein replication, apoptosis and cell lysis depend on the internal coordinate $\varphi$. Two general solution approaches are known from literature to translate this kind of problem into a finite dimension. In the first one, the functional dependency is assumed to be known and can be described by an analytical function with a few scalar parameters, e.g. a gaussian distribution characterized by mean and variance [Sherer et al., 2008]. If the shape of the function is not known a priori, it can be parametrized with a suitable approximation, e.g. piecewise constant, piecewise linear or spline approximation, to obtain a finite dimensional representation [Luzyanina et al., 2009; Banks et al., 2011]. In the present contribution the corresponding kinetic parameters are assumed to depend linearly on the degree of fluorescence.

$$
k_{*}(\varphi)=\frac{k_{*}\left(\varphi_{\max }\right)-k_{*}\left(\varphi_{\min }\right)}{\varphi_{\max }-\varphi_{\min }}\left(\varphi-\varphi_{\min }\right)+k_{*}\left(\varphi_{\min }\right)
$$

\section{Parameter estimation setup}

The vector of unknown parameters is given by

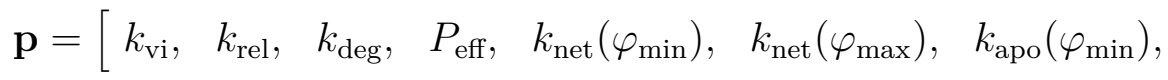

$$
\begin{aligned}
& \left.k_{\text {apo }}\left(\varphi_{\max }\right), \quad k_{\text {net,apo }}\left(\varphi_{\min }\right), \quad k_{\text {net,apo }}\left(\varphi_{\max }\right), \quad k_{\text {cd }}\left(\varphi_{\min }\right), \quad k_{\text {cd }}\left(\varphi_{\max }\right)\right] .
\end{aligned}
$$


In addition to flow cytometric distribution data, the concentrations of active and inactive virions are considered yielding the measurement vector

$$
y\left(t_{k}\right)=\left(\begin{array}{c}
c_{1}\left(t_{k}\right) \\
\vdots \\
c_{128}\left(t_{k}\right) \\
V_{\mathrm{ac}}\left(t_{k}\right) \\
V_{\text {inac }}\left(t_{k}\right)
\end{array}\right)
$$

The parameters are now estimated in a weighted least squares framework

$$
\min _{\mathbf{p}} \sum_{k=1}^{n_{t}}\left[y\left(t_{k}\right)-y_{\mathrm{ref}}\left(t_{k}, \mathbf{p}\right)\right] \mathbf{w}\left[y\left(t_{k}\right)-y_{\mathrm{ref}}\left(t_{k}, \mathbf{p}\right)\right],
$$

in which the weighting vector is chosen according to the measurement variance [Englezos and Kalogerakis, 2001]

$$
\begin{aligned}
& \mathbf{w}=\operatorname{diag}\left(\sigma_{i}\left(t_{k}\right)^{-2}\right), \\
& \text { with } \quad \sigma_{1 \ldots 128}\left(t_{k}\right)=\max _{\forall k, i=1 \ldots 128}\left(y_{i}\left(t_{k}\right)\right), \\
& \text { and } \quad\left(\begin{array}{l}
\sigma_{129}\left(t_{k}\right) \\
\sigma_{130}\left(t_{k}\right)
\end{array}\right)=\left(\begin{array}{l}
\max _{\forall k}\left(y_{129}\left(t_{k}\right)\right) \\
\max _{\forall k}\left(y_{130}\left(t_{k}\right)\right)
\end{array}\right) .
\end{aligned}
$$

This nonlinear least squares problem is solved with the MATLAB [2012] optimization routine fmincon, which uses a sequential quadratic programming algorithm. As this method can trap in local minima for non-convex objective functions, several runs with different initial guesses were performed.

Due to the complexity of the parameter estimation procedure, particularly caused by the high sensitivity of the cost function on the delay, a two-step procedure was pursued. At first, the length of the latent phase $\tau$ was fixed and all other parameters were estimated. The initial values were taken from Müller et al. [2011]. In a second step, all parameters with the exception of $\tau$ were set to the estimated values of the first step and $\tau$ was estimated.

In Table IV the parameter sets for one experiment with A/H1N1-RKI and two independent experiments with A/H3N2 are summarized. All sets differ quantitatively, but show qualitative similarity with respect to the trends of the linearly dependent parameters. Initial conditions were derived from experimental data [Schulze-Horsel et al., 2009; Schulze-Horsel, 2011]. 


\begin{tabular}{|c|c|c|c|c|}
\hline \multirow[t]{2}{*}{ Parameter } & \multicolumn{3}{|c|}{ Experiment } & \multirow[t]{2}{*}{ Unit } \\
\hline & A/H1N1-RKI & A/H3N2, Exp. 1 & A/H3N2, Exp. 2 & \\
\hline$F_{\mathrm{VE}}$ & 2.6 & 2.645 & 2.464 & $\mathrm{FU}$ \\
\hline$k_{\mathrm{apo}}\left(\varphi_{\min }\right)$ & $0.238 \cdot 10^{-1}$ & $9.654 \cdot 10^{-1}$ & $8.36 \cdot 10^{-1}$ & $\mathrm{~h}^{-1}$ \\
\hline$k_{\text {apo }}\left(\varphi_{\max }\right)$ & 1.178 & 19.21 & 6.237 & $h^{-1}$ \\
\hline$k_{\mathrm{cd}}\left(\varphi_{\min }\right)$ & $4.132 \cdot 10^{-2}$ & $2.863 \cdot 10^{-2}$ & $3.239 \cdot 10^{-2}$ & $\mathrm{~h}^{-1}$ \\
\hline$k_{\mathrm{cd}}\left(\varphi_{\max }\right)$ & & 0 & & $\mathrm{~h}^{-1}$ \\
\hline$k_{\mathrm{deg}}$ & 0.045 & 0.143 & $9.63 \cdot 10^{-2}$ & $\mathrm{~h}^{-1}$ \\
\hline$k_{\text {net }}\left(\varphi_{\min }\right)$ & $0.132 \cdot 10^{3}$ & $3.207 \cdot 10^{3}$ & $3.536 \cdot 10^{3}$ & $\mathrm{~h}^{-1}$ \\
\hline$k_{\text {net }}\left(\varphi_{\max }\right)$ & $1.232 \cdot 10^{3}$ & $1.313 \cdot 10^{4}$ & $4.683 \cdot 10^{3}$ & $\mathrm{~h}^{-1}$ \\
\hline$k_{\text {net,apo }}\left(\varphi_{\min }\right)$ & -0.65 & -0.87 & -1.1 & $\mathrm{~h}^{-1}$ \\
\hline$k_{\text {net,apo }}\left(\varphi_{\max }\right)$ & -122.6 & -48.36 & -70.4 & $\mathrm{~h}^{-1}$ \\
\hline$k_{\text {rel }}$ & 324.3 & 597.2 & 783.8 & $h^{-1}$ \\
\hline$k_{\mathrm{vi}}$ & $1.16 \cdot 10^{-6}$ & $6.337 \cdot 10^{-7}$ & $9.583 \cdot 10^{-7}$ & $\mathrm{ml} / \mathrm{h}$ \\
\hline$P_{\text {eff }}$ & $5.177 \cdot 10^{-2}$ & $3.568 \cdot 10^{-2}$ & $2.33 \cdot 10^{-2}$ & - \\
\hline$\tau$ & 4 & 5 & 4.75 & $\mathrm{~h}$ \\
\hline$I_{\mathrm{c}, 0}$ & & 0 & & $\mathrm{ml}^{-1}$ \\
\hline$U_{\mathrm{c}, 0}$ & $1.77 \cdot 10^{6}$ & $1.65 \cdot 10^{6}$ & $1.09 \cdot 10^{6}$ & $\mathrm{ml}^{-1}$ \\
\hline$V_{\mathrm{ac}, 0}$ & $7.6 \cdot 10^{3}$ & $7.6 \cdot 10^{3}$ & $3.2 \cdot 10^{3}$ & $\mathrm{ml}^{-1}$ \\
\hline$V_{\text {inac }, 0}$ & $3.724 \cdot 10^{5}$ & $3.724 \cdot 10^{5}$ & $1.568 \cdot 10^{5}$ & $\mathrm{ml}^{-1}$ \\
\hline$\mu_{0}$ & 35.2 & 34.8 & 45.1 & $\mathrm{FU}$ \\
\hline$\sigma_{0}$ & 0.165 & 0.188 & 0.175 & $\mathrm{FU}$ \\
\hline
\end{tabular}

Table IV: Parameter sets and initial conditions for the model of Müller et al. [2013]. Parameters were fitted to experimental data from three independent experiments with A/H1N1-RKI and A/H3N2, respectively [Schulze-Horsel et al., 2009; Schulze-Horsel, 2011].

\subsection{Simulation results}

In this section simulation results are compared with experimental data collected by Schulze-Horsel et al. [2009] for three independent experiments with A/H1N1-RKI and A/H3N2, respectively. In Table IV the applied parameter set and initial conditions are summarized. Note, that Schulze-Horsel et al. [2009] also used a variant of A/Puerto Rico/8/34 from NIBSC in Hertfortshire (A/H1N1-NIBSC) whose experimental results differ significantly from the other two virus strains. Therefore, the case of A/H1N1NIBSC is discussed separately in Section 5.8 (page 78 ff.). 


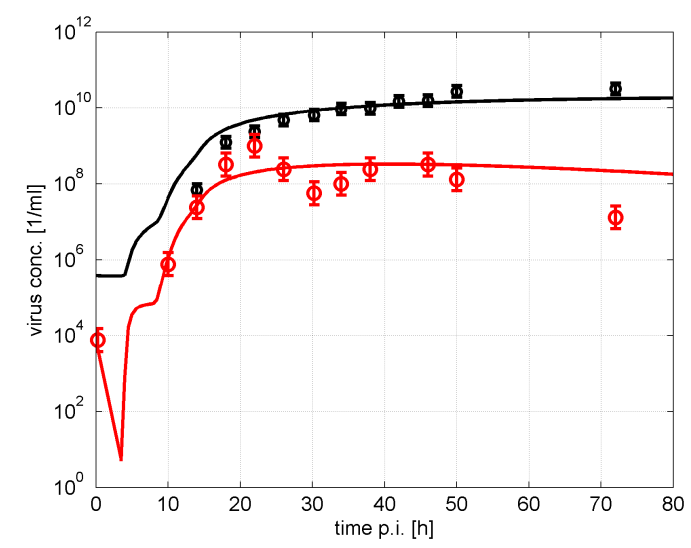

a) A/H1N1-RKI

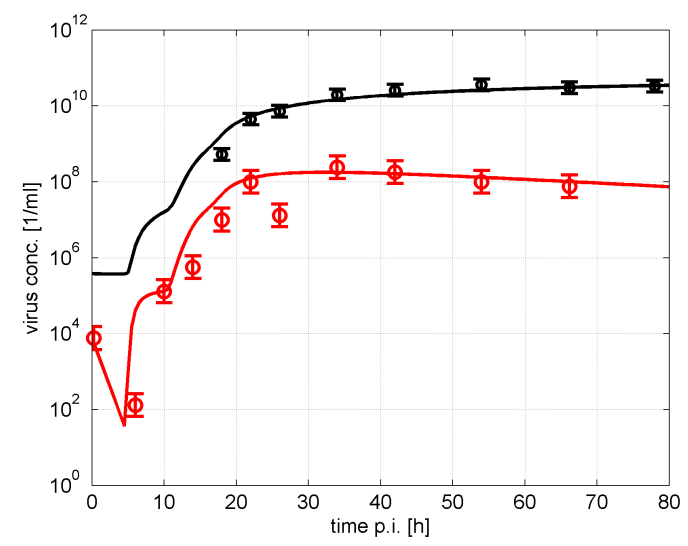

b) A/H3N2, Experiment 1

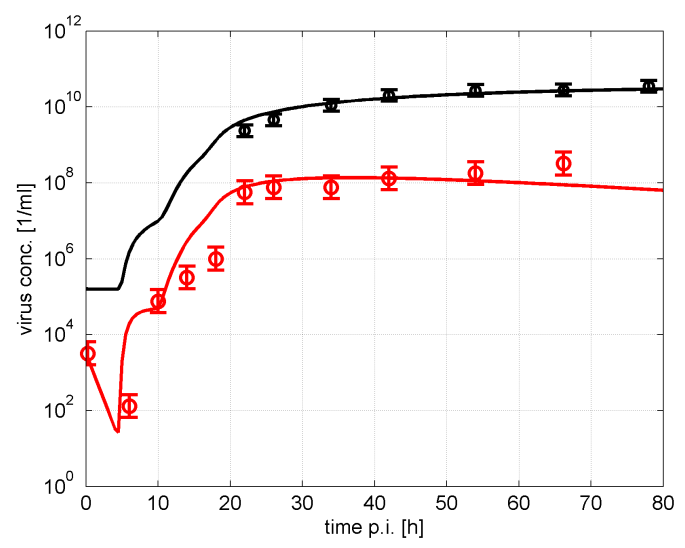

c) A/H3N2, Experiment 2

Figure 27: Comparison of the integral dynamics of the model of Müller et al. [2013] with experimental data of Schulze-Horsel et al. [2009] for the infection of adherent MDCK cells with A/H1N1RKI and A/H3N2 - lines represent simulation results for the total virus concentration (black) and the active virus concentration (red); circles represent experimental virus titers of HA measurements (black) and TCID $_{50}$ titration (red) including standard error bars of the respective method $\left( \pm 0.15 \frac{\mathrm{lg} \mathrm{HA}}{100 \mathrm{\mu l}}\right.$ and $\left.\pm 0.3 \mathrm{lg} \mathrm{PFU} / \mathrm{ml}\right)$. The applied parameter set and initial conditions are summarized in Tab. IV (page 64).

\section{Integral dynamics}

In Fig. 27 simulation results of the temporal change of the total virus concentration and the active virus concentration are compared with experimental results. For the comparison the HA measurements have to be converted with Eq. (3.25) on page 29. According to Schulze-Horsel et al. [2009] the limits of detection of the HA assay and the TCID $_{50}$ titration amount to $2 \cdot 10^{7}$ virusparticles $/ \mathrm{ml}$ and $3.2 \cdot 10^{2} \mathrm{PFU} / \mathrm{ml}$, respectively. Because of the logarithmic scale of the ordinate, experimental results that equal zero are 
not depicted. Thus, the first three to five measurements of the total virus titer do not show up in Fig. 27.

Note, that due to an improved workflow the standard error of the HA assay has been decreased from $\pm 0.3 \frac{\mathrm{lg} \mathrm{HA}}{100 \mathrm{\mu l}}$ to $\pm 0.15 \frac{\mathrm{lg} \mathrm{HA}}{100 \mathrm{\mu l}}$ [Schulze-Horsel et al., 2009] setting higher requirements for a good fit between simulation and experiment. Hence, in the first $20 \mathrm{~h}$ p.i. the fit turns out to be less accurate with respect to the total virus concentrations. This stems from the assumption that eclipse phase and latent phase coincide, which produces a trade-off between the fit of the integral dynamics and the distribution dynamics resulting in a too small $\tau$ with respect to the integral dynamics. Although, for A/H1N1-RKI the length of the latent period seems to be quite correct as far as the incline of the active virus concentration is concerned.

After the virus seed is added active virus particles attach to uninfected cells and are taken up by means of endocytosis. As for all three experiments the infection coefficients $k_{\mathrm{vi}}$ are rather low in comparison to the ones used for the experiments with equine influenza (see Section 3.5, page 28), the distinct drop of the active virus concentration happens to have a rather moderate slope, which nicely corresponds to the experimental data - the second measurement of the experiment with A/H1N1-RKI equals zero and is therefore not visible. The total virus concentration starts below the detection limit of the HA assay and stays constant for the length of the latent period of the first generation of infected cells. As soon as the latter begin to release virus particles, both virus titers increase in two distinct steps which start at around 5 and $10 \mathrm{~h}$ p.i., respectively. These steps mark the relatively abrupt increase of the productive host cell population each time a new generation of infected cells completes the latent phase. Though, because of the necessarily limited number of measurements, the experimental determination of the exact time of emergence of a new generation of productive cells is not possible.

While the total virus concentration keeps on increasing, which is true for the simulation and the HA assay and underlines the assumption of a negligible degradation of inactive virus particles, the course of the $\mathrm{TCID}_{50}$-values seems to be less decided between the different experiments. For A/H1N1-RKI and the first experiment of A/H3N2 the active virus concentrations reach their maximum at about $22 \mathrm{~h} \mathrm{p.i.} \mathrm{and} \mathrm{generally} \mathrm{decrease} \mathrm{from}$

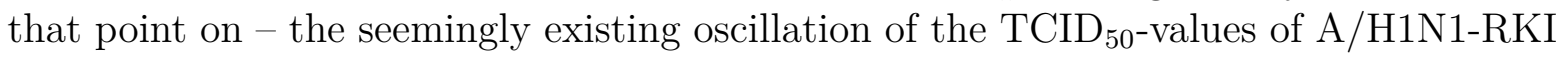
for the time period between 20 and $50 \mathrm{~h}$ p.i. stems most likely from assay and sampling limitations. With respect to the simulation the decline of the active virus concentration sets in as the degradation of active virus particles gains more influence, due to an ever decreasing number of productive cells. While this seems to be in good agreement with the first experiment of $\mathrm{A} / \mathrm{H} 3 \mathrm{~N} 2$, the predicted decline turns out to be insufficient for A/H1N1-RKI. On the other hand, the TCID T0 measurements of the second experiment with A/H3N2 suggest that the active virus concentration can continue to rise for a prolonged period of time. However, this could easily be resolved by considering a variable 
plating efficiency $P_{\text {eff }}$, which defines the ratio of active virus particles, as it is very likely that the ability to produce effective virions is subject to change during the progress of infection, e.g. by limitations in the supply of precursors of viral components, deterioration of cells, and the influence of defective interfering particles (DIPs). However, with respect to the integral dynamics of the first experiment with $\mathrm{A} / \mathrm{H} 3 \mathrm{~N} 2$ a constant plating efficiency seems to be appropriate.

In general, the simulation results show reasonable agreement with the experimental data. Though, there are some difficulties in predicting the exact appearance time of the initial rise. This presumably stems from the assumption of a combined eclipse and latent phase, which constitutes a kind of enforced conformity between the integral and the distribution dynamics. Another option would be to consider the release rate $k_{\text {rel }}$ as being dependent on the inner state of an infected cell. For instance, one could think of a virus release rate which starts out low in order to reach a maximum value during the progress of the process and possibly declines again at a later stage.

\section{Distribution dynamics}

In Figure 28 the mean fluorescence intensities (MFI) of the simulation are compared with experimental MFIs for A/H1N1-RKI and A/H3N2. For all three experiments a sharp increase of the MFI can be detected at about $10 \mathrm{~h}$ p.i. In the simulations this increase is preceded by either a small step $(\mathrm{A} / \mathrm{H} 3 \mathrm{~N} 2)$ or a moderate incline $(\mathrm{A} / \mathrm{H} 1 \mathrm{~N} 1)$, which both start around $5 \mathrm{~h}$ p.i. due to the appearance of the first productive cells. Approximately at $20 \mathrm{~h}$ p.i. the maximum MFI is reached and a more or less pronounced decline sets in. Although the MFI is a rather crude measure for the course of the distribution dynamics, it provides a general overview and delivers a first indication for the quality of the fit. The backshift of the cell distributions is clearly visible and, with exception of the last measurement of A/H1N1-RKI, also quite nicely reproduced.

In Figures 29 to 35 (page $71 \mathrm{ff}$.) simulated cell distributions are compared with their experimental counterparts for A/H1N1-RKI and A/H3N2. While the experimental cell distributions are determined by Eq. (3.5) (page 19), the simulated cell distributions are calculated from the scaled and normalized sum of the individual cell distributions of all 


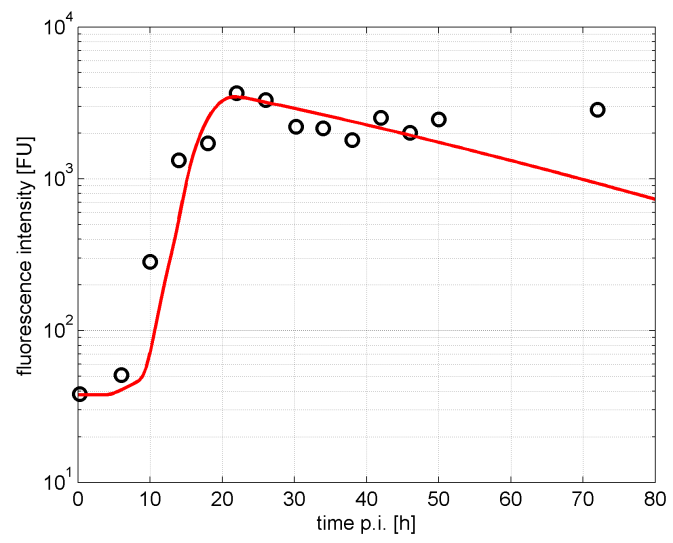

a) A/H1N1-RKI

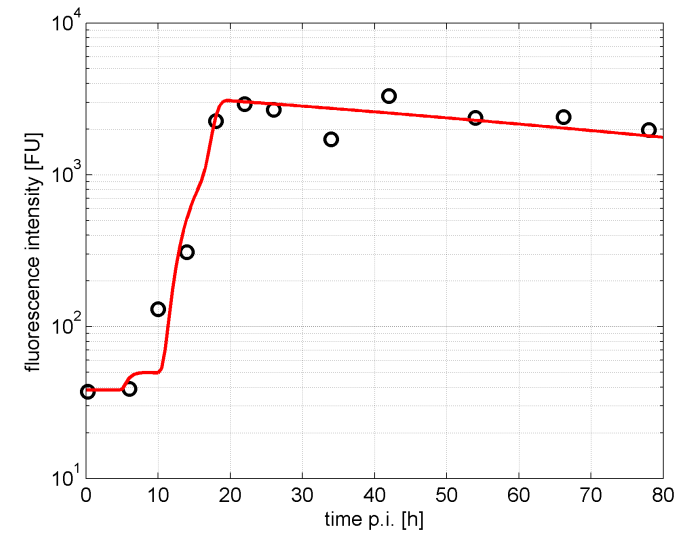

b) A/H3N2, Experiment 1

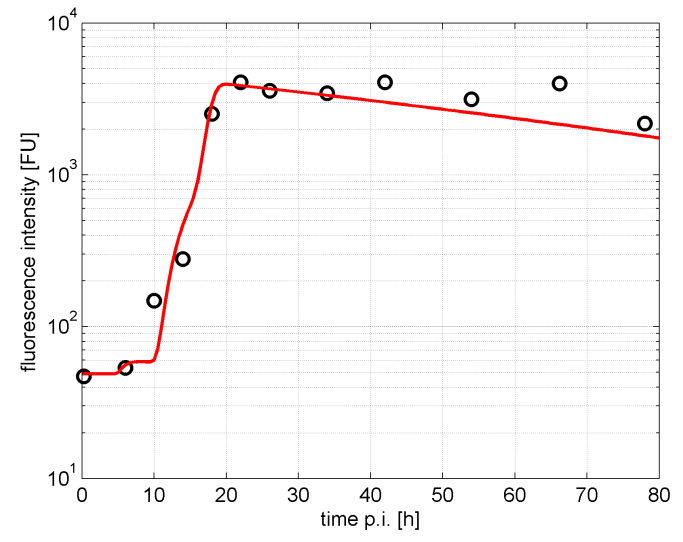

c) A/H3N2, Experiment 2

Figure 28: Comparison of the mean fluorescence intensities (MFIs) of the model of Müller et al. [2013] with experimental data of Schulze-Horsel et al. [2009] for three independent experiments temporal changes of the simulated MFIs are represented by lines; experimental MFIs are represented by circles. 
cell populations:

$$
\begin{aligned}
& q_{\mathrm{c}, i}(t)=\frac{\left(U_{\mathrm{c}}(t)+\Delta \lambda \sum_{j=1}^{M} L_{\mathrm{c}, j}(t)\right) q_{\mathrm{sim}, i, 0}+I_{\mathrm{c}, i}(t)+A_{\mathrm{c}, i}(t)}{U_{\mathrm{c}}(t)+\Delta \lambda \sum_{j=1}^{M} L_{\mathrm{c}, j}(t)+\sum_{j=1}^{N} \Delta \varphi_{j}\left(I_{\mathrm{c}, j}(t)+A_{\mathrm{c}, j}(t)\right)} \cdot \frac{\Delta \varphi_{i}}{\Delta F_{\mathrm{lg}}} \quad\left[\frac{1}{" \lg \mathrm{FU} "}\right] \\
& \text { for } \quad i \in \mathbb{N},[1, N] \quad \text { with } \quad \Delta F_{\mathrm{lg}}=\frac{4}{N} \quad[\text { "lg FU"]. }
\end{aligned}
$$

The distribution dynamics of A/H1N1-RKI are presented in Figs. 29 to 31. The experimental cell distribution at $0.25 \mathrm{~h}$ p.i. is considered to correspond to the initial cell distribution, because no change of the distribution is expected at such a short time post infection. As long as no cells have left the latent phase in order to initialize the production of NP and thereby increase the degree of fluorescence, the position of the initial distribution is fixed as far as the model is concerned. However, at 6 and $10 \mathrm{hp}$.i. the position of the experimental distribution shifts to slightly lower fluorescence intensities - this can also be observed for both experiments with A/H3N2 (Figs. 32 and 34). The cause of this effect is not clear.

The comparison at $10 \mathrm{~h}$ p.i. reveals that in the simulation the accumulation of viral NP proceeds too slow, i.e. $k_{\text {net }}$ is too small. The experimental results suggest that at that time p.i. the second generation of productive cells already should have reached regions of more than $10^{3} \mathrm{FU}$. At $14 \mathrm{~h}$ p.i. this impression is strengthened. In the model the second generation of productive cells has covered some distance, albeit not enough. It is followed by the third generation, which constitutes a middle peak, resulting in a trimodal distribution. Due to a larger cell-to-cell variability, e.g. with respect to the length of the latent period, or the rate of viral protein accumulation, the sequence of individual generations of productive cells is not as strictly outlined in the actual cell population, resulting in a more homogeneous distribution. Hence, the experiment is characterized by a bimodal cell distribution, with a left peak of not yet infected cells or cells in the latent phase and a right peak with productive cells which show an increased fluorescence intensity. The transient bimodality of the experimental cell distributions is a distinct feature of all human influenza A virus strains that were examined by SchulzeHorsel et al. [2009].

In the simulation virtually all cells have been infected and already left the latent phase at $18 \mathrm{~h}$ p.i. Though, in the experiments bimodality remains intact for at least another four hours. Basically, a small amount of cells seems to stick to the region of relatively low fluorescence intensity until $42 \mathrm{~h}$ p.i. These cells are either still uninfected, which is rather unlikely at later times, or they are infected but not or hardly productive. Reasons 
for this could either lie in a vast cell-to-cell variability or in the induction of an antiviral state by means of interferon (IFN) signaling. The latter assumption is also supported by experimental results for A/H1N1-NIBSC (see Section 5.8, page 78). As Seitz et al. [2010] show, the level of IFN expression strongly depends on the specific influenza virus strain. They also demonstrate that, though there is no significant effect on the final virus yield of the specific virus strain, manipulation of the IFN expression in MDCK cells has an influence on the intermediate dynamics of influenza virus replication.

As apoptosis is induced in more and more cells, the depletion of viral NP commences and the reversal of the propagation direction of the cell distribution takes place. At first the simulation rushes ahead somewhat ( $22 \mathrm{~h}$ p.i.), but afterwards, with exception of the last measurement $(72 \mathrm{~h}$ p.i.), it is in reasonable agreement with the experiment. The results at $72 \mathrm{~h}$ p.i. suggest that presumably due to a high variability being present in the cell population the backshift is not uniformly executed, resulting in a wide spread of cells over the fluorescence intensity axis, which cannot be reproduced by the model.

The distribution dynamics of the first experiment with A/H3N2 are presented in Figs. 32 and 33. In this case the second generation of productive cells proceeds fast enough along the degree of fluorescence $(14 \mathrm{~h}$ p.i.) - the cells of the first generation are too few to be noticed. Though, variability is higher in the experimental results leading to a more homogeneous spread over the fluorescence intensity. At $18 \mathrm{~h}$ p.i. a bimodal distribution can be seen for both simulation and experiment. However, the left peak of the experimental distribution changed its position to higher fluorescence intensities between 14 and $18 \mathrm{~h}$ p.i. This seems to suggest that the generation of infected cells that moved to fluorescence intensities larger than $10^{3} \mathrm{FU}$ between 14 and $18 \mathrm{~h}$ p.i. must have started their movement predominantly from the left flank of the main peak that existed at $14 \mathrm{~h}$ p.i. Another explanation could be that for later generations of infected cells viral NP production starts out in a more "cautious" manner due to the antiviral effect of IFN signaling, so that these cells accumulate NP very slowly at first in order to accelerate the viral protein production at a later stage. However, after $18 \mathrm{~h}$ p.i. only small residues are left at low fluorescence intensities. Note that the experimental cell distribution at $66.17 \mathrm{~h}$ p.i. bears some resemblance to the last measurement of $\mathrm{A} / \mathrm{H} 1 \mathrm{~N} 1-\mathrm{RKI}$ and to the last measurement of the alternative experiment with A/H3N2 (Fig. 35).

In principal, the second experiment with A/H3N2 is similar to the first one (Figs. 34 and 35). A transient bimodality is produced between 14 and $18 \mathrm{~h}$ p.i. and, with respect to the simulation, is resolved between 18 and $22 \mathrm{~h}$ p.i. As before, in the experiments a small amount of cells is maintained at low fluorescence intensities. After $22 \mathrm{~h}$ p.i. the backshift is performed. While the cell distribution stays coherent in the simulation, fluctuations caused by nonuniform behavior are detectable in the experimental results. However, considering the simple structure of the presented model, that lumps a complex system into only one internal coordinate, it should be no surprise that some issues remain. Especially 
at later times p.i. the agreement can be improved by the definition of parameters with a nonlinear dependency on the degree of fluorescence as it has been shown by Dürr et al. [2012]. Though, the potential of this approach is limited due to restrictions imposed by other modeling assumptions. In particular, interpretation of the respective parameter functions has to be treated with caution as the actual model structure has to be taken into account. Nonetheless, despite its simple structure the model is able to reproduce the characteristic dynamic phenomena - i.e. transient multimodality, and reversal of propagation direction - in reasonable agreement with experimental data of two of the considered virus strains.

The third virus strain used by Schulze-Horsel et al. [2009] is the NIBSC variant of A/Puerto Rico/8/34 (A/H1N1-NIBSC) which, for instance, can be distinguished from the RKI variant (A/H1N1-RKI) on the basis of the amino acid sequence of the viral nonstructural protein 1 (NS1) [Seitz et al., 2010]. Consequently, the distribution dynamics of A/H1N1-NIBSC differ from the ones previously published and shall therefore be discussed separately in the next section.

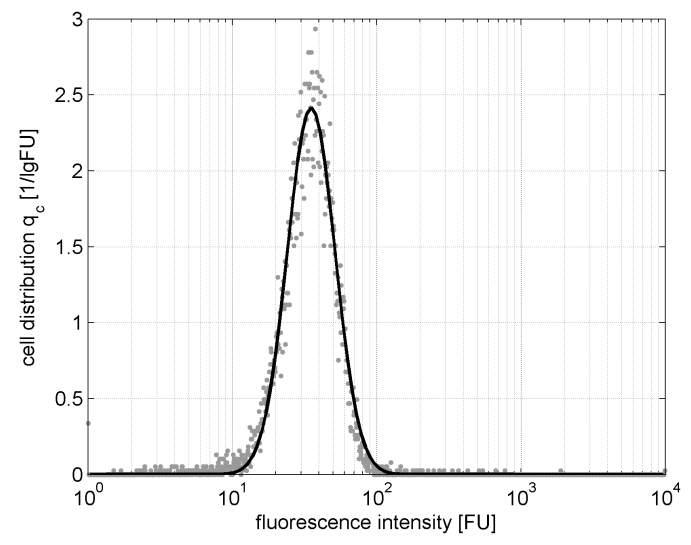

a) $t=0.25 \mathrm{~h}$ p.i.

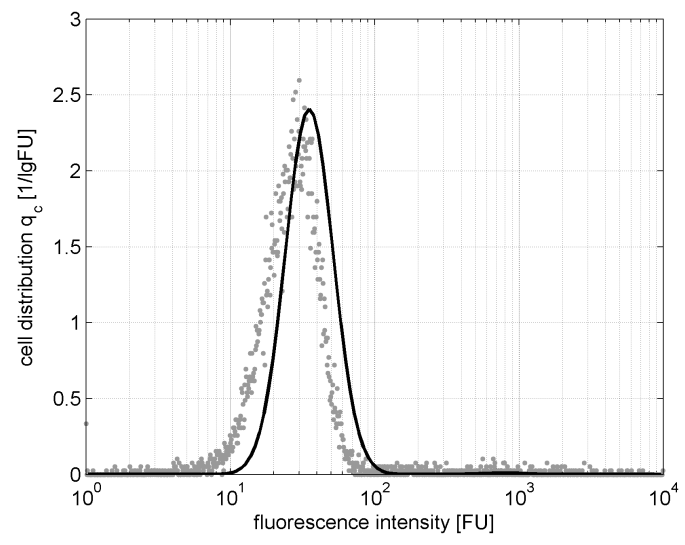

b) $t=6 \mathrm{~h}$ p.i.

Figure 29: Comparison of the distribution dynamics of the model of Müller et al. [2013] with experimental data of Schulze-Horsel et al. [2009] for A/H1N1-RKI (Part I) - simulated cell distributions are represented by lines; experimental cell distributions are represented by dots. 


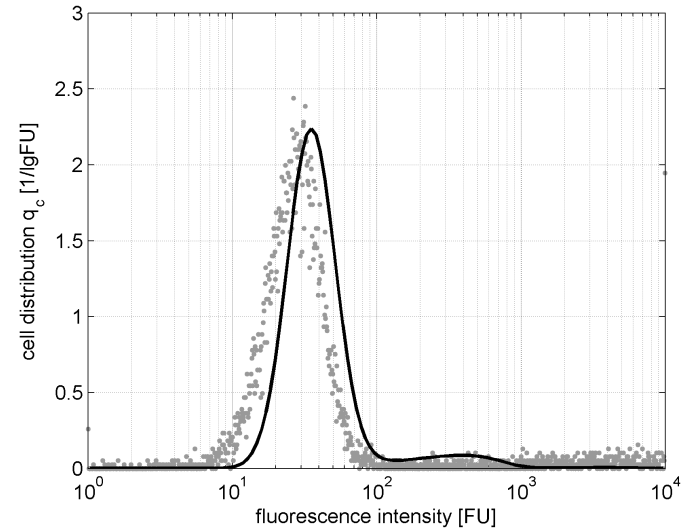

a) $t=10 \mathrm{~h} \mathrm{p.i.}$

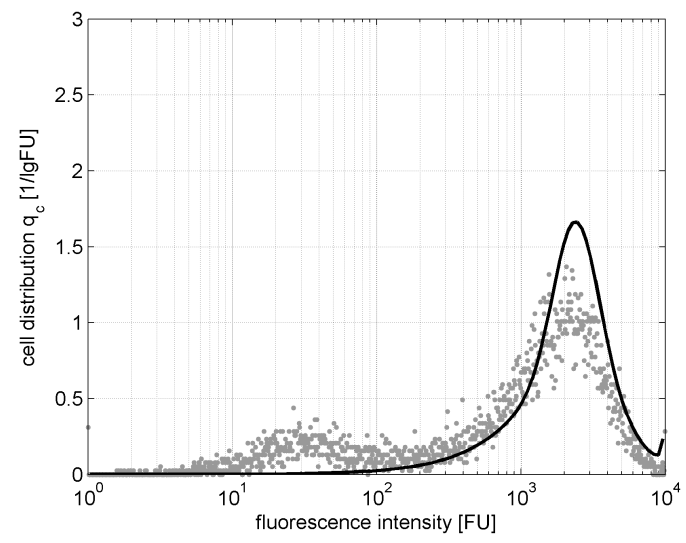

c) $t=18 \mathrm{~h}$ p.i.

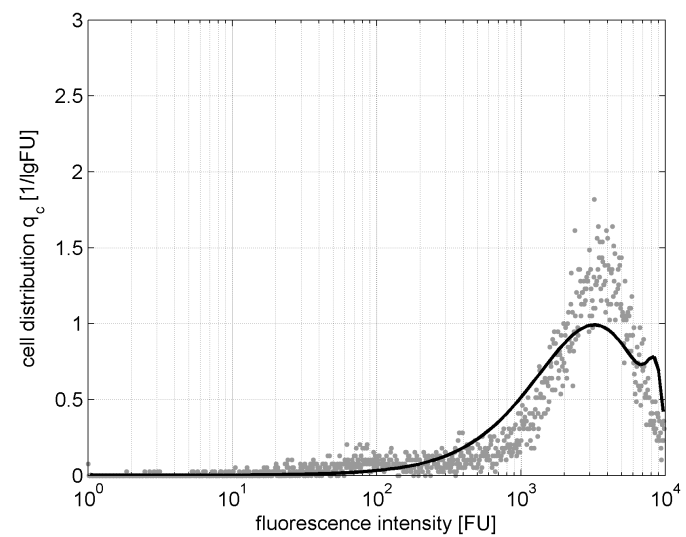

e) $t=26 \mathrm{~h}$ p.i.

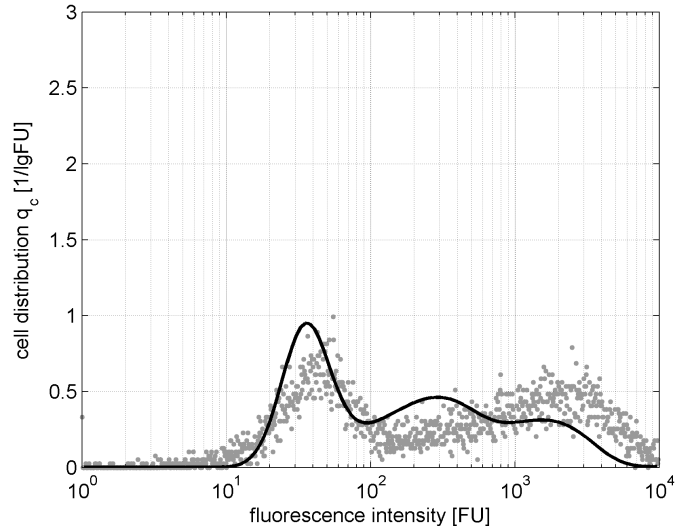

b) $t=14 \mathrm{~h}$ p.i.

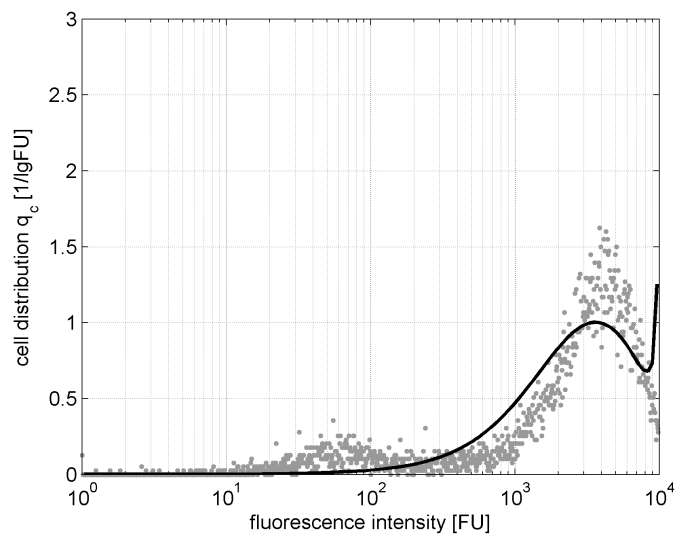

d) $t=22 \mathrm{~h}$ p.i.

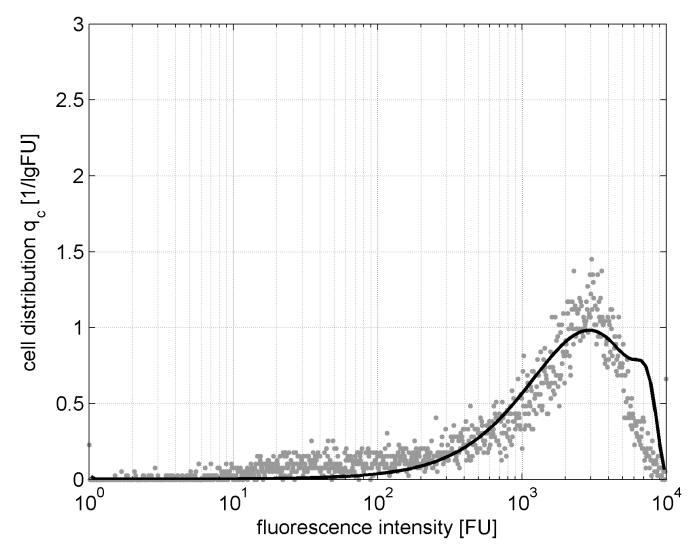

f) $t=30.25 \mathrm{~h}$ p.i.

Figure 30: Comparison of the distribution dynamics of the model of Müller et al. [2013] with experimental data of Schulze-Horsel et al. [2009] for A/H1N1-RKI (Part II) - simulated cell distributions are represented by lines; experimental cell distributions are represented by dots. 


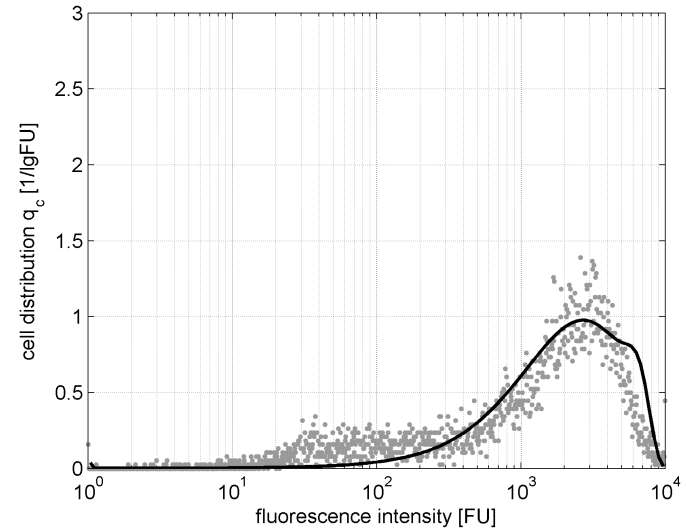

a) $t=34 \mathrm{~h} \mathrm{p.i.}$

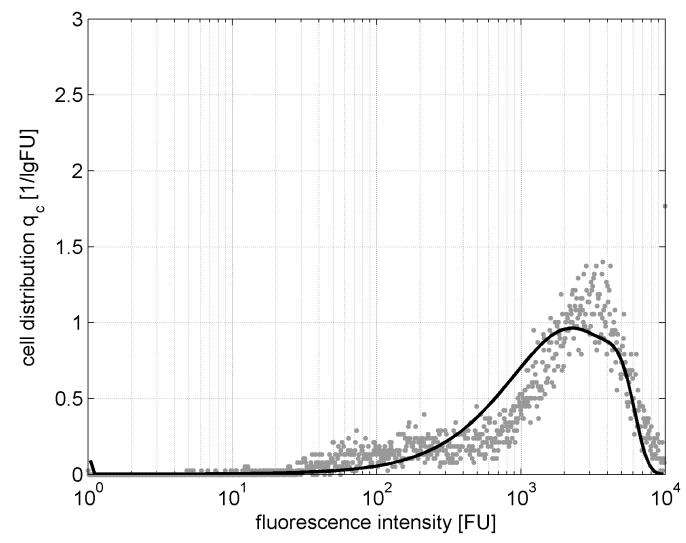

c) $t=42 \mathrm{~h}$ p.i.

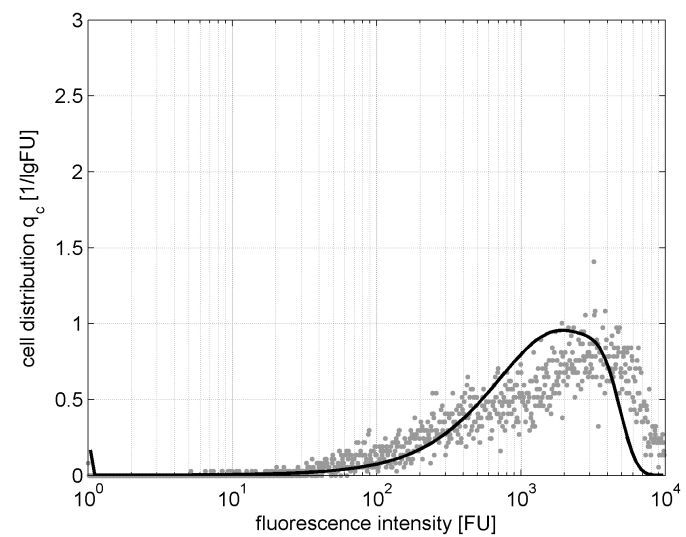

e) $t=50 \mathrm{~h}$ p.i.

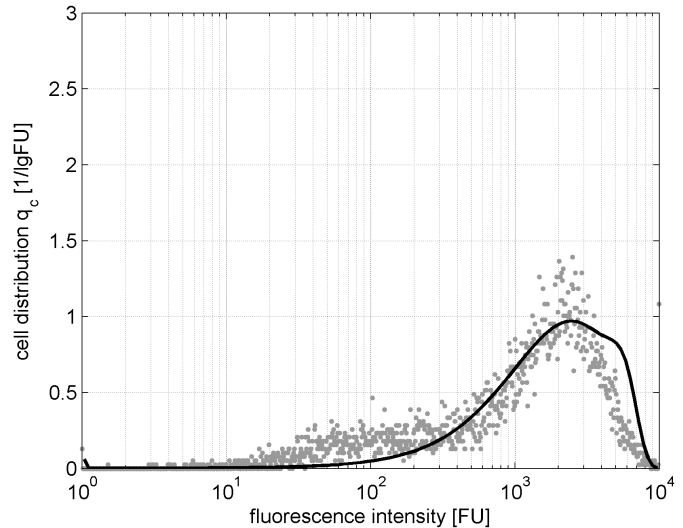

b) $t=38 \mathrm{~h}$ p.i.

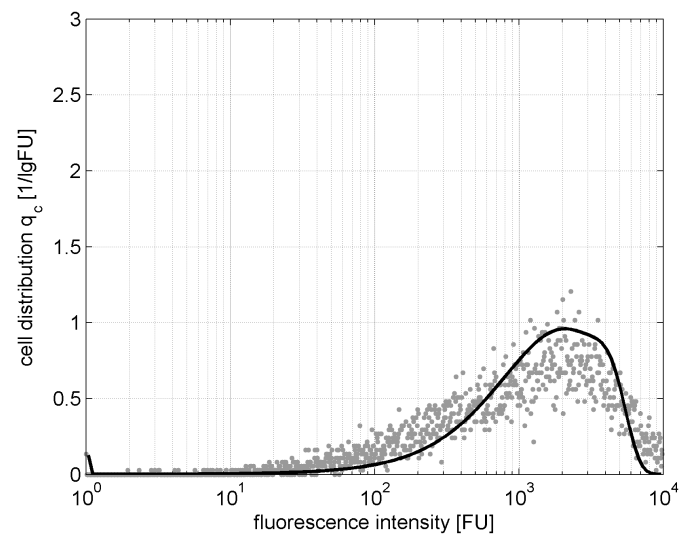

d) $t=46 \mathrm{~h}$ p.i.

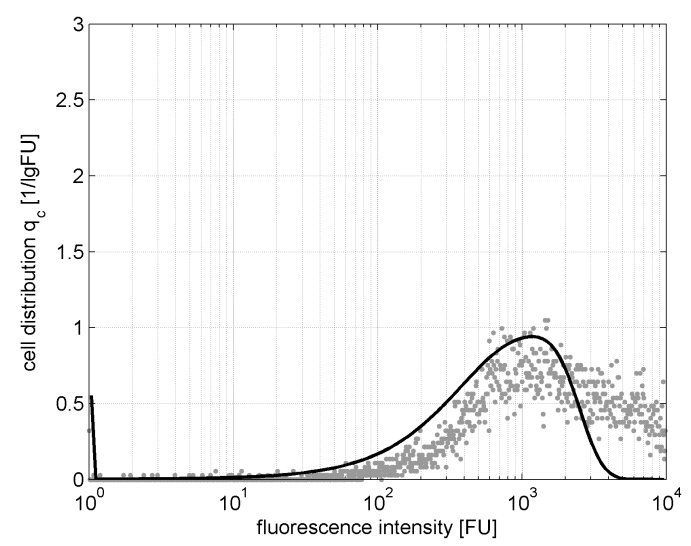

f) $t=72 \mathrm{~h}$ p.i.

Figure 31: Comparison of the distribution dynamics of the model of Müller et al. [2013] with experimental data of Schulze-Horsel et al. [2009] for A/H1N1-RKI (Part III) - simulated cell distributions are represented by lines; experimental cell distributions are represented by dots. 


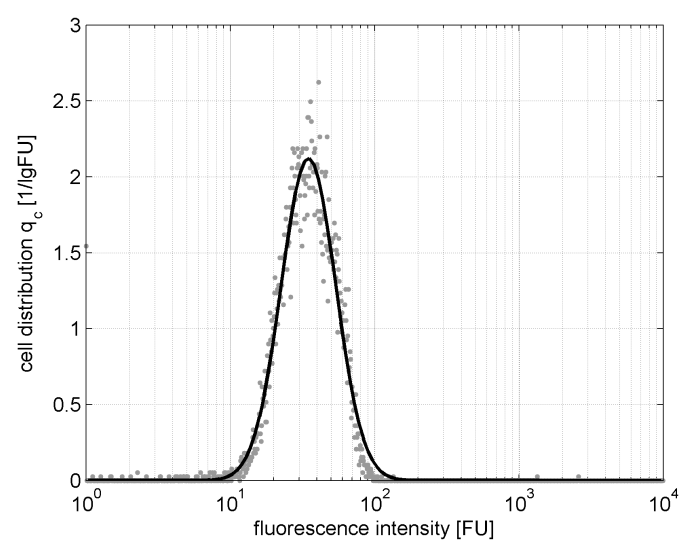

a) $t=0.25 \mathrm{~h}$ p.i.

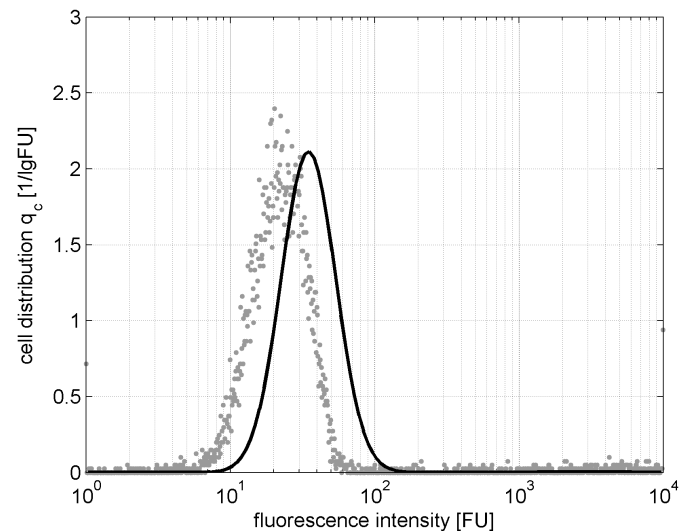

c) $t=10 \mathrm{~h}$ p.i.

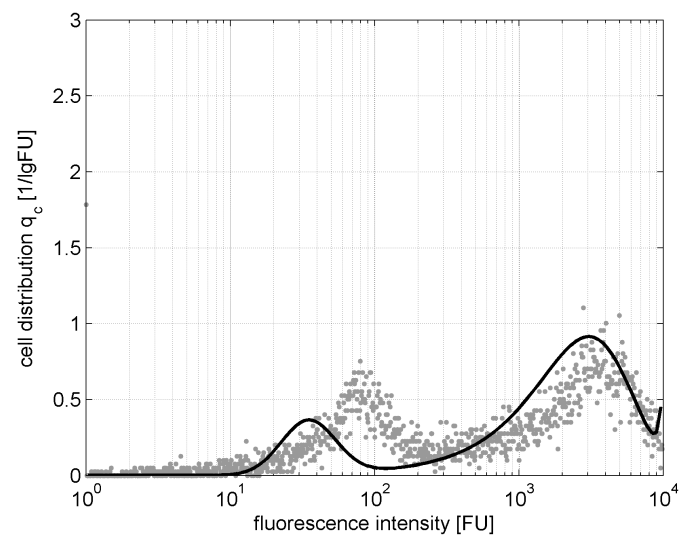

e) $t=18 \mathrm{hp}$.i.

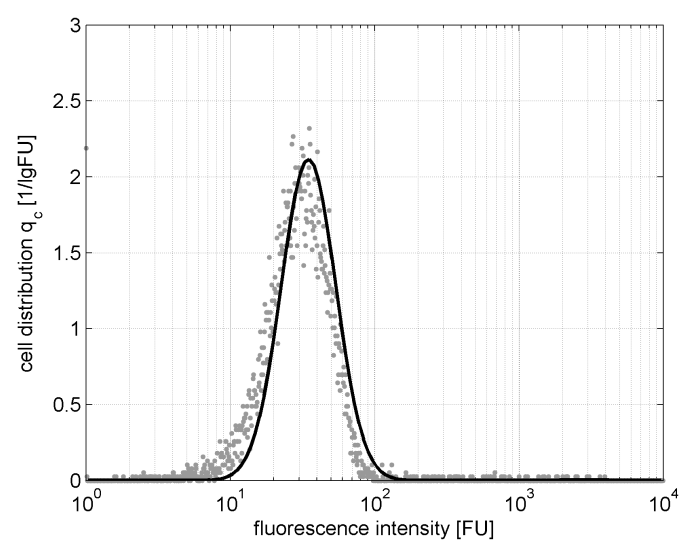

b) $t=6 \mathrm{~h}$ p.i.

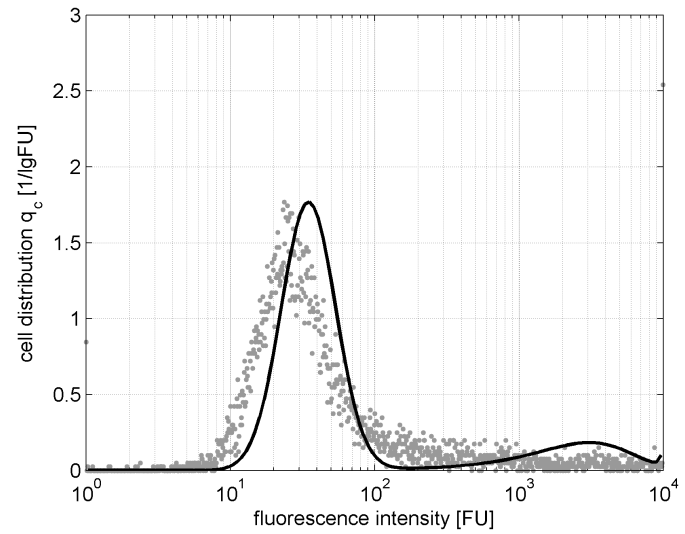

d) $t=14 \mathrm{~h}$ p.i.

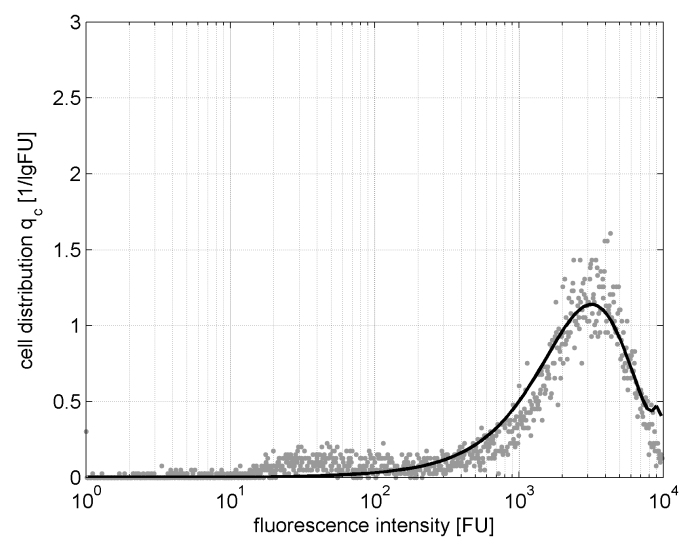

f) $t=22 \mathrm{~h} \mathrm{p.i.}$

Figure 32: Comparison of the distribution dynamics of the model of Müller et al. [2013] with experimental data of Schulze-Horsel et al. [2009] for A/H3N2, Experiment 1 (Part I) - simulated cell distributions are represented by lines; experimental cell distributions are represented by dots. 


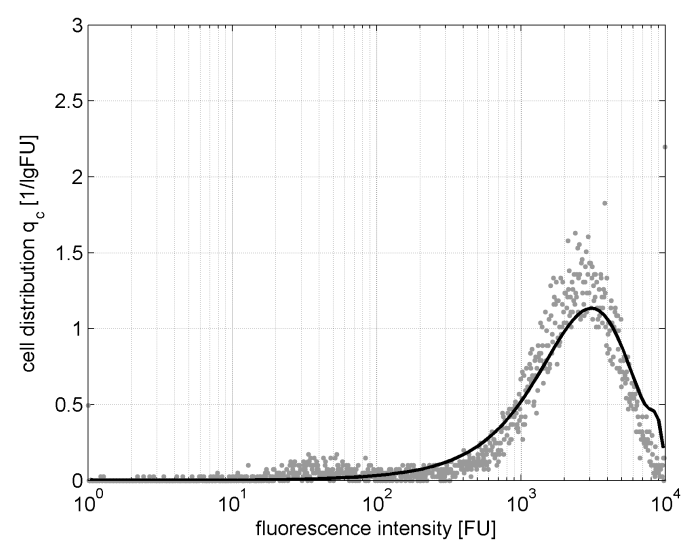

a) $t=26 \mathrm{~h}$ p.i.

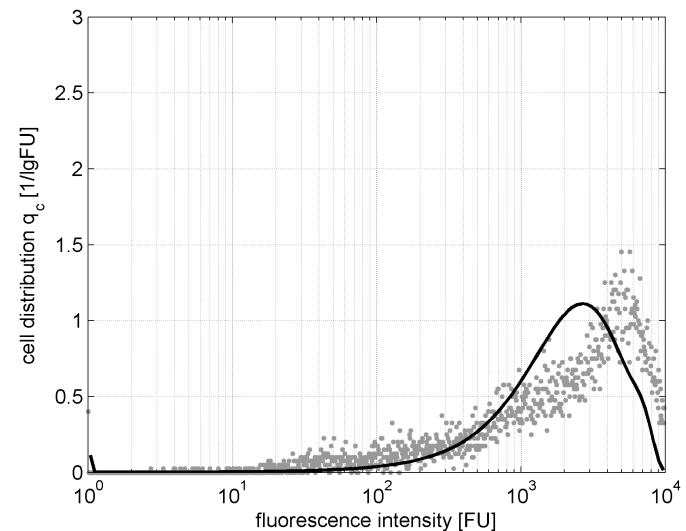

c) $t=42 \mathrm{~h}$ p.i.

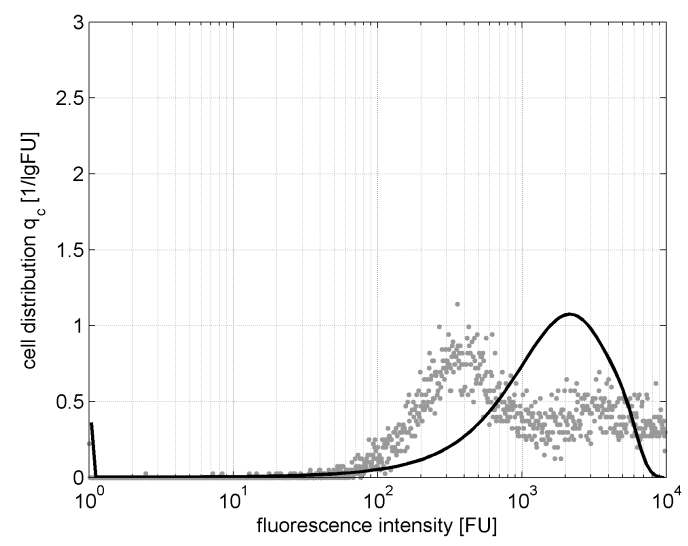

e) $t=66.17 \mathrm{~h}$ p.i.

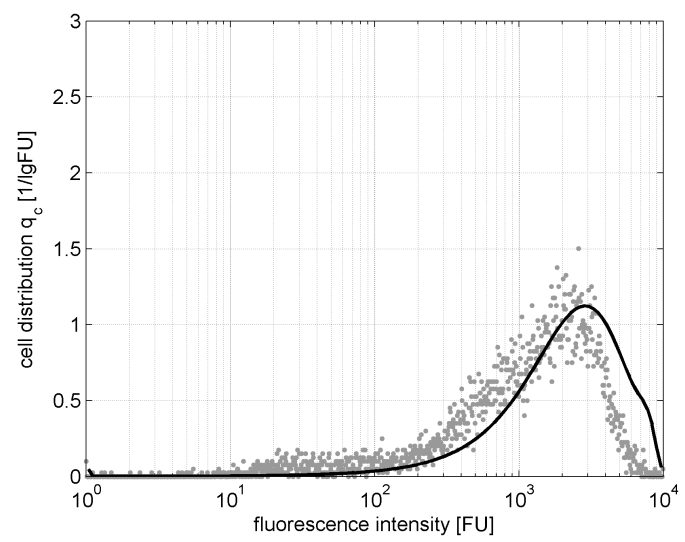

b) $t=34 \mathrm{~h}$ p.i.

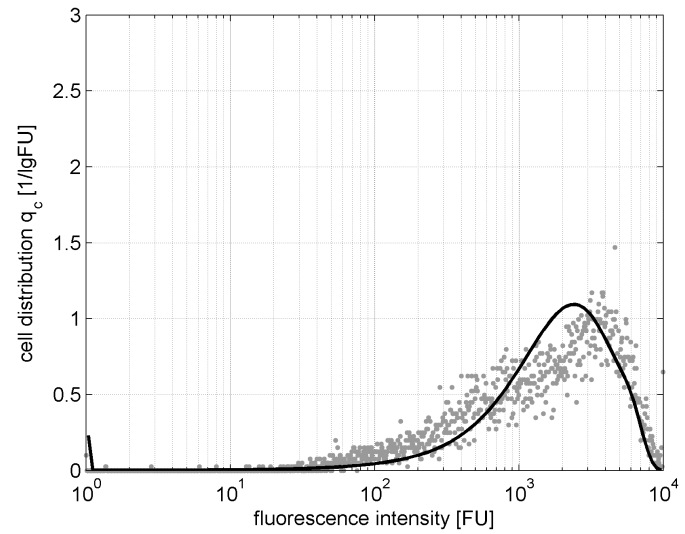

d) $t=54 \mathrm{~h}$ p.i.

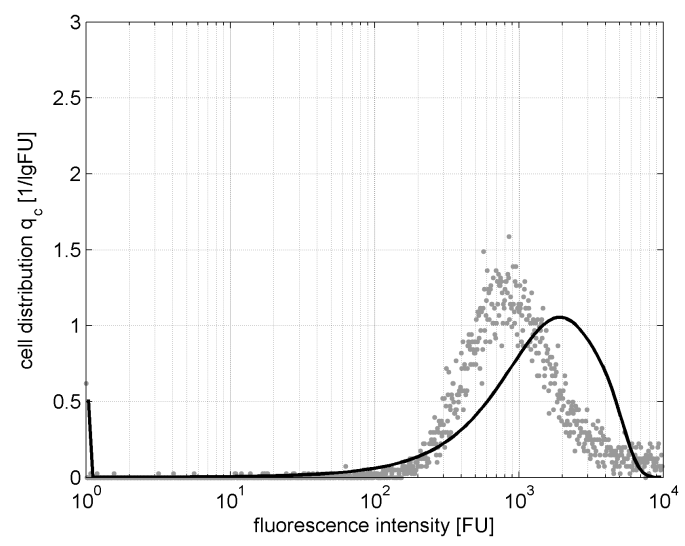

f) $t=78 \mathrm{hp}$.i.

Figure 33: Comparison of the distribution dynamics of the model of Müller et al. [2013] with experimental data of Schulze-Horsel et al. [2009] for A/H3N2, Experiment 1 (Part II) - simulated cell distributions are represented by lines; experimental cell distributions are represented by dots. 


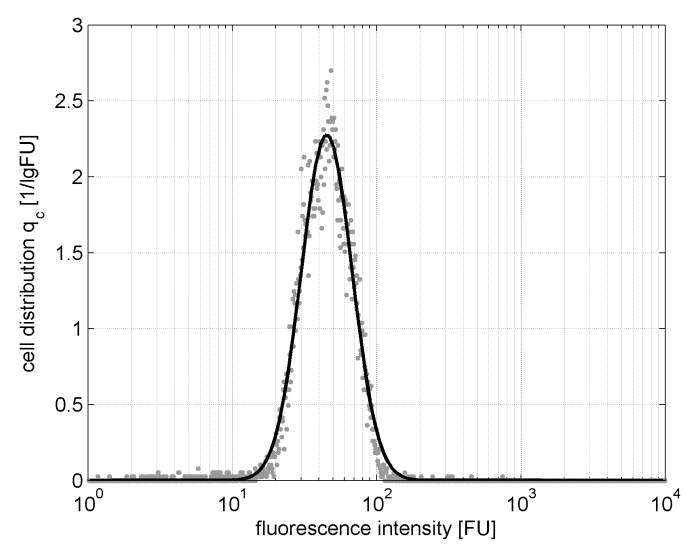

a) $t=0.25 \mathrm{~h} \mathrm{p.i.}$

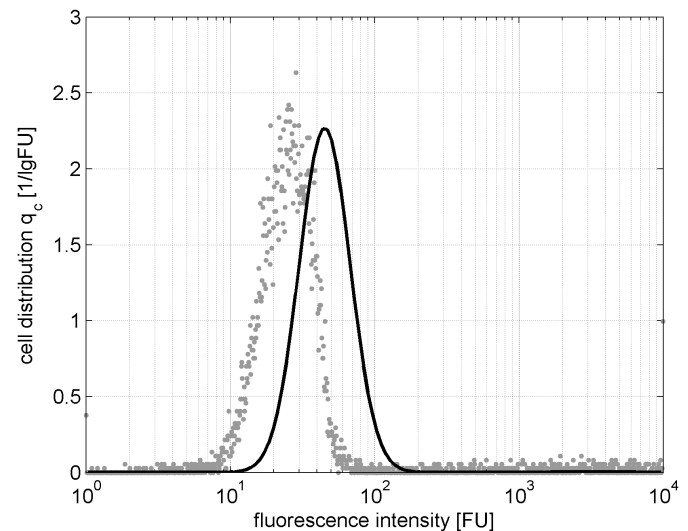

c) $t=10 \mathrm{~h}$ p.i.

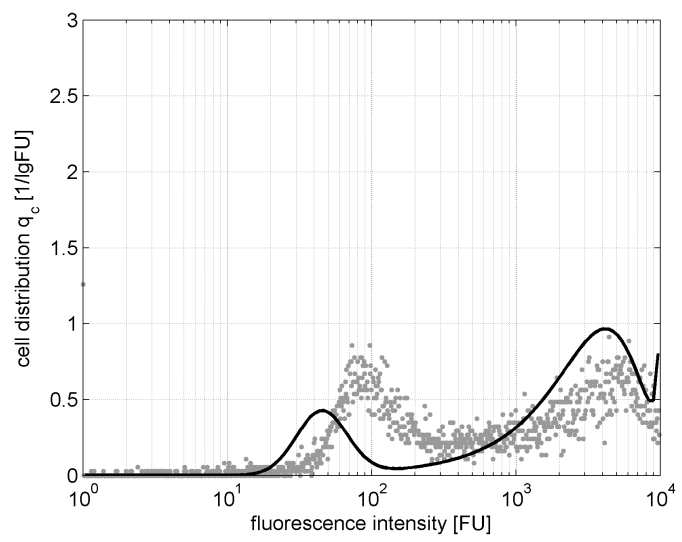

e) $t=18 \mathrm{hp}$.i.

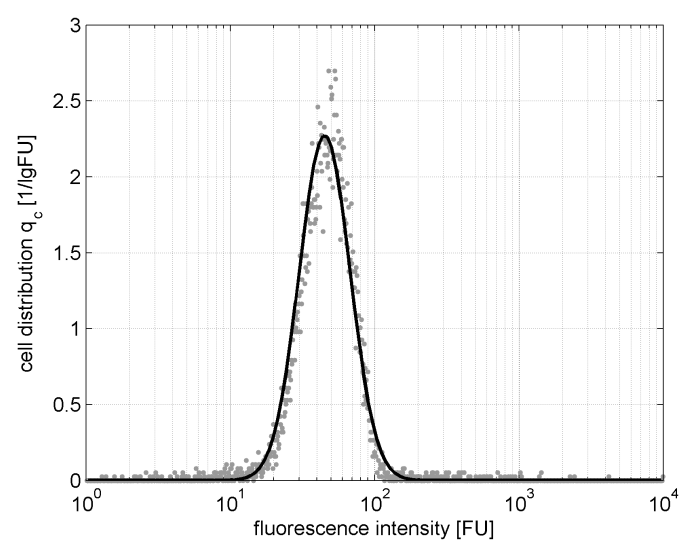

b) $t=6 \mathrm{~h}$ p.i.

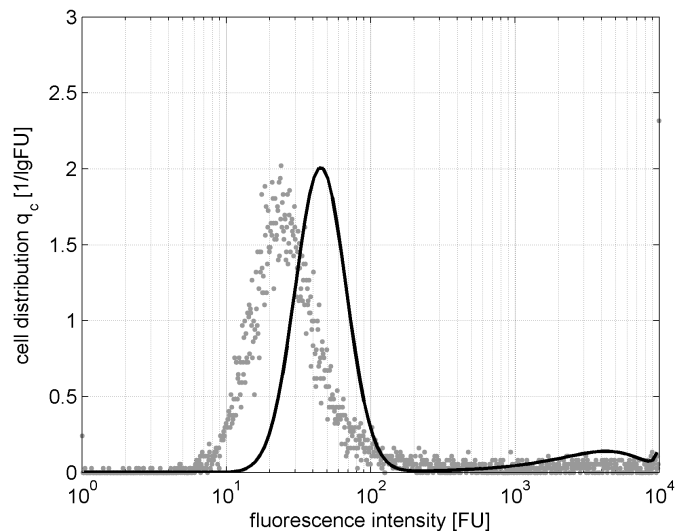

d) $t=14 \mathrm{~h}$ p.i.

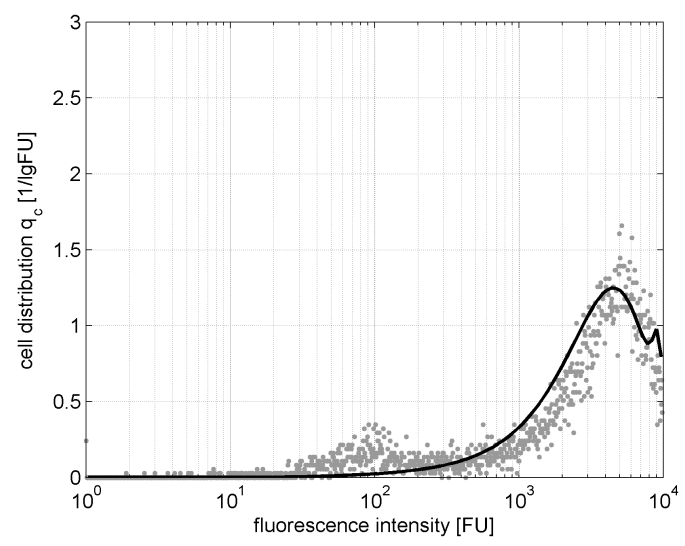

f) $t=22 \mathrm{~h} \mathrm{p.i.}$

Figure 34: Comparison of the distribution dynamics of the model of Müller et al. [2013] with experimental data of Schulze-Horsel et al. [2009] for A/H3N2, Experiment 2 (Part I) - simulated cell distributions are represented by lines; experimental cell distributions are represented by dots. 


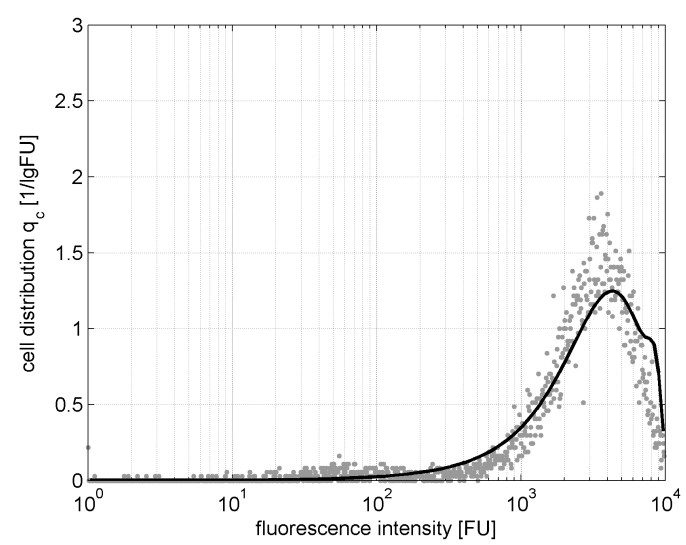

a) $t=26 \mathrm{~h}$ p.i.

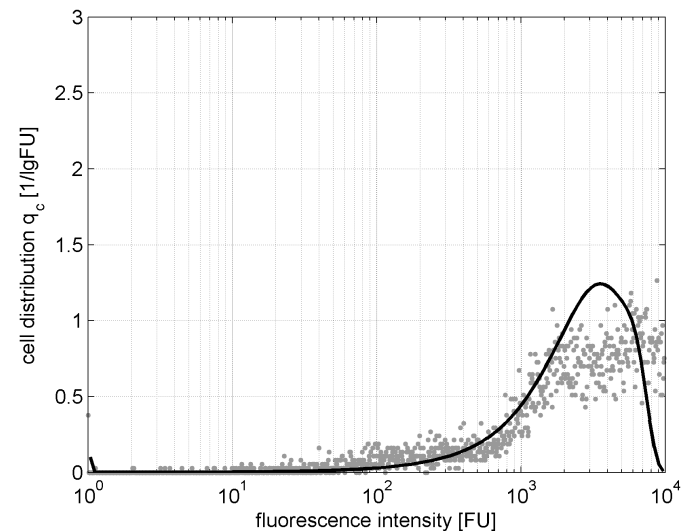

c) $t=42 \mathrm{~h}$ p.i.

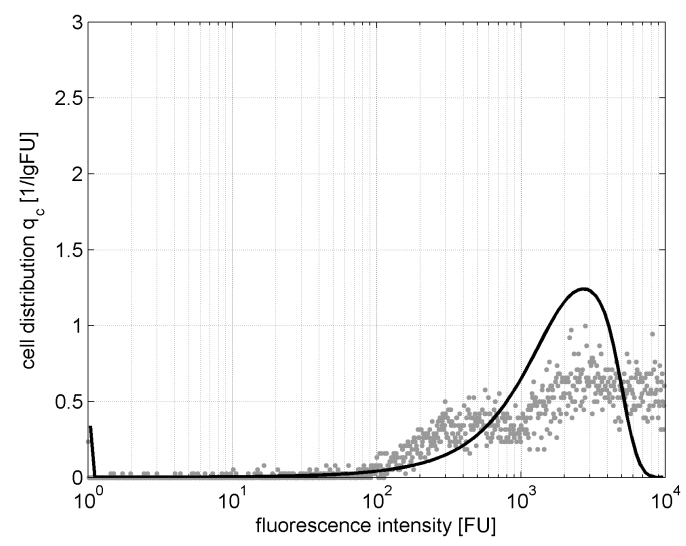

e) $t=66.17 \mathrm{~h}$ p.i.

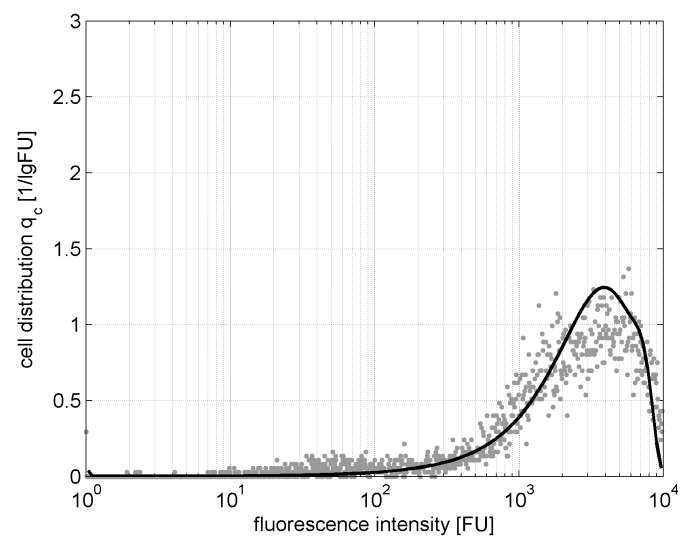

b) $t=34 \mathrm{~h}$ p.i.

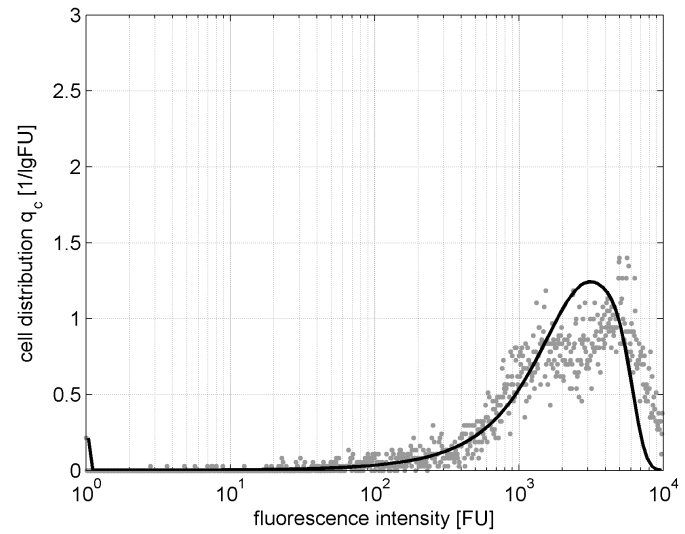

d) $t=54 \mathrm{~h}$ p.i.

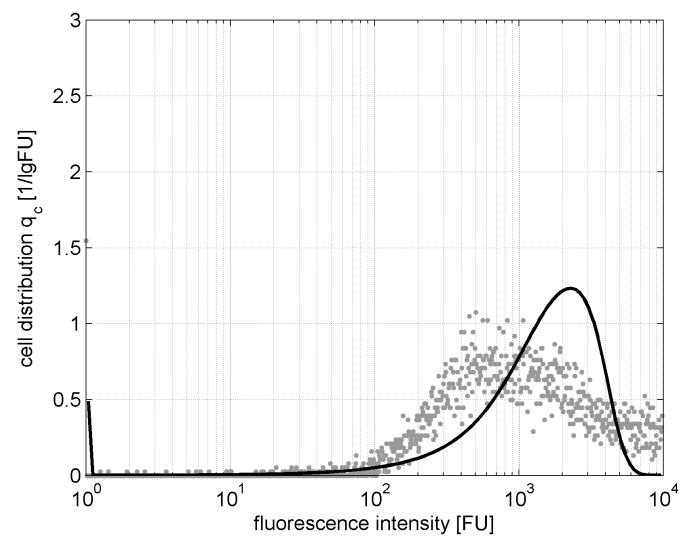

f) $t=78 \mathrm{hp}$.i.

Figure 35: Comparison of the distribution dynamics of the model of Müller et al. [2013] with experimental data of Schulze-Horsel et al. [2009] for A/H3N2, Experiment 2 (Part II) - simulated cell distributions are represented by lines; experimental cell distributions are represented by dots. 


\subsection{Special case A/H1N1-NIBSC}

As already mentioned, besides a high growth reassortant of $\mathrm{A} /$ Wisconsin/67/2005 (A/H3N2) Schulze-Horsel et al. [2009] also used two variants of A/Puerto Rico/8/34: A/H1N1-RKI and A/H1N1-NIBSC. Interestingly, the latter two, though nominally being the same strain, differ significantly in total virus yield and overall dynamics. In the case of A/H1N1-NIBSC virus replication occurs faster and apoptosis is induced earlier and stronger leading to a reduced virus yield [Schulze-Horsel et al., 2009]. This was further investigated by Seitz et al. [2010], Heynisch et al. [2010] and Vester et al. [2010]. They found out that A/H1N1-NIBSC is a generally stronger inducer of several signaling pathways belonging to the innate immune response, with particular emphasis on the IFN system, leading to a more intense stimulation of the cellular antiviral state. This directly translates into a distinct alteration of the distribution dynamics of the host cell population.

In Figs. 36 and 37 experimental cell distributions of the first experiment with A/H1N1NIBSC are presented. At $6.5 \mathrm{~h}$ p.i. a comparatively large fraction of cells has already accumulated significant amounts of viral NP. Note, that also the position of the left peak has moved to higher fluorescence intensities when compared to the initial distribution. This could either be caused by minimal viral protein production before an antiviral state is completely established, or it could be an artifact of the measurement procedure. However, the hallmark of this and the other experiment with A/H1N1-NIBSC is the extremely long perseverance of cells at rather low fluorescence intensities, presumably due to inhibition of successful replication by induction of an antiviral state. As an immune response and the respective alteration of the replication process are not considered in the model of Müller et al. [2013], difficulties arise to reproduce this behavior with biologically reasonable parameters. In the framework of the model an emulation of the described distribution dynamics would be possible by implying an increased transition to the second phase of replication, which is associated with the induction of apoptosis, and simultaneously boosting cell death at low degrees of fluorescence to prevent the cells from reaching the left boundary due to the related backshift. Though, not only does this seem biologically questionable. It would also be a rather poor substitute for a distinct consideration of an immune response of the host cell population of whatever kind.

When looking at the distribution dynamics of A/H1N1-RKI and A/H3N2 (Figs. 29 to 35 ), the cells virtually seem to change over from one subpopulation or, in the case of the model, from one species to another in a more or less predefined sequence: from uninfected cells to infected cells and eventually apoptotic cells. As soon as all cells are infected and productive this virtually results in a homogeneous cell distribution, which exclusively consists of apoptotic cells at later times post infection. The results with A/H1N1-NIBSC suggest that, in order to reproduce the experiments, there must be 


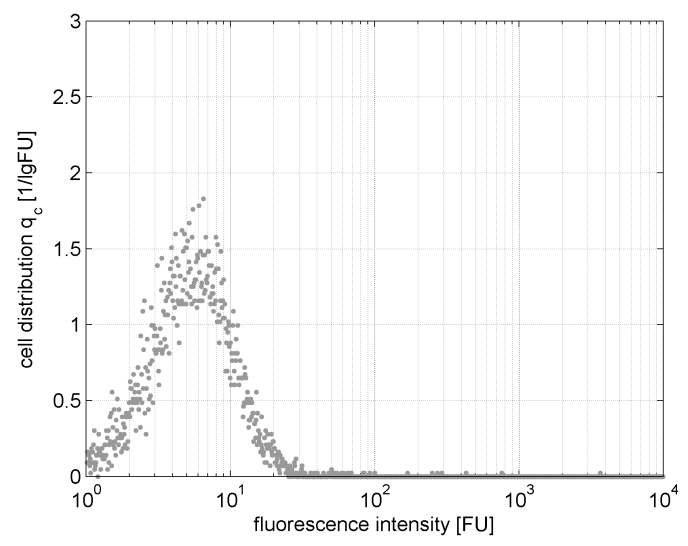

a) $t=0.5 \mathrm{~h}$ p.i.

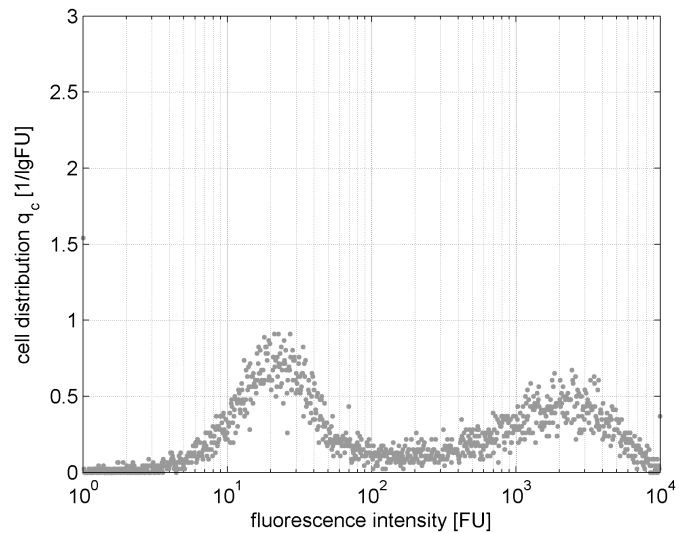

c) $t=10.5 \mathrm{~h}$ p.i.

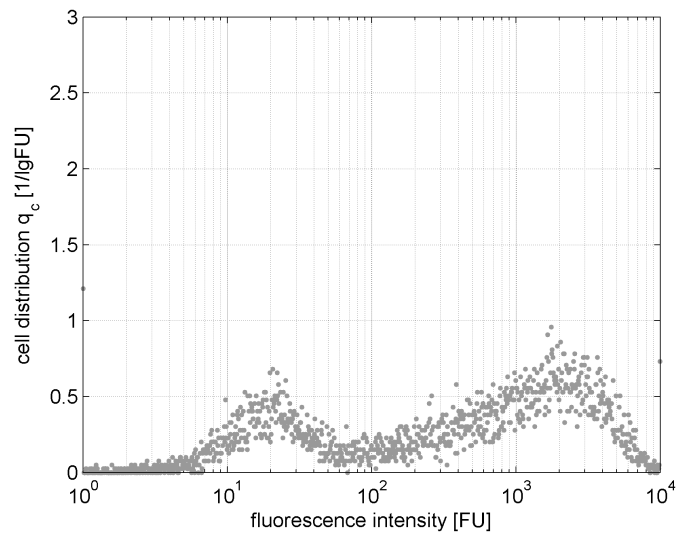

e) $t=18.5 \mathrm{~h}$ p.i.

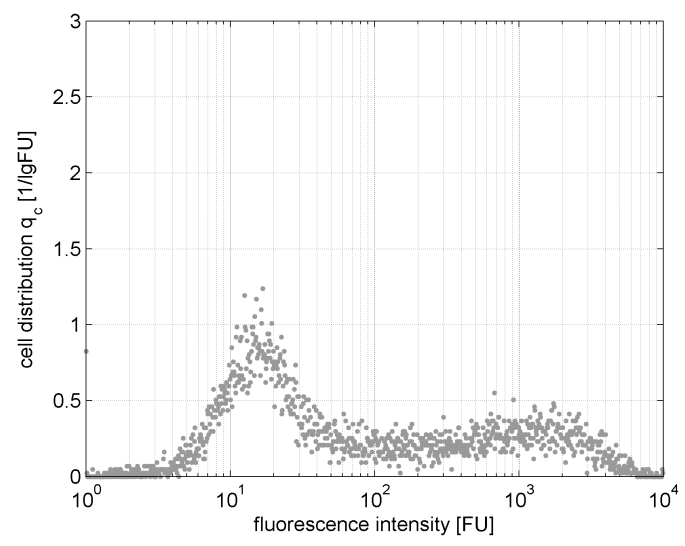

b) $t=6.5 \mathrm{~h}$ p.i.

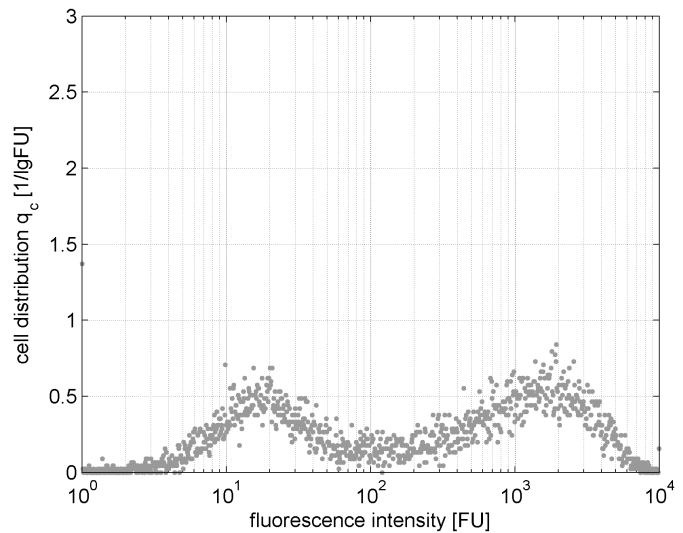

d) $t=14.5 \mathrm{~h} \mathrm{p.i.}$

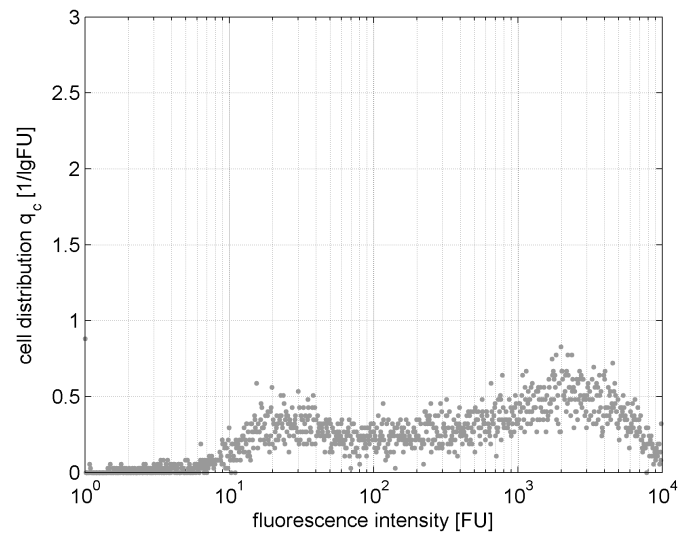

f) $t=26.5 \mathrm{~h}$ p.i.

Figure 36: Experimental distribution dynamics for A/H1N1-NIBSC, Experiment 1 (Part I) [SchulzeHorsel et al., 2009]. 


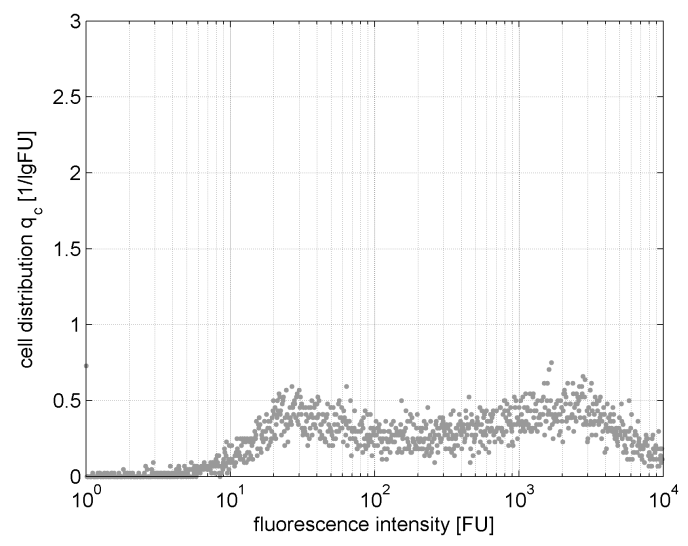

a) $t=30.5 \mathrm{~h} \mathrm{p.i.}$

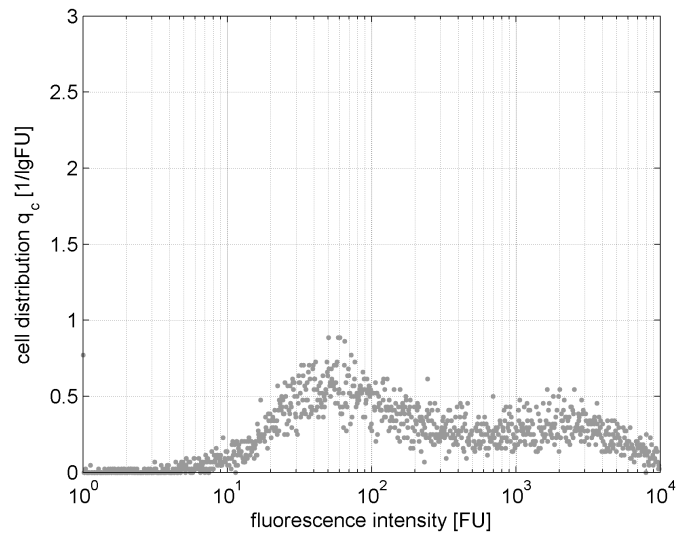

c) $t=38.5 \mathrm{~h} \mathrm{p.i.}$

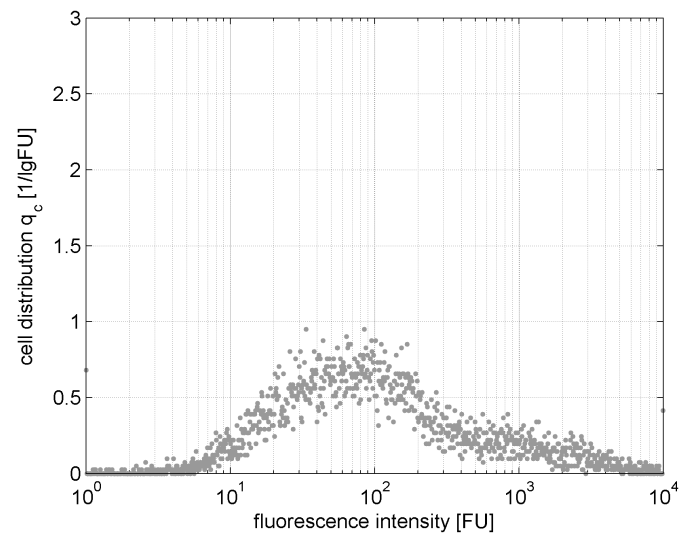

e) $t=50.5 \mathrm{~h} \mathrm{p.i.}$

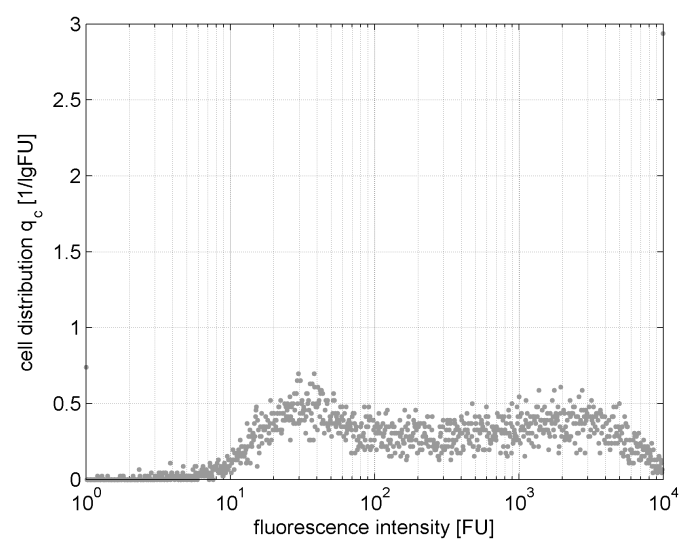

b) $t=34.5 \mathrm{~h}$ p.i.

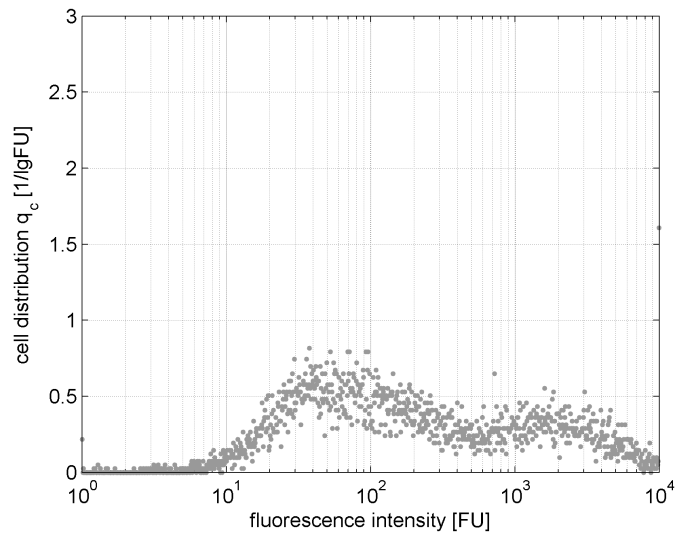

d) $t=42.5 \mathrm{~h} \mathrm{p.i.}$

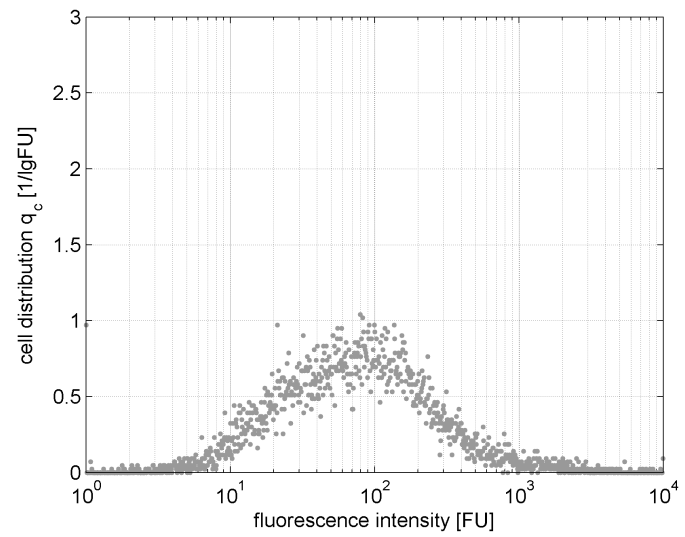

f) $t=72.5 \mathrm{~h}$ p.i.

Figure 37: Experimental distribution dynamics for A/H1N1-NIBSC, Experiment 1 (Part II) [SchulzeHorsel et al., 2009]. 
at least one more subpopulation, namely, cells in the antiviral state, which eventually also turn apoptotic. The second experiment with A/H1N1-NIBSC (Figs. 38 and 39) further underlines the necessity of this estimation. Furthermore, a peculiar phenomenon is revealed which sets the second experiment with A/H1N1-NIBSC apart from previous results and also impedes a plausible reproduction by the present model. From $26 \mathrm{~h}$ p.i. on a third peak is formed as a result of proceeding apoptosis of cells with both high and low intracellular content of viral NP, leading to a trimodal cell distribution, which is slowly resolved at later times, when eventually all cells have turned apoptotic.

The comparison of the experimental results for all three considered viruses underlines the importance of properly adapted virus strains for the influenza A vaccine production. With exception of the HA and the NA gene A/H1N1-RKI and A/H3N2 share the same genome $-\mathrm{A} / \mathrm{H} 3 \mathrm{~N} 2$ is a high growth reassortant with a A/Puerto Rico/8/34 backbone. Hence, both virus strains are characterized by high yields and a relatively small number of apoptotic cells. Only a "minor" alteration of the NS1 gene sets A/H1N1-NIBSC apart from the others [Seitz et al., 2010] and renders it as rather unfavorable for vaccine production. Accordingly, A/H1N1-NIBSC takes a distinguished position among the considered virus strains and its case nicely illustrates the need for further experimental and theoretical investigation of the influenza vaccine production process. 


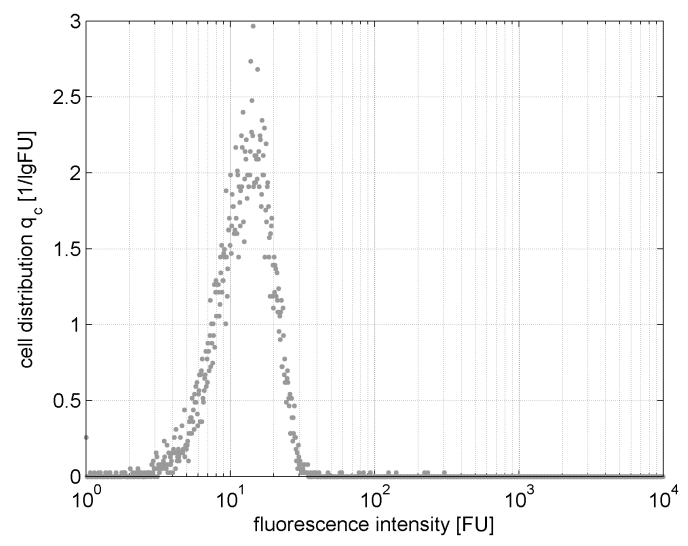

a) $t=0.25 \mathrm{hp}$.i.

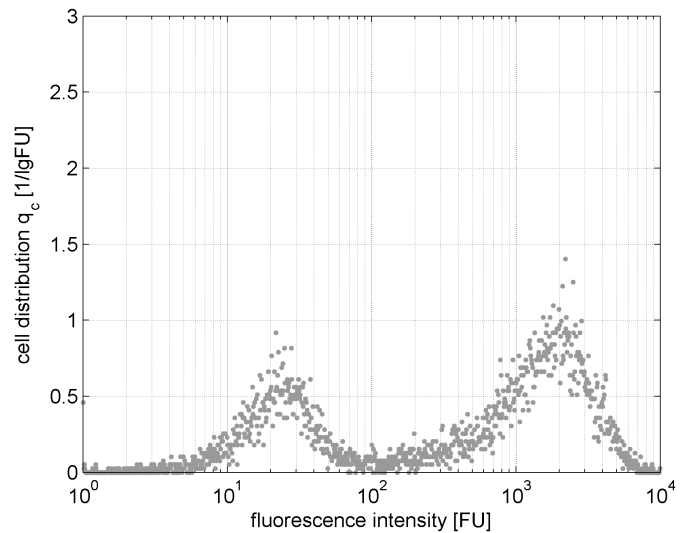

c) $t=10 \mathrm{~h}$ p.i.

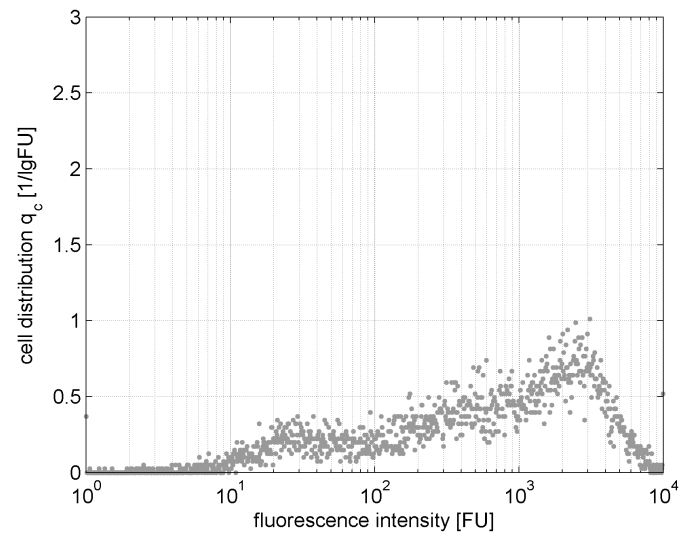

e) $t=18 \mathrm{~h}$ p.i.

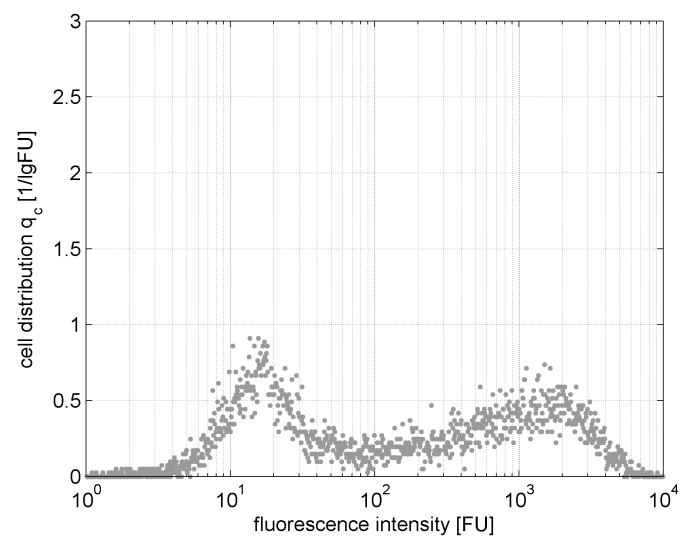

b) $t=6 \mathrm{~h}$ p.i.

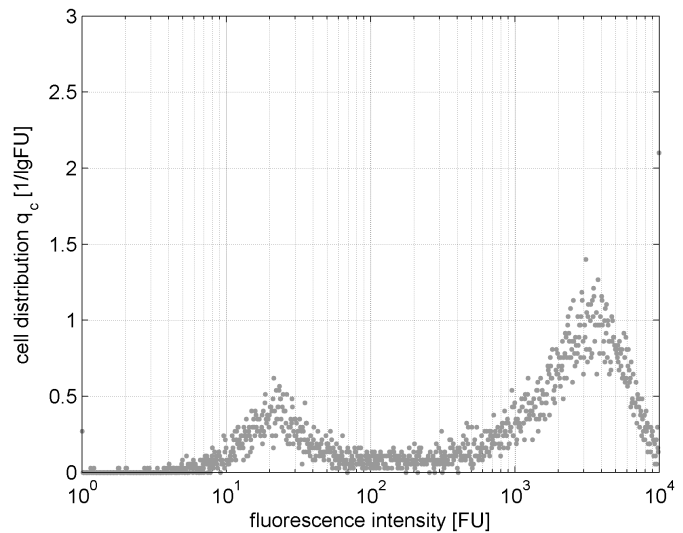

d) $t=14 \mathrm{~h}$ p.i.

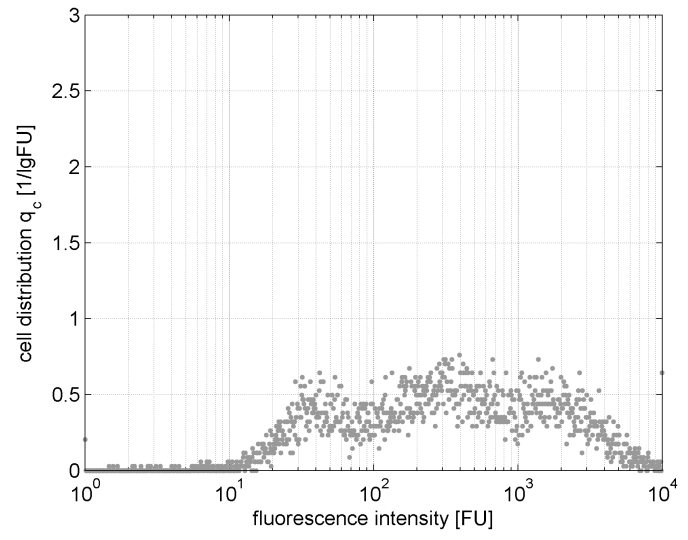

f) $t=26 \mathrm{~h} \mathrm{p.i.}$

Figure 38: Experimental distribution dynamics for A/H1N1-NIBSC, Experiment 2 (Part I) [SchulzeHorsel et al., 2009]. 


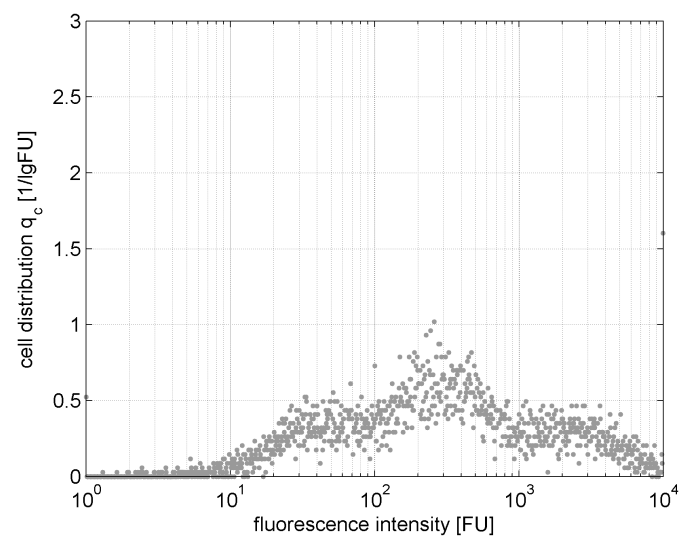

a) $t=30 \mathrm{~h}$ p.i.

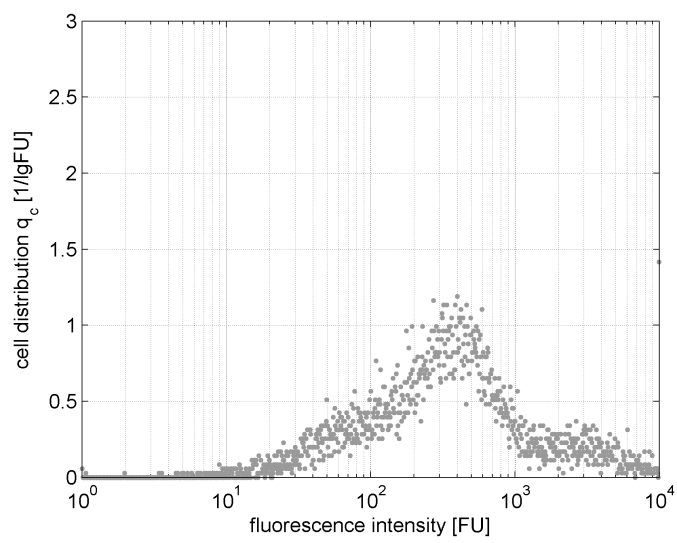

c) $t=38 \mathrm{~h}$ p.i.

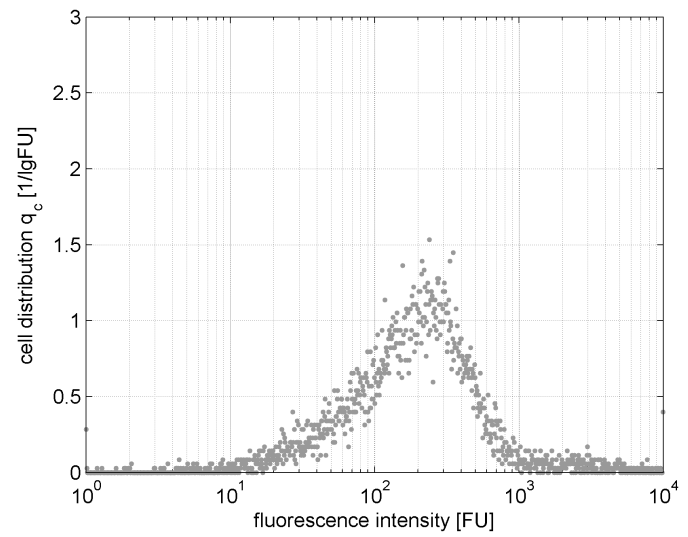

e) $t=49.5 \mathrm{~h} \mathrm{p.i.}$

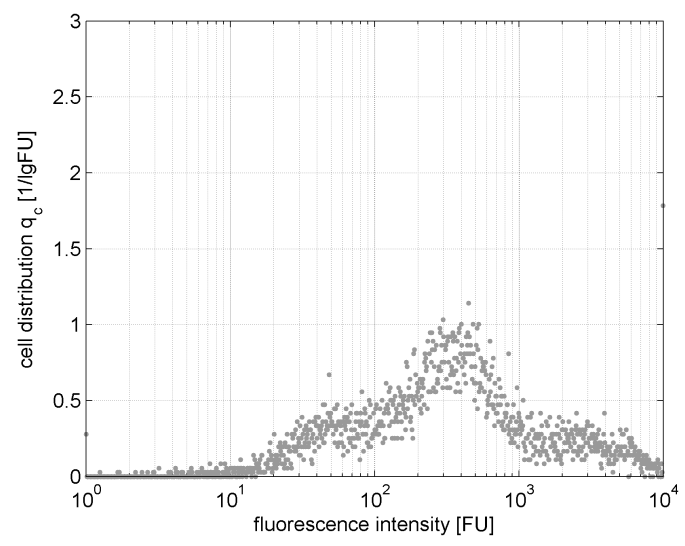

b) $t=34 \mathrm{~h}$ p.i.

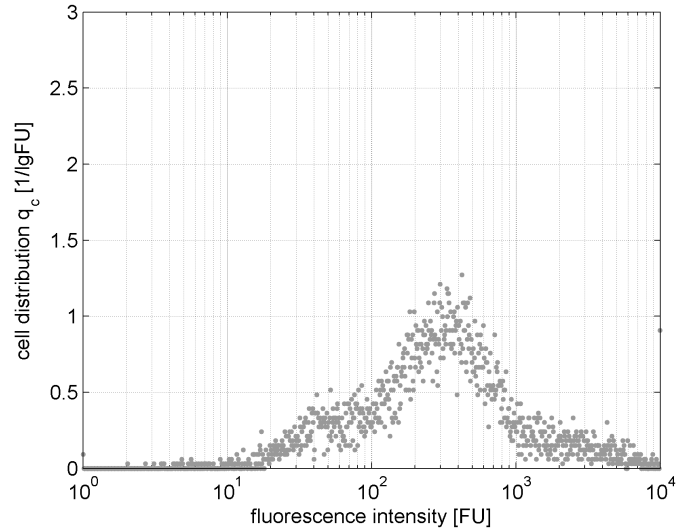

d) $t=42 \mathrm{~h}$.i.

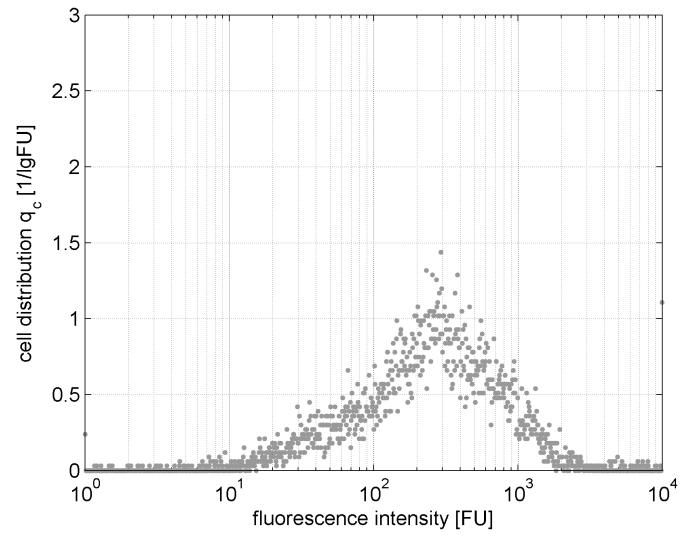

f) $t=72.5 \mathrm{~h}$ p.i.

Figure 39: Experimental distribution dynamics for A/H1N1-NIBSC, Experiment 2 (Part II) [SchulzeHorsel et al., 2009]. 


\section{Conclusion}

\subsection{Summary}

In the preceding sections the development and evolution of a deterministic population balance model of the influenza A virus infection of adherent MDCK cells has been presented. From the basic distributed model [Müller et al., 2008] based on the lumped approach by Möhler et al. [2005] to the latest installment by Müller et al. [2013] every step of development was driven by the ambition to enhance the existing model structure in order to refine the general understanding of the underlying process with regard to the available experimental information.

In the first distributed model by Müller et al. [2008] the degree of fluorescence was introduced as an internal coordinate to directly incorporate flow cytometric data collected by Schulze-Horsel et al. [2008] for the infection of adherent MDCK cell cultures with equine influenza A virus (see Section 3). The degree of fluorescence is linked to the intracellular amount of viral protein and its temporal change constitutes a gauge for the progress of infection. The most significant feature of the experimental cell distribution dynamics is the non-monotonous progression along the fluorescence intensity with the typical reversal of the propagation direction at later times post infection. Although other characteristic phenomena, e.g. transient multimodality, are also present in the experimental data of equine influenza A virus (see Figs. 15 to 20, page $36 \mathrm{ff}$.), they are not as pronounced as in the case of the human influenza A virus strains Schulze-Horsel et al. [2009] examined later. In addition, in contrast to Sections 3 and 4, before the publication of the model of Müller et al. [2013] the latent phase was approximated by simply shifting the simulation results for a certain amount of time $\left(t_{\text {shift }}\right)$ [Müller et al., 2008, 2011], as already performed before by Möhler et al. [2005]. Thus, experimental data between the time of infection and $t_{\text {shift }}$ have not been considered back when the model of Müller et al. [2008] was published. Accordingly, at that time highest priority was given to the further development of the existing model framework with the aim of reproducing the so called backshift.

Though never officially published, many different approaches have been proposed to reproduce the backshift. Finally, the idea to split up the virus replication process into two phases provided the most promising candidate, which proved to be the stepping stone for future model approaches when more substantial experimental data were available (see Section 4). Hence, together with the experimental work concerning different human influenza A virus strains published by Schulze-Horsel et al. [2009] and Schulze-Horsel [2011] the "two-phase model" established the basis for what would become the population balance model of Müller et al. [2013] (see Section 5). 
The main hypothesis, which formed in the light of the new experimental data, was, that the transition from one replication phase to the other is eventually triggered by the induction of apoptosis. Thus, with respect to the model, cells in the second phase of replication are considered to be in an early state of apoptosis, which is characterized by altered distribution dynamics. Unfortunately, the experimental data turned out to be unsuitable to further elaborate this hypothesis, because Schulze-Horsel et al. focused on the apoptotic fragmentation of host cell DNA, which is too late an event in the course of apoptosis to actually be responsible for the backshift of the cell distribution. Hence, as the hypothesis could not be substantiated yet, it stays debatable whether cells in the second phase of replication are apoptotic or not and, if the former is true, in which phase of induction of apoptosis they are. Alternatively, a less biased terminology, as applied in Section 4, could have been adopted, but eventually was given up in order to stay in accordance with the original publication of Müller et al. [2013]. However, with respect to the current knowledge a significant influence of apoptosis on the overall process of vaccine production can be taken for granted, but in the end, only further experimental research that investigates the induction or very early events of apoptosis during influenza A virus infection can help to clarify the matter.

As mentioned above, the model of Müller et al. [2013] actually constitutes the first instance in which the latent phase is implemented in a direct manner. In this way, distinct waves of virus release can be produced, which are characteristic for the early phase of vaccine production processes with low multiplicities of infection. In addition, delicate distribution dynamics comprising transient multimodalities can be addressed more directly. In summary, the model is able to reproduce all characteristic dynamic phenomena of the considered process at least for two of the examined virus strains. Of course not every trait is covered in full detail and there are weaknesses and drawbacks concerning certain model assumptions. But this should come as no surprise when one compares the relative simplicity of the model with the complexity of the underlying process. As the example of A/H1N1-NIBSC shows, there is always a need for thorough experimental investigation to reveal certain aspects that otherwise could have been overlooked, e.g. the importance of the immune response.

In general, the comparison of simulation results with respective experimental data at best provides correspondence between the two and meets the desired expectations. But more often existing discrepancies are exposed, which eventually proves to be even more fruitful, as the ambition to resolve these discrepancies constitutes the driving force that stimulates a reconsideration of the model assumptions and an improvement of the model structure, which, in the end, promotes further progress. Hence, in combination with meaningful experimental data mathematical models provide an essential contribution to the understanding of the underlying process. Accordingly, the presented approach constitutes a useful framework for the deterministic population balance modeling of the influenza vaccine production and its improvement should be further promoted. 


\subsection{Outlook}

In order to pick up on the last proposal some suggestions on how to improve certain aspects of the model are presented in the following.

\subsubsection{Continuous alteration of the replication dynamics}

In order to allow a smoother transition from one replication phase to another, instead of the switching behavior implemented in the model, a further differentiation of the infected cells seems to be required. This would result in a two-dimensional cell population and points in a possible direction for the development of deterministic population balance models, namely, to define significant state variables on the single cell level, which can be translated into significant internal coordinates on the population level. For instance, in accordance with the hypothesis of the induction of apoptosis being responsible for the altered replication dynamics one could think of an additional internal coordinate $\alpha$, which is determined by the state of the infected cells with respect to their progress within the apoptotic cascade. Therefore, characteristic positions or states within the apoptotic network have to be defined and methods to detect these states have to be established. Under consideration of the two-dimensional distribution of the cell population a very simple implementation of these thoughts could be formulated for the infected cells:

$$
\begin{aligned}
\frac{\partial \breve{\widetilde{I}}_{\mathrm{c}}(t, \varphi, \alpha)}{\partial t}= & k_{\mathrm{vi}} \breve{\widetilde{U}}_{\mathrm{c}}(t-\tau, \varphi, \alpha) V_{\mathrm{ac}}(t-\tau)-k_{\mathrm{cd}}(\varphi, \alpha) \breve{\widetilde{I}}_{\mathrm{c}}(t, \varphi, \alpha) \\
& -\frac{\partial}{\partial \varphi}\left(k_{\mathrm{net}}(\varphi, \alpha) \breve{\widetilde{I}}_{\mathrm{c}}(t, \varphi, \alpha)\right)-\frac{\partial}{\partial \alpha}\left(k_{\mathrm{apo}}(\varphi, \alpha) \breve{\widetilde{I}}_{\mathrm{c}}(t, \varphi, \alpha)\right) .
\end{aligned}
$$

In the equation above infection of uninfected cells is handled in a similar fashion as in Eq. (5.3) on page 58. Progress along $\alpha$ is determined by the apoptosis coefficient $k_{\text {apo }}$ which may depend on both internal coordinates $\varphi$ and $\alpha$. Likewise, the net coefficient $k_{\text {net }}$ and the cell death rate $k_{\mathrm{cd}}$ may be functions of $\varphi$ and $\alpha$. In this way the cell population is able to move within the two-dimensional space defined by the two internal coordinates. Thus, a separate population of apoptotic cells is not required anymore.

In fact, after the work of Schulze-Horsel et al. [2009] had been published, attempts have been made to adapt a similar approach to two-dimensional flow cytometric data with the intracellular amount of viral NP constituting one dimension and the accumulation of DNA strand breaks serving as the other dimension. In Figure 40 examples of flow cytometric data of the first experiment with A/H3N2 are given for selected times post infection. These examples show that in the beginning the fluorescence intensity is low in both dimensions. Due to NP production of infected cells the latter move towards the 


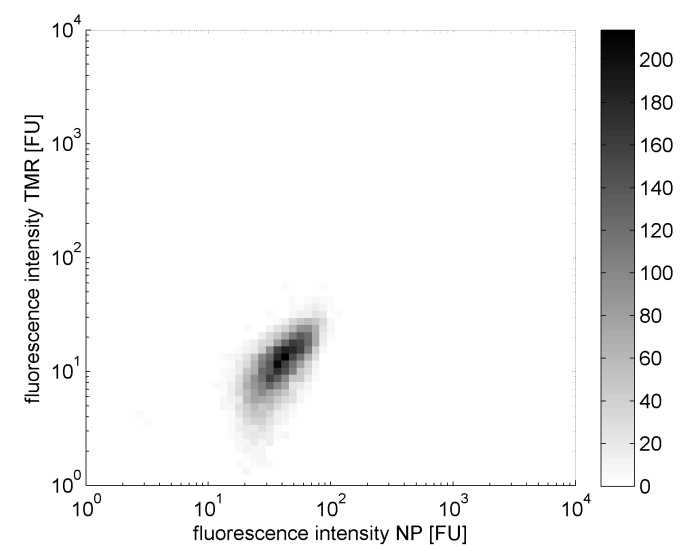

a) $t=0.25 \mathrm{~h}$ p.i.

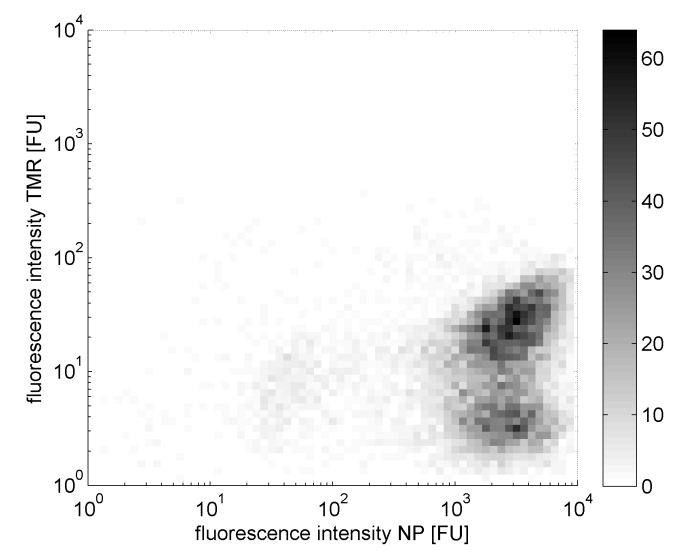

c) $t=26 \mathrm{~h}$ p.i.

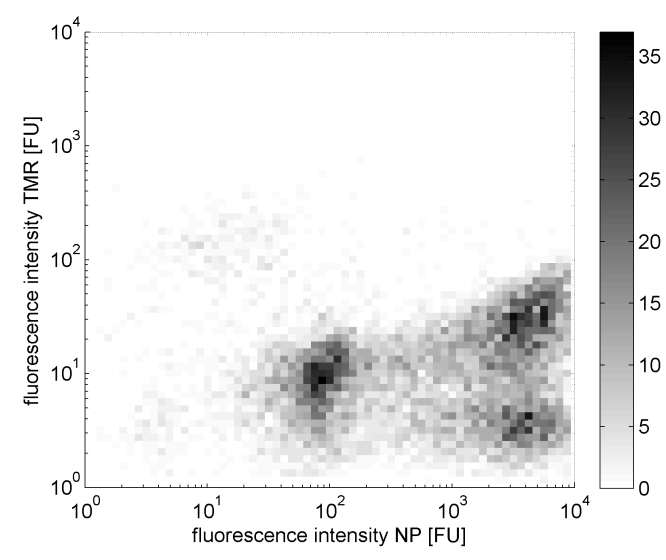

b) $t=18 \mathrm{~h}$ p.i.

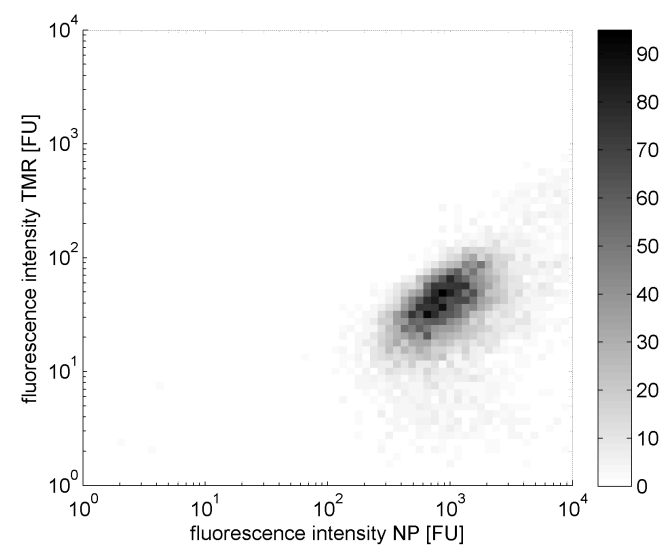

d) $t=78 \mathrm{~h}$ p.i.

Figure 40: Flow cytometric data, raw cell counts for selected times p.i. for A/H3N2, Experiment 1 [Schulze-Horsel et al., 2009] (cell counts of 16 consecutive sensor channels are combined to form one "pixel") - ordinate: fluorescence intensity due to viral NP, abscissa: fluorescence intensity due to DNA strand breaks

right side of the plane (18h p.i.). A slight upward movement may already indicate a slow accumulation of DNA strand breaks. At $26 \mathrm{~h}$ p.i. nearly all cells can be found in the lower right quadrant of the fluorescence intensity plane. Though, the reason for the noticeable formation of two subpopulations with similar NP content is not clear as both subpopulations do not differ in their subsequent behavior with respect to NP content. However, later in the experiment more or less all cells are united again in one population that shows lower fluorescence intensity due to viral NP, but higher fluorescence intensity due to DNA strand breaks (78 h p.i.).

Although these results look promising, the differentiation of cells according to their accumulated number of DNA strand breaks turns out to be of only minor significance 
as far as the reversal of the propagation direction is concerned. That is because when DNA fragmentation is finally induced respective cells are already in a very late state of apoptosis where virus replication eventually stops. Unfortunately, with regard to the present model formulation the transient towards this state of interest is not resolved. This underlines the great importance of a suitable definition and meaningful detection of various characteristic states between the induction of apoptosis and the eventual DNA fragmentation in order to facilitate a significant differentiation of the cell population.

\subsubsection{Distinction between the eclipse phase and the latent phase}

As mentioned before, in the presented model it is assumed that the eclipse phase and the latent phase coincide. Hence, when fitting the model to experimental data a tradeoff between the integral dynamics and the distribution dynamics is made. In order to resolve this trade-off and simultaneously improve the biological plausibility of the model a distinction between the two phases is recommendable. With respect to the model the eclipse phase is considered to incorporate all processes between the successful infection of a host cell and the initial production of viral NP. Furthermore, the latent phase comprises all processes from the infection to the release of the first free virus particles. The durations of the eclipse phase and the latent phase shall be defined as $\tau_{1}$ and $\tau_{2}$, respectively. Their difference shall be defined as $\tau_{2-1}$, yielding

$$
\tau_{1} \in[0, \infty] \quad \text { and } \quad \tau_{2} \geq \tau_{1} \quad \text { and } \quad \tau_{2-1}=\tau_{2}-\tau_{1}
$$

Hence, the change of the number density of the infected cells that have passed the eclipse phase (former Eq. (5.3)) accounts to

$$
\frac{\partial \tilde{I}_{c}(t, \varphi)}{\partial t}=k_{\mathrm{vi}} \tilde{U}_{c}\left(t-\tau_{1}, \varphi\right) V_{\mathrm{ac}}\left(t-\tau_{1}\right)-\frac{\partial}{\partial \varphi}\left(k_{\mathrm{net}}(\varphi) \tilde{I}_{c}(t, \varphi)\right)-k_{\mathrm{apo}}(\varphi) \tilde{I}_{c}(t, \varphi) .
$$

After the eclipse phase has been traversed infected cells start to change their degree of fluorescence by means of viral NP production and RNP complex formation. Nevertheless, free virus particles are not released before the latent phase has been passed. Eqs. (5.5) 
and (5.6) have to be adapted accordingly:

$$
\begin{aligned}
\frac{\mathrm{d} V_{\mathrm{ac}}(t)}{\mathrm{d} t}= & P_{\mathrm{eff}} k_{\mathrm{rel}} \int_{\varphi}\left(\tilde{I}_{c}\left(t-\tau_{2-1}, \varphi\right)+\tilde{A}_{c}\left(t-\tau_{2-1}, \varphi\right)\right) \mathrm{d} \varphi \\
& -k_{\mathrm{vi}} V_{\mathrm{ac}} \int_{\varphi} \tilde{U}_{c}(t, \varphi) \mathrm{d} \varphi-k_{\mathrm{deg}} V_{\mathrm{ac}}(t), \\
\frac{\mathrm{d} V_{\mathrm{inac}}(t)}{\mathrm{d} t}= & \left(1-P_{\mathrm{eff}}\right) k_{\mathrm{rel}} \int_{\varphi}\left(\tilde{I}_{c}\left(t-\tau_{2-1}, \varphi\right)+\tilde{A}_{c}\left(t-\tau_{2-1}, \varphi\right)\right) \mathrm{d} \varphi+k_{\mathrm{deg}} V_{\mathrm{ac}}(t) .
\end{aligned}
$$

A schematic depiction of the resulting model is presented in Fig. 41.

\subsubsection{Multiple infections}

The genome of influenza A virus comprises eight individual RNA segments. When multiple influenza A virus particles infect the same host cell their possibly varying genomes are replicated equally, resulting in the release of virus particles with all kinds of arbitrary combinations of the original RNA segments provided by the infecting virus particles. This ability of influenza A virus is called antigenic shift. Together with the so called antigenic drift, i.e. the tendency of influenza A virus to change its antigenic properties by means of mutation, antigenic shift represents the most significant asset that affects the genetic variability of the virus. For this process to happen multiple infections are a basic prerequisite and should therefore not be neglected in a model of the process.

Accordingly, free virus particles should not only be allowed to attach to uninfected cells but to any cell. If the basic distinction between active and inactive virus particles is maintained, in the simplest case active virus particles are assumed to attach to any cell with equal affinity and inactive virus particles are assumed to stay passive. Hence, Eq. (5.5) is changed to

$$
\begin{aligned}
& \frac{\mathrm{d} V_{\mathrm{ac}}(t)}{\mathrm{d} t}= P_{\mathrm{eff}} k_{\mathrm{rel}} \int_{\varphi}\left(\tilde{I}_{c}(t, \varphi)+\tilde{A}_{c}(t, \varphi)\right) \mathrm{d} \varphi \\
& \quad-k_{\mathrm{va}} V_{\mathrm{ac}}(t) \int_{\varphi} \tilde{X}_{c}(t, \varphi) \mathrm{d} \varphi-k_{\mathrm{deg}} V_{\mathrm{ac}}(t) \\
& \text { with } \quad \tilde{X}_{c}(t, \varphi)=\tilde{U}_{c}(t, \varphi)+\tilde{L}_{c}(t, \varphi)+\tilde{I}_{c}(t, \varphi)+\tilde{A}_{c}(t, \varphi) .
\end{aligned}
$$

Here, $\tilde{X}_{c}(t, \varphi)$ denotes the sum of the number densities of all cells. The attachment of active virus particles to these cells is characterized by the attachment coefficient $k_{\mathrm{va}}$. If 


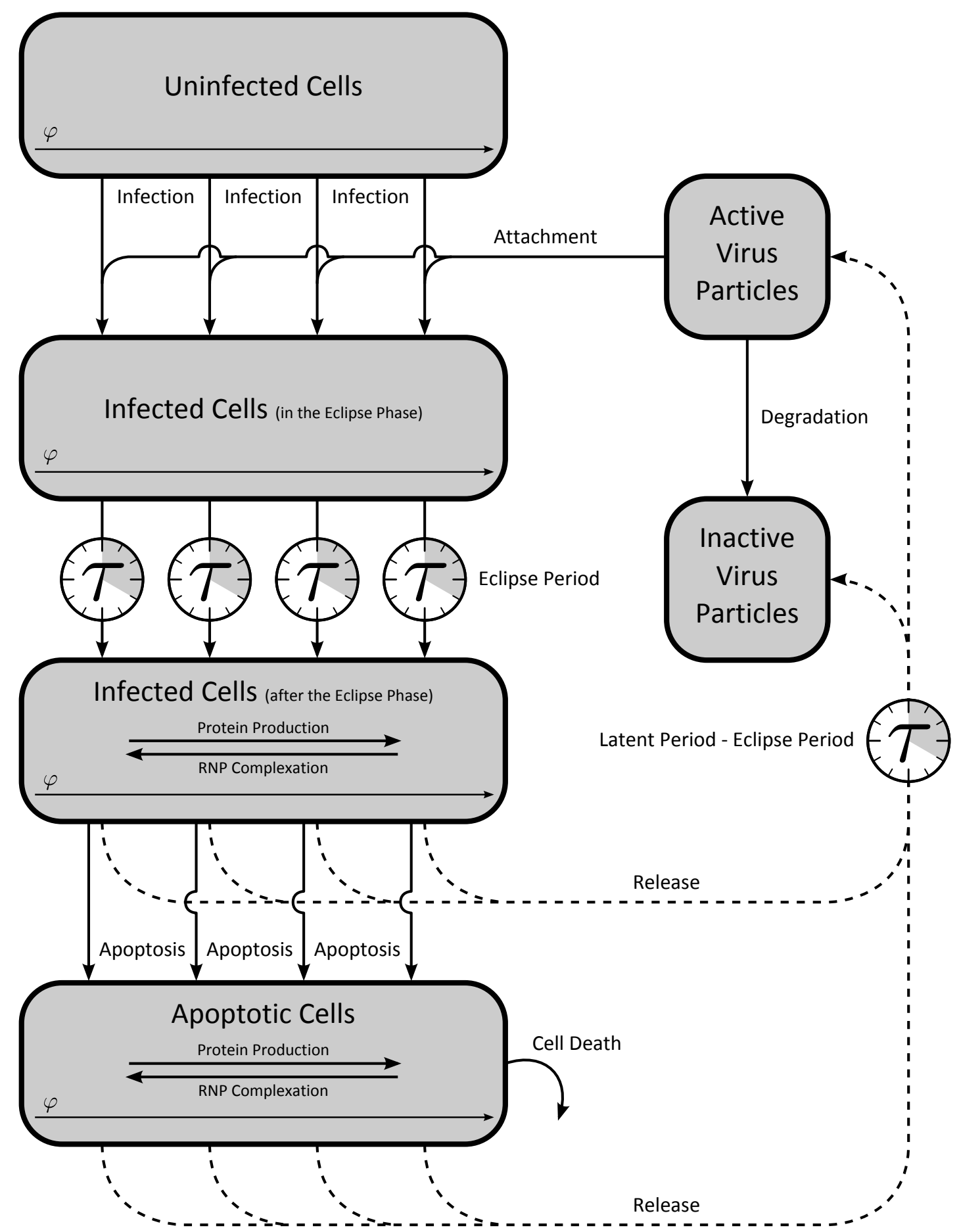

Figure 41: Scheme of a proposed model in consideration of the distinction between the eclipse phase and the latent phase. After the eclipse phase infected cells start to produce viral NP and form RNP complexes. Subsequently, free virus particles are released by infected and apoptotic cells after the remaining difference between the latent phase and the eclipse phase has been passed. 
every event of attachment of an active virus particle leads to a successful infection of the host cell, as it has been assumed in the model of Müller et al. [2013], $k_{\mathrm{va}}$ equals the infection coefficient $k_{\mathrm{vi}}$. Respectively, if the possibility of an incomplete infection despite successful attachment should be considered, $k_{\mathrm{va}}$ has to be larger than $k_{\mathrm{vi}}$.

This approach of tackling multiple infections is very straightforward. Nevertheless, it can form the basis for more intricate variants that incorporate more specific considerations. Possible questions that arise during the implementation of multiple infections are, for example: Does the affinity with which active virus particles attach to cells depend on the state of the host cell? Is the number of susceptible binding cites a limiting factor? Does the detachment of an already attached virus particle have to be considered? How important is the number of available endosomes? Does the uptake of multiple virus particles affect the replication dynamics and the performance of the host cell, and is it therefore useful to discriminate between cells with different numbers of absorbed virus particles? If the number of infecting virus particles has an effect on the replication dynamics, does the eclipse phase have to be considered separately for each virus particle that is taken up? These questions shall give an impression of the potential complexity of multiple infections.

In summary, multiple infections are an integral part of influenza virus replication and should therefore be taken into account in one way or another. This is underlined in the next section, as multiple infections are also a necessary prerequisite for defective virus particles to interfere with standard virus particles.

\subsubsection{Defective interfering particles}

Defective interfering particles (DIPs) are part of the family of biologically active influenza virus particles [Marcus et al., 2009]. As Frensing et al. [2013] show, their influence becomes especially apparent in continuous vaccine production processes. However, in conventional batch processes DIPs also play an important role by virtue of their specific properties, since their presence, particularly in the virus seed, can decisively affect the population dynamics.

DIPs differ from standard virus particles (STVs) in that they posses one or more defective RNA segments. Due to this imperfection DIPs are unable to successfully accomplish their propagation as long as the respective host cell is not simultaneously coinfected by an STV. However, in the case of a coinfection with DIPs and STVs both virus subpopulations compete for resources, most notably for the RNA synthesis capacities of the host cell. As a result, the defective RNA segments are synthesized faster than their counterparts with standard configuration leading to the predominant release of DIPs 
[Nayak et al., 1989].

In order to model the effect of DIPs on a basic level additional types of infected cells have to be considered. Besides cells that have been infected by STVs (or active virus particles, respectively) there need to be DIP-infected as well as coinfected cells. This differentiation of the infected cells has also been applied by Frensing et al. [2013] in a lumped model of a continuous influenza vaccine production process, which is based on an earlier approach by Kirkwood and Bangham [1994]. In Fig. 42 a possible scheme is presented in which the principle influence of DIPs is considered within the framework of the established distributed population balance model of Müller et al. [2013] with additional regard to the differentiation between the eclipse phase and the latent phase. Note, that although the event of cell death is not graphically represented in Fig. 42, it must not be neglected in a respectively derived model.

With respect to a potential model formulation the general distinction according to the infecting virus particle results in two paths of progression. Cells that are infected by STVs in principle behave like the generic infected cells of the previously established model. The only and decisive deviation stems from the additional release of DIPs, which opens up the second path of possible progression. DIP-infected cells are assumed to stay passive until they are eventually coinfected by an STV and start to produce viral protein after an eclipse phase. In contrast to STV-infected cells coinfected cells are assumed to no longer release STVs, but a significantly higher percentage of DIPs instead. Furthermore, coinfected cells are assumed to become apoptotic and suffer the same consequences as STV-infected cells as a result, albeit a deviant behavior is thinkable.

When modeling the transition of STV-infected cells to the coinfected state the change of the release behavior may have to be considered in more detail than it is depicted in Fig. 42. Due to the time difference between a successful coinfection and the actual synthesis of defective viral RNA and the subsequent release of DIPs coinfected cells can be assumed to go through a transitional phase, during which the release of STVs is still possible (see Fig. 43). Depending on the time of coinfection and the duration of the subsequent latent phase the length and characteristics of this transitional phase may vary. For instance, if the coinfection happens after the STV-infected cell has passed its regular latent phase the release of STVs is not interrupted by the coinfection. Instead the release of STVs continues until the second latent phase is over, at which point the release of DIPs commences (Fig. 43a). If coinfection happens during the regular latent phase the respective cell, despite coinfection, starts to release STVs after the regular latent phase has been passed. As before, the subsequent release of DIPs begins with completion of the second latent phase (Fig. 43b).

Apparently, the presence of DIPs can have decisive influence on the dynamics of vaccine production processes and should therefore be considered in a future model formulation. 


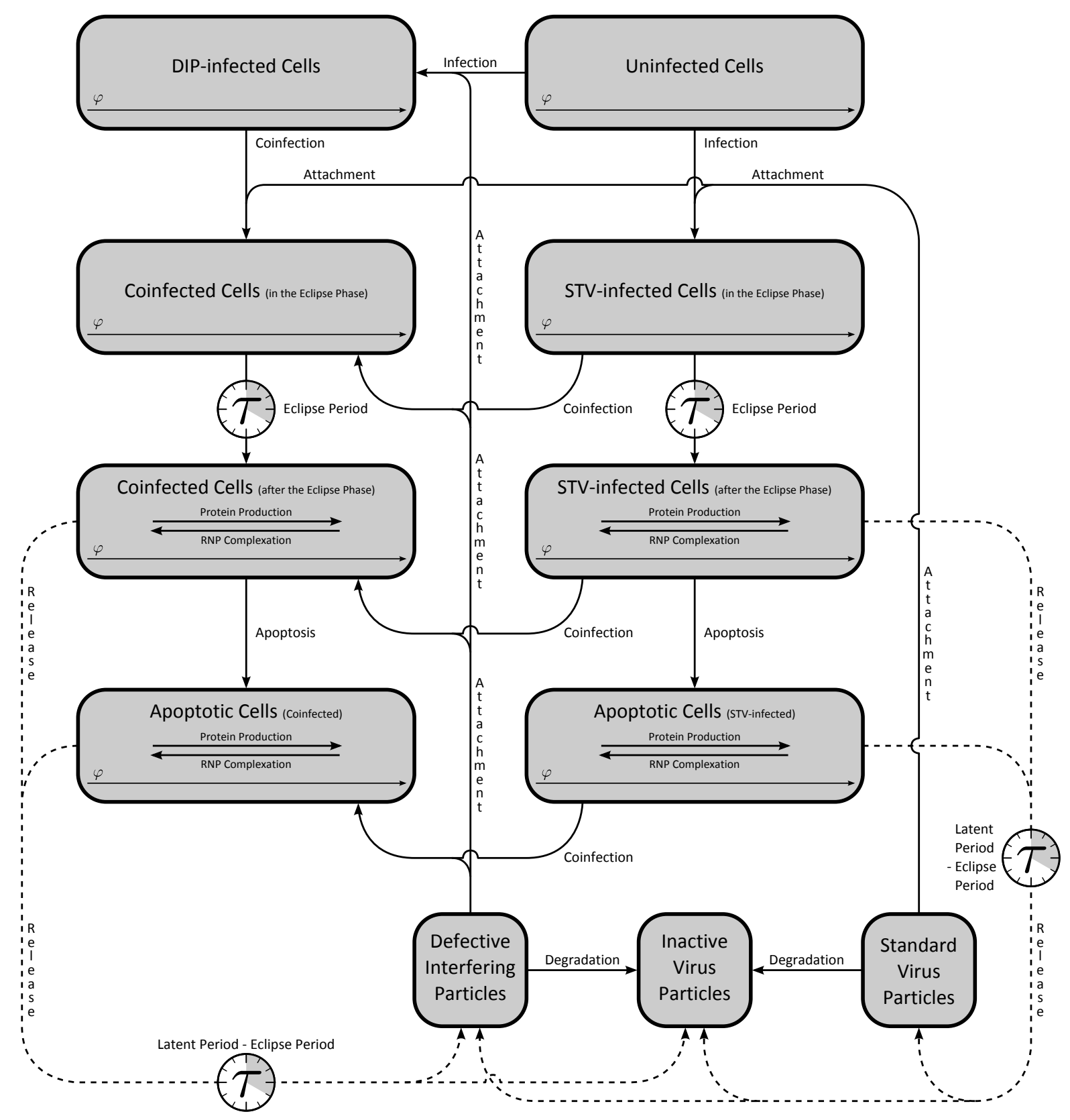

Figure 42: Possible scheme of a distributed model in consideration of the principal influence of defective interfering particles (DIPs). In addition, the differentiation between the eclipse phase and the latent phase is included. (Note that the event of cell death is not graphically represented.) Depending on the virus particle that successfully completes the initial infection, infected cells are divided into DIP-infected and STV-infected cells. The latter eventually release standard virus particles (STVs), inactive virus particles and a small fraction of DIPs, while the former are assumed to stay passive. After coinfection with a respective complementary virus particle the release of STVs ceases for the benefit of an increased fraction of released DIPs. 


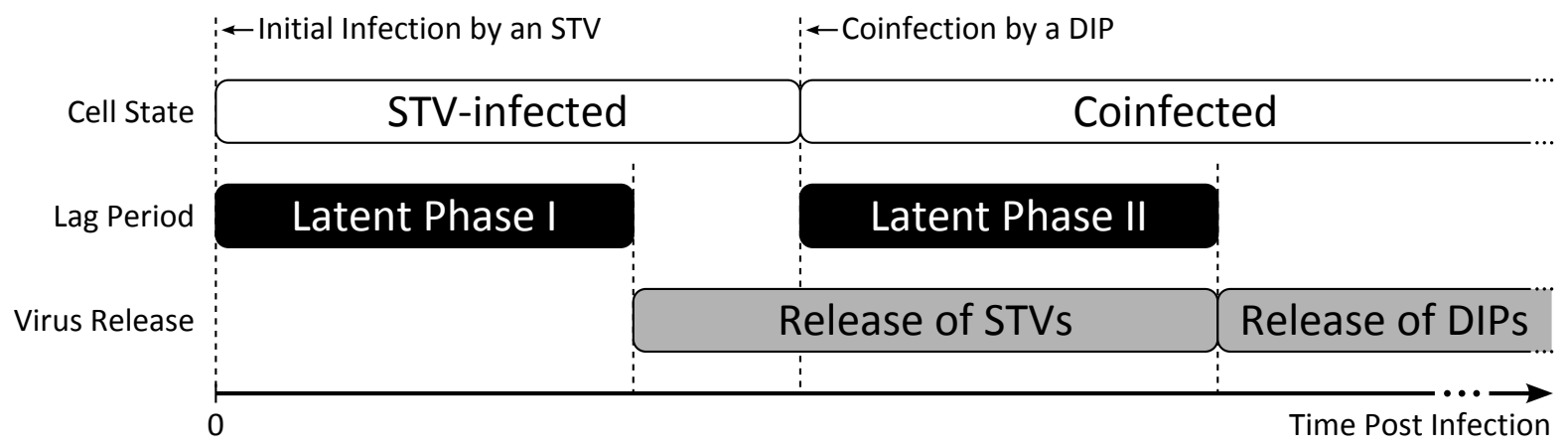

a) Coinfection after the latent phase of the STV-infected cell

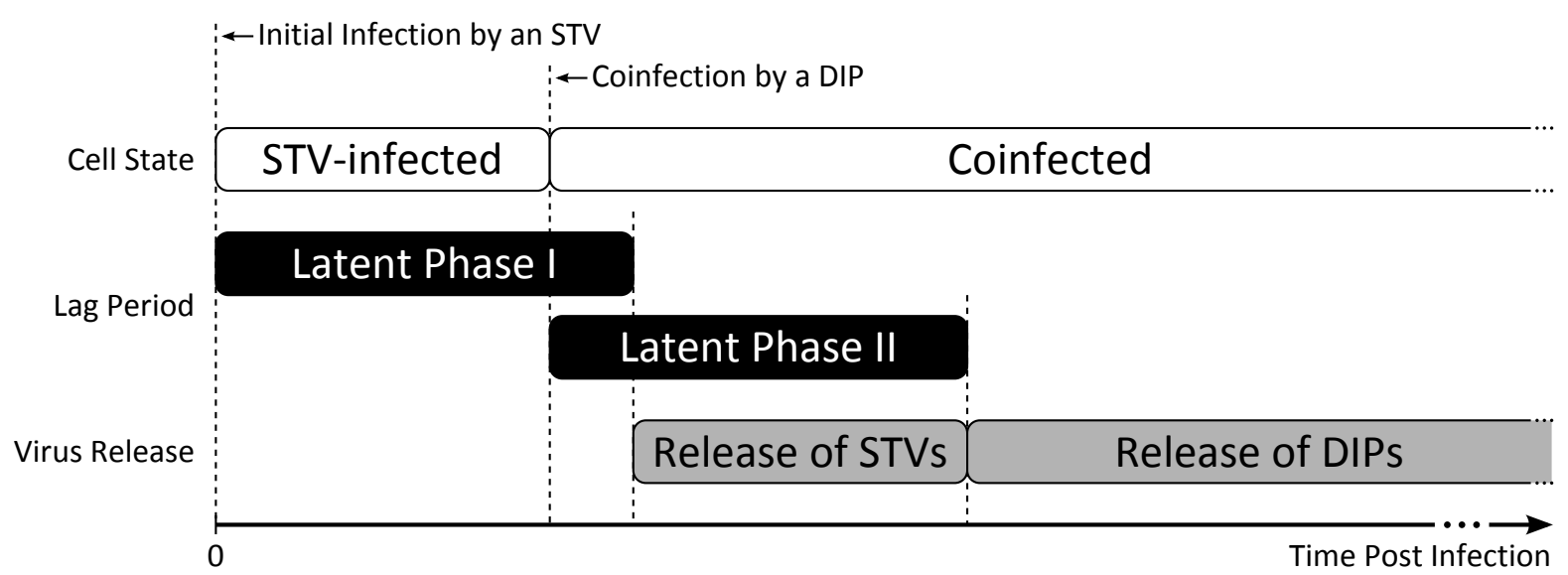

b) Coinfection during the latent phase of the STV-infected cell

Figure 43: The change of the virus release behavior during the transition of STV-infected cells to the coinfected state for two possible scenarios (STV: standard virus particle, DIP: defective interfering particle)

\subsubsection{Consideration of an immune response}

Depending on the applied virus strain the immune response of the host cell population can have a significant influence on the overall process dynamics, as the case of A/H1N1NIBSC indicates (see Section 5.8, page $78 \mathrm{ff}$.). In order to increase the convergence of experiment and simulation in a straight forward approach the elementary effects of the immune response have to be taken into account. The experimental distribution dynamics of A/H1N1-NIBSC (see Figs. 37-39) imply that host cells take up an antiviral state that either impedes successful infection or at least minimizes viral protein production. The promotion of the antiviral state depends on the secretion of cell signaling molecules (e.g. members of the type I interferon family) induced by viral infection [Ehrhardt et al., 2010; Heynisch et al., 2010].

Accordingly, virus induced cell signaling and the resulting antiviral state should be rep- 
resented in a future model formulation, for instance, by considering the production and release of signaling molecules that promote the transition into an antiviral state which significantly impedes viral replication. In Fig. 44 a possible model scheme is proposed.

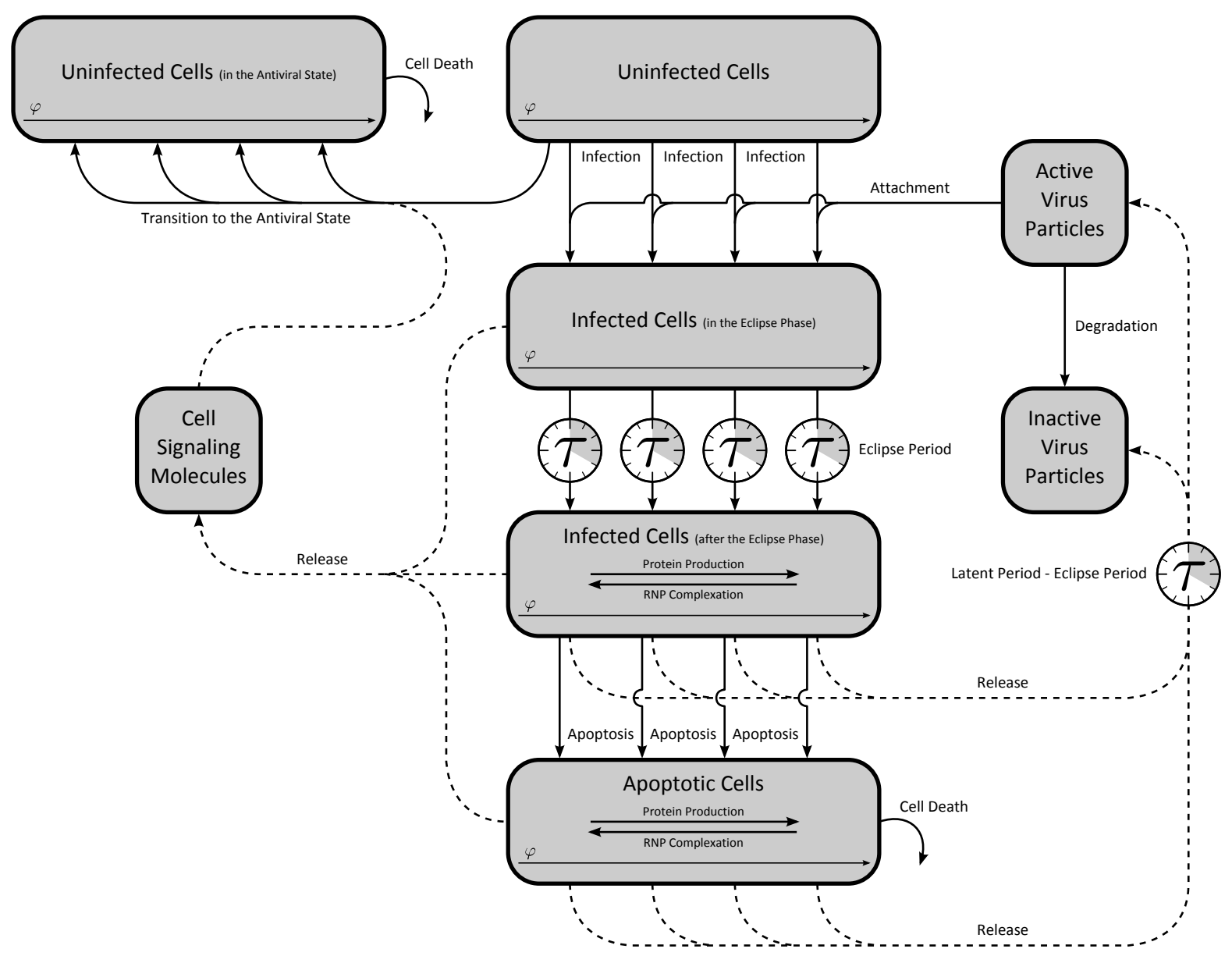

Figure 44: Scheme of a proposed model that considers the adoption of an antiviral state promoted by cell signaling. This scheme is based on an approach that includes the differentiation between the eclipse phase and the latent phase and adds the release of signaling molecules by infected and apoptotic cells. As a result, these signaling molecules promote the transition of uninfected cells to the antiviral state, which eventually inhibits successful infection or replication, respectively. 


\section{Appendix}

\section{A Determination of the limit of the scaling factor in Eq. (3.7)}

The limit of the scaling factor in Eq. (3.7) for $\Delta \varphi_{i} \rightarrow 0$ is determined with the help of L'Hospital's rule:

$$
\begin{aligned}
\lim _{\Delta \varphi_{i} \rightarrow 0} \frac{\lg \left(1+\Delta \varphi_{i} / \varphi_{i}\right)}{\Delta \varphi_{i}} & \stackrel{!}{=} \lim _{\Delta \varphi_{i} \rightarrow 0} \frac{\frac{\mathrm{d}}{\mathrm{d} \Delta \varphi_{i}} \lg \left(1+\Delta \varphi_{i} / \varphi_{i}\right)}{\frac{\mathrm{d}}{\mathrm{d} \Delta \varphi_{i}} \Delta \varphi_{i}} \\
& =\lim _{\Delta \varphi_{i} \rightarrow 0} \frac{\frac{\mathrm{d}}{\mathrm{d} \Delta \varphi_{i}} \frac{1}{\ln (10)} \ln \left(1+\Delta \varphi_{i} / \varphi_{i}\right)}{\frac{\mathrm{d}}{\mathrm{d} \Delta \varphi_{i}} \Delta \varphi_{i}} \\
& =\lim _{\Delta \varphi_{i} \rightarrow 0} \frac{\frac{1}{\ln (10)\left(1+\Delta \varphi_{i} / \varphi_{i}\right)} \frac{\mathrm{d}}{\mathrm{d} \Delta \varphi_{i}}\left(1+\Delta \varphi_{i} / \varphi_{i}\right)}{1} \\
& =\lim _{\Delta \varphi_{i} \rightarrow 0} \frac{\frac{1}{\ln (10)\left(1+\Delta \varphi_{i} / \varphi_{i}\right)} \frac{1}{\varphi_{i}}}{1} \\
& =\lim _{\Delta \varphi_{i} \rightarrow 0} \frac{1}{\ln (10)\left(\varphi_{i}+\Delta \varphi_{i}\right)} \\
& =\frac{1}{\ln (10) \varphi_{i}} .
\end{aligned}
$$




\section{B Derivation of Eq. (3.12)}

For the derivation of Eq. (3.12) the latent phase is represented by a transport system with the spatial coordinate $\lambda \in \mathbb{R},[0,1]$. The new state variable is marked with an additional hat symbol:

$$
\frac{\partial \hat{\tilde{L}}_{\mathrm{c}}(t, \varphi, \lambda)}{\partial t}=-\frac{1}{\tau} \frac{\partial \hat{\tilde{L}}_{\mathrm{c}}(t, \varphi, \lambda)}{\partial \lambda}-k_{\mathrm{cdv}} \hat{\tilde{L}}_{\mathrm{c}}(t, \varphi, \lambda) .
$$

Cells enter the transport system upon infection, which is specified by the boundary condition

$$
\hat{\tilde{L}}_{\mathrm{c}}(t, \varphi, \lambda=0)=\tau \cdot k_{\mathrm{vi}} \tilde{U}_{\mathrm{c}}(t, \varphi) V(t) .
$$

Eq. (3.12) is obtained by integration of Eq. (B.1) over the $\lambda$ domain:

$$
\begin{gathered}
\frac{\partial \tilde{L}_{\mathrm{c}}(t, \varphi)}{\partial t}=-\left.\frac{1}{\tau} \hat{\tilde{L}}_{\mathrm{c}}(t, \varphi, \lambda)\right|_{\lambda=0} ^{1}-k_{\mathrm{cdv}} \tilde{L}_{\mathrm{c}}(t, \varphi) \\
\text { with } \tilde{L}_{\mathrm{c}}(t, \varphi)=\int_{\lambda} \hat{\tilde{L}}_{\mathrm{c}}(t, \varphi, \lambda) \mathrm{d} \lambda .
\end{gathered}
$$

While the left boundary condition is given by Eq. (B.2), the number density at the right boundary of the transport system follows from the solution of Eq. (B.1). Therefore, a Laplace transformation is performed under consideration of the initial condition $\hat{\tilde{L}}_{\mathrm{c}}(t=$ $0, \varphi, \lambda)=0$ :

$$
\begin{aligned}
& s \hat{\tilde{L}}_{\mathrm{c}}^{*}(s, \varphi, \lambda)=-\frac{1}{\tau} \frac{\partial \hat{\tilde{L}}_{\mathrm{c}}^{*}(s, \varphi, \lambda)}{\partial \lambda}-k_{\mathrm{cdv}} \hat{\tilde{L}}_{\mathrm{c}}^{*}(s, \varphi, \lambda) . \\
& \text { with } \hat{\tilde{L}}_{\mathrm{c}}^{*}(s, \varphi, \lambda)=\mathscr{L}\left\{\hat{\tilde{L}}_{\mathrm{c}}\right\}
\end{aligned}
$$

After rearrangement Eq. (B.4) is integrated:

$$
\int_{\hat{\tilde{L}}_{\mathrm{c}}^{*}(s, \varphi, \lambda=0)}^{\hat{\tilde{L}}_{\mathrm{c}}^{*}(s, \varphi, \lambda)} \frac{\partial \hat{\tilde{L}}_{\mathrm{c}}^{*}(s, \varphi, \lambda)}{\hat{\tilde{L}}_{\mathrm{c}}^{*}(s, \varphi, \lambda)}=-\int_{\lambda=0}^{\lambda} \tau\left(s+k_{\mathrm{cdv}}\right) \partial \lambda
$$

yielding the solution in the complex variable domain:

$$
\begin{aligned}
\hat{\tilde{L}}_{\mathrm{c}}^{*}(s, \varphi, \lambda) & =\hat{\tilde{L}}_{\mathrm{c}}^{*}(s, \varphi, \lambda=0) \cdot \mathrm{e}^{-\tau\left(s+k_{\mathrm{cdv}}\right) \lambda} \\
& =\hat{\tilde{L}}_{\mathrm{c}}^{*}(s, \varphi, \lambda=0) \cdot \mathrm{e}^{-\tau s \lambda} \cdot \mathrm{e}^{-\tau k_{\mathrm{cdv}} \lambda} .
\end{aligned}
$$


Back transformation to the time domain and setting $\lambda=1$ yields the right boundary condition of Eq. (B.3):

$$
\begin{aligned}
\hat{\tilde{L}}_{\mathrm{c}}(t, \varphi, \lambda=1) & =\hat{\tilde{L}}_{\mathrm{c}}(t-\tau, \varphi, \lambda=0) \cdot \mathrm{e}^{-k_{\mathrm{cdv}} \tau} \\
& =\tau \cdot k_{\mathrm{vi}} \tilde{U}_{\mathrm{c}}(t-\tau, \varphi) V(t-\tau) \cdot \mathrm{e}^{-k_{\mathrm{cdv}} \tau}
\end{aligned}
$$

Finally, by combination of Eqs. (B.2), (B.3) and (B.6) the desired Eq. (3.12) is obtained:

$$
\frac{\partial \tilde{L}_{\mathrm{c}}(t, \varphi)}{\partial t}=k_{\mathrm{vi}} \tilde{U}_{\mathrm{c}}(t, \varphi) V(t)-k_{\mathrm{vi}} \tilde{U}_{\mathrm{c}}(t-\tau, \varphi) V(t-\tau) \cdot \mathrm{e}^{-k_{\mathrm{cdv}} \tau}-k_{\mathrm{cdv}} \tilde{L}_{\mathrm{c}}(t, \varphi)
$$




\section{Discretization via finite-volume method (FVM)}

\section{C.1 Discretization of Eq. (2.5)}

First, the transport coordinate $\lambda$ is discretized into $M$ equidistant control volumes of constant width

$$
\Delta \lambda=1 /(M-1) .
$$

In this way, upon reaching the last control volume, the transported cells have covered a distance of 1 . Hence, the transport coordinate $\lambda$ has to be defined in the interval from 0 to $1+\Delta \lambda$ (see Fig. 45). The left and right boundaries of control volume $i\left(\lambda_{i}\right.$ and $\left.\lambda_{i+1}\right)$ are determined by

$$
\lambda_{i}=\Delta \lambda \cdot(i-1) \quad \text { and } \quad \lambda_{i+1}=\Delta \lambda \cdot i \quad \text { with } \quad i \in \mathbb{N},[1, M] .
$$

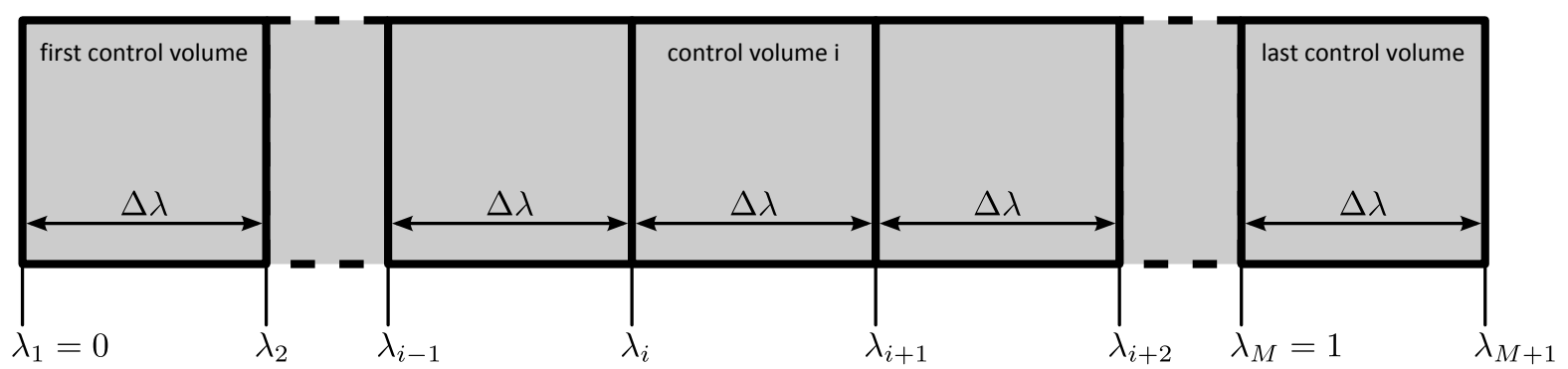

Figure 45: Equidistant discretization of the transport coordinate $\lambda \in[0,1+\Delta \lambda]$.

When assuming a constant course of $T_{\mathrm{c}}(t, \lambda)$ within a control volume, one can specify

$$
\left.\left.T_{\mathrm{c}}(t, \lambda)=T_{\mathrm{c}, i}(t) \quad \text { for } \quad\right] \lambda_{i}, \lambda_{i+1}\right] .
$$

The value of $T_{\mathrm{c}}$ at the left boundary of the first control volume is defined by the boundary condition (Eq. (2.6)):

$$
T_{\mathrm{c}}\left(t, \lambda_{1}\right)=T_{\mathrm{c}}(t, \lambda=0)=I_{\mathrm{c}}(t) .
$$

Now, Eq. (2.5) is discretized by integration within the limits of control volume $i$ :

$$
\begin{gathered}
\underbrace{\int_{\lambda_{i}}^{\lambda_{i+1}} \frac{\partial T_{\mathrm{c}}(t, \lambda)}{\partial t} \mathrm{~d} \lambda}_{\mathrm{A}}=-\underbrace{\frac{1}{\tau} \int_{\lambda_{i}}^{\lambda_{i+1}} \frac{\partial T_{\mathrm{c}}(t, \lambda)}{\partial \lambda} \mathrm{d} \lambda}_{\mathrm{B}}, \\
\mathrm{A}=\frac{\mathrm{d}}{\mathrm{d} t}\left[T_{\mathrm{c}} \lambda\right]_{\lambda_{i}}^{\lambda_{i+1}}=\frac{\mathrm{d} T_{\mathrm{c}, i}}{\mathrm{~d} t} \Delta \lambda,
\end{gathered}
$$




$$
\mathrm{B}=-\frac{1}{\tau}\left[T_{\mathrm{c}}\right]_{\lambda_{i}}^{\lambda_{i+1}}=-\frac{1}{\tau}\left(T_{\mathrm{c}, i}-T_{\mathrm{c}, i-1}\right) .
$$

In the case of term B an upwind scheme is applied. Concatenating terms A and B yields the discretized transport equation

$$
\begin{gathered}
\frac{\mathrm{d} T_{\mathrm{c}, i}(t)}{\mathrm{d} t}=-\frac{1}{\Delta \lambda \tau}\left(T_{\mathrm{c}, i}(t)-T_{\mathrm{c}, i-1}(t)\right) \\
\text { with } \quad T_{\mathrm{c}, 0}(t)=I_{\mathrm{c}}(t) .
\end{gathered}
$$

\section{Selection of the number of control volumes}

The number of control volumes $M$ affects the accuracy of the transport system. The higher the number of control volumes the sharper the front of transported cells, until all cells retain nearly the same retention time which equals $\tau$. A reduction of the number of control volumes results in a smearing effect and causes the retention time to become distributed around $\tau$.

Although hard delays are not desirable when modeling virus dynamics [Holder and Beauchemin, 2011], Möhler et al. decided to apply a hard delay. In the context of the work at hand this decision is adopted and a suitable number of control volumes is chosen. Therefore, simulations have been carried out with different numbers of control volumes:

$$
M_{j}=2^{j} \quad \text { for } \quad j \in \mathbb{N},[2,9] .
$$

Apart from that, the parameters published by Möhler et al. [2005] (see Tab. I, page 14) have been applied. The results are presented in Figure 46.

When modifying $M$ the time when $I_{\mathrm{c}}$ reaches its maximum $\left(t_{I_{\mathrm{c}, \max }}\right)$ changes. While the number of control volumes is doubled with each step the change of $t_{I_{\mathrm{c}, \max }}$ decreases (see Tab. V, page 103). In order to reach a suitable compromise between accuracy and computational effort the number of control volumes was chosen by looking for the step which results in a change of $t_{I_{\mathrm{c}, \max }}$ which is closest to one percent of the duration of the latent period $\tau$. It can be seen in Table $\mathrm{V}$, that a number of 64 control volumes complies with this requirement and is therefore chosen to be sufficiently accurate.

\section{C.2 Discretization of Eq. (3.18)}

Analog to Appendix C. $1 \lambda$ is discretized into $M=64$ equidistant control volumes of constant width $\Delta \lambda$ with $\lambda_{i}$ and $\lambda_{i+1}$ as the left and right boundary of control volume $i \in \mathbb{N},[1, M]$. 


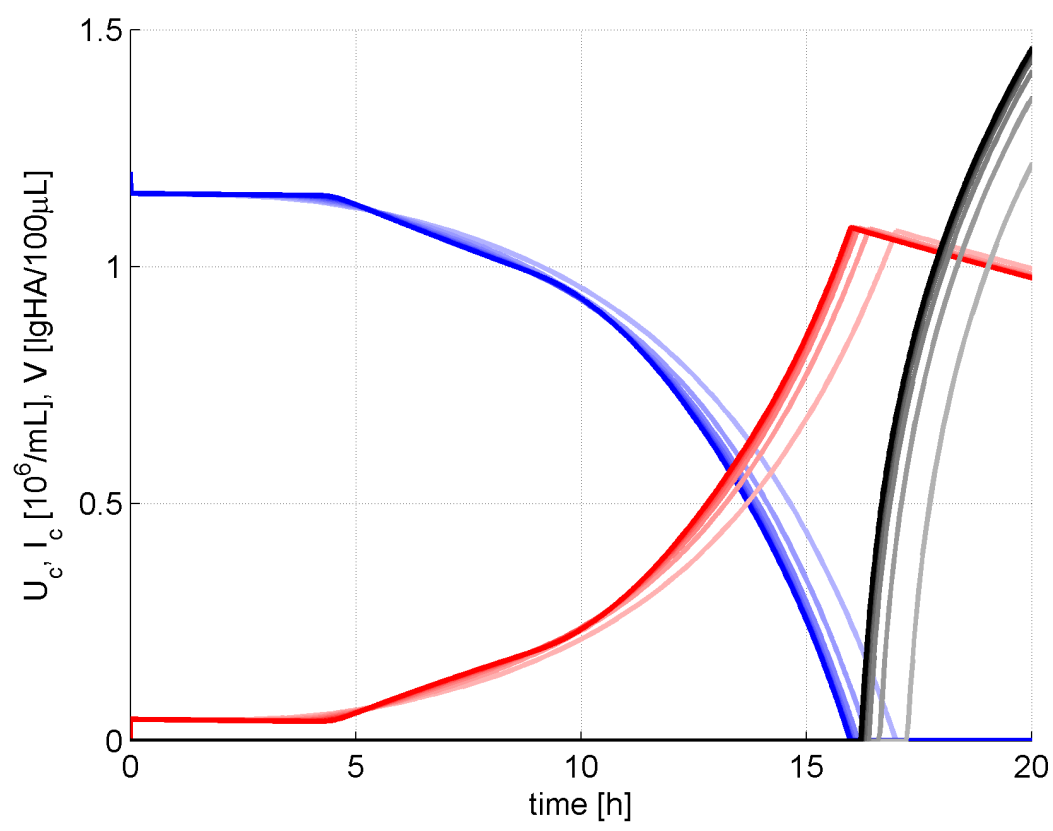

Figure 46: Simulation results for the model of Möhler et al. [2005] with the application of different numbers of control volumes $(M)$ for the discretized transport system. The concentrations of uninfected and infected cells are colored blue and red, respectively. The virus concentration is shown in gray. Results are drawn in 8 different shades of the respective color referring to 8 different numbers of control volumes. The lightest color represents $M=4$ while the most saturated color represents $M=512$. The number of control volumes is doubled from one step to the next. The rest of the parameters are taken from Möhler et al. [2005] (see Tab. I, page 14). Note, the individual waves of infection get more pronounced when the number of control volumes is increased.

\begin{tabular}{rccc}
\hline$M$ & $t_{I_{\mathrm{c}, \max }}[\mathrm{h}]$ & $\Delta t_{I_{\mathrm{c}, \max }}[\mathrm{h}]$ & $\Delta t_{I_{\mathrm{c}, \max }} / \tau[\%]$ \\
\hline 4 & 17.001 & - & - \\
8 & 16.398 & 0.603 & 13.394 \\
16 & 16.16 & 0.238 & 5.286 \\
32 & 16.06 & 0.1 & 2.216 \\
64 & 16.018 & 0.042 & 0.934 \\
128 & 16 & 0.018 & 0.403 \\
256 & 15.992 & 0.008 & 0.187 \\
512 & 15.988 & 0.004 & 0.09 \\
\hline
\end{tabular}

Table V: Selection of the number of control volumes of the discretized transport system. Simulations were carried out for different numbers of control volumes $(M)$. The table lists the time when $I_{\mathrm{c}}$ reaches its maximum $\left(t_{I_{\mathrm{c}, \max }}\right)$, the difference in $t_{I_{\mathrm{c}, \max }}$ from one simulation to the next $\left(\Delta t_{I_{\mathrm{c}, \max }}\right)$, and the ratio of $\Delta t_{I_{\mathrm{c}, \max }}$ to the duration of the latent period $\tau$. The step from 32 to 64 control volumes results in the change of $t_{I_{\mathrm{c}, \max }}$ which is closest to one percent of $\tau$. Hence, a number of 64 control volumes is chosen to be sufficiently accurate. 
Eq. (3.18) is discretized by integration within the limits of control volume $i$ :

$$
\begin{aligned}
\underbrace{\int_{\lambda_{i}}^{\lambda_{i+1}} \frac{\partial \hat{L}_{\mathrm{c}}(t, \lambda)}{\partial t} \mathrm{~d} \lambda}_{\mathrm{A}}=-\underbrace{\frac{1}{\tau} \int_{\lambda_{i}}^{\lambda_{i+1}} \frac{\partial \hat{L}_{\mathrm{c}}(t, \lambda)}{\partial \lambda} \mathrm{d} \lambda}_{\mathrm{B}}-\underbrace{k_{\mathrm{cdv}} \int_{\lambda_{i}}^{\lambda_{i+1}} \hat{L}_{\mathrm{c}}(t, \lambda) \mathrm{d} \lambda}_{\mathrm{C}}, \\
\mathrm{A}=\frac{\mathrm{d}}{\mathrm{d} t}\left[\hat{L}_{\mathrm{c}} \lambda\right]_{\lambda_{i}}^{\lambda_{i+1}}=\frac{\mathrm{d} \hat{L}_{\mathrm{c}, i}}{\mathrm{~d} t} \Delta \lambda, \\
\mathrm{B}=-\frac{1}{\tau}\left[\hat{L}_{\mathrm{c}}\right]_{\lambda_{i}}^{\lambda_{i+1}}=-\frac{1}{\tau}\left(\hat{L}_{\mathrm{c}, i}-\hat{L}_{\mathrm{c}, i-1}\right), \\
\mathrm{C}=-k_{\mathrm{cdv}}\left[\hat{L}_{\mathrm{c}} \lambda\right]_{\lambda_{i}}^{\lambda_{i+1}}=-k_{\mathrm{cdv}} \hat{L}_{\mathrm{c}, i} \Delta \lambda .
\end{aligned}
$$

In the case of term B an upwind scheme is applied. Summarizing terms A to C yields the discretized balance equation for cells in the latent phase:

$$
\begin{gathered}
\frac{\mathrm{d} \hat{L}_{\mathrm{c}, i}(t)}{\mathrm{d} t}=-\frac{1}{\Delta \lambda \tau}\left(\hat{L}_{\mathrm{c}, i}(t)-\hat{L}_{\mathrm{c}, i-1}(t)\right)-k_{\mathrm{cdv}} \hat{L}_{\mathrm{c}, i}(t) \\
\text { with } \quad \hat{L}_{\mathrm{c}, 0}(t)=\tau \cdot k_{\mathrm{vi}} U_{\mathrm{c}}(t) V(t) .
\end{gathered}
$$

\section{C.3 Discretization of Eq. (3.21)}

Similar to the sensor channels of the flow cytometer, the internal coordinate $\varphi$ is discretized into $N$ control volumes of logarithmically increasing width

$$
\begin{aligned}
& \Delta \varphi_{i}=\varphi_{i+1}-\varphi_{i}=\frac{1}{F_{\mathrm{VE}}}\left(10^{\frac{4}{N} \cdot i}-10^{\frac{4}{N} \cdot(i-1)}\right) \\
& \text { with } i \in \mathbb{N},[1, N] .
\end{aligned}
$$

In the equation above $\varphi_{i}$ and $\varphi_{i+1}$ are the left and right boundary of control volume $i$. Thus, the degree of fluorescence is confined within the intensity range of the flow cytometer $\left(1\right.$ to $\left.10^{4} \mathrm{FU}\right)$

$$
\varphi \in\left[\varphi_{\min }=1 \mathrm{FU} / F_{\mathrm{VE}}, \varphi_{\max }=10^{4} \mathrm{FU} / F_{\mathrm{VE}}\right]
$$

The course of $\tilde{I}_{\mathrm{c}}(t, \varphi)$ is assumed to be constant within a control volume, so that

$$
\begin{array}{r}
\left.\left.\tilde{I}_{\mathrm{c}}(t, \varphi)=\tilde{I}_{\mathrm{c}, i}(t) \quad \text { for }\right] \varphi_{i}, \varphi_{i+1}\right] \text { when an upwind scheme is applied, } \\
\text { or for }\left[\varphi_{i}, \varphi_{i+1}[\text { when a downwind scheme is applied. }\right.
\end{array}
$$


Eq. (3.21) is discretized by integrating within the limits of control volume $i$ :

$$
\begin{aligned}
& \underbrace{\int_{i+1}^{\varphi_{i+1}} \frac{\partial \tilde{I}_{\mathrm{c}}(t, \varphi)}{\partial t} \mathrm{~d} \varphi}_{\varphi_{i}}=\underbrace{\frac{\hat{L}_{\mathrm{c}, M}(t)}{\tau} \int_{\varphi_{i}}^{\varphi_{i+1}} q_{\mathrm{sim}, 0}(\varphi) \mathrm{d} \varphi}_{\mathrm{B}}-\underbrace{k_{\mathrm{pro}} \int_{\varphi_{i}}^{\varphi_{i+1}} \frac{\partial \tilde{I}_{\mathrm{c}}(t, \varphi)}{\partial \varphi} \mathrm{d} \varphi}_{\mathrm{C}} \\
& +\underbrace{k_{\mathrm{rel}} \int_{\varphi_{i}}^{\varphi_{i+1}} \frac{\partial \tilde{I}_{\mathrm{c}}(t, \varphi)}{\partial \varphi} \mathrm{d} \varphi}_{\mathrm{D}}-\underbrace{k_{\mathrm{cdv}} \int_{\varphi_{i}}^{\varphi_{i+1}} \tilde{I}_{\mathrm{c}}(t, \varphi) \mathrm{d} \varphi}_{\mathrm{E}}, \\
& \mathrm{A}=\frac{\mathrm{d}}{\mathrm{d} t}\left[\tilde{I}_{\mathrm{c}} \varphi\right]_{\varphi_{i}}^{\varphi_{i+1}}=\frac{\mathrm{d} \tilde{I}_{\mathrm{c}, i} \Delta \varphi_{i},}{\mathrm{~d} t} \\
& \mathrm{~B}=\frac{\hat{L}_{\mathrm{c}, M}\left[q_{\mathrm{sim}, 0}\right]_{\varphi_{i}}^{\varphi_{i+1}}=\frac{\hat{L}_{\mathrm{c}, M}}{\tau} q_{\mathrm{sim}, 0, i} \Delta \varphi_{i},}{\mathrm{C}=-k_{\mathrm{pro}}\left[\tilde{I}_{\mathrm{c}}\right]_{\varphi_{i}}^{\varphi_{i+1}}=-k_{\mathrm{pro}}\left(\tilde{I}_{\mathrm{c}, i}-\tilde{I}_{\mathrm{c}, i-1}\right),} \\
& \mathrm{D}=k_{\mathrm{rel}}\left[\tilde{I}_{\mathrm{c}}\right]_{\varphi_{i}}^{\varphi_{i+1}}=k_{\mathrm{rel}}\left(\tilde{I}_{\mathrm{c}, i+1}-\tilde{I}_{\mathrm{c}, i}\right), \\
& \mathrm{E}=-k_{\mathrm{cdv}}\left[\tilde{I}_{\mathrm{c}} \varphi\right]_{\varphi_{i}}^{\varphi_{i+1}}=-k_{\mathrm{cdv}} \tilde{I}_{\mathrm{c}, i} \Delta \varphi_{i} .
\end{aligned}
$$

While term $\mathrm{C}$ is conceived with an upwind scheme, for term $\mathrm{D}$ a downwind scheme is applied. Combining terms A to E yields the discretized balance equation for infected cells after the latent phase:

$$
\begin{aligned}
\frac{\mathrm{d} \tilde{I}_{\mathrm{c}, i}(t)}{\mathrm{d} t}= & \frac{\hat{L}_{\mathrm{c}, M}(t)}{\tau} q_{\mathrm{sim}, 0, i}-\frac{k_{\mathrm{pro}}}{\Delta \varphi_{i}}\left(\tilde{I}_{\mathrm{c}, i}(t)-\tilde{I}_{\mathrm{c}, i-1}(t)\right) \\
& +\frac{k_{\mathrm{rel}}}{\Delta \varphi_{i}}\left(\tilde{I}_{\mathrm{c}, i+1}(t)-\tilde{I}_{\mathrm{c}, i}(t)\right)-k_{\mathrm{cdv}} I_{\mathrm{c}, i}
\end{aligned}
$$

with $\quad \tilde{I}_{\mathrm{c}, 0}=\tilde{I}_{\mathrm{c}, N+1}=0$.

In addition, convection across the boundaries is prevented by defining $\tilde{I}_{\mathrm{c}, N}=0$ for the protein production term and $\tilde{I}_{\mathrm{c}, 1}=0$ for the virus release term. 


\section{C.4 Discretization of Eq. (5.10)}

Analog to Appendix C.3 the internal coordinate $\varphi$ is discretized into $N$ control volumes of logarithmically increasing width $\Delta \varphi_{i}$ with $\varphi_{i}$ and $\varphi_{i+1}$ as the left and right boundary of control volume $i \in \mathbb{N},[1, N]$.

The course of $\tilde{I}_{\mathrm{c}}(t, \varphi)$ is assumed to be constant within a control volume, so that

$$
\left.\left.\tilde{I}_{\mathrm{c}}(t, \varphi)=\tilde{I}_{\mathrm{c}, i}(t) \quad \text { for } \quad\right] \varphi_{i}, \varphi_{i+1}\right]
$$

The value of $\tilde{I}_{\mathrm{c}}$ at the left boundary of the first control volume is defined by the boundary condition:

$$
\tilde{I}_{\mathrm{c}}\left(t, \varphi_{1}\right)=\tilde{I}_{\mathrm{c}}(t, \varphi=0)=0 .
$$

Now, Eq. (5.10) is discretized by integrating within the limits of control volume $i$ :

$$
\begin{aligned}
& \underbrace{\int_{\varphi_{i}}^{\varphi_{i+1}} \frac{\partial \tilde{I}_{\mathrm{c}}(t, \varphi)}{\partial t} \mathrm{~d} \varphi}_{\mathrm{A}}=\underbrace{\frac{\hat{L}_{\mathrm{c}, M}(t)}{\tau} \int_{\varphi_{i}}^{\varphi_{i+1}} q_{\mathrm{sim}, 0}(\varphi) \mathrm{d} \varphi}_{\mathrm{B}}-\underbrace{\int_{\varphi_{i}}^{\varphi_{i+1}} \frac{\partial}{\partial \varphi}\left(k_{\mathrm{net}}(\varphi) \tilde{I}_{\mathrm{c}}(t, \varphi)\right) \mathrm{d} \varphi}_{\mathrm{C}} \\
& -\underbrace{\int_{\varphi_{i}+1}^{\varphi_{i}} k_{\mathrm{apo}}(\varphi) \tilde{I}_{\mathrm{c}}(t, \varphi) \mathrm{d} \varphi}_{\varphi_{i}}, \\
& \mathrm{~A}=\frac{\mathrm{d}}{\mathrm{d} t}\left[\tilde{I}_{\mathrm{c}} \varphi\right]_{\varphi_{i}}^{\varphi_{i+1}}=\frac{\mathrm{d} \tilde{I}_{\mathrm{c}, i} \Delta \varphi_{i},}{\mathrm{~d} t} \\
& \mathrm{~B}=\frac{\hat{L}_{\mathrm{c}, M}\left[q_{\mathrm{sim}, 0} \varphi\right]_{\varphi_{i}}^{\varphi_{i+1}}=\frac{\hat{L}_{\mathrm{c}, M}}{\tau} q_{\mathrm{sim}, 0, i} \Delta \varphi_{i},}{\mathrm{C}}=-\left[k_{\mathrm{net}} \tilde{I}_{\mathrm{c}}\right]_{\varphi_{i}}^{\varphi_{i+1}}=-\left(k_{\mathrm{net}, i} \tilde{I}_{\mathrm{c}, i}-k_{\mathrm{net}, i-1} \tilde{I}_{\mathrm{c}, i-1}\right), \\
& \mathrm{D}=-\left[k_{\mathrm{apo}} \tilde{I}_{\mathrm{c}} \varphi\right]_{\varphi_{i}}^{\varphi_{i+1}}=-k_{\mathrm{apo}, i} \tilde{I}_{\mathrm{c}, i} \Delta \varphi_{i} .
\end{aligned}
$$

An upwind scheme is applied to term C. Combining terms A to D yields the discretized balance equation:

$$
\begin{aligned}
& \frac{\mathrm{d} \tilde{I}_{\mathrm{c}, i}(t)}{\mathrm{d} t}=\frac{\hat{L}_{\mathrm{c}, M}(t)}{\tau} q_{\mathrm{sim}, 0, i}-\frac{1}{\Delta \varphi_{i}}\left(k_{\mathrm{net}, i} \tilde{I}_{\mathrm{c}, i}(t)-k_{\mathrm{net}, i-1} \tilde{I}_{\mathrm{c}, i-1}(t)\right)-k_{\mathrm{apo}, i} \tilde{I}_{\mathrm{c}, i}(t) \\
& \text { with } \quad \tilde{I}_{\mathrm{c}, 0}(t)=\tilde{I}_{\mathrm{c}, N}(t)=0 .
\end{aligned}
$$




\section{C.5 Discretization of Eq. (5.4)}

As shown in Appendix C.3 (page 104) $\varphi$ is discretized into $N$ control volumes of logarithmically increasing width $\Delta \varphi_{i}$ with $\varphi_{i}$ and $\varphi_{i+1}$ as the left and right boundary of control volume $i \in \mathbb{N},[1, N]$.

The course of $\tilde{A}_{\mathrm{c}}(t, \varphi)$ is assumed to be constant within a control volume, so that

$$
\tilde{A}_{\mathrm{c}}(t, \varphi)=\tilde{A}_{\mathrm{c}, i}(t) \quad \text { for } \quad\left[\varphi_{i}, \varphi_{i+1}[.\right.
$$

The value of $\tilde{A}_{\mathrm{c}}$ at the right boundary of the last control volume is defined by the boundary condition:

$$
\tilde{A}_{\mathrm{c}}\left(t, \varphi_{N+1}\right)=\tilde{A}_{\mathrm{c}}\left(t, \varphi=10^{4} \mathrm{FU} / F_{\mathrm{VE}}\right)=0 .
$$

Now, Eq. (5.4) is discretized by integrating within the limits of control volume $i$ :

$$
\begin{aligned}
& \underbrace{\varphi_{i+1} \frac{\partial \tilde{A}_{\mathrm{c}}(t, \varphi)}{\partial t} \mathrm{~d} \varphi}_{\varphi_{i}}= \underbrace{\int_{\varphi_{i}}^{\varphi_{i+1}} k_{\mathrm{apo}}(\varphi) \tilde{I}_{\mathrm{c}}(t, \varphi) \mathrm{d} \varphi}_{\mathrm{B}} \\
&-\underbrace{\int_{\varphi_{i}}^{\varphi_{i+1}} \frac{\partial}{\partial \varphi}\left(k_{\mathrm{net}, \mathrm{apo}}(\varphi) \tilde{A}_{\mathrm{c}}(t, \varphi)\right) \mathrm{d} \varphi}_{\mathrm{C}} \\
& \mathrm{A}=\frac{\mathrm{d}}{\mathrm{d} t}\left[\tilde{A}_{\mathrm{c}} \varphi\right]_{\varphi_{i}}^{\varphi_{i+1}}=-\underbrace{\int_{\varphi_{i}}^{\varphi_{i+1}} \tilde{A}_{\mathrm{cd}}(\varphi) \tilde{A}_{\mathrm{c}}(t, \varphi) \mathrm{d} \varphi}_{\mathrm{d} t}, \\
& \mathrm{~B}=\left[k_{\mathrm{apo}} \tilde{I}_{\mathrm{c}} \varphi\right]_{\varphi_{i}}^{\varphi_{i+1}}=k_{\mathrm{apo}, i} \tilde{I}_{\mathrm{c}, i} \Delta \varphi_{i}, \\
& \mathrm{C}=-\left[k_{\mathrm{net}, \mathrm{apo}} \tilde{A}_{\mathrm{c}}\right]_{\varphi_{i}}^{\varphi_{i+1}}=-\left(k_{\mathrm{net}, \mathrm{apo}, i+1} \tilde{A}_{\mathrm{c}, i+1}-k_{\mathrm{net}, \mathrm{apo}, i} \tilde{A}_{\mathrm{c}, i}\right), \\
& \mathrm{D}=-\left[k_{\mathrm{cd}} \tilde{A}_{\mathrm{c}} \varphi\right]_{\varphi_{i}}^{\varphi_{i+1}}=-k_{\mathrm{cd}, i} \tilde{A}_{\mathrm{c}, i} \Delta \varphi_{i} .
\end{aligned}
$$

A downwind scheme is applied to term C. Combining terms A to D yields the discretized 
balance equation:

$\frac{\mathrm{d} \tilde{A}_{\mathrm{c}, i}(t)}{\mathrm{d} t}=k_{\mathrm{apo}, i} \tilde{I}_{\mathrm{c}, i}(t)-\frac{1}{\Delta \varphi_{i}}\left(k_{\mathrm{net}, \mathrm{apo}, i+1} \tilde{A}_{\mathrm{c}, i+1}(t)-k_{\mathrm{net}, \mathrm{apo}, i} \tilde{A}_{\mathrm{c}, i}(t)\right)-k_{\mathrm{cd}, i} \tilde{A}_{\mathrm{c}, i}(t)$ with $\quad \tilde{A}_{\mathrm{c}, 1}(t)=\tilde{A}_{\mathrm{c}, N+1}(t)=0$. 


\section{Nomenclature}

\section{Abbreviations}

\begin{tabular}{|c|c|}
\hline A/Equi/H3N8 & Equine influenza A/Newmarket/1/93 \\
\hline $\mathrm{A} / \mathrm{H} 1 \mathrm{~N} 1$ & Human influenza A/Puerto Rico/8/34 \\
\hline $\mathrm{A} / \mathrm{H} 3 \mathrm{~N} 2$ & Reassortant of human influenza A/Wisconsin/67/2005 \\
\hline CAD & Caspase-activated deoxyribonuclease \\
\hline $\mathrm{CV}$ & Control volume \\
\hline DFF & DNA fragmentation factor \\
\hline $\mathrm{DIP}(\mathrm{s})$ & Defective interfering particle(s) \\
\hline DNA & Deoxyribonucleic acid \\
\hline dUTP & Deoxyuridine triphosphate \\
\hline FITC & Fluorescein isothiocyanate \\
\hline FU & Fluorescence units \\
\hline FVM & Finite-volume method \\
\hline $\mathrm{HA}$ & Hemagglutinin/Hemagglutination \\
\hline ICAD & Inhibitor of caspase-activated deoxyribonuclease \\
\hline IFN & Interferon \\
\hline M1 & Matrix protein 1 \\
\hline MDCK & Madin-Darby canine kidney \\
\hline MFI & Mean fluorescence intensity \\
\hline MOI & $\begin{array}{l}\text { Multiplicity of infection, number of infectious virus particles per un- } \\
\text { infected cell }\end{array}$ \\
\hline MOMP & Mitochondrial outer membrane permeabilization \\
\hline NA & Neuraminidase \\
\hline NIBSC & $\begin{array}{l}\text { National Institute for Biological Standards and Control (Hert- } \\
\text { fortshire) }\end{array}$ \\
\hline NP & Nucleoprotein \\
\hline NS1 & Nonstructural protein 1 \\
\hline PFU & Plaque forming unit \\
\hline p.i. & Post infection \\
\hline $\mathrm{RBC}$ & Red blood cell \\
\hline RKI & Robert Koch Institute (Berlin) \\
\hline RNA & Ribonucleic acid \\
\hline RNP & Ribonucleoprotein \\
\hline
\end{tabular}




$\begin{array}{ll}\text { STV }(\mathrm{s}) & \text { Standard virus particle(s) } \\ \text { TCID }_{50} & \text { Tissue culture infectious dose } \\ \text { TdT } & \text { Terminal deoxyribonucleotidyl transferase } \\ \text { TMR red } & \text { Tetramethylrhodamine } \\ \text { TNF } & \text { Tumor necrosis factor } \\ \text { TOI } & \text { Time of infection } \\ \text { TRAIL } & \text { TNF-related apoptosis-inducing ligand } \\ \text { TUNEL } & \text { TdT-mediated dUTP nick end labeling } \\ \text { VE } & \text { Virus equivalent }\end{array}$

\section{Latin letters}

$\begin{array}{lll}\tilde{A}_{\mathrm{c}}(t, \varphi) & {\left[\mathrm{ml}^{-1}\right]} & \text { Number density of apoptotic cells } \\ \tilde{A}_{\mathrm{c}, i}(t) & {\left[\mathrm{ml}^{-1}\right]} & \text { Number density of apoptotic cells in control volume } i \\ c_{i} & {\left[\mathrm{ml}^{-1}\right]} & \text { Cell concentration in control volume } i \\ C_{\mathrm{max}} & {\left[\mathrm{ml}^{-1}\right]} & \text { Maximum total number of cells } \\ c_{\mathrm{RBC}} & {\left[\mathrm{ml}^{-1}\right]} & \text { Concentration of red blood cells } \\ F & {[\mathrm{FU}]} & \text { Fluorescence intensity } \\ F_{\text {sensor }, i} & {[\mathrm{FU}]} & \text { Left boundary of sensor channel } i \\ F_{\text {sensor,m,i }} & {[\mathrm{FU}]} & \text { Mean fluorescence intensity of sensor channel } i \\ F_{\mathrm{VE}} & {[\mathrm{FU}]} & \text { Fluorescence intensity per virus equivalent } \\ I_{\mathrm{c}}(t) & {\left[\mathrm{ml}^{-1}\right]} & \text { Concentration of infected cells } \\ I_{\mathrm{c}, 0} & {\left[\mathrm{ml}^{-1}\right]} & \text { Initial concentration of infected cells } \\ \tilde{I}_{\mathrm{c}}(t, \varphi) & {\left[\mathrm{ml}^{-1}\right]} & \text { Number density of infected cells } \\ \tilde{I}_{\mathrm{c}, i}(t) & {\left[\mathrm{ml}^{-1}\right]} & \text { Number density of infected cells in control volume } i \\ \tilde{I}_{\mathrm{c}}^{\mathrm{I}}(t, \varphi) & {\left[\mathrm{ml}^{-1}\right]} & \text { Number density of infected cells in the first phase of viral } \\ \tilde{I}_{\mathrm{c}}^{\mathrm{II}}(t, \varphi) & {\left[\mathrm{ml}^{-1}\right]} & \text { replication } \\ & \text { Number density of infected cells in the second phase of viral } \\ k_{\mathrm{apo}} & {\left[\mathrm{h}^{-1}\right]} & \text { replication } \\ k_{\mathrm{bind}} & {\left[\mathrm{h}^{-1}\right]} & \text { Apoptosis coefficient } \\ k_{\mathrm{bind}, \text { apo }} & {\left[\mathrm{h}^{-1}\right]} & \text { NP binding coefficient } \\ k_{\mathrm{cd}} & {\left[\mathrm{h}^{-1}\right]} & \text { NP binding coefficient of apoptotic cells } \\ k_{\mathrm{cdf}} & {\left[\mathrm{h}^{-1}\right]} & \text { Cell death coefficient } \\ k_{\mathrm{cdv}} & {\left[\mathrm{h}^{-1}\right]} & \text { Cell death coefficient before viral infection } \\ k_{\mathrm{deg}} & {\left[\mathrm{h}^{-1}\right]} & \text { Cell death coefficient after viral infection } \\ & & \text { Virus degradation coefficient }\end{array}$




\begin{tabular}{|c|c|c|}
\hline$k_{\text {net }}$ & {$\left[\mathrm{h}^{-1}\right]$} & Net coefficient \\
\hline$k_{\text {net,apo }}$ & {$\left[\mathrm{h}^{-1}\right]$} & Net coefficient of apoptotic cells \\
\hline$k_{\text {pro }}$ & {$\left[\mathrm{h}^{-1}\right]$} & Protein production coefficient \\
\hline$k_{\text {pro,apo }}$ & {$\left[\mathrm{h}^{-1}\right]$} & Protein production coefficient of apoptotic cells \\
\hline$k_{\text {pro }}^{\mathrm{I}}$ & {$\left[\mathrm{h}^{-1}\right]$} & $\begin{array}{l}\text { Protein production coefficient in the first phase of viral } \\
\text { replication }\end{array}$ \\
\hline$k_{\text {pro }}^{\mathrm{II}}$ & {$\left[\mathrm{h}^{-1}\right]$} & $\begin{array}{l}\text { Protein production coefficient in the second phase of viral } \\
\text { replication }\end{array}$ \\
\hline$k_{\mathrm{pt}}$ & {$\left[\mathrm{h}^{-1}\right]$} & Phase transition coefficient \\
\hline$k_{\mathrm{pt}, \max }$ & {$\left[\mathrm{h}^{-1}\right]$} & Maximum phase transition coefficient \\
\hline$k_{\mathrm{pt}, \min }$ & {$\left[\mathrm{h}^{-1}\right]$} & Minimum phase transition coefficient \\
\hline$k_{\text {rel }}$ & {$\left[\mathrm{h}^{-1}\right]$} & Virus release coefficient \\
\hline$k_{\mathrm{va}}$ & {$[\mathrm{ml} / \mathrm{h}]$} & Virus attachment coefficient \\
\hline$k_{\mathrm{vd}}$ & {$\left[\mathrm{h}^{-1}\right]$} & Virus degradation coefficient \\
\hline$k_{\mathrm{vi}}$ & {$[\mathrm{ml} / \mathrm{h}]$} & Infection coefficient \\
\hline$L_{\mathrm{c}}(t)$ & {$\left[\mathrm{ml}^{-1}\right]$} & Concentration of infected cells during the latent phase \\
\hline$\tilde{L}_{\mathrm{c}}(t, \varphi)$ & {$\left[\mathrm{ml}^{-1}\right]$} & $\begin{array}{l}\text { Number density (with respect to } \varphi \text { ) of infected cells during } \\
\text { the latent phase }\end{array}$ \\
\hline$\hat{L}_{\mathrm{c}}(t, \lambda)$ & {$\left[\mathrm{ml}^{-1}\right]$} & $\begin{array}{l}\text { Number density (with respect to } \lambda \text { ) of infected cells during } \\
\text { the latent phase }\end{array}$ \\
\hline$\hat{L}_{\mathrm{c}, i}(t)$ & {$\left[\mathrm{ml}^{-1}\right]$} & $\begin{array}{l}\text { Number density of infected cells during the latent phase in } \\
\text { control volume } i\end{array}$ \\
\hline$\tilde{\tilde{L}}_{\mathrm{c}}(t, \varphi, \lambda)$ & {$\left[\mathrm{ml}^{-1}\right]$} & Number density of infected cells during the latent phase \\
\hline$\hat{\tilde{L}}_{\mathrm{c}}^{*}(s, \varphi, \lambda)$ & {$\left[\mathrm{ml}^{-1}\right]$} & Laplace transform of $\hat{\tilde{L}}_{\mathrm{c}}(t, \varphi, \lambda)$ \\
\hline$N$ & {$[-]$} & Number \\
\hline$N_{\mathrm{CV}}$ & {$[-]$} & Number of control volumes \\
\hline$N_{\text {sensor }}$ & {$[-]$} & Number sensor channel of flow cytometer \\
\hline M & {$[-]$} & Number \\
\hline$P_{\text {eff }}$ & {$[-]$} & $\begin{array}{l}\text { Plating efficiency, number of infectious virions per total } \\
\text { number of virus particles }\end{array}$ \\
\hline$q_{\text {approx }, i, 0}$ & {$[1 /$ "lg FU"] } & $\begin{array}{l}\text { Approximated initial cell distribution of the experiment for } \\
\text { sensor channel } i\end{array}$ \\
\hline$q_{\mathrm{c}, i}$ & [1/"lg FU"] & $\begin{array}{l}\text { Normalized cell distribution of the simulation in control vol- } \\
\text { ume } i\end{array}$ \\
\hline$q_{\mathrm{exp}, i}$ & [1/"lg FU"] & Experimental cell distribution for sensor channel $i$ \\
\hline$q_{\mathrm{sim}, 0}(\varphi)$ & {$[-]$} & Normalized initial cell distribution of the simulation \\
\hline$q_{\mathrm{sim}, i, 0}$ & {$[-]$} & $\begin{array}{l}\text { Normalized initial cell distribution of the simulation in con- } \\
\text { trol volume } i\end{array}$ \\
\hline
\end{tabular}




\begin{tabular}{|c|c|c|}
\hline$t$ & {$[\mathrm{~h}]$} & Time \\
\hline$T_{\mathrm{c}}(t, \lambda)$ & {$\left[\mathrm{ml}^{-1}\right]$} & Generic number density within the transport system \\
\hline$t_{\text {shift }}$ & {$[\mathrm{h}]$} & $\begin{array}{l}\text { Amount of time that simulation results are shifted to ap- } \\
\text { proximate the lag period }\end{array}$ \\
\hline$U_{\mathrm{c}}(t)$ & {$\left[\mathrm{ml}^{-1}\right]$} & Concentration of uninfected cells \\
\hline$U_{\mathrm{c}, 0}$ & {$\left[\mathrm{ml}^{-1}\right]$} & Initial concentration of uninfected cells \\
\hline$\tilde{U}_{\mathrm{c}}(t, \varphi)$ & {$\left[\mathrm{ml}^{-1}\right]$} & Number density of uninfected cells \\
\hline$V$ & {$\left[\mathrm{ml}^{-1}\right]$} & Total virus concentration \\
\hline$V_{0}$ & {$\left[\mathrm{ml}^{-1}\right]$} & Initial total virus concentration \\
\hline$V_{\text {ac }}$ & {$\left[\mathrm{ml}^{-1}\right]$} & Concentration of active/infectious virus particles \\
\hline$V_{\mathrm{ac}, 0}$ & {$\left[\mathrm{ml}^{-1}\right]$} & Initial concentration of active/infectious virus particles \\
\hline$V_{\text {inac }}$ & {$\left[\mathrm{ml}^{-1}\right]$} & Concentration of inactive/uninfectious virus particles \\
\hline$V_{\text {inac }, 0}$ & {$\left[\mathrm{ml}^{-1}\right]$} & Initial concentration of inactive/uninfectious virus particles \\
\hline$V_{\text {inf,seed }}$ & {$\left[\mathrm{ml}^{-1}\right]$} & Concentration of infectious virus particles in the virus seed \\
\hline$V_{\text {seed }}$ & {$\left[\mathrm{ml}^{-1}\right]$} & Total virus concentration of the virus seed \\
\hline$Z_{i}$ & {$[-]$} & Total number of cells assigned to sensor channel $i$ \\
\hline
\end{tabular}

\section{Greek letters}

$\begin{array}{lll}\alpha & {[-]} & \text { Generic internal coordinate } \\ \delta & {[-]} & \text { Degree of infection } \\ \Delta F_{\mathrm{lg}} & {[\text { "lg FU"] }} & \text { Logarithmic width of a control volume } \\ \Delta F_{\text {sensor }, i} & {[\mathrm{FU}]} & \text { Width of sensor channel } i \\ \Delta F_{\text {sensor,lg }} & {[\text { "lg FU"] }} & \text { Logarithmic width of a sensor channel } \\ \Delta \lambda & {[-]} & \text { Width of one control volume of the discretized transport sys- } \\ \Delta \varphi_{i} & {[-]} & \text { tem } \\ \lambda & {[-]} & \text { Width of control volume } i \text { of the degree of fluorescence } \\ \mu_{0} & {[\mathrm{FU}]} & \text { Expectation of the initial cell distribution } \\ \mu_{\mathrm{c}} & {\left[\mathrm{h}^{-1}\right]} & \text { Cell growth coefficient } \\ \mu_{\mathrm{c}, \text { max }} & {\left[\mathrm{h}^{-1}\right]} & \text { Maximum cell growth coefficient } \\ \mu_{\mathrm{pt}} & {[\mathrm{FU}]} & \text { Expectation of the phase transition coefficient } \\ \sigma_{0} & {[\mathrm{FU}]} & \text { Standard deviation of the initial cell distribution } \\ \sigma_{\mathrm{pt}} & {[\mathrm{FU}]} & \text { Standard deviation of the phase transition coefficient } \\ \tau & {[\mathrm{h}]} & \text { Length of the latent period } \\ \varphi & {[-]} & \text { Degree of fluorescence }\end{array}$




$\begin{array}{lll}\varphi_{i} & {[-]} & \begin{array}{l}\text { Left boundary of the degree of fluorescence of control volume } \\ i\end{array} \\ \varphi_{\mathrm{m}, i} & {[-]} & \text { Mean degree of fluorescence of control volume } i \\ \varphi_{\max } & {[-]} & \text { Maximum degree of fluorescence } \\ \varphi_{\min } & {[-]} & \text { Minimum degree of fluorescence }\end{array}$




\section{List of Tables}

I Parameter set and initial conditions for the model of Möhler et al. [2005] 14

II Parameter sets and initial conditions for the extended model of Müller et al. [2008] . . . . . . . . . . . . . . . . . . . . . . . . . . . . . 28

III Parameter set and initial conditions for the "two-phase model" at MOI =

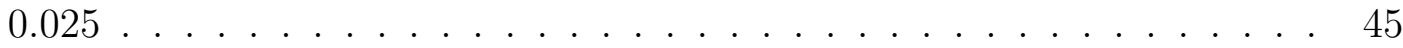

IV Parameter sets and initial conditions for the model of Müller et al. [2013] 64

$\mathrm{V}$ Selection of the number of control volumes of the discretized transport system . . . . . . . . . . . . . . . . . . . 103 


\section{List of Figures}

1 Example of flow cytometric data that reveal characteristic dynamic phenomena of the cell population . . . . . . . . . . . . . . . . .

2 Illustration of a bioreactor with a magnified detail depicting confluently overgrown microcarriers. . . . . . . . . . . . . . . . 5 5

3 Scheme of the model of Möhler et al. [2005] . . . . . . . . . . . . . 11

4 Scheme of the model of Möhler et al. [2005] with the latent period being approximated by a discretized transport system . . . . . . . . . . . . . 12

5 Influence of the attachment coefficient $\left(k_{\mathrm{va}}\right)$ and the infection coefficient $\left(k_{\mathrm{vi}}\right)$ on the dynamics of the model of Möhler et al. [2005] . . . . . . . . . 13

6 Simulation results of the model of Möhler et al. [2005] . . . . . . . . . . 16

7 Results of flow cytometric measurements conducted by Schulze-Horsel et al. [2008] for the replication of equine influenza A . . . . . . . . . . . . 18

8 Raw flow cytometric data vs. experimental cell distribution . . . . . . . . 20

$9 \quad$ Initial cell distributions and their approximation . . . . . . . . . . . . . 21

10 Scheme of the extended model of Müller et al. [2008] . . . . . . . . . . . 24

11 Scheme of the discretized, extended model of Müller et al. [2008] . . . . . 27

12 Integral dynamics of the extended model of Müller et al. [2008] with $k_{\mathrm{vi}}=$ $1.9 \cdot 10^{-3} \mathrm{ml} / \mathrm{h} \ldots \ldots \ldots \ldots \ldots$

13 Comparison of the integral dynamics of the extended model of Müller et al. [2008] with experimental data . . . . . . . . . . . . . . . . . . 33

14 Comparison of the mean fluorescence intensities of the extended model of Müller et al. [2008] with experimental data . . . . . . . . . . . . . . . . . 35

15 Comparison of the distribution dynamics of the extended model of Müller et al. [2008] with experimental data at MOI $=0.025$ (Part I) . . . . . . 36

16 Comparison of the distribution dynamics of the extended model of Müller et al. [2008] with experimental data at MOI $=0.025$ (Part II) . . . . . . 37

17 Comparison of the distribution dynamics of the extended model of Müller et al. [2008] with experimental data at MOI $=1.0$ (Part I) $\ldots . . . \quad . \quad 38$

18 Comparison of the distribution dynamics of the extended model of Müller et al. [2008] with experimental data at MOI $=1.0$ (Part II) . . . . . . . . .

19 Comparison of the distribution dynamics of the extended model of Müller et al. [2008] with experimental data at MOI $=3.0$ (Part I) . . . . . . . .

20 Comparison of the distribution dynamics of the extended model of Müller et al. [2008] with experimental data at MOI $=3.0$ (Part II) . . . . . . . . .

21 Comparison of the mean fluorescence intensity of the "two-phase model" with experimental data . . . . . . . . . . . . . . . . .

22 Comparison of the distribution dynamics of the "two-phase model" with experimental data (Part I) . . . . . . . . . . . . . . . .

23 Comparison of the distribution dynamics of the "two-phase model" with experimental data $($ Part II $\ldots \ldots \ldots \ldots$

(1)

3

6

8

0

4

27


24 Main phases of the virus replication process . . . . . . . . . . . 55

25 Scheme of the model of Müller et al. [2013] . . . . . . . . . . . . . . 57

26 Scheme of the discretized model of Müller et al. [2013] . . . . . . . . . . 60

27 Comparison of the integral dynamics of the model of Müller et al. [2013] with experimental data . . . . . . . . . . . . . . . . 65

28 Comparison of the mean fluorescence intensities of the model of Müller et al. [2013] with experimental data . . . . . . . . . . . . . . . . 68

29 Comparison of the distribution dynamics of the model of Müller et al. [2013] with experimental data for A/H1N1-RKI (Part I) . . . . . . . . . 71

30 Comparison of the distribution dynamics of the model of Müller et al. [2013] with experimental data for A/H1N1-RKI (Part II) . . . . . . . . . 72

31 Comparison of the distribution dynamics of the model of Müller et al. [2013] with experimental data for A/H1N1-RKI (Part III) . . . . . . . . 73

32 Comparison of the distribution dynamics of the model of Müller et al. [2013] with experimental data for A/H3N2, Experiment 1 (Part I) . . . . 74

33 Comparison of the distribution dynamics of the model of Müller et al. [2013] with experimental data for A/H3N2, Experiment 1 (Part II) . . 75

34 Comparison of the distribution dynamics of the model of Müller et al. [2013] with experimental data for A/H3N2, Experiment 2 (Part I) . . . . 76

35 Comparison of the distribution dynamics of the model of Müller et al. [2013] with experimental data for A/H3N2, Experiment 2 (Part II) . . 77

36 Experimental distribution dynamics for A/H1N1-NIBSC, Experiment 1 (Part I) . . . . . . . . . . . . . . . . . . . . . . . . . . . . 79

37 Experimental distribution dynamics for A/H1N1-NIBSC, Experiment 1 (Part II) . . . . . . . . . . . . . . . . . . . . . . . . . . . . . . 80

38 Experimental distribution dynamics for A/H1N1-NIBSC, Experiment 2 (Part I) [Schulze-Horsel et al., 2009]. . . . . . . . . . . . . . . . . . . . 82

39 Experimental distribution dynamics for A/H1N1-NIBSC, Experiment 2 (Part II) [Schulze-Horsel et al., 2009] . . . . . . . . . . . . . . . . . 83

40 Example of two-dimensional flow cytometric data . . . . . . . . . . . . 88

41 Scheme of a proposed model in consideration of the distinction between the eclipse phase and the latent phase . . . . . . . . . . . . . . . . . . 91

42 Possible scheme of a distributed model in consideration of the principal influence of defective interfering particles . . . . . . . . . . . . . . . 94

43 The change of the virus release behavior during the transition of STVinfected cells to the coinfected state . . . . . . . . . . . . . 95

44 Scheme of a proposed model that considers the adoption of an antiviral state promoted by cell signaling . . . . . . . . . . . . . . . 96

45 Equidistant discretization of the transport coordinate $\lambda \in[0,1+\Delta \lambda]$. . . 101

46 Simulation results for the model of Möhler et al. [2005] with the application of different numbers of control volumes for the discretized transport system . . . . . . . . . . . . . . . . . . . 103 


\section{References}

Albeck, J. G., Burke, J. M., Spencer, S. L., Lauffenburger, D. A., Sorger, P. K., 2008. Modeling a snap-action, variable-delay switch controlling extrinsic cell death. PLoS Biology 6 (12), e299.

Alberts, B., Johnson, A., Lewis, J., Raff, M., Roberts, K., Walter, P., 2008. Molecular Biology of The Cell, 5th Edition. Garland Science, New York.

Arranz, R., Coloma, R., Chichón, F. J., Conesa, J. J., Carrascosa, J. L., Valpuesta, J. M., Ortín, J., Martín-Benito, J., 2012. The structure of native influenza virion ribonucleoproteins. Science 338 (6114), 1634-1637.

Banks, H. T., Sutton, K. L., Thompson, W. C., Bocharov, G., Roose, D., Schenkel, T., Meyerhans, A., Jan. 2011. Estimation of cell proliferation dynamics using CFSE data. Bulletin of Mathematical Biology 73 (1), 116-150.

Belshe, R. B., Sep. 2010. The need for quadrivalent vaccine against seasonal influenza. Vaccine 28, D45-D53, Supplement 4.

Bialik, S., Zalckvar, E., Ber, Y., Rubinstein, A. D., Kimchi, A., 2010. Systems biology analysis of programmed cell death. Trends in Biochemical Sciences 35 (10), 556-564.

Bollum, F. J., 1978. Terminal deoxynucleotidyl transferase: biological studies. In: Meister, A. (Ed.), Advances in Enzymology and Related Areas of Molecular Biology. Vol. 47. John Wiley \& Sons, New York, pp. 347-374.

Brydon, E. W. A., Morris, S. J., Sweet, C., 2005. Role of apoptosis and cytokines in influenza virus morbidity. FEMS Microbiology Reviews 29 (4), 837-850.

Burleson, F. G., Chambers, T. M., Wiedbrauk, D. L., 1992. Virology: a laboratory manual. Academic press, London.

Caracotsios, M., Stewart, W. E., 1985. Sensitivity analysis of initial value problems with mixed ODEs and algebraic equations. Computers \& Chemical Engineering 9 (4), 359-365.

de Gooijer, C. D., Koken, R. H. M., van Lier, F. L. J., Kool, M., Vlak, J. M., Tramper, J., Aug. 1992. A structured dynamic model for the baculovirus infection process in insect-cell reactor configurations. Biotechnology and Bioengineering 40 (4), 537-548.

Domachowske, J. B., Pankow-Culot, H., Bautista, M., Feng, Y., Claeys, C., Peeters, 
M., Innis, B. L., Jain, V., Jun. 2013. A randomized trial of candidate inactivated quadrivalent influenza vaccine versus trivalent influenza vaccines in children aged 317 years. Journal of Infectious Diseases 207 (12), 1878-1887.

Dürr, R., Müller, T., Isken, B., Schulze-Horsel, J., Reichl, U., Kienle, A., 2012. Distributed modeling and parameter estimation of influenza virus replication during vaccine production. In: Troch, I., Breitenecker, F. (Eds.), $7^{\text {th }}$ Vienna International Conference on Mathematical Modelling. pp. 320-325.

Earnshaw, W. C., 1995. Nuclear changes in apoptosis. Current Opinion in Cell Biology $7(3), 337-343$.

Ehrhardt, C., Seyer, R., Hrincius, E. R., Eierhoff, T., Wolff, T., Ludwig, S., 2010. Interplay between influenza A virus and the innate immune signaling. Microbes and Infection 12 (1), 81-87.

Ehrhardt, C., Wolff, T., Pleschka, S., Planz, O., Beermann, W., Bode, J. G., Schmolke, M., Ludwig, S., 2007. Influenza A virus NS1 protein activates the PI3K/Akt pathway to mediate antiapoptotic signaling responses. Journal of Virology 81 (7), 3058-3067.

Enari, M., Sakahira, H., Yokoyama, H., Okawa, K., Iwamatsu, A., Nagata, S., 1998. A caspase-activated DNase that degrades DNA during apoptosis, and its inhibitor ICAD. Nature 391 (6662), 43-50.

Enden, G., Zhang, Y. H., Merchuk, J. C., Dec. 2005. A model of the dynamics of insect cell infection at low multiplicity of infection. Journal of Theoretical Biology 237 (3), $257-264$.

Englezos, P., Kalogerakis, N., 2001. Applied Parameter Estimation for Chemical Engineers. Marcel Dekker, Inc.

Flint, S. J., Enquist, L. W., Racaniello, V. R., Skalka, A. M., 2009. Principles of Virology, 3rd Edition. Vol. 1 - Molecular Biology. ASM Press, Washington, DC.

Fredrickson, A., 1991. Segregated, structured, distributed models and their role in microbial ecology: A case study based on work done on the filter-feeding ciliate Tetrahymena pyriformis. Microbial Ecology 22 (1), 139-159.

Fredrickson, A., 2003. Population balance equations for cell and microbial cultures revisited. AIChE Journal 49 (4), 1050-1059. 
Fredrickson, A., Mantzaris, N., Jun. 2002. A new set of population balance equations for microbial and cell cultures. Chemical Engineering Science 57 (12), 2265-2278.

Fredrickson, A., Ramkrishna, D., Tsuchiya, H. M., Sep. 1967. Statistics and dynamics of procaryotic cell populations. Mathematical Biosciences 1 (3), 327-374.

Frensing, T., Heldt, F. S., Pflugmacher, A., Behrendt, I., Jordan, I., Flockerzi, D., Genzel, Y., Reichl, U., 2013. Continuous influenza virus production in cell culture shows a periodic accumulation of defective interfering particles. PLOS ONE 8 (9), e72288.

Gavrieli, Y., Sherman, Y., Ben-Sasson, S. A., 1992. Identification of programmed cell death in situ via specific labeling of nuclear DNA fragmentation. Journal of Cell Biology 119 (3), 493-501.

Genzel, Y., Behrendt, I., König, S., Sann, H., Reichl, U., 2004. Metabolism of MDCK cells during cell growth and influenza virus production in large-scale microcarrier culture. Vaccine 22 (17-18), 2202-2208.

Green, D. R., 2005. Apoptotic pathways: ten minutes to dead. Cell 121 (5), 671-674.

Grosse, F., Manns, A., 1993. Terminal deoxyribonucleotidyl transferase (EC 2.7.7.31). In: Burrell, M. M. (Ed.), Enzymes of Molecular Biology. Vol. 16 of Methods in Molecular Biology. Humana Press, Totowa, New Jersey, USA, Ch. 7, pp. 95-105.

Halder, U. C., Bagchi, P., Chattopadhyay, S., Dutta, D., Chawla-Sarkar, M., 2011. Cell death regulation during influenza $A$ virus infection by matrix (M1) protein: a model of viral control over the cellular survival pathway. Cell Death \& Disease 2 (9), e197.

Haseltine, E. L., Rawlings, J. B., Yin, J., 2005. Dynamics of viral infections: incorporating both the intracellular and extracellular levels. Computers and Chemical Engineering 29 (3), 675-686.

Haseltine, E. L., Yin, J., Rawlings, J. B., Nov. 2008. Implications of decoupling the intracellular and extracellular levels in multi-level models of virus growth. Biotechnology and Bioengineering 101 (4), 811-820.

Hay, A. J., Gregory, V., Douglas, A. R., Lin, Y. P., Dec. 2001. The evolution of human influenza viruses. Philosophical Transactions of the Royal Society London B: Biological Sciences 356 (1416), 1861-1870.

Heynisch, B., Frensing, T., Heinze, K., Seitz, C., Genzel, Y., Reichl, U., 2010. Differen- 
tial activation of host cell signalling pathways through infection with two variants of influenza A/Puerto Rico/8/34 (H1N1) in MDCK cells. Vaccine 28 (51), 8210-8218.

Hierholzer, J. C., Killington, R. A., 1996. Virus isolation and quantitation. In: Mahy, B. W. J., Kangro, H. O. (Eds.), Virology Methods Manual. Academic Press, London, Ch. 2, pp. 25-46.

Hinshaw, V. S., Olsen, C. W., Dybdahl-Sissoko, N., Evans, D., 1994. Apoptosis: a mechanism of cell killing by influenza A and B viruses. Journal of Virology 68 (6), 3667-3673.

Holder, B. P., Beauchemin, C. A. A., 2011. Exploring the effect of biological delays in kinetic models of influenza within a host or cell culture. BMC Public Health 11 (Suppl 1), S10.

Katagiri, S., Ohizumi, A., Homma, M., Jul. 1983. An outbreak of type C influenza in a children's home. Journal of Infectious Diseases 148 (1), 51-56.

Kerr, J. F. R., Wyllie, A. H., Currie, A. R., 1972. Apoptosis: a basic biological phenomenon with wide-ranging implications in tissue kinetics. British Journal of Cancer 26 (4), 239-257.

Kirkwood, T. B., Bangham, C. R., 1994. Cycles, chaos, and evolution in virus cultures: a model of defective interfering particles. Proceedings of the National Academy of Sciences 91 (18), 8685-8689.

Kurokawa, M., Koyama, A. H., Yasuoka, S., Adachi, A., 1999. Influenza virus overcomes apoptosis by rapid multiplication. International Journal of Molecular Medicine 3 (5), $527-530$.

Lamb, R. A., Krug, R. M., 2001. Orthomyxoviridae: the viruses and their replication. In: Knipe, D. M., Howley, P. M. (Eds.), Fields virology, $4^{\text {th }}$ edition. Lippincott Williams \& Wilkins, pp. 1487-1531.

Licari, P., Bailey, J. E., 1992. Modeling the population dynamics of baculovirus-infected insect cells: optimizing infection strategies for enhanced recombinant protein yields. Biotechnology and Bioengineering 39 (4), 432-441.

Lindstrom, S. E., Hiromoto, Y., Nishimura, H., Saito, T., Nerome, R., Nerome, K., May 1999. Comparative analysis of evolutionary mechanisms of the hemagglutinin and three internal protein genes of influenza B virus: multiple cocirculating lineages and frequent reassortment of the NP, M, and NS genes. Journal of Virology 73 (5), 4413-4426. 
Liu, X., Li, P., Widlak, P., Zou, H., Luo, X., Garrard, W. T., Wang, X., 1998. The 40-kDa subunit of DNA fragmentation factor induces DNA fragmentation and chromatin condensation during apoptosis. Proceedings of the National Academy of Sciences 95 (15), $8461-8466$.

Liu, X., Zou, H., Slaughter, C., Wang, X., 1997. DFF, a heterodimeric protein that functions downstream of caspase-3 to trigger DNA fragmentation during apoptosis. Cell 89 (2), 175-184.

Lowy, R. J., 2003. Influenza virus induction of apoptosis by intrinsic and extrinsic mechanisms. International Reviews of Immunology 22 (5-6), 425-449.

Ludwig, S., Pleschka, S., Planz, O., Wolff, T., 2006. Ringing the alarm bells: signalling and apoptosis in influenza virus infected cells. Cellular Microbiology 8 (3), 375-386.

Luzyanina, T., Roose, D., Bocharov, G., Nov. 2009. Distributed parameter identification for a label-structured cell population dynamics model using CFSE histogram timeseries data. Journal of Mathematical Biology 59 (5), 581-603.

Mangold, M., Kienle, A., Gilles, E. D., Mohl, K. D., 2000. Nonlinear computation in DIVA - methods and applications. Chemical Engineering Science 55, 441-454.

Marcus, P. I., Ngunjiri, J. M., Sekellick, M. J., 2009. Dynamics of biologically active subpopulations of influenza virus: plaque-forming, noninfectious cell-killing, and defective interfering particles. Journal of Virology 83 (16), 8122-8130.

Matlab, 2012. Release R2012a. MathWorks, Natick, MA, USA.

Möhler, L., Flockerzi, D., Sann, H., Reichl, U., Apr. 2005. Mathematical model of influenza A virus production in large-scale microcarrier culture. Biotechnology and Bioengineering 90 (1), 46-58.

Müller, T., Dürr, R., Isken, B., Schulze-Horsel, J., Reichl, U., Kienle, A., 2011. Population balance modelling of influenza virus replication during vaccine production - Influence of apoptosis. In: Pistikopoulos, E., Georgiadis, M., Kokossis, A. (Eds.), $21^{\text {st }}$ European Symposium on Computer Aided Process Engineering - Part B. pp. 1336-1340.

Müller, T., Dürr, R., Isken, B., Schulze-Horsel, J., Reichl, U., Kienle, A., Aug. 2013. Distributed modeling of human influenza A virus-host cell interactions during vaccine production. Biotechnology and Bioengineering 110 (8), 2252-2266.

Müller, T., Schulze-Horsel, J., Sidorenko, Y., Reichl, U., Kienle, A., 2008. Population 
balance modeling of influenza virus replication in MDCK cells during vaccine production. In: Braunschweig, B., Joulia, X. (Eds.), $18^{\text {th }}$ European Symposium on Computer Aided Process Engineering. pp. 133-138.

Moeller, A., Kirchdoerfer, R. N., Potter, C. S., Carragher, B., Wilson, I. A., 2012. Organization of the influenza virus replication machinery. Science 338 (6114), 1631-1634.

Morris, S. J., Nightingale, K., Smith, H., Sweet, C., 2005. Influenza A virus-induced apoptosis is a multifactorial process: exploiting reverse genetics to elucidate the role of influenza A virus proteins in virus-induced apoptosis. Virology 335 (2), 198-211.

Nagata, S., 2000. Apoptotic DNA fragmentation. Experimental Cell Research 256 (1), $12-18$.

Nayak, D. P., Chambers, T. M., Akkina, R. K., 1989. Structure of defective-interfering RNAs of influenza viruses and their role in interference. In: Krug, R. M. (Ed.), The Influenza Viruses. Springer, pp. 269-317.

Nichols, J. E., LeDuc, J. W., 2009. Influenza. In: Barrett, A. D. T., Stanberry, L. R. (Eds.), Vaccines for biodefense and emerging and neglected Diseases. Academic Press, London, pp. 497-525.

Nichols, J. E., Mock, D. J., Roberts jr., N. J., 1993. Use of FITC-labeled influenza virus and flow cytometry to assess binding and internalization of virus by monocytesmacrophages and lymphocytes. Archives of Virology 130 (3-4), 441-455.

Nielsen, L. K., 2000. Virus production from cell culture, kinetics. In: Spier, R. E. (Ed.), Encyclopedia of Cell Technology. Vol. 2. John Wiley \& Sons, New York, pp. 1217-1230.

Nobusawa, E., Sato, K., Apr. 2006. Comparison of the mutation rates of human influenza A and B viruses. Journal of Virology 80 (7), 3675-3678.

Osterhaus, A. D. M. E., Rimmelzwaan, G. F., Martina, B. E. E., Bestebroer, T. M., Fouchier, R. A. M., May 2000. Influenza B virus in seals. Science 288 (5468), 10511053.

Power, J. F., Greenfield, P. F., Nielsen, L. K., Reid, S., 1992. Modelling the growth and protein production by insect cells following infection by a recombinant baculovirus in suspension culture. Cytotechnology 9 (1-3), 149-155.

Power, J. F., Nielsen, L. K., 1996. Modelling baculovirus infection of insect cells in culture. Cytotechnology 20 (1-3), 209-219. 
Power, J. F., Reid, S., Radford, K. M., Greenfield, P. F., Nielsen, L. K., 1994. Modeling and optimization of the baculovirus expression vector system in batch suspension culture. Biotechnology and Bioengineering 44 (6), 710-719.

Ramkrishna, D., 1979. Statistical models of cell populations. In: Advances in Biochemical Engineering. Vol. 11. Springer, Berlin, pp. 1-47.

Ramkrishna, D., 2000. Population Balances - Theory and Applications to Particulate Systems in Engineering. Academic Press, San Diego.

Ramkrishna, D., Mahoney, A. W., 2002. Population balance modeling. Promise for the future. Chemical Engineering Science 57 (4), 595-606.

Robert Koch Institut, Jan. 2011. Influenza - RKI-Ratgeber für Ärzte. http://www.rki . de/influenza.

Sakahira, H., Enari, M., Nagata, S., 1998. Cleavage of CAD inhibitor in CAD activation and DNA degradation during apoptosis. Nature 391 (6662), 96-99.

Schultz-Cherry, S., Dybdahl-Sissoko, N., Neumann, G., Kawaoka, Y., Hinshaw, V. S., 2001. Influenza virus NS1 protein induces apoptosis in cultured cells. Journal of Virology 75 (17), 7875-7881.

Schulze-Horsel, J., 2011. Zellphysiologische Charakterisierung von Zellkulturen in der Influenza-Impfstoffproduktion. No. 30 in Forschungsberichte aus dem Max-PlanckInstitut für Dynamik komplexer technischer Systeme. Shaker Verlag, Aachen, PhD thesis.

Schulze-Horsel, J., Genzel, Y., Reichl, U., 2008. Flow cytometric monitoring of influenza A virus infection in MDCK cells during vaccine production. BMC Biotechnology $8(45)$.

Schulze-Horsel, J., Schulze, M., Agalaridis, G., Genzel, Y., Reichl, U., 2009. Infection dynamics and virus-induced apoptosis in cell culture-based influenza vaccine production - Flow cytometry and mathematical modeling. Vaccine 27, 2712-2722.

Seitz, C., Frensing, T., Höper, D., Kochs, G., Reichl, U., 2010. High yields of influenza A virus in Madin-Darby canine kidney cells are promoted by an insufficient interferoninduced antiviral state. Journal of General Virology 91 (7), 1754-1763.

Sherer, E., Tocce, E., Hannemann, R. E., Rundell, A. E., Ramkrishna, D., Mar. 2008. 
Identification of age-structured models: cell cycle phase transitions. Biotechnology and Bioengineering 99 (4), 960-974.

Sidorenko, Y., Schulze-Horsel, J., Voigt, A., Reichl, U., Kienle, A., 2008a. Stochastic population balance modeling of influenza virus replication in vaccine production processes. Chemical Engineering Science 63, 157-169.

Sidorenko, Y., Voigt, A., Schulze-Horsel, J., Reichl, U., Kienle, A., 2008b. Stochastic population balance modeling of influenza virus replication in vaccine production processes. II. Detailed description of the replication mechanism. Chemical Engineering Science 63, 2299-2304.

Spencer, S. L., Gaudet, S., Albeck, J. G., Burke, J. M., Sorger, P. K., 2009. Non-genetic origins of cell-to-cell variability in TRAIL-induced apoptosis. Nature 459 (7245), 428 432 .

Srienc, F., May. 1999. Cytometric data as the basis for rigorous models of cell population dynamics. Journal of Biotechnology 71 (1-3), 233-238.

Takizawa, T., Matsukawa, S., Higuchi, Y., Nakamura, S., Nakanishi, Y., Fukuda, R., 1993. Induction of programmed cell death (apoptosis) by influenza virus infection in tissue culture cells. Journal of General Virology 74 (11), 2347-2355.

Taubenberger, J. K., Morens, D. M., Feb. 2008. The pathology of influenza virus infections. Annual Review of Pathology 3, 499-522.

Tran, A. T., Cortens, J. P., Du, Q., Wilkins, J. A., Coombs, K. M., 2013. Influenza virus induces apoptosis via BAD-mediated mitochondrial dysregulation. Journal of Virology 87 (2), 1049-1060.

Vester, D., Rapp, E., Kluge, S., Genzel, Y., Reichl, U., 2010. Virus-host cell interactions in vaccine production cell lines infected with different human influenza A virus variants: a proteomic approach. Journal of Proteomics 73 (9), 1656-1669.

Webster, R. G., Bean, W. J., Gorman, O. T., Chambers, T. M., Kawaoka, Y., Mar. 1992. Evolution and ecology of influenza A viruses. Microbiological Reviews 56 (1), $152-179$.

World Health Organization, Apr. 2009. Fact sheet - Influenza (Seasonal). http://www . who.int/mediacentre/factsheets/fs211/en/index.html.

Wurzer, W. J., Ehrhardt, C., Pleschka, S., Berberich-Siebelt, F., Wolff, T., Walczak, 
H., Planz, O., Ludwig, S., 2004. NF- $\kappa$ B-dependent induction of tumor necrosis factorrelated apoptosis-inducing ligand (TRAIL) and Fas/FasL is crucial for efficient influenza virus propagation. Journal of Biological Chemistry 279 (30), 30931-30937.

Wurzer, W. J., Planz, O., Ehrhardt, C., Giner, M., Silberzahn, T., Pleschka, S., Ludwig, S., 2003. Caspase 3 activation is essential for efficient influenza virus propagation. The EMBO Journal 22 (11), 2717-2728.

Zhirnov, O. P., Klenk, H.-D., 2007. Control of apoptosis in influenza virus-infected cells by up-regulation of Akt and p53 signaling. Apoptosis 12 (8), 1419-1432.

Zhirnov, O. P., Konakova, T. E., Garten, W., Klenk, H.-D., 1999. Caspase-dependent $\mathrm{N}$-terminal cleavage of influenza virus nucleocapsid protein in infected cells. Journal of Virology 73 (12), 10158-10163.

Zhirnov, O. P., Konakova, T. E., Wolff, T., Klenk, H.-D., 2002. NS1 protein of influenza A virus down-regulates apoptosis. Journal of Virology 76 (4), 1617-1625. 